\title{
SEARCHES FOR LORENTZ VIOLATION IN TOP-QUARK PRODUCTION AND DECAY AT HADRON COLLIDERS
}

\author{
Denver W. Whittington
}

Submitted to the faculty of the University Graduate School in partial fulfillment of the requirements

for the degree

Doctor of Philosophy

in the Department of Physics,

Indiana University

July 2012 
Accepted by the Graduate Faculty, Indiana University, in partial fulfillment of the requirements for the degree of Doctor of Philosophy.

Doctoral Committee

Harold G. Evans, Ph.D.

Sabine Lammers, Ph.D.

Frederick Luehring, Ph.D.

John M. Beggs, Ph.D.

July 6, 2012 
Copyright (c) 2012

Denver W. Whittington 
To my parents. 


\section{Acknowledgments}

The journey to reach this point has been long but rewarding. Physics has proven to be a challenging subject, and the experience has rewarded me with rich new perspectives on the world and its workings. I owe many thanks to a number of people who have provided valuable contributions to my growth as a physicist.

First and foremost among my colleagues, I wish to thank my thesis advisor Hal Evans. His contagious enthusiasm for particle physics propelled me through several periods of doldrums during my graduate studies. He has been an example to look toward time and again, both as a researcher and teacher. I look forward to my career in physics with confidence thanks in large part to his guidance.

Thanks also go to my colleagues at Indiana University. The other members of my thesis committee - Sabine Lammers, Fred Luehring, and John Beggs - have been enthusiastic about my analysis and have helped me produce a quality dissertation. My appreciation for the support of my colleagues at IU working on the ATLAS and DØ experiments cannot be understated. I have enjoyed their acquaintances both as professional collaborators and as friends. I could not have asked for a kinder or friendlier group with which to pursue my graduate work.

The analysis could not have been carried out without the work of several colleagues on the $\mathrm{D} \varnothing$ collaboration. Attaining these results has only been possible thanks to the various contributions from the other principal authors: Micheal Berger, Tom Ferbel, Hal Evans, Gaston Gutierrez, Andreas Jung, V. Alan Kostelecký, Michael Wang, and Zhenyu Ye. Particular thanks go to Gaston Gutierrez and Andreas Jung, who 
spearheaded the analysis effort when I first joined it. Of course, I also wish to express appreciation to the many members of the $\mathrm{D} \varnothing$ collaboration whose work to build and operate such a quality detector forms the backbone of every D $\varnothing$ analysis.

The long road of my physics education has been paved by a number of exceptional teachers, both during my time as a graduate student and before that as an undergraduate. Dale Bales, Don Cruikshank, Stan Stephens, and Darrel Austin were engaged and personable undergraduate professors, whose examples have positively informed my perspectives on teaching in academia. My research career got off to a good start thanks to the guidance of Adam Szczepaniak and Andy Bacher at Indiana University during my summer research experience. My graduate professors - Mike Snow, Sima Setayeshgar, Bill Schaich, Chuck Horowitz, John Beggs, and Steve Vigdor - all provided stimulating challenges to attack which built the foundations of my expertise in physics.

None of my accomplishments could have been achieved without the love and support of my family and friends. Numerous friends over the years - in Anderson, Bloomington, and at CERN - have shared the best times and been there for me in the worst times. In particular, my good friend Yi Yang has been a professional example in terms of graduate work and has provided a good deal of (mutual) support in the trying times of life as a graduate student. My brother, Skyler Whittington, has been a personal example of integrity and honor, and his accomplishments make me proud. My mother, Sue Whittington, has been an endless source of support and encouragement throughout my life. My late father, Chris Whittington, set an example through his generosity, humor, and boundless intellectual curiosity. Together my parents always encouraged me to follow my own curiosity, apply my talents, and seek 
to understand the world around me. I couldn't have made it here without them.

Finally, my fiancée Mary Camozzi has enriched my life and filled me with excitement for the future. We have had several adventures since she has joined me on my journey, and I look forward to many more. I owe a great deal of the quality of my physics communication skills to her valuable discussions and feedback, exemplified in the preface and first chapter of this dissertation. Her unique perspectives complement my own and help me understand and engage with the world in new and enjoyable ways. She is the most important part of my life, and with her in it I have more confidence in the future and in myself than I thought possible.

Thank you all. 


\section{Preface}

This document is the culmination of my graduate education in physics. It is a summary of the motivation, methods, and results of the analysis I conducted at experiments using the world's two largest particle accelerators. Out of necessity for efficient communication of the methods and results there will be judicious use of moderately advanced analytical and computational mathematics. However, for the casual reader (my family and friends), as well as for the introductory graduate student (whose daunting learning curve I remember well), I endeavor at every point to include a clear written description of each step. The introduction is intended to be useful for the graduate student beginning his or her career, while still remaining accessible to the non-scientist with an interest in particle physics and the research discussed herein.

\section{Why study physics?}

There seems to be a general perception that to study physics is to tackle one of the most difficult disciplines in academia. For this reason, many prospective young students seem to find themselves averted from the idea. It is true that the material is difficult, in large part because of the daunting mathematical skills required to arrive at formal results. Nonetheless, it is a worthwhile subject to pursue. Even an 
introduction to the fundamental concepts of physics bestows unique and valuable new perspectives. As Carl Sagan put it, "The cosmos is rich beyond measure - in elegant facts, in exquisite interrelationships, in the subtle machinery of awe." ${ }^{1}$ As one delves further into the intricacies of physics, more of this complex beauty is revealed.

When one understands how physical phenomena operate at the general level, they become suddenly obvious in a variety of often beautiful examples. The iridescent wings of a butterfly or the colors inside a sea shell are striking examples of how diffraction and interference contribute to the beauty in nature. Through application of the skills learned in an education in physics, such fundamental concepts can be harnessed in novel ways to make precision measurements which provide deep insights into the workings of nature. Each such basic physical principal can lead to discoveries across a wide variety of scales, ranging from the detection of planetary systems being born around other stars ${ }^{2}$ to observations of how matter behaves at the tiniest of sizes. ${ }^{3}$

To go beyond the concepts and rigorously pursue the depth that physics has to offer, a physicist requires a strong grasp of mathematics and an ability to translate abstract concepts into mathematical formulas. Such translation is vital for rapid and efficient communication and manipulation of ideas. The successful student of physics acquires an entirely new language of abstract representations. Despite the difficulties of translating phenomena between the observable and the mathematically abstract, any physicist will undoubtedly extol the virtues of the skills developed along this path of education.

A trained physicist has built a powerful set of skills and perspectives for solv-

${ }^{1}$ C. Sagan, Cosmos (Random House, New York, 1980).

${ }^{2}$ J.A. Eisner et al., Astrophys. J. 718, 774 (2010).

${ }^{3}$ K. Nakamura et al. (Particle Data Group), J. Phys. G 37, 075021 (2010). 
ing problems. Multiple variables, both observable and inferred, influence even the simplest of systems and must be considered. Complicating the matter, the variables in physical systems regularly influence each other in non-trivial ways. The resulting mathematical formulations are often impossible to solve exactly, requiring an expert ability to craft computer programs to find an approximate solution. The probabilistic (quantum) nature of the subatomic world and the statistical interpretation of experimental measurements together add yet another layer of complexity. Specialized mathematical techniques to account for unavoidable uncertainties in the results must be applied. Over the course of his or her career, the physicist sharpens the skills to recognize and tackle each of these facets in whatever form they appear.

It is the ability to apply these rigorous and logical techniques to novel problems that is the strongest advantage of an education in physics. The frontiers of science have moved to the study of complex interacting systems which blur the lines between traditionally separate fields and require novel new approaches and insights. The rise of biophysics as a popular discipline buzzing with activity illustrates just how powerful insights from physics can be when applied to biological systems. At the crossover between atomic physics, materials science, and chemistry, physicists are pioneering the blossoming fields of nanotechnologies. Those in possession of the skills developed through an advanced education in physics are integral to emerging fields of promising new interdisciplinary research.

There is also much to be gained by pushing the boundaries of knowledge and studying the advanced frontiers of pure physics. Researchers continue to probe deeper into big questions about the nature of the universe. Particle physicists are designing ever grander experiments in an effort to discover the fundamental constituents of 
matter. Cutting-edge accelerator facilities are producing energy densities consistent with the earliest moments after the big bang, seeking to find the origin of mass, extra dimensions of space, or new fundamental forces. This is an exciting time to be involved in physics research. 


\section{Denver W. Whittington}

\section{SEARCHES FOR LORENTZ VIOLATION IN TOP-QUARK PRODUCTION AND DECAY AT HADRON COLLIDERS}

We present a first-of-its-kind confirmation that the most massive known elementary particle obeys the special theory of relativity. Lorentz symmetry is a fundamental aspect of special relativity which posits that the laws of physics are invariant regardless of the orientation and velocity of the reference frame in which they are measured. Because this symmetry is a fundamental tenet of physics, it is important to test its validity in all processes. We quantify violation of this symmetry using the Standard-Model Extension framework, which predicts the effects that Lorentz violation would have on elementary particles and their interactions. The top quark is the most massive known elementary particle and has remained inaccessible to tests of Lorentz invariance until now. This model predicts a dependence of the production cross section for top and antitop quark pairs on sidereal time as the orientation of the experiment in which these events are produced changes with the rotation of the Earth. Using data collected with the D $\varnothing$ detector at the Fermilab Tevatron Collider, we search for violation of Lorentz invariance in events involving the production of a $t \bar{t}$ pair. Within the experimental precision, we find no evidence for such a violation and set upper limits on parameters describing its possible strength within the Standard 
Model Extension. We also investigate the prospects for extending this analysis using the ATLAS detector at the Large Hadron Collider which, because of the higher rate of $t \bar{t}$ events at that experiment, has the potential to improve the limits presented here. 


\section{Contents}

1 Introduction 1

1.1 Overview of the Standard Model . . . . . . . . . . . . . . . . 3

1.1.1 The Standard Model . . . . . . . . . . . . . . . . . 3

1.1.2 The top quark . . . . . . . . . . . . . . . . 11

1.2 Modern experimental collider physics . . . . . . . . . . . . . . . . 12

1.3 Symmetry .............................. 16

1.3.1 Lorentz symmetry . . . . . . . . . . . . . 18

1.3.2 CPT symmetry ...................... 21

2 The Standard-Model Extension 23

2.1 The Standard Model Lagrangian _. . . . . . . . . . . . . . . . . 24

2.2 SME addition to the top quark sector . . . . . . . . . . . . 29

2.3 Reference frames . . . . . . . . . . . . . . . . . . 30

2.4 Some previous SME studies . . . . . . . . . . . . . . . . . 32

3 Lorentz Violation in the Top-Quark Sector 35

3.1 SME matrix element . . . . . . . . . . . . . . . . . . . 37

3.2 Time-dependent cross section . . . . . . . . . . . . . . . 40 
3.2.1 Monte Carlo approximation . . . . . . . . . . . . . . 42

3.3 Experimental signature . . . . . . . . . . . . . . . . 46

4 The DØ Experiment $\quad 48$

4.1 The Fermilab Tevatron collider . . . . . . . . . . . . . . 50

4.2 The $\mathrm{D} \varnothing$ detector . . . . . . . . . . . . . . . . 53

4.2.1 Central tracking system . . . . . . . . . . . . . 54

4.2.2 Calorimetry ....................... 57

4.2 .3 Muon spectrometer ................. 58

4.2.4 Luminosity monitor . . . . . . . . . . . . . . . . . . 60

4.2.5 Trigger ..................... . . 60

5 Top Quark Event Selection at DØ 62

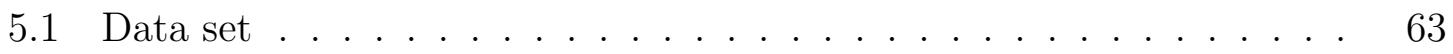

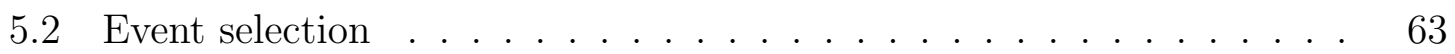

5.3 Sample composition . . . . . . . . . . . . . . . . . 66

5.4 Event times . . . . . . . . . . . . . . . . . . 70

6 Analysis at the DØ Experiment $\quad 72$

6.1 Kinematic terms . . . . . . . . . . . . . . 73

6.2 Reference frame transformation . . . . . . . . . . . . 75

6.3 Forms for $f_{\mathrm{SME}}(t) \ldots \ldots \ldots \ldots \ldots$. . . . . . . . . . . . 77

6.4 Expected event rate . . . . . . . . . . . . . . . . . . 82

6.5 Studies of Monte Carlo ensembles . . . . . . . . . . . . . 86

6.6 Amplitude of sidereal dependence . . . . . . . . . . . . . . . . 90

6.6.1 Electron amplitude results ................ . 91 
6.6.2 Muon amplitude results . . . . . . . . . . . . . . . . 92

6.7 Systematic uncertainties . . . . . . . . . . . . . . . . . 93

6.8 Cross-checks . . . . . . . . . . . . . . . . . . . . . . . 95

6.8.1 Background-enhanced samples . . . . . . . . . . . . . . . 96

6.8 .2 Binning effects $\ldots \ldots \ldots \ldots \ldots$

6.9 Measured SME coefficients . . . . . . . . . . . . . . . . . . . . . . 99

6.9 .1 Electron sample results . . . . . . . . . . . . . . . . 100

6.9 .2 Muon sample results . . . . . . . . . . . . . . . . . . 101

6.9.3 Simultaneous fit results . . . . . . . . . . . . . . . . . . 102

7 Prospective Analysis at the ATLAS Experiment 103

7.1 The ATLAS detector . . . . . . . . . . . . . . . . . . . 104

7.1.1 Large Hadron Collider . . . . . . . . . . . . . . . . . . 105

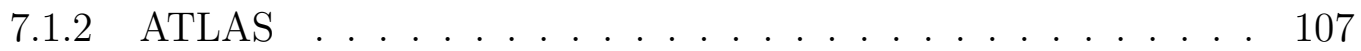

7.2 Expected signature . . . . . . . . . . . . . . . . . . . 111

7.2.1 Detector location and orientation . . . . . . . . . . . 111

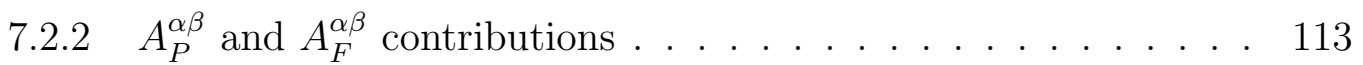

7.3 Event selection $\ldots \ldots \ldots \ldots \ldots \ldots \ldots \ldots \ldots$

7.4 Expected sensitivity _ . . . . . . . . . . . . . . . . . 119

7.4.1 Power spectrum of the event rate . . . . . . . . . . . 121

7.5 Likelihood analysis . . . . . . . . . . . . . . . . . . . . . . 124

$7.6 \quad$ Further possibilities . . . . . . . . . . . . . . . . . . . . 126

8 Conclusions 128

$\begin{array}{ll}\text { A List of Triggers for DØ Data } & 137\end{array}$ 
B Contributions to $A_{P}^{\alpha \beta}$ and $A_{F}^{\alpha \beta}$

C Systematics in MC Summation

C.1 Uncertainties for $e+$ jets . . . . . . . . . . . . . . . . 145

C.2 Uncertainties for $\mu+$ jets $\ldots \ldots \ldots \ldots \ldots$

$\begin{array}{ll}\text { D } \ell+n \text {-jets Cross-Checks } & 148\end{array}$

D.1 $\ell+2$-jets cross-check . . . . . . . . . . . . . . . . . 148

D.1.1 Electron amplitudes . . . . . . . . . . . . . . . . 149

D.1.2 Muon amplitudes . . . . . . . . . . . . . . . . . . 150

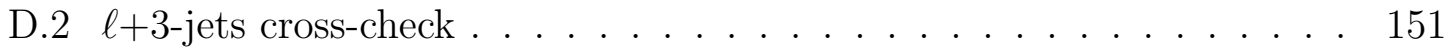

D.2.1 Electron amplitudes . . . . . . . . . . . . . . . 152

D.2.2 Muon amplitudes . . . . . . . . . . . . . . . . . 153

D.3 Agreement across samples . . . . . . . . . . . . . . . 154

E Fit Results: Run IIa and Run IIb 155

E.1 $\ell+2$-jets in Run IIa . . . . . . . . . . . . . . . . . . . 156

E.1.1 Electron amplitudes . . . . . . . . . . . . . . 157

E.1.2 Muon amplitudes . . . . . . . . . . . . . . . . . . 158

E.2 $\ell+3$-jets in Run IIa . . . . . . . . . . . . . . . . . . . . 159

E.2.1 Electron amplitudes . . . . . . . . . . . . . 160

E.2.2 Muon amplitudes . . . . . . . . . . . . . . . . . . . . 161

E.3 $\ell+>$-jets in Run IIa . . . . . . . . . . . . . . . . . . . . 162

E.3.1 Electron amplitudes . . . . . . . . . . . . . . 163

E.3.2 Muon amplitudes . . . . . . . . . . . . . . . . . . . . 164

E.4 $\ell+2$-jets in Run IIb . . . . . . . . . . . . . . . . . . . 165 
E.4.1 Electron amplitudes . . . . . . . . . . . . . . 166

E.4.2 Muon amplitudes . . . . . . . . . . . . . . . 167

E.5 $\ell+3$-jets in Run IIb . . . . . . . . . . . . . . . . . . . . . . . . 168

E.5.1 Electron amplitudes . . . . . . . . . . . . . . . . 169

E.5.2 Muon amplitudes . . . . . . . . . . . . . . . . . . 170

E.6 $\ell+>3$-jets in Run IIb . . . . . . . . . . . . . . . . . . . . . . . 171

E.6.1 Electron amplitudes . . . . . . . . . . . . . . 172

E.6.2 Muon amplitudes ................. . 173

E.7 Agreement across samples . . . . . . . . . . . . . . . . . . . 174

$\begin{array}{lll}\text { F Data and Fits } & 175\end{array}$

F.1 $e+>3$-jets . . . . . . . . . . . . . . 176

F.2 $\mu+>3$-jets . . . . . . . . . . . . . . . 180

F.3 Simultaneous $e+>3$-jets and $\mu+>$-jets . . . . . . . . . . . . . 184 


\section{List of Tables}

1.1 Particles of the Standard Model. . . . . . . . . . . . . . . . . 8

5.1 Number of $e+$ jets $t \bar{t}$ candidates in the "tight" lepton selection with exactly $1 b$-tagged jet. . . . . . . . . . . . . 66

5.2 Number of $\mu+$ jets $t \bar{t}$ candidates in the "tight" lepton selection with exactly 1 b-tagged jet. . . . . . . . . . . . . . . . 66

5.3 Comparison of yields for $e+n$-jets channels for data in the $1 b$-tag selection to contributions expected from $t \bar{t}$ signal and sources of background. Uncertainties include statistical and systematic contributions. . . . . 68

5.4 Comparison of yields for $\mu+n$-jets channels for data in the $1 b$-tag selection to contributions expected from $t \bar{t}$ signal and sources of background. Uncertainties include statistical and systematic contributions.

6.1 Parameters defining the orientation of the $\mathrm{D} \varnothing$ detector . . . . . . 77

$6.2 f_{\mathrm{SME}}(t)$ under different SME assumptions. . . . . . . . . . . . . 78

6.3 Amplitude extracted from $e+>3$-jets $t \bar{t}$ candidates, with phase fixed appropriate to each coefficient case and assuming $\left(c_{R}\right)_{\mu \nu}=0 \ldots \ldots 91$ 
6.4 Amplitude extracted from $e+>3$-jets $t \bar{t}$ candidates, with phase fixed appropriate to each coefficient case and assuming $\left(c_{L}\right)_{\mu \nu}=0$. . . .

6.5 Amplitude extracted from $e+>3$-jets $t \bar{t}$ candidates, with phase fixed appropriate to each coefficient case and assuming $d_{\mu \nu}=0 . \quad$. . . . . 91

6.6 Amplitude extracted from $e+>3$-jets $t \bar{t}$ candidates, with phase fixed appropriate to each coefficient case and assuming $c_{\mu \nu}=0 \ldots \ldots$

6.7 Amplitude extracted from $\mu+>3$-jets $t \bar{t}$ candidates, with phase fixed appropriate to each coefficient case and assuming $\left(c_{R}\right)_{\mu \nu}=0$. . . .

6.8 Amplitude extracted from $\mu+>3$-jets $t \bar{t}$ candidates, with phase fixed appropriate to each coefficient case and assuming $\left(c_{L}\right)_{\mu \nu}=0 .$. . .

6.9 Amplitude extracted from $\mu+>3$-jets $t \bar{t}$ candidates, with phase fixed appropriate to each coefficient case and assuming $d_{\mu \nu}=0$. . . . .

6.10 Amplitude extracted from $\mu+>3$-jets $t \bar{t}$ candidates, with phase fixed appropriate to each coefficient case and assuming $c_{\mu \nu}=0 \ldots \ldots$

6.11 Signal fractions $f_{S}$ for $\ell+>3$-jets $t \bar{t}$ candidates. . . . . . . . . . . 93

6.12 A-matrix elements for $e+$ jets . . . . . . . . . . . . . . 94

$6.13 A$-matrix elements for $\mu+$ jets . . . . . . . . . . . . . . 95

$6.14 t \bar{t}$ fractions for the $\ell+n$-jets samples, $n=2,3 \ldots \ldots$. . . . . . 96

$6.15\left(c_{L}\right)_{\mu \nu}$ extracted from $e+$ jets $t \bar{t}$ candidates, assuming $\left(c_{R}\right)_{\mu \nu}=0 . \ldots 100$

$6.16\left(c_{R}\right)_{\mu \nu}$ extracted from $e+$ jets $t \bar{t}$ candidates, assuming $\left(c_{L}\right)_{\mu \nu}=0 . \ldots 100$

$6.17 c_{\mu \nu}$ extracted from $e+$ jets $t \bar{t}$ candidates, assuming $d_{\mu \nu}=0 . \ldots . . .100$

$6.18 d_{\mu \nu}$ extracted from $e+$ jets $t \bar{t}$ candidates, assuming $c_{\mu \nu}=0 . \quad \ldots . . \quad 100$

$6.19\left(c_{L}\right)_{\mu \nu}$ extracted from $\mu+$ jets $t \bar{t}$ candidates, assuming $\left(c_{R}\right)_{\mu \nu}=0 \ldots . \quad 101$

$6.20\left(c_{R}\right)_{\mu \nu}$ extracted from $\mu+$ jets $t \bar{t}$ candidates, assuming $\left(c_{L}\right)_{\mu \nu}=0 \ldots . .101$ 
$6.21 c_{\mu \nu}$ extracted from $\mu+$ jets $t \bar{t}$ candidates, assuming $d_{\mu \nu}=0 \ldots \ldots \ldots 101$

$6.22 d_{\mu \nu}$ extracted from $\mu+$ jets $t \bar{t}$ candidates, assuming $c_{\mu \nu}=0 \ldots \ldots \ldots 101$

$6.23\left(c_{L}\right)_{\mu \nu}$ extracted from simultaneous fits to $e+$ jets and $\mu+$ jets $t \bar{t}$ candidates, assuming $\left(c_{R}\right)_{\mu \nu}=0 . \ldots \ldots \ldots \ldots \ldots \ldots \ldots \ldots \ldots \ldots$

$6.24\left(c_{R}\right)_{\mu \nu}$ extracted from simultaneous fits to $e+$ jets and $\mu+$ jets $t \bar{t}$ candi-

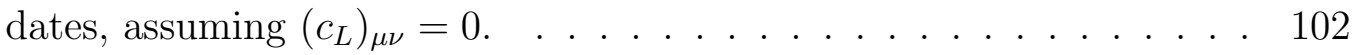

$6.25 c_{\mu \nu}$ extracted from simultaneous fits to $e+$ jets and $\mu+$ jets $t \bar{t}$ candidates, assuming $d_{\mu \nu}=0 \ldots \ldots \ldots \ldots \ldots \ldots \ldots \ldots$

$6.26 d_{\mu \nu}$ extracted from simultaneous fits to $e+$ jets and $\mu+$ jets $t \bar{t}$ candidates, assuming $c_{\mu \nu}=0 \ldots \ldots \ldots \ldots \ldots \ldots \ldots \ldots \ldots \ldots$

7.1 Comparison of yields for the $e+$ jets and $\mu+$ jets channels for the $t \bar{t}$ resonance study to contributions expected from $t \bar{t}$ signal and sources of background. The uncertainties given are due to systematic effects, while statistical uncertainties are small and neglected. . . . . . . . . 118

7.2 Comparison of signal sample size for the $e+$ jets and $\mu+$ jets channels for the $t \bar{t}$ resonance study to those used for the DØ analysis. . . . . . 119

7.3 The expected statistical uncertainty on $\left(c_{L}\right)_{\mu \nu}$ at ATLAS compared to that of the $\mathrm{D} \emptyset$ analysis. . . . . . . . . . . . . . . . . 120

7.4 The expected statistical uncertainty on $\left(c_{R}\right)_{\mu \nu}$ at ATLAS compared to that of the $\mathrm{D} \emptyset$ analysis. . . . . . . . . . . . . . . . . 120

7.5 The expected statistical uncertainty on $c_{\mu \nu}$ at ATLAS compared to that of the $\mathrm{D} \varnothing$ analysis. . . . . . . . . . . . . . . 120

7.6 The expected statistical uncertainty on $d_{\mu \nu}$ at ATLAS compared to that of the $\mathrm{D} \varnothing$ analysis. . . . . . . . . . . . . . . . . . . . 120 
8.1 Limits on SME coefficients at the 95\% C.L., assuming $\left(c_{U}\right)_{\mu \nu} \equiv 0$. . 130

8.2 Limits on SME coefficients at the $95 \%$ C.L., assuming $\left(c_{Q}\right)_{\mu \nu} \equiv 0$. . . 130

8.3 Limits on SME coefficients at the $95 \%$ C.L., assuming $c_{\mu \nu} \equiv 0$. . . . 130

A.1 Trigger and corresponding integrated luminosity analyzed for the $e+$ jets sample in Run IIa. . . . . . . . . . . . . . . . . . . . . . . . . . . . 137

A.2 Trigger and corresponding integrated luminosity analyzed for the $\mu+$ jets sample in Run IIa. . . . . . . . . . . . . . . . . . . . . . . . . 138

A.3 Trigger and corresponding integrated luminosity analyzed for the $e+$ jets and $\mu+$ jets samples in Run IIb. . . . . . . . . . . . . . . . . . 138

C.1 Uncertainties on $A_{P}^{t t} \quad \ldots \ldots \ldots \ldots$. . . . . . . . . . . 145

C.2 Uncertainties on $A_{F}^{t t} \quad \ldots \ldots \ldots \ldots$. . . . . . . . . . 145

C.3 Uncertainties on $A_{P}^{x x} \ldots \ldots \ldots \ldots$. . . . . . . . . . . . . . . . . . . 145

C.4 Uncertainties on $A_{F}^{x x} \ldots \ldots \ldots \ldots \ldots$. . . . . . . . . . . 145

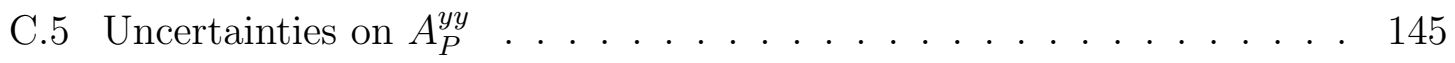

C.6 Uncertainties on $A_{F}^{y y} \ldots \ldots \ldots \ldots \ldots$. . . . . . . . . . 145

C.7 Uncertainties on $A_{P}^{z z} \ldots \ldots \ldots \ldots \ldots$. . . . . . . . . . 146

C.8 Uncertainties on $A_{F}^{z z} \ldots \ldots \ldots \ldots$

C.9 Uncertainties on $A_{P}^{t t} \quad \ldots \ldots \ldots \ldots$. . . . . . . . . . 146

C.10 Uncertainties on $A_{F}^{t t} \ldots \ldots \ldots \ldots \ldots$. . . . . . . . . . 146

C.11 Uncertainties on $A_{P}^{x x} \ldots \ldots \ldots \ldots$. . . . . . . . . . 146

C.12 Uncertainties on $A_{F}^{x x} \ldots \ldots \ldots \ldots \ldots$

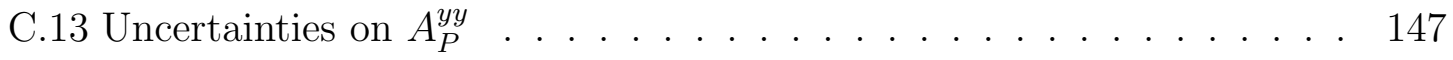

C.14 Uncertainties on $A_{F}^{y y} \ldots \ldots \ldots \ldots$. . . . . . . . . . 147 
C.15 Uncertainties on $A_{P}^{z z} \ldots \ldots \ldots \ldots \ldots \ldots$

C.16 Uncertainties on $A_{F}^{z z} \ldots \ldots \ldots \ldots \ldots \ldots$

D.1 Amplitude extracted from $e+2$-jets $t \bar{t}$ candidates, with phase fixed appropriate to each coefficient case and assuming $\left(c_{R}\right)_{\mu \nu}=0 . \ldots \ldots 149$

D.2 Amplitude extracted from $e+2$-jets $t \bar{t}$ candidates, with phase fixed appropriate to each coefficient case and assuming $\left(c_{L}\right)_{\mu \nu}=0 . \ldots \ldots 149$

D.3 Amplitude extracted from $e+2$-jets $t \bar{t}$ candidates, with phase fixed appropriate to each coefficient case and assuming $d_{\mu \nu}=0 \ldots \ldots \ldots$

D.4 Amplitude extracted from $e+2$-jets $t \bar{t}$ candidates, with phase fixed appropriate to each coefficient case and assuming $c_{\mu \nu}=0 \ldots \ldots \ldots 149$

D.5 Amplitude extracted from $\mu+2$-jets $t \bar{t}$ candidates, with phase fixed appropriate to each coefficient case and assuming $\left(c_{R}\right)_{\mu \nu}=0 \ldots \ldots 150$

D.6 Amplitude extracted from $\mu+2$-jets $t \bar{t}$ candidates, with phase fixed appropriate to each coefficient case and assuming $\left(c_{L}\right)_{\mu \nu}=0 \ldots \ldots 150$

D.7 Amplitude extracted from $\mu+2$-jets $t \bar{t}$ candidates, with phase fixed appropriate to each coefficient case and assuming $d_{\mu \nu}=0 . \ldots \ldots 150$

D.8 Amplitude extracted from $\mu+2$-jets $t \bar{t}$ candidates, with phase fixed appropriate to each coefficient case and assuming $c_{\mu \nu}=0 \ldots \ldots \ldots$

D.9 Amplitude extracted from $e+3$-jets $t \bar{t}$ candidates, with phase fixed appropriate to each coefficient case and assuming $\left(c_{R}\right)_{\mu \nu}=0 \ldots \ldots 152$

D.10 Amplitude extracted from $e+3$-jets $t \bar{t}$ candidates, with phase fixed appropriate to each coefficient case and assuming $\left(c_{L}\right)_{\mu \nu}=0 \ldots \ldots 152$

D.11 Amplitude extracted from $e+3$-jets $t \bar{t}$ candidates, with phase fixed appropriate to each coefficient case and assuming $d_{\mu \nu}=0 \ldots \ldots$. 
D.12 Amplitude extracted from $e+3$-jets $t \bar{t}$ candidates, with phase fixed appropriate to each coefficient case and assuming $c_{\mu \nu}=0 \ldots \ldots \ldots 152$

D.13 Amplitude extracted from $\mu+3$-jets $t \bar{t}$ candidates, with phase fixed appropriate to each coefficient case and assuming $\left(c_{R}\right)_{\mu \nu}=0 \ldots \ldots 153$

D.14 Amplitude extracted from $\mu+3$-jets $t \bar{t}$ candidates, with phase fixed appropriate to each coefficient case and assuming $\left(c_{L}\right)_{\mu \nu}=0 \ldots \ldots 153$

D.15 Amplitude extracted from $\mu+3$-jets $t \bar{t}$ candidates, with phase fixed appropriate to each coefficient case and assuming $d_{\mu \nu}=0 . \ldots \ldots 153$

D.16 Amplitude extracted from $\mu+3$-jets $t \bar{t}$ candidates, with phase fixed appropriate to each coefficient case and assuming $c_{\mu \nu}=0 \ldots \ldots 153$

D.17 The values of $\chi^{2}$ and probability reflecting consistency with no time dependence for the 4 independent measurements of amplitudes of sidereal oscillations in the cross-check channels $(\ell+n$-jets, where $\ell=e, \mu$ and $n=2,3)$ for each of the 16 phases assumed in this analysis. . . 154

E.1 Amplitude extracted from $e+2$-jets $t \bar{t}$ candidates, with phase fixed appropriate to each coefficient case and assuming $\left(c_{R}\right)_{\mu \nu}=0 \ldots \ldots 157$

E.2 Amplitude extracted from $e+2$-jets $t \bar{t}$ candidates, with phase fixed appropriate to each coefficient case and assuming $\left(c_{L}\right)_{\mu \nu}=0 \ldots \ldots 157$

E.3 Amplitude extracted from $e+2$-jets $t \bar{t}$ candidates, with phase fixed appropriate to each coefficient case and assuming $d_{\mu \nu}=0 \ldots \ldots \ldots 157$

E.4 Amplitude extracted from $e+2$-jets $t \bar{t}$ candidates, with phase fixed appropriate to each coefficient case and assuming $c_{\mu \nu}=0 \ldots \ldots \ldots$

E.5 Amplitude extracted from $\mu+2$-jets $t \bar{t}$ candidates, with phase fixed appropriate to each coefficient case and assuming $\left(c_{R}\right)_{\mu \nu}=0 \ldots \ldots 158$ 
E.6 Amplitude extracted from $\mu+2$-jets $t \bar{t}$ candidates, with phase fixed appropriate to each coefficient case and assuming $\left(c_{L}\right)_{\mu \nu}=0 . . . . \quad 158$

E.7 Amplitude extracted from $\mu+2$-jets $t \bar{t}$ candidates, with phase fixed appropriate to each coefficient case and assuming $d_{\mu \nu}=0 . \quad \ldots . . \quad 158$

E.8 Amplitude extracted from $\mu+2$-jets $t \bar{t}$ candidates, with phase fixed appropriate to each coefficient case and assuming $c_{\mu \nu}=0 \ldots \ldots$. . . 158

E.9 Amplitude extracted from $e+3$-jets $t \bar{t}$ candidates, with phase fixed appropriate to each coefficient case and assuming $\left(c_{R}\right)_{\mu \nu}=0 . \ldots 160$

E.10 Amplitude extracted from $e+3$-jets $t \bar{t}$ candidates, with phase fixed appropriate to each coefficient case and assuming $\left(c_{L}\right)_{\mu \nu}=0 . \quad \ldots . . \quad 160$

E.11 Amplitude extracted from $e+3$-jets $t \bar{t}$ candidates, with phase fixed appropriate to each coefficient case and assuming $d_{\mu \nu}=0 \ldots$. . . . . 160

E.12 Amplitude extracted from $e+3$-jets $t \bar{t}$ candidates, with phase fixed appropriate to each coefficient case and assuming $c_{\mu \nu}=0 . \ldots$. . . . . 160

E.13 Amplitude extracted from $\mu+3$-jets $t \bar{t}$ candidates, with phase fixed appropriate to each coefficient case and assuming $\left(c_{R}\right)_{\mu \nu}=0 . . . . \quad 161$

E.14 Amplitude extracted from $\mu+3$-jets $t \bar{t}$ candidates, with phase fixed appropriate to each coefficient case and assuming $\left(c_{L}\right)_{\mu \nu}=0 . \ldots . .161$

E.15 Amplitude extracted from $\mu+3$-jets $t \bar{t}$ candidates, with phase fixed appropriate to each coefficient case and assuming $d_{\mu \nu}=0 . \quad$. . . . 161

E.16 Amplitude extracted from $\mu+3$-jets $t \bar{t}$ candidates, with phase fixed appropriate to each coefficient case and assuming $c_{\mu \nu}=0 \ldots \ldots$. . . 161

E.17 Amplitude extracted from $e+>3$-jets $t \bar{t}$ candidates, with phase fixed appropriate to each coefficient case and assuming $\left(c_{R}\right)_{\mu \nu}=0 \ldots \ldots 163$ 
E.18 Amplitude extracted from $e+>3$-jets $t \bar{t}$ candidates, with phase fixed appropriate to each coefficient case and assuming $\left(c_{L}\right)_{\mu \nu}=0 . \ldots 163$

E.19 Amplitude extracted from $e+>3$-jets $t \bar{t}$ candidates, with phase fixed appropriate to each coefficient case and assuming $d_{\mu \nu}=0 . \quad \ldots$. . . 163

E.20 Amplitude extracted from $e+>3$-jets $t \bar{t}$ candidates, with phase fixed appropriate to each coefficient case and assuming $c_{\mu \nu}=0 \ldots \ldots$

E.21 Amplitude extracted from $\mu+>3$-jets $t \bar{t}$ candidates, with phase fixed appropriate to each coefficient case and assuming $\left(c_{R}\right)_{\mu \nu}=0 \ldots \ldots$

E.22 Amplitude extracted from $\mu+>3$-jets $t \bar{t}$ candidates, with phase fixed appropriate to each coefficient case and assuming $\left(c_{L}\right)_{\mu \nu}=0 . \ldots . .164$

E.23 Amplitude extracted from $\mu+>3$-jets $t \bar{t}$ candidates, with phase fixed appropriate to each coefficient case and assuming $d_{\mu \nu}=0 . \quad \ldots . . \quad 164$

E.24 Amplitude extracted from $\mu+>3$-jets $t \bar{t}$ candidates, with phase fixed appropriate to each coefficient case and assuming $c_{\mu \nu}=0 \ldots \ldots$

E.25 Amplitude extracted from $e+2$-jets $t \bar{t}$ candidates, with phase fixed appropriate to each coefficient case and assuming $\left(c_{R}\right)_{\mu \nu}=0 . \ldots 166$

E.26 Amplitude extracted from $e+2$-jets $t \bar{t}$ candidates, with phase fixed appropriate to each coefficient case and assuming $\left(c_{L}\right)_{\mu \nu}=0 . \ldots 166$

E.27 Amplitude extracted from $e+2$-jets $t \bar{t}$ candidates, with phase fixed appropriate to each coefficient case and assuming $d_{\mu \nu}=0 \ldots \ldots$. . . . 166

E.28 Amplitude extracted from $e+2$-jets $t \bar{t}$ candidates, with phase fixed appropriate to each coefficient case and assuming $c_{\mu \nu}=0 . \ldots 166$

E.29 Amplitude extracted from $\mu+2$-jets $t \bar{t}$ candidates, with phase fixed appropriate to each coefficient case and assuming $\left(c_{R}\right)_{\mu \nu}=0 \ldots \ldots 7$ 
E.30 Amplitude extracted from $\mu+2$-jets $t \bar{t}$ candidates, with phase fixed appropriate to each coefficient case and assuming $\left(c_{L}\right)_{\mu \nu}=0 . \ldots 167$

E.31 Amplitude extracted from $\mu+2$-jets $t \bar{t}$ candidates, with phase fixed appropriate to each coefficient case and assuming $d_{\mu \nu}=0 . \quad \ldots$. . . 167

E.32 Amplitude extracted from $\mu+2$-jets $t \bar{t}$ candidates, with phase fixed appropriate to each coefficient case and assuming $c_{\mu \nu}=0 \ldots \ldots$. . . 167

E.33 Amplitude extracted from $e+3$-jets $t \bar{t}$ candidates, with phase fixed appropriate to each coefficient case and assuming $\left(c_{R}\right)_{\mu \nu}=0 . \ldots$. . .

E.34 Amplitude extracted from $e+3$-jets $t \bar{t}$ candidates, with phase fixed appropriate to each coefficient case and assuming $\left(c_{L}\right)_{\mu \nu}=0 . \quad \ldots . . \quad 169$

E.35 Amplitude extracted from $e+3$-jets $t \bar{t}$ candidates, with phase fixed appropriate to each coefficient case and assuming $d_{\mu \nu}=0 \ldots$. . . . . 169

E.36 Amplitude extracted from $e+3$-jets $t \bar{t}$ candidates, with phase fixed appropriate to each coefficient case and assuming $c_{\mu \nu}=0 \ldots \ldots$. . .

E.37 Amplitude extracted from $\mu+3$-jets $t \bar{t}$ candidates, with phase fixed appropriate to each coefficient case and assuming $\left(c_{R}\right)_{\mu \nu}=0 \ldots \ldots$. . 170

E.38 Amplitude extracted from $\mu+3$-jets $t \bar{t}$ candidates, with phase fixed appropriate to each coefficient case and assuming $\left(c_{L}\right)_{\mu \nu}=0 . \ldots . \quad 170$

E.39 Amplitude extracted from $\mu+3$-jets $t \bar{t}$ candidates, with phase fixed appropriate to each coefficient case and assuming $d_{\mu \nu}=0 . \quad \ldots$. . . 170

E.40 Amplitude extracted from $\mu+3$-jets $t \bar{t}$ candidates, with phase fixed appropriate to each coefficient case and assuming $c_{\mu \nu}=0 \ldots \ldots$. . . 170

E.41 Amplitude extracted from $e+>3$-jets $t \bar{t}$ candidates, with phase fixed appropriate to each coefficient case and assuming $\left(c_{R}\right)_{\mu \nu}=0 \ldots \ldots$. . 172 
E.42 Amplitude extracted from $e+>3$-jets $t \bar{t}$ candidates, with phase fixed appropriate to each coefficient case and assuming $\left(c_{L}\right)_{\mu \nu}=0 \ldots \ldots 172$

E.43 Amplitude extracted from $e+>3$-jets $t \bar{t}$ candidates, with phase fixed appropriate to each coefficient case and assuming $d_{\mu \nu}=0 . \quad \ldots \ldots$.

E.44 Amplitude extracted from $e+>3$-jets $t \bar{t}$ candidates, with phase fixed appropriate to each coefficient case and assuming $c_{\mu \nu}=0 \ldots \ldots 172$

E.45 Amplitude extracted from $\mu+>3$-jets $t \bar{t}$ candidates, with phase fixed appropriate to each coefficient case and assuming $\left(c_{R}\right)_{\mu \nu}=0 \ldots \ldots$

E.46 Amplitude extracted from $\mu+>3$-jets $t \bar{t}$ candidates, with phase fixed appropriate to each coefficient case and assuming $\left(c_{L}\right)_{\mu \nu}=0 \ldots \ldots 173$

E.47 Amplitude extracted from $\mu+>3$-jets $t \bar{t}$ candidates, with phase fixed appropriate to each coefficient case and assuming $d_{\mu \nu}=0 . \ldots \ldots 173$

E.48 Amplitude extracted from $\mu+>3$-jets $t \bar{t}$ candidates, with phase fixed appropriate to each coefficient case and assuming $c_{\mu \nu}=0 \ldots \ldots \ldots$

E.49 The values of $\chi^{2}$ and probability reflecting consistency with no time dependence for the 12 independent measurements of amplitudes of sidereal oscillations in all channels $(\ell+n$-jets, where $\ell=e, \mu$ and $n=2,3, \geq$ 4 divided into Run IIa and Run IIb) for each of the 16 phases assumed

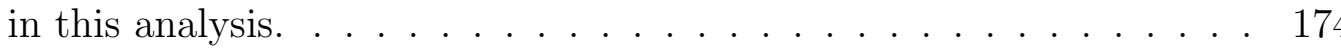




\section{List of Figures}

1.1 Zooming in on the structure of matter. (c) $1992 \mathrm{CERN}$ ) . . . . . . 4

1.2 Various subdetectors of the ATLAS detector and the particles they specialize in measuring. (ATLAS Experiment (c) 2012 CERN) . . . . 15

2.1 The canonical Sun-centered inertial reference frame of the SME. . . . 31

3.1 Illustration of the production and decay of a $t \bar{t}$ pair. . . . . . . . . . 36

3.2 Illustration of the relationship between the reference frame of an experiment and the Sun-centered reference frame. (Earth and Sun image credits: NASA) . . . . . . . . . . . . . . . . . . . . 47

4.1 The Fermilab accelerator complex. . . . . . . . . . . . . . 51

4.2 The DØ detector. A pair of physicists in their natural environment illustrates scale. . . . . . . . . . . . . . . . . . 53

4.3 Cross section of the DØ detector in $x-z \ldots \ldots . \ldots 54$

4.4 The DØ central tracking system. . . . . . . . . . . . . 55

4.5 The $\mathrm{D} \varnothing$ calorimeters. . . . . . . . . . . . . . . . . . 57

4.6 Exploded view of the PDT and MDT detectors. . . . . . . . . . . . 59

4.7 Exploded view of the scintillation trigger counters. . . . . . . . . . . 59 
4.8 The $\mathrm{D} \varnothing$ luminosity monitor. . . . . . . . . . . . . . . . . 60

4.9 Overview of the $\mathrm{D} \varnothing$ trigger and data acquisition systems. . . . . . . 61

5.1 Jet multiplicity distribution for $\ell+$ jets events with $1 b$-tagged jet for backgrounds and contributions from $t \bar{t}$ signal $(e+$ jets and $\mu+$ jets combined) $[36] . \ldots \ldots \ldots \ldots \ldots$

5.2 The number of $t \bar{t}$ candidates versus sidereal phase for (a) $e+>3$-jets events and (b) $\mu+>3$-jets events. The uncertainties reflect the sample size. . . . . . . . . . . . . . . . .

6.1 The relative luminosity $\mathcal{L}_{i} / \mathcal{L}_{\text {int }}$ by sidereal phase corresponding to (a) the $e+$ jets event selection and (b) the $\mu+$ jets event selection. . . . .

6.2 The dependence of $R$ on sidereal phase for (a) $e+>3$-jets $t \bar{t}$ candidates and (b) the $\mu+>3$-jets $t \bar{t}$ candidates. . . . . . . . . . . . .

6.3 Parameters extracted from studies of 10,000 MC pseudo-experiments, each containing 401 events with an input value of $\left(c_{L}\right)_{X X}=0.4$ : (a) values of $\left(c_{L}\right)_{X X}$, (b) the pull $=\left(\left(c_{L}\right)_{X X}-0.4\right) / \mathrm{sd}$, and (c) the probability of the $\chi^{2}$ of the fit for each pseudo-experiment. . . . . . . .

6.4 Dependence of (a) the mean extracted value of $\left(c_{L}\right)_{X X}$, (b) the mean of the pull distribution, and (c) the RMS of the pull distribution on the input value of $\left(c_{L}\right)_{X X}$ for different values of sidereal binning: 12 bins (black), 24 bins (red), and 36 bins (blue). . . . . . . . .

7.1 The ATLAS detector and its major subsystems. Several technicians next to the detector give a sense of the immense scale of the apparatus. (ATLAS Experiment (C) 2012 CERN) . . . . . . . . . . . 105 
7.2 The accelerator complex at CERN, including the stages leading to the LHC. Various accelerators are labeled, along with their size and the year they were installed. The figure is not to scale. (c) $2008 \mathrm{CERN}$ )

7.3 Summary of measurements of the $t \bar{t}$ production cross section compared to the corresponding theoretical expectation. The upper part of the figure shows measurements that are averaged to give the combined value shown. The lower part shows additional newer measurements not included in the combination. (ATLAS Experiment (c) 2012 CERN) 116

7.4 The Periodogram used by the BaBar experiment to search for signs of CPT and Lorentz violation through variations in $B^{0}-\bar{B}^{0}$ oscillations observed through opposite-sign dilepton events in $\Upsilon(4 S) \rightarrow B \bar{B}$ decays [20]. The triangles in the inset indicate the solar and sidereal frequencies. No significant signal appears at any frequency. . . . . . . 123

B.1 Distribution of event-by-event contributions to the elements of $A_{P}^{\alpha \beta}$ in the simulated $e+$ jets sample. Rows and columns are ordered $(t, x, y, z) .140$

B.2 Distribution of event-by-event contributions to the elements of $A_{F}^{\alpha \beta}$ in the simulated $e+$ jets sample. Rows and columns are ordered $(t, x, y, z) .141$

B.3 Distribution of event-by-event contributions to the elements of $A_{P}^{\alpha \beta}$ in the simulated $\mu+$ jets sample. Rows and columns are ordered $(t, x, y, z) .142$

B.4 Distribution of event-by-event contributions to the elements of $A_{F}^{\alpha \beta}$ in the simulated $\mu+$ jets sample. Rows and columns are ordered $(t, x, y, z) .143$

D.1 Number of $\ell+2$-jets $t \bar{t}$ candidates per two sidereal hours. . . . . . . . 148

D.2 Number of $\ell+3$-jets $t \bar{t}$ candidates per two sidereal hours. . . . . . . . 151 
E.1 Number of $\ell+2$-jets $t \bar{t}$ candidates per two sidereal hours, Run IIa. . . 156

E.2 Number of $\ell+3$-jets $t \bar{t}$ candidates per two sidereal hours, Run IIa. . . 159

E.3 Number of $\ell+>3$-jets $t \bar{t}$ candidates per two sidereal hours, Run IIa. . 162

E.4 Number of $\ell+2$-jets $t \bar{t}$ candidates per two sidereal hours, Run IIb. . . 165

E.5 Number of $\ell+3$-jets $t \bar{t}$ candidates per two sidereal hours, Run IIb. . . 168

E.6 Number of $\ell+>3$-jets $t \bar{t}$ candidates per two sidereal hours, Run IIb. . 171

F.1 Fitted $f_{\mathrm{SME}}\left(\phi_{i}\right)$ (line) and observed $R_{i}$ (crosses), $e+>3$-jets $t \bar{t}$ candidates, for $\left(c_{R}\right)_{\mu \nu}=0$ : (a) $\left(c_{L}\right)_{X X}$, (b) $\left(c_{L}\right)_{X Y}$, (c) $\left(c_{L}\right)_{X Z},(\mathrm{~d})\left(c_{L}\right)_{Y Z} \cdot 176$

F.2 Fitted $f_{\mathrm{SME}}\left(\phi_{i}\right)$ (line) and observed $R_{i}$ (crosses), $e+>3$-jets $t \bar{t}$ candidates, for $\left(c_{L}\right)_{\mu \nu}=0$ : (a) $\left(c_{R}\right)_{X X}$, (b) $\left(c_{R}\right)_{X Y}$, (c) $\left(c_{R}\right)_{X Z}$, (d) $\left(c_{R}\right)_{Y Z}$.

F.3 Fitted $f_{\mathrm{SME}}\left(\phi_{i}\right)$ (line) and observed $R_{i}$ (crosses), $e+>3$-jets $t \bar{t}$ candidates, for $d_{\mu \nu}=0$ : (a) $c_{X X}$, (b) $c_{X Y}$, (c) $c_{X Z}$, (d) $c_{Y Z} \ldots \ldots 178$

F.4 Fitted $f_{\mathrm{SME}}\left(\phi_{i}\right)$ (line) and observed $R_{i}$ (crosses), $e+>3$-jets $t \bar{t}$ candidates, for $c_{\mu \nu}=0$ : (a) $d_{X X}$, (b) $d_{X Y}$, (c) $d_{X Z}$, (d) $d_{Y Z} \ldots \ldots . . . . \quad 179$

F.5 Fitted $f_{\mathrm{SME}}\left(\phi_{i}\right)$ (line) and observed $R_{i}$ (crosses), $\mu+>3$-jets $t \bar{t}$ candidates, for $\left(c_{R}\right)_{\mu \nu}=0$ : (a) $\left(c_{L}\right)_{X X}$, (b) $\left(c_{L}\right)_{X Y}$, (c) $\left(c_{L}\right)_{X Z}$, (d) $\left(c_{L}\right)_{Y Z}$.

F.6 Fitted $f_{\mathrm{SME}}\left(\phi_{i}\right)$ (line) and observed $R_{i}$ (crosses), $\mu+>3$-jets $t \bar{t}$ candidates, for $\left(c_{L}\right)_{\mu \nu}=0$ : (a) $\left(c_{R}\right)_{X X}$, (b) $\left(c_{R}\right)_{X Y}$, (c) $\left(c_{R}\right)_{X Z},(\mathrm{~d})\left(c_{R}\right)_{Y Z}$.

F.7 Fitted $f_{\mathrm{SME}}\left(\phi_{i}\right)$ (line) and observed $R_{i}$ (crosses), $\mu+>3$-jets $t \bar{t}$ candidates, for $d_{\mu \nu}=0$ : (a) $c_{X X}$, (b) $c_{X Y}$, (c) $c_{X Z}$, (d) $c_{Y Z} \ldots \ldots \ldots 2$

F.8 Fitted $f_{\mathrm{SME}}\left(\phi_{i}\right)$ (line) and observed $R_{i}$ (crosses), $\mu+>3$-jets $t \bar{t}$ candidates, for $c_{\mu \nu}=0$ : (a) $d_{X X}$, (b) $d_{X Y}$, (c) $d_{X Z}$, (d) $d_{Y Z} . \ldots 183$ 
F.9 Fitted $f_{\mathrm{SME}}\left(\phi_{i}\right)$ (line) and observed $R_{i}$ (crosses), $e+>3$-jets and $\mu+>3$ jets $t \bar{t}$ candidates, for $\left(c_{R}\right)_{\mu \nu}=0$ : (a) $\left(c_{L}\right)_{X X}$ (e+jets), (b) $\left(c_{L}\right)_{X X}$ $(\mu+$ jets $),(\mathrm{c})\left(c_{L}\right)_{X Y}(e+$ jets $),(\mathrm{d})\left(c_{L}\right)_{X Y}(\mu+$ jets $),(\mathrm{e})\left(c_{L}\right)_{X Z}(e+$ jets $)$, (f) $\left(c_{L}\right)_{X Z}\left(\mu+\right.$ jets), (g) $\left(c_{L}\right)_{Y Z}$ (e+jets), (g) $\left(c_{L}\right)_{Y Z}(\mu+$ jets $) \ldots \ldots 184$

F.10 Fitted $f_{\mathrm{SME}}\left(\phi_{i}\right)$ (line) and observed $R_{i}$ (crosses), $e+>3$-jets and $\mu+>3$ jets $t \bar{t}$ candidates, for $\left(c_{L}\right)_{\mu \nu}=0$ : (a) $\left(c_{R}\right)_{X X}$ (e+jets), (b) $\left(c_{R}\right)_{X X}$ $(\mu+$ jets $),(\mathrm{c})\left(c_{R}\right)_{X Y}(e+\mathrm{jets}),(\mathrm{d})\left(c_{R}\right)_{X Y}(\mu+\mathrm{jets}),(\mathrm{e})\left(c_{R}\right)_{X Z}(e+\mathrm{jets})$, (f) $\left(c_{R}\right)_{X Z}$ ( $\mu+$ jets $),(\mathrm{g})\left(c_{R}\right)_{Y Z}$ (e+jets), (g) $\left(c_{R}\right)_{Y Z}(\mu+$ jets $) . \ldots 185$

F.11 Fitted $f_{\mathrm{SME}}\left(\phi_{i}\right)$ (line) and observed $R_{i}$ (crosses), $e+>3$-jets and $\mu+>3$ jets $t \bar{t}$ candidates, for $d_{\mu \nu}=0$ : (a) $c_{X X}$ (e+jets), (b) $c_{X X}$ ( $\mu+$ jets), (c) $c_{X Y}$ (e+jets), (d) $c_{X Y}$ ( $\mu+$ jets), (e) $c_{X Z}$ (e+jets), (f) $c_{X Z}$ ( $\mu+$ jets $),(\mathrm{g})$ $c_{Y Z}(e+$ jets $),(\mathrm{g}) c_{Y Z}(\mu+$ jets $) . \ldots \ldots \ldots \ldots \ldots \ldots$

F.12 Fitted $f_{\mathrm{SME}}\left(\phi_{i}\right)$ (line) and observed $R_{i}$ (crosses), $e+>3$-jets and $\mu+>3$ jets $t \bar{t}$ candidates, for $c_{\mu \nu}=0$ : (a) $d_{X X}$ (e+jets), (b) $d_{X X}$ ( $\mu+$ jets), (c) $d_{X Y}$ (e+jets), (d) $d_{X Y}$ ( $\mu+$ jets), (e) $d_{X Z}$ (e+jets), (f) $d_{X Z}$ ( $\mu+$ jets $)$,

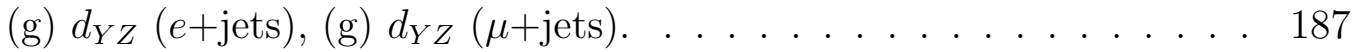




\section{Chapter 1}

\section{Introduction}

Physicists forge a deep connection with the world around us by seeking to understand the mechanisms by which it operates. Through centuries of observation and careful analysis, a broad-reaching and profoundly thorough quantitative description of these mechanisms has been developed. Disparate phenomena are connected by common underlying principles and symmetries. The analysis presented here seeks to test one of these fundamental tenets. Specifically, it is a search for a "preferred" direction in spacetime - something which would contradict the findings of physicists from Galileo to Einstein - but a direction which only applies to the top quark, the heaviest of the fundamental constituents of matter. The mathematical laws describing physical processes should be insensitive to the orientation and velocity of an experiment, and a violation of this principle is referred to as "Lorentz violation" (LV). Many tests of this principle have been carried out, but none so far have probed the regime of the top quark. We present a detailed discussion of the analysis published in Reference [1], the results of the first experimental investigation of Lorentz violation in the top 
quark sector.

This chapter will provide a general introduction to particle physics, to familiarize the reader with the concepts relevant to the analysis and the apparatuses used. The next section provides an overview of the Standard Model of Particle Physics, the description of the fundamental subatomic constituents of the known universe. Central to this analysis is the top quark, the heaviest of the constituents of matter, warranting a more in-depth discussion of its properties and the history of its discovery. Following this is a brief overview of modern experimental particle physics and the fantastic equipment needed for such research. The final section of the introduction discusses another fundamental concept of physics - the role of symmetry, particularly Lorentz symmetry.

The second chapter provides a more in-depth description the mathematics of the Standard Model and the mechanisms of Lorentz violation through the StandardModel Extension [2], the current limits and constraints on violation of this symmetry, and the facets accessible through a study of Lorentz violation in the top-quark sector. The third chapter discusses the unique signatures of Lorentz violation in the behavior of top quarks produced at particle colliders and details the theoretical derivation of our expectation for these signatures.

Chapters four through six describe the $\mathrm{D} \varnothing$ detector at Fermilab's Tevatron facility, along with the analysis and results of our search for Lorentz violation there. Chapter seven describes the expected sensitivity of this search at the ATLAS detector at CERN's Large Hadron Collider. Finally, chapter eight presents a discussion of the results and conclusions of this dissertation. 


\subsection{Overview of the Standard Model}

The mathematical framework which describes the fundamental constituents of nature and their interactions is called the Standard Model (SM). This theory does a remarkable job predicting the observed properties of the interacting matter and energy in the universe. To study and develop the Standard Model is to investigate the way the universe operates at the most fundamental level.

The top quark is the heaviest of the known fundamental particles. It is the most massive of the six quarks, the particles which bind together into the bulk of what we observe as matter. The top quark provides some unique opportunities to study aspects of the Standard Model and possible physics beyond it. Its large mass may mean that it plays an important role in the interactions between particles and the mechanism by which particles acquire mass.

\subsubsection{The Standard Model}

The quest for a deeper understanding of how the world around us is constructed has pushed the definition of what is truly fundamental to smaller and smaller scales. By the end of the 20th century results from investigations in a variety of fields of physics, both theoretical and experimental, had coalesced into the model of the fundamental constituents of matter and their interactions called the Standard Model. With this model we can peer deeper into the nature of the universe, beyond the levels of molecules, atoms, and atomic nuclei, into the interiors of protons and neutrons (Fig. 1.1).

In the regime of the subatomic, physical systems paradoxically exhibit properties 


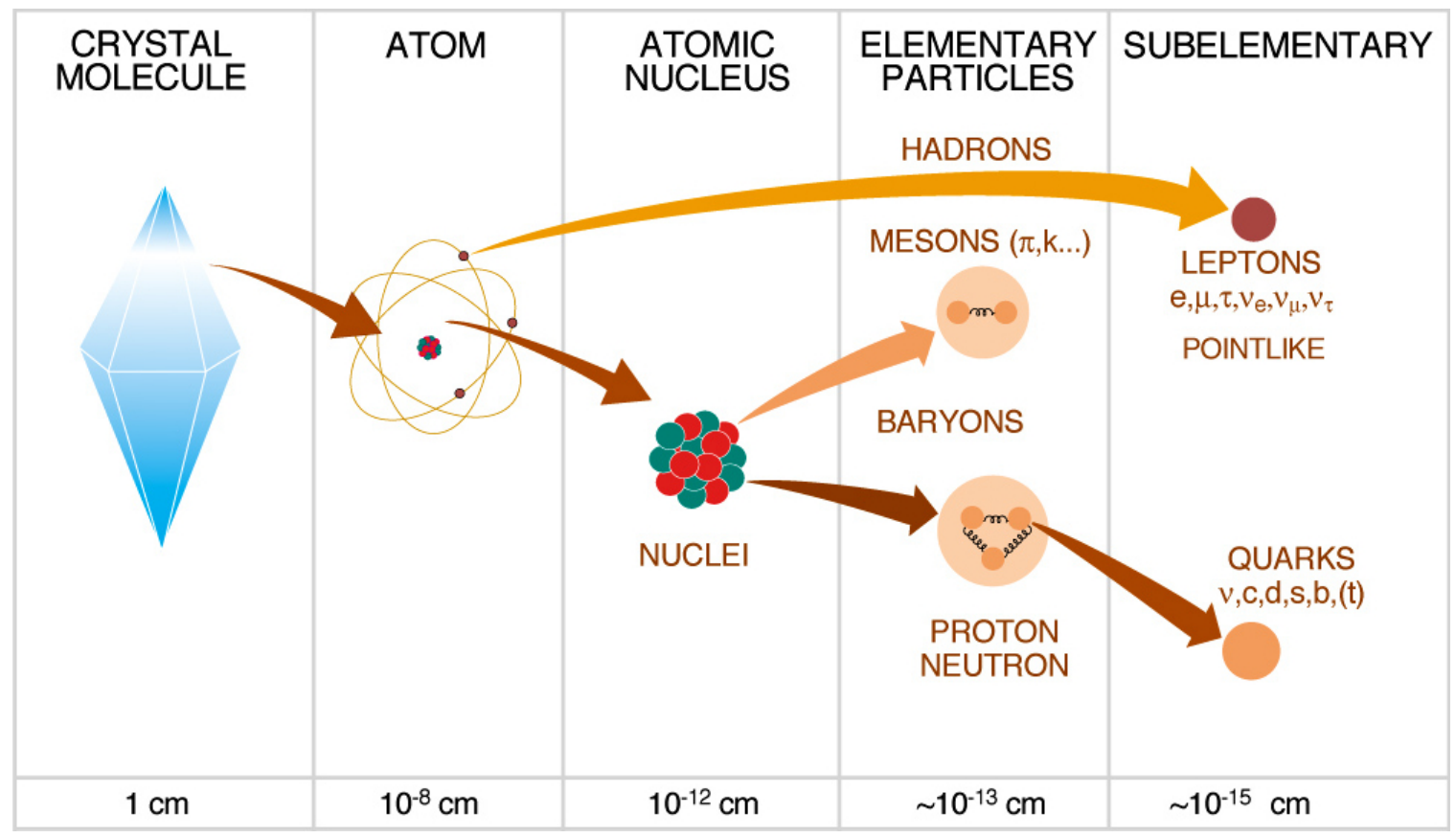

Figure 1.1: Zooming in on the structure of matter. (C) 1992 CERN)

of both particles (such as localization) and waves (including interference and diffraction). As a curious result, measurable quantities do not exist in a continuum but occur with discrete, "quantized" values. Thus, quantum mechanics plays a dominant role in describing the behavior of the fundamental building blocks of the universe. The Standard Model provides a rigorous mathematical framework within which these fundamental particles can be understood quantitatively. As we discuss below, all elements of the Standard Model - both the constituents which comprise matter and the forces mediating the interactions between them - are quantum objects, exhibiting this wave-particle duality. For simplicity, we refer to these quantum objects by the term "particle."

After decades of experimental discoveries and theoretical developments, what once 
appeared to be an entire "particle zoo" of dozens of particles has been distilled into a collection of a handful of truly fundamental particle species. These are divided into two major categories, based on an intrinsic quantum mechanical property called "spin." This is a property of the species of a particle, and is related not only to the classical concept of angular momentum but also to the statistical properties of each species. This quantum number divides the particles of the Standard Model into bosons (particles with integer spin - 0 or 1 units of the reduced Planck constant $\hbar)$ and fermions (particles with spin $\left.\frac{1}{2} \hbar\right)$. As a result of their collective statistical properties, the bosons act as mediators of the interactions between particles, and the fermions constitute matter.

The fermions of the Standard Model are further divided into two categories, dubbed "quarks" and "leptons." The familiar electron is an example of a lepton. This particle is found at everyday energies bound to atoms and molecules and is responsible for the chemical properties of substances. Within the Standard Model there are three charged flavors of lepton - the electron and its more massive cousins the muon and tau lepton. There also exist three related flavors of neutral leptons, the ghostly neutrinos.

The other category of fermions in the Standard Model are the quarks. These fundamental particles come in six flavors: down, up, strange, charm, bottom, and top. The first two, the down quark and the up quark, have the lowest mass and therefore are the most stable and common in the universe. The protons and neutrons at the nucleus of every atom are composed of a combination of three of these quarks ( $u+u+d$ for the proton and $u+d+d$ for the neutron). The more massive quarks play roles in more exotic particles and interactions which occur at higher energies. 
The fermions are grouped into three "generations." The lightest and most common quarks and leptons - the down and up quarks, the electron, and the electron neutrino - constitute the first generation. The strange quark, charm quark, muon, and muon neutrino share the same properties as their respective first-generation counterparts, but are more massive. They constitute the second generation. Similarly, the third generation fermions are the bottom and top quarks, the tau lepton, and the tau neutrino.

While the fermions constitute "matter," the bosons mediate the forces between them. By far the most familiar and intuitive of the constituents of the Standard Model, the photon transmits electromagnetic interactions between electrically charged particles. The electromagnetic spectrum, including radio and visible light, represents photons of varying energies. This interaction of electrically charged subatomic particles through the electromagnetic force is described through a combination of quantum mechanics and special relativity, a component of the Standard Model called quantum electrodynamics (QED).

Beyond electromagnetism, there are two other forces which play a role at the energy scales described by the Standard Model. The strong nuclear force binds quarks together and overcomes the electromagnetic repulsion of protons to hold atomic nuclei together. The quarks also carry an additional charge associated with this force that comes in one of three varieties. These three charges are dubbed "red," "green," and "blue," in analogy to colors and their mixing properties. Similar to the role that the photon plays in QED, a particle called the gluon is exchanged between particles carrying this color charge. Quantum chromodynamics (QCD) shares many properties with QED, but is complicated by the fact that the gluons themselves also carry color 
charge (while the photons do not carry electric charge). One consequence of this complication is that the force between quarks grows with the distance between them, permanently confining them into color-neutral groupings (e.g. red + blue + green). Composite particles made up of bound quarks, such as protons and neutrons, are called "hadrons." In this way, the internal structure of a hadron is akin to a soup of quarks and gluons all bound together. The quarks and gluons which make up hadrons are generally referred to as "partons."

Low-probability interactions, like the decays of relatively long-lived particles, are described by a third fundamental force, called the weak nuclear force. This force is actually mediated by three different bosons - the $W^{+}, W^{-}$, and $Z$. The $W$ bosons are themselves electrically charged, while the $Z$ boson is electrically neutral. Unlike the photon and gluon, the weak bosons are massive particles. As a result, the weak force tends to be short-ranged and feeble. Nonetheless, there are important similarities between the weak force and the electromagnetic force. So much so that in the late 1960s QED was extended into a unified theory of electromagnetism and the weak interaction. Electroweak theory describes the symmetry between the photon and the $W^{+}, W^{-}$, and $Z$ bosons. At very high energies, the interactions mediated by these four particles are indistinguishable. This symmetry is broken at low energies, attributing mass to the weak bosons while keeping the photon massless and thereby distinguishing the two forces.

The discussion so far has omitted the force of gravity. The hypothetical graviton, a boson with spin 2, is the mediator of gravitational force. However, while equally fundamental this interaction is certainly not equal in strength. Because of its prominent presence in everyday experience, one would expect that gravity should play an 
important role in the interaction of the particles of the Standard Model. However, the electromagnetic and strong forces are 36 to 38 orders of magnitude greater than gravity. Even the weak force is 25 orders of magnitude stronger. A simple observation demonstrates this disparity: the seemingly tiny strength of a common refrigerator magnet held in place by the force of electromagnetism is sufficient to overcome the gravitational force exerted on it by the entire mass of the Earth. Gravity is in fact so feeble relative to the other three forces that it is negligible in all calculations of processes involving the particles of the Standard Model.

Table 1.1: Particles of the Standard Model.

\begin{tabular}{|c|c|c|c|c|}
\hline \multirow{4}{*}{ Fermions } & \multicolumn{2}{|c|}{ quarks } & \multicolumn{2}{|r|}{ leptons } \\
\hline & down $(d)$ & up $(u)$ & electron $(e)$ & electron neutrino $\left(\nu_{e}\right)$ \\
\hline & strange $(s)$ & charm $(c)$ & muon $(\mu)$ & muon neutrino $\left(\nu_{\mu}\right)$ \\
\hline & bottom $(b)$ & top $(t)$ & $\operatorname{tau}(\tau)$ & tau neutrino $\left(\nu_{\tau}\right)$ \\
\hline \multirow{3}{*}{ Bosons } & \multicolumn{2}{|l|}{ gluon $(g)$} & & \\
\hline & \multicolumn{3}{|l|}{ photon $(\gamma)$} & \\
\hline & \multicolumn{4}{|c|}{ weak bosons $\left(W^{+}, W^{-}, Z\right)$} \\
\hline
\end{tabular}

Table 1.1 summarizes the constituents of the Standard Model. However, there is one additional component which is missing. In order for the mathematical theory to correctly describe their interactions, all particles must initially be massless. They then acquire the effective masses we observe through some interaction mechanism. The leading explanation, spearheaded by various theorists in the mid 1960s, generates the observed masses of the particles of the Standard Model through an interaction which breaks the symmetry between the electromagnetic and weak forces [3]. The particle associated with this mechanism, commonly called the "Higgs boson" after one of the leading investigators of its implications, remains the principal prediction of 
the Standard Model to be confirmed. In July of 2012, the ATLAS and CMS collaborations announced the discovery of a new boson with a mass of about $126 \mathrm{GeV} / \mathrm{c}^{2}$ that appears consistent with the expected signature of the Standard Model Higgs boson [4]. Continued analysis of future data will be necessary to measure its properties and conclusively determine whether this new particle is that predicted by the Higgs mechanism.

As the mathematical framework to describe the Standard Model was being developed, a curious feature emerged. In 1928 physicist Paul Dirac proposed a formulation to describe the interaction of fermions in a manner that is consistent with both quantum mechanics and special relativity [5]. This formulation, known as the Dirac equation, implies the existence of a new form of matter - fermions identical to the familiar ones described above, but with opposite charge. When a particle of normal matter and its "antimatter" counterpart interact they annihilate, converting their energy into photons.

In 1932, just a few years after this revolutionary theoretical framework was proposed, the first experimental discovery of an antiparticle was made [6]. Antielectrons (dubbed "positrons") were first observed in the products of cosmic ray interactions, and later found to be an integral part of certain radioactive decays. Many other antiparticles have since been discovered, built from the antiparticle partners of the fermions of the Standard Model. Recently, whole atoms of antihydrogen built from antiprotons and positrons have been fleetingly created and observed in the laboratory [7]. One of the great mysteries of modern physics is to explain why the universe is filled with matter while there is so little naturally-occurring antimatter.

Finally, we have described the elementary constituents of the Standard Model of 
Particle Physics. Fermions (and their antiparticle partners) comprise the matter the "stuff" - of the universe, while bosons mediate the fundamental forces between them - electromagnetism, the strong force, and the weak force. Three quarks (or three antiquarks) bind together to form a class of matter known as baryons. One quark can pair up with one antiquark to form another class called mesons. The up and down quarks bind together in groups of three to form the common and abundant protons and neutrons, which comprise the nuclei of atoms. Electrons join these nuclei to give rise to the chemical properties of the elements. The ghostly neutrinos only interact via the weak interaction, but play roles in nuclear interactions and perhaps even in the evolution of the universe. The quarks and antiquarks of the second and third generations play important roles in more exotic and higher-energy interactions.

Still, the Standard Model is not yet complete. While it has proven wildly successful in describing the fundamental constituents of the universe and their interactions, there remain discrepancies between prediction and observation. The fact that neutrinos oscillate between different flavors (generations) is nowhere predicted by the Standard Model. Although the Standard Model predicts only a tiny asymmetry between matter and antimatter, there must be a strong fundamental difference between the two. Equal amounts should have existed at the beginning of the universe and would have annihilated completely to photons, yet our universe appears to be dominated by matter. What's more, increasingly compelling astrophysical observations indicate that the particles of the Standard Model can only account for $4 \%$ of the contents of the universe, with the rest some new form of "dark matter" and "dark energy" about which we know little and the Standard Model says nothing. There is much room for physics beyond the Standard Model. Nature continues to tantalize with many 
mysteries to solve and the promise of many surprises to come.

\subsubsection{The top quark}

With the Standard Model neatly arranged in tabular form (Table 1.1), we now have a nice and succinct pool from which to build much of the universe. Familiar matter is built from the first generation particles; the proton consists of two up quarks and one down quark; the neutron is built from two down quarks and one up quark; and electrons orbit nuclei made up of protons and neutrons.

The particles of the second generation are "heavy cousins" of the first generation, and the third generation particles are more massive still. Because of this, they do not play a significant role in the behavior of matter at ordinary energies. They do participate in important ways in high energy subatomic phenomena, though. Of particular interest to the analysis presented in this dissertation is the top quark.

Last of the quarks to be discovered, the top quark proved elusive for years. After the discovery of the bottom quark in 1977 by the E288 experiment at Fermilab [8], it became clear that there was a third generation of quarks. (There is good reason to believe there are only three generations of subatomic particles, but theorists remain open to the possibility of more.) While the top quark was expected to be more massive than the bottom quark, physicists were surprised by repeated failures to create it in the laboratory with ever more energetic experiments.

Accelerators at Fermilab and CERN raced through the 1980s to make the discovery. Although the UA1 and UA2 experiments at CERN's Super Proton Synchrotron discovered the massive $W$ and $Z$ bosons in 1983 [9][10], the top quark remained undiscovered. Armed with a lower limit on its mass from CERN, physicists at Fer- 
milab were able to use the higher energy reach of the Tevatron accelerator to probe larger masses. Finally, in 1995 physicists at the CDF and DØ experiments jointly announced the discovery of the top quark [11]. Its mass was measured at approximately $175 \mathrm{GeV} / c^{2}$, making it a subatomic particle as massive as an entire atom of tungsten.

The large mass of the top quark makes it fertile ground for investigations into a number of phenomena. The top quark decays almost exclusively to a $\mathrm{W}$ boson and a bottom quark, in a process which happens much faster than the characteristic timescale for strong interactions. Unlike the other quarks, which bind together into hadrons well before they can decay, the top quark decays essentially as a free quark, providing insights into the behavior of unconfined quarks. Furthermore, because it is so massive (almost twice that of the $W$ and $Z$ bosons), the top quark also may play an important role in the behavior of the weak force carriers and the breaking of electroweak symmetry. In fact, important properties of the Higgs boson can be extrapolated from precise measurements of the top quark mass.

\subsection{Modern experimental collider physics}

Probing the fundamental constituents of the universe is not straightforward. The Standard Model neatly describes the interactions between fermions and bosons, but the unimaginably tiny length scales and high energies at which the most interesting of these interactions occur make these subjects difficult to observe directly.

In order to create the conditions in the laboratory in which Standard Model processes can be practically studied, subatomic particles are accelerated to nearly the 
speed of light and collided head-on. Through mass-energy equivalence $\left(E=m c^{2}\right)$ the large kinetic energy of these particles can be transformed into different, massive particles like the weak bosons and the fermions of the second and third generations. The various possible transformations all occur with differing probability, dependent on the masses of the participating particles and the strength of their interactions. Thus, an extremely large number of collision events must be analyzed in order to make any statistically-significant measurement of particle properties.

The initial-state particles interact, perhaps scattering or perhaps annihilating, and final-state particles exit the interaction. This process is called the "hard scatter." Many collider experiments have employed electrons and positrons as the initial particles driven together toward the hard scatter. Accelerating such low-mass particles to higher energies becomes progressively more difficult as the added kinetic energy is quickly radiated away. The largest modern collider experiments instead accelerate protons, which suffer less from this effect at comparable energies. Of course, the resulting collisions are somewhat more complicated. Rather than the entire proton interacting with its collision partner, a quark or a gluon forming the internal structure of the proton interacts with a quark or gluon from the other incident proton.

The initial-state particles interact probabilistically to produce an intermediate state, which then produces two or more final-state particles. The properties of the final-state particles - e.g. their flavors, masses, spins, energies, and directions - give information about the intermediate state and the details of the interactions involved.

Often final-state particles from the hard scatter are not physically observable. Quarks only manifest themselves as hadrons through a process called "hadronization." These and other particles may also be unstable, decaying into several less massive 
particles before traveling far from the point where they were produced. This collection of associated final state particles is referred to as a "jet," and is distributed over an extended region of the detector. Measurement of the final state particles from the hard scatter is therefore a complicated task involving the detection of a large number of different particle species and careful extrapolation to reconstruct the particles from which they all originated.

Measurement of collision events is facilitated by sophisticated particle physics detectors consisting of many layers of arrays of various sensors designed to detect the passage of a variety of particle species. One such detector experiment is called ATLAS, consisting of a number of typical sensor types. Fig. 1.2 illustrates a crosssectional slice of the ATLAS detector, showing common particles produced in collision events and the subdetectors designed to detect each type. There are two major classes of subdetectors - tracking systems and calorimeters.

Trackers consist of a large number of small sensors designed to detect the passage of electrically charged particles with a fine position resolution. Collections of "hits" within the tracking volume are combined to reconstruct the path of individual charged particles. Tracking detectors are usually situated inside a solenoidal magnetic field oriented parallel to the collision axis. Because the path of a moving charged particle is deflected perpendicular to the direction of the magnetic field in a way that depends on its charge and momentum, the curvature of the resulting helical paths can be measured to calculate each particle's momentum.

Calorimeters are designed such that particles deposit all of their kinetic energy within the detector volume, thereby providing a measurement of this energy. Particles such as electrons and photons interact with the electrons in the calorimeter material, 


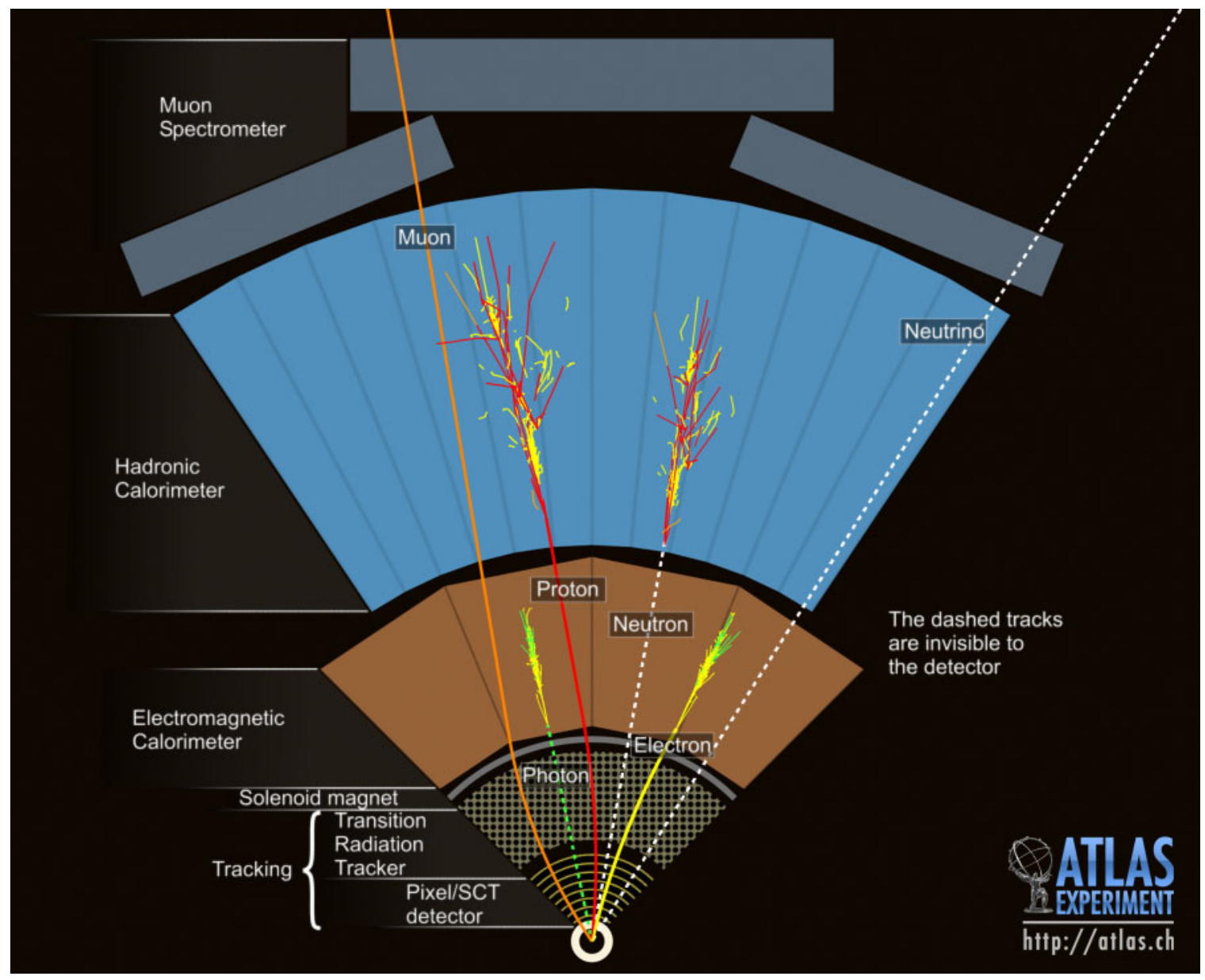

Figure 1.2: Various subdetectors of the ATLAS detector and the particles they specialize in measuring. (ATLAS Experiment (C) 2012 CERN)

and electromagnetic calorimeters are optimized to capture them. Hadrons such as protons and neutrons interact principally through elastic collisions with the nuclei of the calorimeter material, so a different design is used for hadronic calorimeters. Quarks and gluons from the underlying event evolve into jets of final-state particles which deposit their energy over a extended regions of the calorimeters and are reconstructed by associating individual energy depositions into clusters. 
Muons produced in typical collision events occur with energies at which they are so-called "minimally-ionizing particles" (MIP), meaning they lose very little energy as they traverse the various materials making up the detector. They pass through the electromagnetic and hadronic calorimeter systems almost unaffected. Outer layers of additional tracking detectors extend the measured trajectories of muons, both identifying them uniquely as muons and providing extra accuracy in the measurement of their momenta.

\section{$1.3 \quad$ Symmetry}

We have already hinted at the role that symmetry plays in particle physics. The organization of the particle zoo into groups of related particles pointed toward some underlying symmetry between members of these groups. This symmetry led to the development of the quark model, later experimentally verified. The behavior of the weak and electromagnetic forces would be radically different from what we observe were it not for electroweak symmetry breaking. Through the Higgs mechanism, the symmetry between the photon and the $W^{+}, W^{-}$, and $Z$ bosons is broken. Charge reversal (flipping the electric charge of the electron and positron, for example), parity (doing physics in a mirror), and time reversal are three other important symmetries which play crucial roles in a variety of subatomic processes.

What is meant by symmetry, in the context of physics? A property of a system is symmetric under some transformation if that transformation leaves that property unchanged. The shape of a baseball is rotationally symmetric, because rotations of the baseball leave its shape unchanged. However, the rotational symmetry of an 
American football is broken because one axis is elongated relative to the other two. As an example more specific to particle physics, electromagnetism is symmetric under reversal of charges. Opposite electric charges always attract and like-signed electric charges always repel.

The deep connection between physics and symmetry was elucidated by the early 20th century mathematician Emmy Noether. Simply put, Noether's theorem states that any continuous symmetry of a physical system has an associated conservation law. This simple statement has profound implications. The translational symmetry of physics - the fact that the laws of physics are the same independent of the location where an experiment is performed - leads directly to the conservation of momentum. The unchanging nature of these laws over time leads directly to the conservation of energy. The independence of the laws of nature on the orientation of an experiment (in the absence of a symmetry-breaking effect such as the Earth's gravity) leads to the conservation of angular momentum.

Symmetries have direct consequences on the laws of nature, and broken symmetries can lead to profound effects. The laws of physics should be immutable under so-called "CP-symmetry," the product of charge reversal symmetry (swapping particles for their antiparticles) and parity symmetry (flipping all directions as through a mirror). If this symmetry were broken strongly enough, it could provide a sufficient mechanism to explain the aforementioned excess of matter over antimatter in the universe. The Standard Model exhibits some CP violation, but far too weakly to provide the observed excess. The search for additional mechanisms for strong CP violation is one of the major challenges being addressed by modern particle physics. 


\subsubsection{Lorentz symmetry}

One particular symmetry is of interest in this analysis. Lorentz symmetry arises from the laws of special relativity and posits that the laws of physics are invariant regardless of the inertial (non-accelerating) reference frame in which they are observed. The Lorentz transformation mixes rotations between reference frames with boosts between reference frames moving with constant relative velocity. The constancy of the laws of physics under Lorentz transformations is fundamental to our understanding of the universe.

The Lorentz transformation was originally derived by physicist Hendrick Lorentz, who sought to explain how the speed of light was found to be the same for all observers, independent of their reference frame. Einstein later re-derived this transformation as a natural mathematical statement stemming from the consequences of the revolutionary postulates in his special theory of relativity [12]:

1. The laws by which the states of physical systems undergo change are not affected, whether these changes of state be referred to the one or the other of two systems of co-ordinates in uniform translatory motion.

2. Any ray of light moves in the "stationary" system of co-ordinates with the determined velocity $c$, whether the ray be emitted by a stationary or by a moving body.

At the time of this development, the fact that the laws of physics should be the same as measured by observers in any inertial reference frame was not a new development. That all such observers measure the same speed for light was. Einstein accepted these postulates and reconciled them into the special theory of relativity. 
The resulting Lorentz transformation describes how the coordinates in one inertial reference frame correspond to the coordinates in another inertial reference frame.

Perhaps the most profound implication of this transformation is the fact that it mixes the space and time coordinates of events in different reference frames. In contrast to the long held beliefs of classical physics time can no longer be considered a constant, and intervals between events (say, the ticks of a clock) are measured differently by different observers in relative uniform motion. Some of the resulting phenomena, including length contraction and time dilation, while fascinating and deserving of a thorough discussion, are beyond the scope of this paper.

In order to take into account the fact that time and spatial coordinates transform together under special relativity, the conventional three-element vector denoting position is extended to include the time coordinate. One common notation convention for a "four vector" denoting position is $(t, x, y, z)$. Similarly, the four vector denoting momentum is $\left(E, p_{x}, p_{y}, p_{z}\right)$. Eq. (1.1) is the matrix parametrizing the rotation-free Lorentz transformation of these four vectors, called a "boost."

$$
L_{\beta}=\left(\begin{array}{cccc}
\gamma & -\beta_{x} \gamma & -\beta_{y} \gamma & -\beta_{z} \gamma \\
-\beta_{x} \gamma & 1+(\gamma-1) \frac{\beta_{x}^{2}}{\beta^{2}} & (\gamma-1) \frac{\beta_{x} \beta_{y}}{\beta^{2}} & (\gamma-1) \frac{\beta_{x} \beta_{z}}{\beta^{2}} \\
-\beta_{y} \gamma & (\gamma-1) \frac{\beta_{x}}{\beta^{2}} & 1+(\gamma-1) \frac{\beta_{y}^{2}}{\beta^{2}} & (\gamma-1) \frac{\beta_{y} \beta_{z}}{\beta^{2}} \\
-\beta_{z} \gamma & (\gamma-1) \frac{\beta_{z} \beta_{x}}{\beta^{2}} & (\gamma-1) \frac{\beta_{z} \beta_{y}}{\beta^{2}} & 1+(\gamma-1) \frac{\beta_{z}^{2}}{\beta^{2}}
\end{array}\right) .
$$

The factor $\vec{\beta}=\vec{v} / c$ is the relative velocity of the new reference frame, expressed as a fraction of the speed of light $c$. The Lorentz factor $\gamma$ is defined as

$$
\gamma=\frac{1}{\sqrt{1-\beta^{2}}} .
$$


To complete our mathematical description of the transformation between reference frames, we consider the contribution from rotations. A general rotation in three dimensions can be constructed by successive rotations about one Cartesian axis at a time. The resulting matrix is a product of some combination of three rotation matrices, $R_{x}(\phi), R_{y}(\theta)$, and $R_{z}(\psi)$. Each acts on the spatial components of a four vector (since time is only affected by boosts and not spatial rotations).

$$
\begin{aligned}
& R_{x}(\phi)=\left(\begin{array}{ccc}
1 & 0 & 0 \\
0 & \cos (\phi) & -\sin (\phi) \\
0 & \sin (\phi) & \cos (\phi)
\end{array}\right), \\
& R_{y}(\theta)=\left(\begin{array}{ccc}
\cos (\theta) & 0 & -\sin (\theta) \\
0 & 1 & 0 \\
\sin (\theta) & 0 & \cos (\theta)
\end{array}\right), \\
& R_{z}(\psi)=\left(\begin{array}{ccc}
\cos (\psi) & \sin (\psi) & 0 \\
-\sin (\psi) & \cos (\psi) & 0 \\
0 & 0 & 1
\end{array}\right) .
\end{aligned}
$$

A Lorentz transformation between different inertial reference frames can therefore be constructed as an appropriate product of Eqs. (1.1), (1.3)-(1.5).

Violation of Lorentz symmetry would profoundly alter our understanding of the nature of space and time. It would imply that there is a special reference frame or "direction" in spacetime. As an example from particle physics, the probability of a subatomic process (like $q \bar{q} \rightarrow t \bar{t}$ ) to occur could depend on the orientation of that process with respect to this special direction. Such an observation would contradict 
the theory of special relativity and overthrow one of the most fundamental tenets of physics.

\subsubsection{CPT symmetry}

A second symmetry of nature relevant to this analysis is called "CPT symmetry." The CPT theorem states that the physical laws governing the dynamics of particles are invariant under the simultaneous inversion of three different symmetries. Charge conjugation, C, reverses the charges of a particle, effectively transforming it into its antiparticle. The parity transformation, $\mathrm{P}$, reverses the sign of the three spatial coordinates, analogous to considering the mirror image of a system and effectively reversing the direction of vectors like position and linear momentum. While the universe at macroscopic scales does not exhibit T symmetry (the reversal of the flow of time), a fact demanded by the second law of thermodynamics, the dynamic laws of physics are generally symmetric under reversal of the sign of the time coordinate.

While each of the $\mathrm{C}, \mathrm{P}$, and $\mathrm{T}$ symmetries is independently conserved by the electromagnetic, gravitational, and strong forces, processes involving the weak force are known to violate all three of these symmetries separately. The unique feature of the weak force is that weak interactions occur with different strength depending on a particle's "chirality," the relative alignment of its spin state to the direction of its momentum. This force then violates, for example, $\mathrm{P}$ symmetry. Under a $\mathrm{P}$ transformation a right-handed electron (one whose spin is aligned with its momentum) would become a left-handed positron, which interacts weakly with a different strength than a right-handed electron.

Although all three of these symmetries are known to be violated separately by 
the weak interaction, the laws of physics appear to remain invariant under their simultaneous application. This equates to a universe in which all particles were swapped for their antiparticles, all positions and momenta were mirror reversed, and the flow of time was flipped. Violation of CPT symmetry could be observable in a particle physics experiment through differences between the decay rate of a process and its antiparticle analog (e.g. $q b \rightarrow q^{\prime} t$ and $\bar{q} \bar{b} \rightarrow \bar{q}^{\prime} \bar{t}$. This would have deep implications for the compatibility of quantum mechanics with special relativity, and would in fact imply violation of Lorentz symmetry as well [13]. 


\section{Chapter 2}

\section{The Standard-Model Extension}

Among the foundational principals of modern physics are the Lorentz and CPT symmetries. Physical processes are invariant under transformations between inertial reference frames and under the simultaneous reversal of charge, parity, and the direction of time. Because of their importance and fundamental nature in the description of the universe, scientific prudence demands these tenets be rigorously tested. In order to do so, we require a quantitative means of predicting the results of experiments as governed by the expected laws of physics, as well as under the assumption of their violation.

At its core, physics is built using the language of mathematics. The Standard Model of Particle Physics can be described qualitatively at length (as in the previous chapter), or in a rigorous quantitative manner using advanced mathematics. The Standard Model is relatively succinctly expressed through the formalism of Lagrangian mechanics, whereby all possible physical processes are described by various terms in a Lagrange density. The resulting dynamics of interacting particles can be 
derived from this Lagrange density through various analytical and numerical techniques.

The Standard Model is constructed under the explicit assumption of both Lorentz and CPT invariance, excluding any process which violates either symmetry. However, such processes can be reincorporated into the SM Lagrangian using a phenomenological framework known as the Standard-Model Extension (SME) [2]. This framework adds all possible Lorentz- and CPT-violating terms to the SM Lagrange density. By scaling each of these terms with an appropriate coefficient, the SME can describe these contributions in a model-independent way. Expectations for particle interactions can then be calculated under the assumption of Lorentz or CPT violation in various forms, independent of the specific mechanisms that may give rise to those violations.

In this chapter we outline the mathematical form of the Standard Model and describe the contributions from the SME which directly effect the top quark. We also provide a brief discussion of some of the searches for Lorentz and CPT violation in other particle sectors conducted utilizing the SME framework.

\subsection{The Standard Model Lagrangian}

The Standard Model is a quantum field theory, with elements represented by fields and a Lagrange density describing the interactions between these fields. From the Lagrange density, the dynamics of particles can be calculated. A detailed mathematical development of the SM can be found at Reference [14]; we seek only to present a brief description of its mathematical form here. 
Each fermion is represented by a Dirac spinor, a mathematical object whose four components are fields corresponding to the two spin states of both the fermion and its antiparticle. Bosons are represented by vector fields which couple to the various charges of the fermions. Gauge invariance, the freedom to multiply these boson fields by an arbitrary phase without effecting the dynamics, restricts the forms that the boson fields can take.

The symmetries of each interaction further specify the forms of the gauge boson fields, and group theory is employed in the construction of the SM Lagrangian. The $U(1)$ symmetry group describes the forms that the photon field can take, with its symmetry under electric charge reversal. The freedom to transform among the three color charges means the gluon field is best represented by the $S U(3)$ symmetry group. A peculiar feature of the weak interaction is that it acts differently on left- and righthanded fermions. A particle's handedness, or "chirality," is the combination of its spin state with the direction of its momentum. The two chiralities effectively have different weak charges, and this feature is represented by the $S U(2)$ symmetry group.

The electromagnetic and weak forces are unified into four electroweak gauge boson fields, and the addition of a scalar field is the simplest method of inducing electroweak symmetry breaking (e.g. the Higgs mechanism). The relevant symmetry groups are $S U(2)_{L}$, acting on the left-handed chirality, and $U(1)_{Y}$, coupling to a combination of electric charge and chirality called "hypercharge" $(Y)$. Together with the strong interaction, the combined gauge symmetry of the Standard Model is then

$$
S U(3)_{c o l o r} \otimes S U(2)_{L} \otimes U(1)_{Y} .
$$

The observed left-right asymmetry of the electroweak interaction makes organiza- 
tion of the fermion fields into left-handed doublets and right-handed singlets a natural formulation. We have

$$
\begin{aligned}
& L_{A}=\left(\begin{array}{c}
\left(\nu_{e}\right)_{L} \\
e_{L}
\end{array}\right),\left(\begin{array}{c}
\left(\nu_{\mu}\right)_{L} \\
\mu_{L}
\end{array}\right),\left(\begin{array}{c}
\left(\nu_{\tau}\right)_{L} \\
\tau_{L}
\end{array}\right) \\
& R_{A}=e_{R}, \mu_{R}, \tau_{R}
\end{aligned}
$$

for the lepton fields (where $A$ is the generation index), and

$$
\begin{aligned}
Q_{A} & =\left(\begin{array}{l}
u_{L} \\
d_{L}
\end{array}\right),\left(\begin{array}{l}
c_{L} \\
s_{L}
\end{array}\right),\left(\begin{array}{l}
t_{L} \\
b_{L}
\end{array}\right) \\
U_{A} & =u_{R}, c_{R}, t_{R} \\
D_{A} & =d_{R}, s_{R}, b_{R}
\end{aligned}
$$

for the quark fields. Because the neutrinos only interact via the $S U(2)_{L}$ interaction, their right-handed chirality states are absent from the Standard Model. Although experimental evidence, including the observation of flavor oscillations, has indicated that neutrinos have a small mass (and therefore exhibit right-handed chirality in appropriately boosted reference frames), within the Standard Model they are treated as massless and only the left-handed fields are included.

The gauge boson fields are $G_{\mu}^{a}(a=1, \ldots, 8), W_{\mu}^{i}(i=1,2,3)$, and $B_{\mu}$, corresponding to the $S U(3)_{c o l o r}, S U(2)_{L}$, and $U(1)_{Y}$ symmetries, respectively. The associated gauge invariant field strength tensors are 


$$
\begin{aligned}
G_{\mu \nu}^{a} & =\partial_{\mu} G_{\nu}^{a}-\partial_{\nu} G_{\mu}^{a}+g_{s} f^{a b c} G_{\mu}^{b} G_{\nu}^{c}, \\
W_{\mu \nu}^{i} & =\partial_{\mu} W_{\nu}^{i}-\partial_{\nu} W_{\mu}^{i}+g \epsilon^{i j k} W_{\mu}^{j} W_{\nu}^{k}, \\
B_{\mu \nu} & =\partial_{\mu} B_{\nu}-\partial_{\nu} B_{\mu} .
\end{aligned}
$$

The $W$ and gluon tensors include self-interaction terms, controlled by the appropriate coupling strengths $g$ and $g_{s}$ and mixed by the respective structure constants $\epsilon^{i j k}$ and $f^{a b c}$ of the corresponding symmetry groups. The Lorentz indices $\mu$ and $\nu$ label components of the relevant four vector $(t, x, y$, or $z)$. For notational brevity, we employ the Einstein summation convention, where multiplication of two terms with the same index implies a summation of the product over all values of the index.

The introduction of a doublet of scalar fields of the form

$$
\phi=\left(\begin{array}{l}
\phi^{+} \\
\phi^{0}
\end{array}\right)
$$

provides a mechanism for spontaneous electroweak symmetry breaking. The interaction of the $W$ and $B$ fields with this doublet mixes them, giving rise to the familiar $W^{+}, W^{-}, Z$, and photon $(A)$ fields.

$$
\begin{aligned}
A_{\mu} & =\cos \theta_{W} B_{\mu}+\sin \theta_{W} W_{\mu}^{3} \\
Z_{\mu} & =\sin \theta_{W} B_{\mu}-\cos \theta_{W} W_{\mu}^{3} \\
W_{\mu}^{ \pm} & =\frac{1}{\sqrt{2}}\left(W_{\mu}^{1} \mp i W_{\mu}^{2}\right),
\end{aligned}
$$

with weak mixing angle 


$$
\cos \theta_{W}=\frac{m_{W}}{m_{Z}} .
$$

The full Standard Model Lagrange density (before explicit electroweak symmetry breaking) thus has the form

$$
\begin{aligned}
\mathcal{L}= & -\frac{1}{4} W_{\mu \nu}^{i} W_{i}^{\mu \nu}-\frac{1}{4} B_{\mu \nu} B^{\mu \nu}-\frac{1}{4} G_{\mu \nu}^{a} G_{a}^{\mu \nu} \\
& +\bar{L}_{A} \gamma^{\mu}\left(i \partial_{\mu}+\frac{g}{2} \tau_{i} W_{\mu}^{i}-\frac{g^{\prime}}{2} B_{\mu}\right) L_{A} \\
& +\bar{R}_{A} \gamma^{\mu}\left(i \partial_{\mu}-g^{\prime} B_{\mu}\right) R_{A} \\
& +\bar{Q}_{A} \gamma^{\mu}\left(i \partial_{\mu}-\frac{g}{2} \tau_{i} W_{\mu}^{i}+\frac{g^{\prime}}{6} B_{\mu}+\frac{g_{s}}{2} \lambda_{a} G_{\mu}^{a}\right) Q_{A} \\
& +\bar{U}_{A} \gamma^{\mu}\left(i \partial_{\mu}+\frac{2}{3} g^{\prime} B_{\mu}+\frac{g_{s}}{2} \lambda_{a} G_{\mu}^{a}\right) U_{A} \\
& +\bar{D}_{A} \gamma^{\mu}\left(i \partial_{\mu}-\frac{1}{3} g^{\prime} B_{\mu}+\frac{g_{s}}{2} \lambda_{a} G_{\mu}^{a}\right) D_{A} \\
& +\left|\left(i \partial_{\mu}-\frac{g}{2} \tau_{i} W_{\mu}^{i}-\frac{g^{\prime}}{2} B_{\mu}\right) \phi\right|^{2}+\mu^{2} \phi^{\dagger} \phi-\lambda\left(\phi^{\dagger} \phi\right)^{2} \\
& -\left(y_{A B}^{L} \bar{L}_{A} \phi R_{B}+y_{A B}^{D} \bar{Q}_{A} \phi D_{B}-y_{A B}^{U} \bar{Q}_{A} i \tau_{2} \phi^{*} U_{B}+\text { h.c. }\right) .
\end{aligned}
$$

The constants $g, g^{\prime}$, and $g_{s}$ parametrize the coupling strength between fermions and the bosons mediating the weak isospin, hypercharge, and strong interactions, respectively. The four Dirac matrices $\gamma^{\mu}$ mix components of the fermion spinors, the $\tau_{i}$ are the three Pauli matrices (generators of the $S U(2)$ symmetry group), and $\lambda_{a}$ are the eight Gell-Mann matrices (generators of $S U(3)$ ). The $y$ matrices combine the Yukawa couplings of the various fermion fields to the scalar doublet $\phi$ with additional coupling terms that mix generations. The scalar potential $\mu^{2} \phi^{\dagger} \phi-\lambda\left(\phi^{\dagger} \phi\right)^{2}$ contains two free parameters $\mu$ and $\lambda$ which describe its shape and are intimately connected 
to the phenomena which emerge from the breaking of electroweak symmetry (for example, the masses of the weak and Higgs bosons). There are of course many more subtleties in this Lagrange density and deep implications of its terms that are beyond the scope of this discussion.

\subsection{SME addition to the top quark sector}

The Standard-Model Extension adds a variety of terms to Eq. (2.8) in order to introduce violation of Lorentz and CPT symmetry. Each of these terms is controlled by a coefficient describing its relative contribution to SM processes. Because the SME is a purely phenomenological framework, incorporating these violations in a modelindependent manner, these coefficients are not constrained to be the same for different particle species.

A full list of terms within the minimal SME can be found in tables XXIX and XXX of reference [15]. The SM Lagrangian can be extended by the addition of an

SME contribution $\left(\mathcal{L}_{\mathrm{SME}}\right)$. The terms in this contribution relevant to the quark sector are 


$$
\begin{aligned}
\mathcal{L}_{\mathrm{SME}}= & \left(a_{Q}\right)_{\mu A B} \bar{Q}_{A} \gamma^{\mu} Q_{B} \\
& +\left(a_{U}\right)_{\mu A B} \bar{U}_{A} \gamma^{\mu} U_{B} \\
& +\left(a_{D}\right)_{\mu A B} \bar{D}_{A} \gamma^{\mu} D_{B} \\
& +\left(c_{Q}\right)_{\mu \nu A B} \bar{Q}_{A} \gamma^{\mu}\left(i \partial^{\nu}-\frac{g}{2} \tau^{i} W_{i}^{\nu}+\frac{g^{\prime}}{6} B^{\nu}+\frac{g_{s}}{2} \lambda^{a} G_{a}^{\nu}\right) Q_{B} \\
& +\left(c_{U}\right)_{\mu \nu A B} \bar{U}_{A} \gamma^{\mu}\left(i \partial^{\nu}+\frac{2}{3} g^{\prime} B^{\nu}+\frac{g_{s}}{2} \lambda^{a} G_{a}^{\nu}\right) U_{B} \\
& +\left(c_{D}\right)_{\mu \nu A B} \bar{D}_{A} \gamma^{\mu}\left(i \partial^{\nu}-\frac{1}{3} g^{\prime} B^{\nu}+\frac{g_{s}}{2} \lambda^{a} G_{a}^{\nu}\right) D_{B} .
\end{aligned}
$$

The $a_{\mu}$-type coefficients have mass dimension 1 and control terms which are odd under CPT reversal, while the unitless $c_{\mu \nu}$-type coefficients control CPT-even terms. In order to perform a model-independent investigation of the contribution from these terms to processes involving top quarks, we consider the SME coefficients to be nonzero only when $A, B=3,3$.

\subsection{Reference frames}

One of the implications of Lorentz violation is a dependence of physical processes on the choice of inertial reference frame. In order to compare results from various experiments, we must choose a standard reference frame in which we express our observations. The coefficients of the Lorentz- and CPT-violating terms in the SME are defined in the canonical Sun-centered reference frame (Fig. 2.1) [15]. This convention provides a suitably constant reference frame over the lifetime of Earth and near-Earth based experiments, varying only on the timescale of a century. We employ the convention of using upper-case Lorentz indices $\mu, \nu \in\{T, X, Y, Z\}$ to denote co- 
ordinates in the Sun-centered reference frame and lower-case indices $\alpha, \beta \in\{t, x, y, z\}$ for coordinates in the reference frame of an experiment.

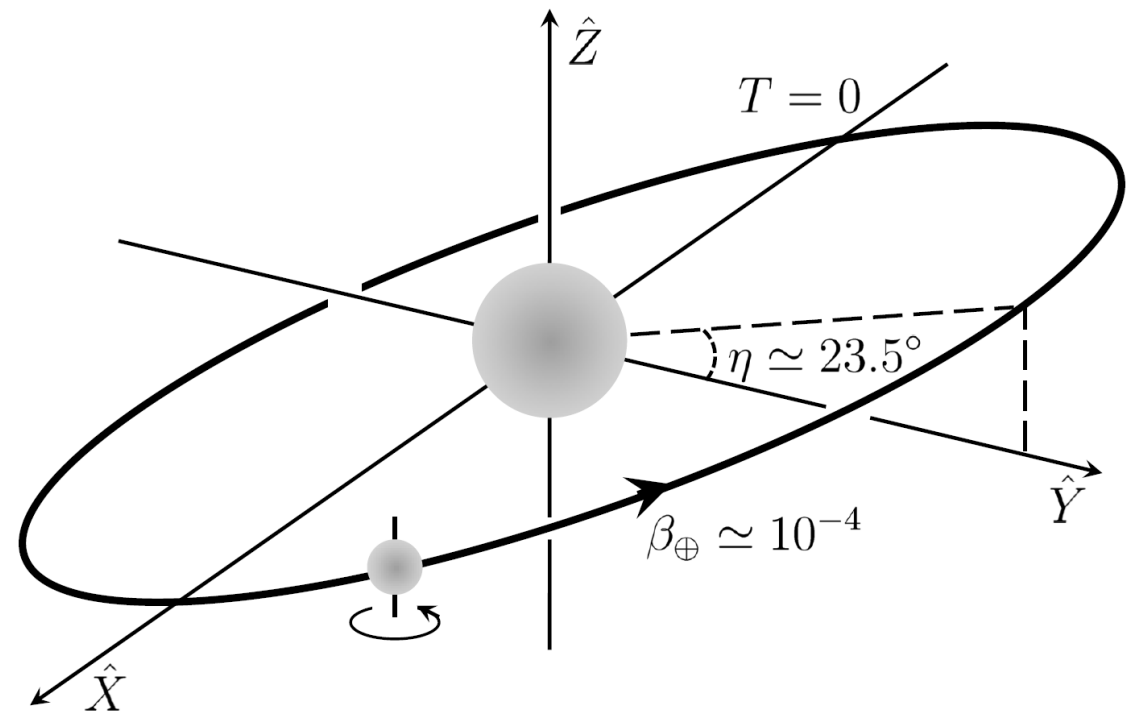

Figure 2.1: The canonical Sun-centered inertial reference frame of the SME.

The canonical Sun-centered reference frame is defined by a right-handed Cartesian coordinate system oriented so that the $Z$-axis points parallel to the Earth's axis of rotation. The $X-Y$ plane then corresponds to the Earth's equatorial plane. The $X$ axis points toward the vernal equinox and the origin of the time coordinate is chosen to coincide with the vernal equinox of the year 2000.

As the Earth rotates, the orientation of an Earth-based laboratory changes periodically relative to the Sun-centered reference frame. This period, called the "sidereal" period, is the time it takes for the Earth to complete one rotation relative to the "fixed" stars (the Sun-centered frame). Because the Earth is progressing along its orbit as it rotates, the resulting "sidereal day" is slightly shorter than one solar day (23 hours, 56 minutes, 4.091 seconds versus 24 hours). 
The relative velocity of the Earth about the Sun is around $30 \mathrm{~km} / \mathrm{s}$, corresponding to a Lorentz gamma factor of approximately $\gamma-1 \approx 10^{-9}$. The boost of an Earthbased experiment relative to the Sun-centered reference frame is too small to produce a measurable effect in a search for Lorentz violation in top quark events. Transformation between the reference frame of such an experiment and the Sun-centered frame will therefore only involve rotations.

\subsection{Some previous SME studies}

CPT and Lorentz violation (LV) have been studied in a number of sectors of the Standard Model including gravity, electromagnetic interactions, and several lepton and baryon flavors. Indirect limits have even been set on the Higgs sector [16] before the particle's official discovery. Many of these studies take advantage of the expected sidereal or annual variation in one or more observable phenomena. Although tight constraints have been set on the magnitude of $\mathrm{LV}$ in a variety of particle sectors, such constraints generally involve the light charged leptons and quarks. Because they only interact weakly, the neutrino sector can be particularly difficult to study. Due to the large masses of the charged members of the third generation, the $b, t$, and $\tau$ have only relatively recently become accessible to experiments searching for LV.

Looking for sidereal modulations in the event rate of an experiment gives an easily accessible handle on possible Lorentz violation. For example, this technique has been used to probe SME parameters in the neutrino sector. The MINOS experiment examined the rate at which neutrinos produced at an accelerator at Fermilab arrived at both the near detector [17] and the far detector [18]. This rate could be sensitive to 
various $a_{\mu^{-}}$and $c_{\mu \nu}$-type SME coefficients parametrizing CPT and Lorentz violation in the neutrino sector through their effect on neutrino flavor oscillation parameters. Because of the large baselines of neutrino experiments (typically hundreds of meters to hundreds of kilometers), these measurements are often very sensitive to SME coefficients. MINOS was able to set limits on various components of these parameters at levels tighter than $10^{-20}$ and $10^{-23}$, respectively. The IceCube experiment in Antarctica further improved some of these constraints by searching for a sidereal variation in the flux of atmospherically produced muon neutrinos [19].

Like the lighter quarks, $b$ quarks bind with other quarks immediately upon their production and are only observable as part of a hadron system. However, effects of CPT and Lorentz violation can still contribute to the properties of, for example, $B$ mesons. While it is difficult to directly access SME coefficients in these systems, some linear combinations can be directly measured. The BaBar experiment set new constraints on components of the effective SME coefficient $\left(\Delta a^{B}\right)_{\mu}$ governing CPT violation in neutral $B$ meson mixing. This coefficient describes the difference between the effects of LV on the valence $d$ and $b$ quarks of the $B^{0}$ meson, where $\left(\Delta a^{B^{0}}\right)_{\mu}=$ $r_{d}\left(a_{D}\right)_{\mu 11}-r_{b}\left(a_{D}\right)_{\mu 33}$ and the $r_{q}$ are due to quark-binding and renormalization effects. By searching for a sidereal variation in the mixing of $B^{0}$ and $\bar{B}^{0}$ mesons produced in $\Upsilon(4 S) \rightarrow B \bar{B}$ decays [20], the experiment constrained the terms $\Delta\left(a^{B^{0}}\right)_{X}, \Delta\left(a^{B^{0}}\right)_{Y}$ and $\Delta\left(a^{B^{0}}\right)_{T}-0.30 \Delta\left(a^{B^{0}}\right)_{Z}$ to be less than a few times $10^{-15}$. Observations of an anomalous like-sign dimuon charge asymmetry in the $B_{s}^{0}-\bar{B}_{s}^{0}$ system at the $\mathrm{D} \varnothing$ detector provided a similar constraint in the $B_{s}$ sector, where the magnitude of the coefficient $\left(\Delta a^{B_{s}}\right)_{T}$ was limited to $\lesssim 10^{-11}[21]$.

CPT and LV are not constrained to be the same for all particle species, so each 
sector of the SM must be tested. Because of its extremely large mass compared to all other SM particles, the top quark has remained out of reach until recently. The Tevatron and LHC particle colliders have reached energies and event rates high enough to provide the first probe of CPT and Lorentz violation in top quark events. Kostelecký and Berger have derived the expected contribution from SME terms to the cross section for pair production of $t$ and $\bar{t}$ quarks [22]. The next chapter summarizes this result and explores how it can be utilized to set the first limits on Lorentz violation in the top quark sector. 


\section{Chapter 3}

\section{Lorentz Violation in the \\ Top-Quark Sector}

We investigate Lorentz and CPT violation in the top quark sector by examining the expected and observed production of top and antitop quark pairs $(t \bar{t})$ within a collider experiment. The expectation for the $t \bar{t}$ event rate is calculated from the matrix

element describing the $t \bar{t}$ production and decay process, including appropriate terms from the SME [22]. This represents the probability amplitude for the process to occur for specified initial- and final-state momenta, and is derived from the appropriate Lagrange density. We compare this matrix element to the Standard Model expectation in order to estimate the unique contribution from Lorentz- and CPT-violating effects.

Fig. 3.1 illustrates the process by which a $t \bar{t}$ pair is produced from a protonantiproton collision and subsequently decays "semileptonically" to a final state including one charged lepton. The SME contributions to the Lagrange density listed in Eq. (2.9) affect the propagators of the $t, \bar{t}, b$, and $\bar{b}$ quarks, as well as the $t \rightarrow b W^{+}$ 


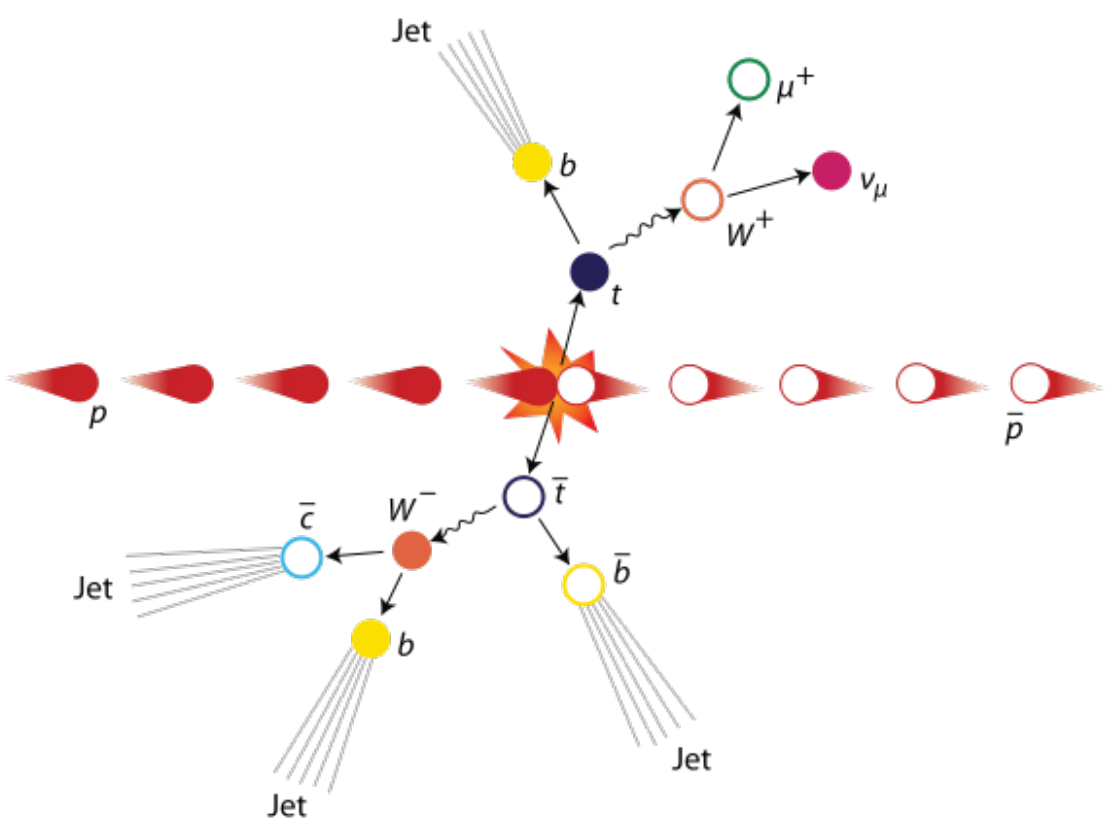

Figure 3.1: Illustration of the production and decay of a $t \bar{t}$ pair.

and $\bar{t} \rightarrow \bar{b} W^{-}$vertices. Although the left-handed $b$-quark field in the doublet $\left(Q_{3}\right)$ does enter this process at the $t$-b- $W$ vertices, the right-handed $b$-quark singlet field (equivalent to $D_{3}$ ) does not participate. Additionally, terms in this matrix element controlled by the $a_{\mu}$-type coefficients are suppressed at leading order [22]. While these coefficients controlling CPT-even terms in the Lagrange density do not produce observable effects at leading order in this $t \bar{t}$ process, this analysis is sensitive to several of the $c_{\mu \nu}$-type coefficients which couple to potentially observable effects from CPT-odd terms. The SME coefficients of interest to this analysis are therefore $\left(c_{Q}\right)_{\mu \nu 33}$ and $\left(c_{U}\right)_{\mu \nu 33}$.

For brevity, we define $\left(c_{L}\right)_{\mu \nu}=\left(c_{Q}\right)_{\mu \nu 33}$ and $\left(c_{R}\right)_{\mu \nu}=\left(c_{U}\right)_{\mu \nu 33}$. These matrices of coefficients are each symmetric and traceless. We also define the following linear combinations, in order to compare our results to those of SME studies in other particle 
sectors.

$$
\begin{aligned}
& c_{\mu \nu}=\left(c_{L}\right)_{\mu \nu}+\left(c_{R}\right)_{\mu \nu}, \\
& d_{\mu \nu}=\left(c_{L}\right)_{\mu \nu}-\left(c_{R}\right)_{\mu \nu} .
\end{aligned}
$$

\subsection{SME matrix element}

The phenomenological background of this analysis is based on the analytical work of V. A. Kostelecký and M. S. Berger of the Indiana University Center for Spacetime Symmetries (IUCSS). We provide the results of their derivation here.

At leading order in the SME coefficients, the matrix element describing the production and decay of a $t \bar{t}$ pair involves only the $\left(c_{L}\right)_{\mu \nu}$ and $\left(c_{R}\right)_{\mu \nu}$ coefficients. The squared matrix element for this process has the form

$$
|\mathcal{M}|^{2}=P F \bar{F}+\left(\delta P_{p}\right) F \bar{F}+\left(\delta P_{v}\right) F \bar{F}+P(\delta F) \bar{F}+P F(\delta \bar{F}) .
$$

The $P$ terms depend on the parton momenta at the $t \bar{t}$ vertex, while the $F$ and $\bar{F}$ terms are functions of the parton momenta in the $t$ and $\bar{t}$ decay vertices, respectively. The first term, $P F \bar{F}$, is the Lorentz-invariant contribution from the SM $t \bar{t}$ process. This receives corrections from the SME terms in the Lagrange density. These are associated with the $t$ and $\bar{t}$ propagators $\left(\delta P_{p}\right)$, the production vertex $\left(\delta P_{v}\right)$, and the $t$ and $\bar{t}$ decay vertices ( $\delta F$ and $\delta \bar{F}$, respectively). We list the full expression for each of these seven terms in the process $q \bar{q} \rightarrow t \bar{t} \rightarrow b W^{+} \bar{b} W^{-} \rightarrow b \bar{\ell} \nu b q^{\prime} \bar{q}^{\prime \prime}$ below.

In terms of the four-momenta of the various particles in the event, the components of the leading-order SM $t \bar{t}$ matrix element are 


$$
\begin{gathered}
P=\frac{g_{s}^{4}}{18 E^{4}}\left[\left(p_{q} \cdot p_{t}\right)\left(p_{\bar{q}} \cdot p_{\bar{t}}\right)+\left(p_{q} \cdot p_{\bar{t}}\right)\left(p_{\bar{q}} \cdot p_{t}\right)+\left(p_{q} \cdot p_{\bar{q}}\right) m_{t}^{2}\right], \\
F=-4 g_{W}^{4}\left[\frac{\left(p_{\nu} \cdot p_{b}\right)\left(p_{\bar{\ell}} \cdot p_{t}\right)}{\left(m_{t}^{2}-M_{t}^{2}\right)^{2}+\left(M_{t} \Gamma_{t}\right)^{2}}\right]\left[\frac{1}{\left(m_{\bar{\ell} \nu}^{2}-M_{W}^{2}\right)^{2}+\left(M_{W} \Gamma_{W}\right)^{2}}\right],
\end{gathered}
$$

and

$$
\bar{F}=-4 g_{W}^{4}\left[\frac{\left(p_{q^{\prime}} \cdot p_{\bar{b}}\right)\left(p_{\bar{q}^{\prime \prime}} \cdot p_{\bar{t}}\right)}{\left(m_{\bar{t}}^{2}-M_{t}^{2}\right)^{2}+\left(M_{t} \Gamma_{t}\right)^{2}}\right]\left[\frac{1}{\left(m_{q^{\prime} \bar{q}^{\prime \prime}}^{2}-M_{W}^{2}\right)^{2}+\left(M_{W} \Gamma_{W}\right)^{2}}\right]
$$

Here, $g_{W}$ is the weak coupling constant and $M_{t}, \Gamma_{t}, M_{W}$ and $\Gamma_{W}$ are the masses and widths of the top quark and $W$ boson. The quantities $m_{\bar{\ell} \nu}$ and $m_{q^{\prime} \bar{q}^{\prime \prime}}$ are the invariant masses of the lepton and neutrino from the $W^{+}$decay and the two jets from the $W^{-}$decay, respectively, and $m_{t}\left(m_{\bar{t}}\right)$ is the invariant mass of the three final state partons from the $t \rightarrow b W^{+}\left(\bar{t} \rightarrow \bar{b} W^{-}\right)$decay. The quantity $E$ is the energy of the initial-state quark or antiquark in the center-of-mass frame of the production vertex, corresponding to the Mandelstam variable $\hat{s}=4 E^{2}$.

The SME contribution from the $t$ and $\bar{t}$ propagators is

$$
\begin{aligned}
\delta P_{p}= & \frac{g_{s}^{4}}{18 E^{4}}\left(\left(c_{L}\right)_{\mu \nu}+\left(c_{R}\right)_{\mu \nu}\right)\left[\left(p_{q} \cdot p_{t}\right)\left(p_{\bar{t}}^{\mu} p_{\bar{q}}^{\nu}\right)+\left(p_{q} \cdot p_{\bar{t}}\right)\left(p_{t}^{\mu} p_{\bar{q}}^{\nu}\right)\right. \\
& \left.+\left(p_{\bar{q}} \cdot p_{t}\right)\left(p_{\bar{t}}^{\mu} p_{q}^{\nu}\right)+\left(p_{\bar{q}} \cdot p_{\bar{t}}\right)\left(p_{t}^{\mu} p_{q}^{\nu}\right)\right],
\end{aligned}
$$

and the contribution from the production vertex is 


$$
\begin{aligned}
\delta P_{v}= & \frac{g_{s}^{4}}{18 E^{4}}\left(\left(c_{L}\right)_{\mu \nu}+\left(c_{R}\right)_{\mu \nu}\right)\left[-\left(p_{q} \cdot p_{\bar{q}}\right)\left(p_{t}^{\mu} p_{\bar{t}}^{\nu}+p_{\bar{t}}^{\mu} p_{t}^{\nu}\right)\right. \\
& -\left(p_{t} \cdot p_{\bar{t}}+m_{t}^{2}\right)\left(p_{q}^{\mu} p_{\bar{q}}^{\nu}+p_{\bar{q}}^{\mu} p_{q}^{\nu}\right) \\
& +\left(p_{q} \cdot p_{t}\right) p_{\bar{q}}^{\mu} p_{\bar{t}}^{\nu}+\left(p_{q} \cdot p_{\bar{t}}\right) p_{\bar{q}}^{\mu} p_{t}^{\nu} \\
& \left.+\left(p_{\bar{q}} \cdot p_{t}\right) p_{q}^{\mu} p_{\bar{t}}^{\nu}+\left(p_{\bar{q}} \cdot p_{\bar{t}}\right) p_{q}^{\mu} p_{t}^{\nu}\right] .
\end{aligned}
$$

The SME contributions at the $t \rightarrow b W^{+}$and $\bar{t} \rightarrow \bar{b} W^{-}$vertices are

$$
\begin{aligned}
\delta F=2 g_{W}^{4} & {\left[\frac{1}{\left(m_{t}^{2}-M_{t}^{2}\right)^{2}+\left(M_{t} \Gamma_{t}\right)^{2}}\right]\left[\frac{1}{\left(m_{\bar{\ell} \nu}^{2}-M_{W}^{2}\right)^{2}+\left(M_{W} \Gamma_{W}\right)^{2}}\right]\left(c_{L}\right)_{\mu \nu} } \\
\times & {\left[\left(p_{b} \cdot p_{t}\right)\left(p_{\nu}^{\mu} p_{\bar{\ell}}^{\nu}+p_{\bar{\ell}}^{\mu} p_{\nu}^{\nu}\right)+\left(p_{b} \cdot p_{\nu}\right)\left(p_{t}^{\mu} p_{\bar{\ell}}^{\nu}+p_{\bar{\ell}}^{\mu} p_{t}^{\nu}\right)\right.} \\
& -\left(p_{b} \cdot p_{\bar{\ell}}\right)\left(p_{t}^{\mu} p_{\nu}^{\nu}+p_{\nu}^{\mu} p_{t}^{\nu}\right)-\left(p_{t} \cdot p_{\nu}\right)\left(p_{b}^{\mu} p_{\bar{\ell}}^{\nu}+p_{\bar{\ell}}^{\mu} p_{b}^{\nu}\right) \\
& \left.+\left(p_{t} \cdot p_{\bar{\ell}}\right)\left(p_{b}^{\mu} p_{\nu}^{\nu}+p_{\nu}^{\mu} p_{b}^{\nu}\right)+\left(p_{\nu} \cdot p_{\bar{\ell}}\right)\left(p_{b}^{\mu} p_{t}^{\nu}+p_{t}^{\mu} p_{b}^{\nu}\right)\right]
\end{aligned}
$$

and

$$
\begin{aligned}
& \delta \bar{F}=2 g_{W}^{4} {\left[\frac{1}{\left(m_{\bar{t}}^{2}-M_{t}^{2}\right)^{2}+\left(M_{t} \Gamma_{t}\right)^{2}}\right]\left[\frac{1}{\left(m_{q^{\prime} \bar{q}^{\prime \prime}}^{2}-M_{W}^{2}\right)^{2}+\left(M_{W} \Gamma_{W}\right)^{2}}\right]\left(c_{L}\right)_{\mu \nu} } \\
& \times\left[\left(p_{\bar{t}} \cdot p_{\bar{b}}\right)\left(p_{q^{\prime}}^{\mu} p_{\bar{q}^{\prime \prime}}^{\nu}+p_{\bar{q}^{\prime}}^{\mu} p_{q^{\prime}}^{\nu}\right)+\left(p_{\bar{t}} \cdot p_{q^{\prime}}\right)\left(p_{\bar{b}}^{\mu} p_{\bar{q}^{\prime \prime}}^{\nu}+p_{\bar{q}^{\prime}}^{\mu} p_{\bar{b}}^{\nu}\right)\right. \\
& \quad-\left(p_{\bar{t}} \cdot p_{\bar{q}^{\prime \prime}}\right)\left(p_{\bar{b}}^{\mu} p_{q^{\prime}}^{\nu}+p_{q^{\prime}}^{\mu} p_{\bar{b}}^{\nu}\right)-\left(p_{\bar{b}} \cdot p_{q^{\prime}}\right)\left(p_{\bar{t}}^{\mu} p_{\bar{q}^{\prime \prime}}^{\nu}+p_{\bar{q}^{\prime}}^{\mu} p_{\bar{t}}^{\nu}\right) \\
&\left.\quad+\left(p_{\bar{b}} \cdot p_{\bar{q}^{\prime \prime}}\right)\left(p_{\bar{t}}^{\mu} p_{q^{\prime}}^{\nu}+p_{q^{\prime}}^{\mu} p_{\bar{t}}^{\nu}\right)+\left(p_{q^{\prime}} \cdot p_{\bar{q}^{\prime \prime}}\right)\left(p_{\bar{t}}^{\mu} p_{\bar{b}}^{\nu}+p_{\bar{b}}^{\mu} p_{\bar{t}}^{\nu}\right)\right]
\end{aligned}
$$

where $q^{\prime}$ is a down-type quark and $\bar{q}^{\prime \prime}$ is an up-type antiquark. The other possible semileptonic decay mode $t \bar{t} \rightarrow b W^{+} \bar{b} W^{-} \rightarrow b q^{\prime} \bar{q}^{\prime \prime} \bar{b} \ell \bar{\nu}$ is related to these expressions by the replacements $\nu \rightarrow q^{\prime}, \bar{\ell} \rightarrow \bar{q}^{\prime \prime}, q^{\prime} \rightarrow \ell$, and $\bar{q}^{\prime \prime} \rightarrow \bar{\nu}$.

It is important to note that the production corrections $\delta P_{p}$ and $\delta P_{v}$ depend on both 
$\left(c_{L}\right)_{\mu \nu}$ and $\left(c_{R}\right)_{\mu \nu}$, while the decay corrections $\delta F$ and $\delta \bar{F}$ only depend on $\left(c_{L}\right)_{\mu \nu}$. This will effect the sensitivity of our analysis to the different coefficients. Furthermore, for $t \bar{t}$ pairs produced in an Earth-based laboratory, production probabilities for various parton four-momenta configurations will depend on the orientation of the laboratory frame relative to the Sun-centered reference frame. The coefficients $\left(c_{L}\right)_{\mu \nu}$ and $\left(c_{R}\right)_{\mu \nu}$ are defined in the Sun-centered reference frame, and the relative orientation of the two frames will vary as the Earth rotates. This effect will introduce a time dependence in the SME contribution to the $t \bar{t}$ production and decay process.

Finally, we also note that the matrix element of Eq. (3.2) represents the production process $q \bar{q} \rightarrow t \bar{t}$, but $t \bar{t}$ pair production can also proceed through gluon fusion $(g g \rightarrow$ $t \bar{t})$. However, in $p \bar{p}$ collisions at the Tevatron the latter production mode contributes only about $15 \%$ to the total event rate and is therefore suppressed. We expect this matrix element to suffice for the first measurement of Lorentz violation in the top quark sector at the $\mathrm{D} \varnothing$ experiment. We will comment further on its applicability to experiments at the LHC during the discussion of the expectations for this analysis at the ATLAS detector.

\subsection{Time-dependent cross section}

To derive the observational effects of this matrix element, we consider the expression for the total effective cross section for $t \bar{t}$ production and semileptonic decay. This quantity represents the probability that a $t \bar{t}$ pair is produced in the collision interaction, decays to a semileptonic final state, and is accepted, detected, and reconstructed by the experiment. The total cross section $\sigma(t)$ observed in the detector is 


$$
\sigma(t)=\int d x \epsilon(x) \mathcal{B} \int d y \frac{d \sigma_{\mathrm{SME}}}{d y}(y, t) P(x \mid y)
$$

$\mathcal{B}$ is the branching fraction of $t \bar{t}$ into final states containing a single lepton $(e$ or $\mu)$. $x$ reflects the observed kinematic variables, i.e. the four-momenta of the measured jets and leptons, while $P(x \mid y)$ describes the probability of the partonic final state $y$ to be measured as $x$ in the detector. $\epsilon(x)$ is the efficiency (acceptance, trigger, and reconstruction) to accept an event specified by $x$. $d \sigma_{\mathrm{SME}} / d y$ contains the SME parameters we wish to determine and is dependent on time through the changing orientation of the detector frame relative to the canonical Sun-centered reference frame.

We expand the expression for the differential cross section, including SME terms, by comparing it to the SM differential cross section $d \sigma_{\mathrm{SM}} / d y$.

$$
\frac{d \sigma_{\mathrm{SME}}}{d y}=\frac{d \sigma_{\mathrm{SME}} / d y}{d \sigma_{\mathrm{SM}} / d y} \frac{d \sigma_{\mathrm{SM}}}{d y}
$$

This ratio of differential cross sections is equivalent to the ratio of the squared matrix element with SME corrections $\left(|\mathcal{M}|_{\text {SME }}^{2}\right)$ to the squared matrix element without those corrections $\left(|\mathcal{M}|_{\text {SM }}^{2}\right)$. We can therefore define an event weight $w(y, t)$ which collects the SME contribution to the $t \bar{t}$ process as a function of parton momentum and time.

$$
w(y, t)=\frac{d \sigma_{\mathrm{SME}} / d y}{d \sigma_{\mathrm{SM}} / d y}=\frac{|\mathcal{M}|_{\mathrm{SME}}^{2}}{|\mathcal{M}|_{\mathrm{SM}}^{2}} .
$$

Inserting this expression into Eq. (3.10), we find

$$
\sigma(t)=\int d x \epsilon(x) \mathcal{B} \int d y w(y, t) \frac{d \sigma_{\mathrm{SM}}}{d y} P(x \mid y)
$$


where $w(y, t)$ can be expanded as

$$
\begin{aligned}
w(y, t) & =\frac{1}{P F \bar{F}}\left(P F \bar{F}+\left(\delta P_{p}+\delta P_{v}\right) F \bar{F}+P(\delta F) \bar{F}+P F(\delta \bar{F})\right) \\
& =1+\left(\left(c_{L}\right)_{\mu \nu}+\left(c_{R}\right)_{\mu \nu}\right)\left(\frac{\delta P_{p}^{\mu \nu}}{P}+\frac{\delta P_{v}^{\mu \nu}}{P}\right)+\left(c_{L}\right)_{\mu \nu}\left(\frac{\delta F^{\mu \nu}}{F}+\frac{\delta \bar{F}^{\mu \nu}}{\bar{F}}\right)
\end{aligned}
$$

We define $\delta P_{p}^{\mu \nu}, \delta P_{v}^{\mu \nu}, \delta F^{\mu \nu}$, and $\delta \bar{F}^{\mu \nu}$ as in Equations (3.6-3.9) prior to contraction with the SME coefficients $\left(c_{L}\right)_{\mu \nu}$ and $\left(c_{R}\right)_{\mu \nu}$.

\subsubsection{Monte Carlo approximation}

The integrals in Eq. (3.13) can be approximated using a set of simulated (Monte Carlo) events generated via Standard Model semileptonic $t \bar{t}$ production and reconstructed using a full detector simulation. Events are generated with PYTHIA [23] and the subsequent final state particles in each event are propagated through a simulation of the detector in order to model the detector response. We can discretize the parton phase space $y$ into $n_{y}$ bins such that

$$
\begin{aligned}
\sigma(t) & \approx \int d x \epsilon(x) \sum_{i}^{n_{y}} w\left(y_{i}, t\right) P\left(x \mid y_{i}\right) \mathcal{B} \int_{y_{i}} d y \frac{d \sigma_{\mathrm{SM}}}{d y} \\
& \approx \int d x \epsilon(x) \sum_{i}^{n_{y}} w\left(y_{i}, t\right) P\left(x \mid y_{i}\right) \mathcal{B} \Delta \sigma_{i}^{\mathrm{SM}} .
\end{aligned}
$$

Here, $y_{i}$ is the set of four-momenta at the center of phase-space bin $i$, and $\Delta \sigma_{i}^{\mathrm{SM}}$ is the contribution to the total SM cross section attributed to that bin of phase space. $\Delta \sigma_{i}^{\mathrm{SM}}$ can be related to the number of Monte Carlo events generated in $i\left(n_{i}\right)$, the total number of Monte Carlo events generated over the entire phase space of $y\left(n_{\mathrm{MC}}\right)$, and the resulting total Standard Model cross section for $t \bar{t}$ production $\left(\sigma_{\mathrm{SM}}\right)$ : 


$$
\Delta \sigma_{i}^{\mathrm{SM}}=n_{i} \frac{\sigma_{\mathrm{SM}}}{n_{\mathrm{MC}}}
$$

Using this relationship,

$$
\sigma(t) \approx \frac{\sigma_{\mathrm{SM}}}{n_{\mathrm{MC}}} \mathcal{B} \int d x \epsilon(x) \sum_{i}^{n_{y}} w\left(y_{i}, t\right) P\left(x \mid y_{i}\right) n_{i} .
$$

In the limit that the number of bins $n_{i}$ approaches infinity, the occupancy of any bin $i$ becomes either 0 events or 1 event. The expression for the cross section then simplifies to

$$
\sigma(t) \approx \frac{\sigma_{\mathrm{SM}}}{n_{\mathrm{MC}}} \mathcal{B} \int d x \epsilon(x) \sum_{j \in \mathrm{MC}}^{n_{\mathrm{MC}}} w\left(y_{j}, t\right) P\left(x \mid y_{j}\right),
$$

with the summation now over the number of generated Monte Carlo events.

To model the detector response $P(x \mid y)$, the detector simulation transforms each set of generator level parton momenta $y$ into a set of final state observables $x$, randomly selecting one of several possible $x$ for each $y$. This smearing across several observable final states is small, and we can treat $P(x \mid y)$ as approximately one-to-one. This implies

$$
\sigma(t) \approx \frac{\sigma_{\mathrm{SM}}}{n_{\mathrm{MC}}} \mathcal{B} \sum_{j \in \mathrm{MC}}^{n_{\mathrm{MC}}} \epsilon\left(x_{j}\right) w\left(y_{j}, t\right) .
$$

We can now substitute Eq. (3.14) into Eq. (3.18). Expanding the sum, we find 


$$
\begin{aligned}
\sigma(t) \approx \frac{\sigma_{\mathrm{SM}}}{n_{\mathrm{MC}}} \mathcal{B} \sum_{j \in \mathrm{MC}}^{n_{\mathrm{MC}}} \epsilon\left(x_{j}\right)[1 & +\left(\left(c_{L}\right)_{\mu \nu}+\left(c_{R}\right)_{\mu \nu}\right)\left(\frac{\delta P_{p}\left(y_{j}, t\right)}{P\left(y_{j}\right)}+\frac{\delta P_{v}\left(y_{j}, t\right)}{P\left(y_{j}\right)}\right) \\
& \left.+\left(c_{L}\right)_{\mu \nu}\left(\frac{\delta F^{\mu \nu}\left(y_{j}, t\right)}{F\left(y_{j}\right)}+\frac{\delta \bar{F}^{\mu \nu}\left(y_{j}, t\right)}{\bar{F}\left(y_{j}\right)}\right)\right] .
\end{aligned}
$$

The detector efficiency (acceptance, trigger, reconstruction, etc.) $\epsilon\left(x_{j}\right)=1$ if the simulated event $x_{j}$ is accepted by the trigger and reconstruction algorithms, while $\epsilon\left(x_{j}\right)=0$ if it is rejected. The summation in Eq. (3.20) then runs over the number of accepted Monte Carlo events $\left(n_{\text {Acc }}\right)$ and can be expressed as

$$
\begin{aligned}
\sum_{k \in \text { Acc }}^{n_{\mathrm{Acc}}}[1 & +\left(\left(c_{L}\right)_{\mu \nu}+\left(c_{R}\right)_{\mu \nu}\right)\left(\frac{\delta P_{p}\left(y_{k}, t\right)}{P\left(y_{k}\right)}+\frac{\delta P_{v}\left(y_{k}, t\right)}{P\left(y_{k}\right)}\right) \\
& \left.+\left(c_{L}\right)_{\mu \nu}\left(\frac{\delta F^{\mu \nu}\left(y_{k}, t\right)}{F\left(y_{k}\right)}+\frac{\delta \bar{F}^{\mu \nu}\left(y_{k}, t\right)}{\bar{F}\left(y_{k}\right)}\right)\right] .
\end{aligned}
$$

We define matrices $A_{P}^{\mu \nu}$ and $A_{F}^{\mu \nu}$ corresponding to the averages of the appropriate SME contributions as

$$
\begin{aligned}
& A_{P}^{\mu \nu}=\frac{1}{n_{\mathrm{Acc}}} \sum_{k \in \mathrm{Acc}}^{n_{\mathrm{Acc}}}\left(\frac{\delta P_{p}^{\mu \nu}\left(y_{k}\right)}{P\left(y_{k}\right)}+\frac{\delta P_{v}^{\mu \nu}\left(y_{k}\right)}{P\left(y_{k}\right)}\right), \\
& A_{F}^{\mu \nu}=\frac{1}{n_{\mathrm{Acc}}} \sum_{k \in \mathrm{Acc}}^{n_{\mathrm{Acc}}}\left(\frac{\delta F^{\mu \nu}\left(y_{k}\right)}{F\left(y_{k}\right)}+\frac{\delta \bar{F}^{\mu \nu}\left(y_{k}\right)}{\bar{F}\left(y_{k}\right)}\right) .
\end{aligned}
$$

Using these definitions to simplify Eq. (3.20), we find

$$
\sigma(t) \approx \sigma_{\mathrm{SM}} \mathcal{B} \frac{n_{\mathrm{Acc}}}{n_{\mathrm{MC}}}\left[1+\left(\left(c_{L}\right)_{\mu \nu}+\left(c_{R}\right)_{\mu \nu}\right) A_{P}^{\mu \nu}+\left(c_{L}\right)_{\mu \nu} A_{F}^{\mu \nu}\right]
$$

The quantity $n_{\mathrm{Acc}} / n_{\mathrm{MC}}$ represents the total efficiency $\epsilon$ (the combined fiducial, trigger, and reconstruction efficiencies) to accept and reconstruct a semileptonic $t \bar{t}$ event in 
the detector. The factor $\sigma_{\mathrm{SM}} \mathcal{B} \epsilon$ is the product of the SM cross section, the branching fraction to the $\ell+$ jets final states, and the overall efficiency.

The matrices $A_{P}^{\mu \nu}$ and $A_{F}^{\mu \nu}$ are the averages of the kinematic components of the four $\delta$ terms in the ratio $|\mathcal{M}|_{\text {SME }}^{2} / P F \bar{F}$, evaluated in the Sun-centered reference frame. We wish to evaluate them in the reference frame of the experiment, and so introduce the matrix $R_{\alpha}^{\mu}(t)$ to transform these terms from the detector frame to the Sun-centered frame. The final, full expression for the time dependent $t \bar{t}$ cross section into semileptonic final states, including Lorentz violating contributions from the SME in the top quark sector, is

$$
\sigma(t) \approx \sigma_{\mathrm{SM}} \mathcal{B} \epsilon\left[1+\left(\left(c_{L}\right)_{\mu \nu}+\left(c_{R}\right)_{\mu \nu}\right) R_{\alpha}^{\mu}(t) R_{\beta}^{\nu}(t) A_{P}^{\alpha \beta}+\left(c_{L}\right)_{\mu \nu} R_{\alpha}^{\mu}(t) R_{\beta}^{\nu}(t) A_{F}^{\alpha \beta}\right]
$$

For brevity we collect the SME contribution to Eq. (3.25) into the function $f_{\mathrm{SME}}(t)$.

$$
\sigma(t) \approx \sigma_{\mathrm{SM}} \mathcal{B} \epsilon\left(1+f_{\mathrm{SME}}(t)\right)
$$

\section{A note on $\mathrm{LO}$ vs $\mathrm{NLO}$}

While the Monte Carlo data set used for this analysis was produced using next-to-

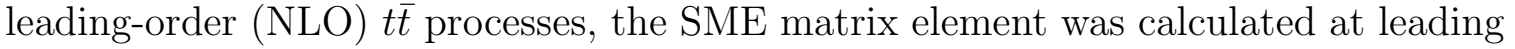
order (LO). However, if we assume that the NLO corrections to the LO events are the same for calculations including SME terms, we find that the LO SME matrix element is sufficient to calculate event weights for the NLO SM Monte Carlo events.

Let $p$ be the set of four-momenta in the event describing the contributions at leading order, and $q$ be the four-momenta in the event describing the NLO contributions 
(i.e. the extra particles in the higher-order diagrams beyond the fundamental participants in the $t \bar{t}$ production and decay process). Define a k-factor $k_{\mathrm{NLO}}(p, q)$ such that

$$
\frac{d \sigma_{\mathrm{NLO}}}{d p d q}=k_{\mathrm{NLO}}(p, q) \frac{d \sigma_{\mathrm{LO}}}{d p} .
$$

We assume that $k_{\mathrm{NLO}}^{\mathrm{SME}}(p, q) \approx k_{\mathrm{NLO}}^{\mathrm{SM}}(p, q) \equiv k(p, q)$. As the size of the SME coefficients decreases, this approximation becomes more exact. In the case of small SME coefficients we expect the difference to be negligible.

This assumption yields

$$
w_{\mathrm{NLO}}(p, q, t) \equiv \frac{d \sigma_{\mathrm{NLO}}^{\mathrm{SME}} / d p d q}{d \sigma_{\mathrm{NLO}}^{\mathrm{SM}} / d p d q}=\frac{k(p, q)\left(d \sigma_{\mathrm{LO}}^{\mathrm{SME}} / d p\right)}{k(p, q)\left(d \sigma_{\mathrm{LO}}^{\mathrm{SM}} / d p\right)} \equiv w_{\mathrm{LO}}(p, t) .
$$

We can therefore determine the expected $t \bar{t}$ cross section by averaging the weights $w_{\mathrm{LO}}$ calculated for each event in a set of SM $t \bar{t}$ events produced with a next-to-leadingorder Monte Carlo generator.

\subsection{Experimental signature}

It is clear from Eq. (3.25) that the Lorentz-violating contributions from the SME add terms which couple to the orientation and boost of the Earth-based experimental reference frame relative to the canonical Sun-centered reference frame. The relative velocity of the Earth as it revolves about the Sun is small (about $10^{-4} \mathrm{c}$ ), and the associated boost effect is one part in $10^{-9}$. This implies that the sensitivities of the corresponding SME coefficients will be suppressed. There remains, however, a 
potentially sizable contribution from the relative orientation of the experiment frame to the Sun-centered frame. This orientation changes as the Earth rotates, introducing a periodic time dependence of one sidereal day (Fig. 3.2).

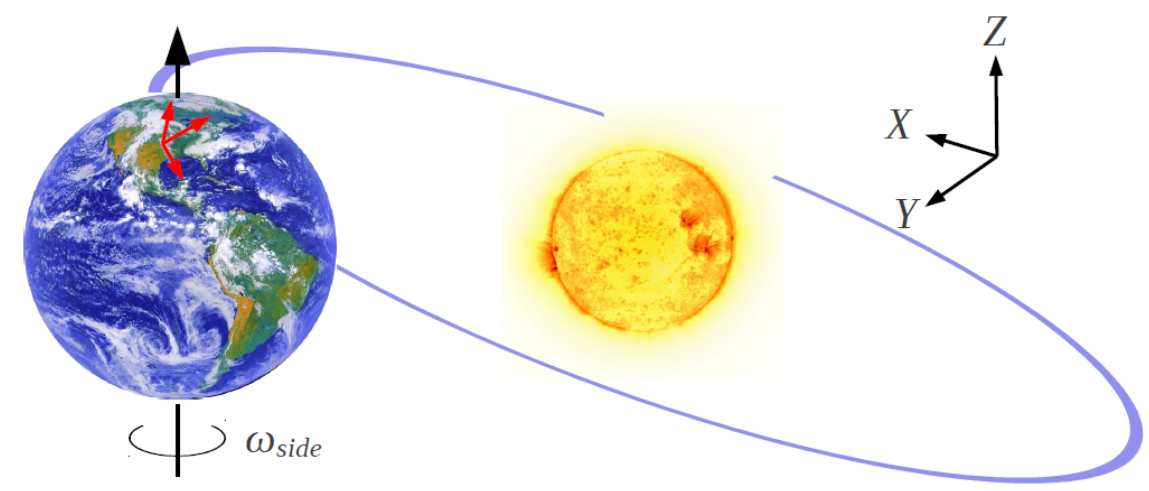

Figure 3.2: Illustration of the relationship between the reference frame of an experiment and the Sun-centered reference frame. (Earth and Sun image credits: NASA)

We expect that any observable effects of Lorentz violation in the top quark sector would manifest as a sidereal time dependence in the $t \bar{t}$ event rate. We search for such signs of LV in the top sector, as parametrized by the SME, using $t \bar{t}$ events produced by the Tevatron proton-antiproton collider and detected by the $\mathrm{D} \emptyset$ experiment. The following chapter provides an overview of these facilities. Chapter five discusses the methods utilized by the $\mathrm{D} \emptyset$ experiment to select candidate $t \bar{t}$ events and estimate the purity of such samples. Chapter six discusses the expected effect on the $t \bar{t}$ event rate due to the time dependent cross section described by Eq. (3.25), and presents the results of our analysis. 


\section{Chapter 4}

\section{The DØ Experiment}

The partnership between the $\mathrm{D} \varnothing$ experiment and the Fermilab Tevatron collider has facilitated the exploration of frontier particle physics and the expansion of our understanding of the fundamental constituents of the universe. The Tevatron [24] delivered collisions between protons $(p)$ and antiprotons $(\bar{p})$ with a center-of-mass energy of $1.96 \mathrm{TeV}$ until its shutdown in September of 2011. Located at one of the points along the Tevatron synchrotron ring where beams of proton and antiproton intersect, the $\mathrm{D} \emptyset$ detector [25] collected data about the final-state particles resulting from these ppbar collisions. With the end of the Tevatron's operations the $\mathrm{D} \emptyset$ detector is being prepared for decommissioning and dismantling, although analysis of data collected by the experiment continues.

In $t \bar{t}$ production at this $p \bar{p}$ collision energy, about $85 \%$ of the time a quark from the proton interacts with an antiquark from the antiproton, while the rest of the time the fundamental interaction is between two gluons [26]. From this data, members of the $\mathrm{D} \emptyset$ collaboration reconstruct and measure the properties of the initial, inter- 
mediate, and final-state particles participating in the collision events. The detector was designed to provide sensitivity to a wide range of Standard Model processes and event topologies, making it a tool which provides a model-independent probe of particle interactions. This chapter seeks to provide a basic overview of the accelerator and detector. More detailed information can be found in references [24]-[25].

The $\mathrm{D} \emptyset$ detector consists of many layers of sensitive radiation detectors, organized into subdetectors that each specialize in measuring properties of specific types of particles. A central tracking system provides measurements used to reconstruct the trajectories of charged particles and the positions of event vertices. The tracking detectors are located within a magnetic field oriented parallel to the collision axis, bending the trajectories of charged particles to provide a measurement of their momenta. After traversing the central tracking volume, particles from the collision event reach a sophisticated liquid-argon/uranium calorimeter system. Particles such as electrons and photons are absorbed by electromagnetic calorimeters, providing a measurement of their kinetic energy. The energy of strongly-interacting particles, such as protons and neutrons, is absorbed by both the electromagnetic calorimetry and further layers of hadronic calorimeters. Because muons deposit only a small fraction of their energy as they travel through the calorimeters, additional layers of charged particle tracking detectors comprise the outer-most layers of the $\mathrm{D} \emptyset$ detector. Iron toroidal magnets are embedded in this muon spectrometer system to provide an additional momentum measurement. Finally, the only signature of weakly-interacting neutrinos is an imbalance in the reconstructed transverse momentum of the event.

Numerous discoveries and precision measurements have been made at the D $\varnothing$ experiment over its lifetime. During the initial run from 1992-1999 (Run I), the 
experiment produced perhaps its most famous result - the joint discovery, with the CDF collaboration, of the top quark [11]. Since then, the DØ collaboration has made accurate measurements of many of its properties [27]. Precision measurements of many other components of the Standard Model have also been made, including the mass of the $\mathrm{W}$ bosons [28], gauge boson coupling strengths [29], and mixing of $B_{s}$ and $\bar{B}_{s}$ mesons [30]. The experiment has placed many new limits on new physics beyond the Standard Model such as supersymmetry [31], leptoquarks [32], and extra dimensions [33]. D $\varnothing$, together with the CDF experiment, also helped narrow the window in which the elusive Higgs boson may reside [34].

Between 2000 and 2001, the Tevatron accelerator facility was upgraded to improve the delivered luminosity by a factor of almost 10. During this time, several upgrades were made to the $\mathrm{D} \emptyset$ detector. The data taking period from 2001 to 2011 is referred to as Run II. This chapter provides a brief overview of the Tevatron accelerator and a detailed description of the upgraded $\mathrm{D} \varnothing$ detector used to gather data during this period.

\subsection{The Fermilab Tevatron collider}

The Tevatron collider was a synchrotron used to accelerate and collide counterrotating beams of protons and antiprotons. The collider was the final component of a multistage accelerator facility (Fig. 4.1) designed to generate both protons and antiprotons, accelerate them to high energies, and provide beams to several different experiments.

The chain which shepherds protons toward collisions begins at a Cockcroft-Walton 


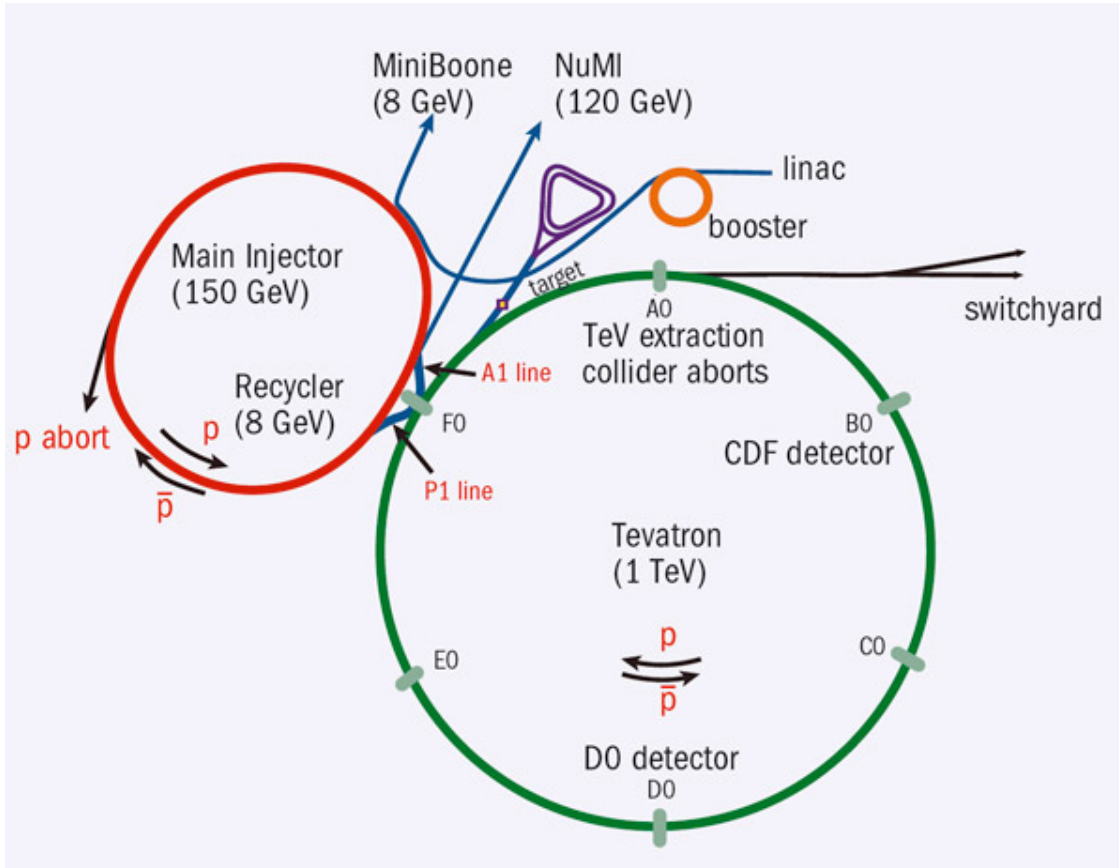

Figure 4.1: The Fermilab accelerator complex.

generator, where hydrogen gas is ionized into $H^{-}$ions that are pre-accelerated to 750 $\mathrm{keV}$. The negative ions then enter a linear accelerator (Linac) consisting of a series of $\mathrm{RF}$ accelerating cavities which increase the beam energy to $400 \mathrm{MeV}$. The electrons are then stripped from the $H^{-}$ions by a carbon foil, leaving the protons to pass into the first synchrotron stage. The Booster, a small synchrotron $474 \mathrm{~m}$ in circumference, further accelerates the beam to an energy of $8 \mathrm{GeV}$.

From the Booster, the proton beam travels to the Main Injector (MI). At $3.3 \mathrm{~km}$ in circumference, this synchrotron plays several important roles in Fermilab operations. The MI accumulates several proton bunches from the Booster into one high-intensity bunch and accelerating it to $150 \mathrm{GeV}$ for, tasks which included injection into the Tevatron. The MI also accelerates proton bunches to $120 \mathrm{GeV}$, some of which are 
directed at a fixed nickel target where a variety of secondary particles are produced, including antiprotons. For $p \bar{p}$ collisions in the Tevatron, these antiprotons were accumulated and fed back into the MI for acceleration before injection into the final accelerator stage. Uncollided antiprotons from the Tevatron were re-injected into the MI for deceleration before traveling to the Recycler, installed in the MI tunnel in 2005 to increase the Tevatron's luminosity performance. These antiprotons were stored in the Recycler and later combined with additional antiproton bunches from the antiproton source before returning to the MI for acceleration and injection into the Tevatron.

The final synchrotron stage was the Tevatron. Proton and antiproton beams counter-circulated through a $6.3 \mathrm{~km}$ ring of 774 niobium-titanium (NbTi) superconducting dipole magnets and $216 \mathrm{NbTi}$ superconducting quadrupole magnets, cooled with liquid helium maintained at a temperature of approximately $4 \mathrm{~K}$. Interlaced with these steering and focusing magnets, RF cavities accelerated the beams to 0.98 $\mathrm{TeV}$ each. Each beam consisted of 36 bunches of particles, with close to $10^{11}$ protons and $10^{10}$ antiprotons in each bunch. Bunches from the proton and antiproton beams intersected every $396 \mathrm{~ns}$ at two locations along the circumference of the Tevatron. At the peak operating luminosity, an average of about 12 inelastic $p \bar{p}$ interactions occurred in each bunch crossing with a center-of-mass energy of up to $\sqrt{s}=1.96$ $\mathrm{TeV}$. Two multipurpose particle detectors, the $\mathrm{D} \varnothing$ and CDF detectors, were located at these interaction regions and studied the products of these high-energy collision events. 


\subsection{The $\mathrm{D} \emptyset$ detector}

Named simply for the intersection point at which it is located on the Tevatron ring, the $\mathrm{D} \varnothing$ detector is a complex and sophisticated multipurpose particle physics experiment designed to record the products of $p \bar{p}$ collisions delivered by the accelerator. The detector surrounds the interaction region with cylindrical symmetry along the beamline and provides nearly complete coverage in solid-angle. Fig. 4.2 provides an overview of the detector layout through a three-quarter cutaway perspective. Fig. 4.3 shows a detailed view of the detector in cross section, with the central tracking system, calorimeters, and muon spectrometer labeled. Proton-antiproton interactions were distributed as a 20 cm-wide Gaussian distribution along the $z$-axis with a typical maximum transverse size of a few tens of micrometers, occurring within a beryllium beam pipe of approximately $19 \mathrm{~mm}$ in radius.

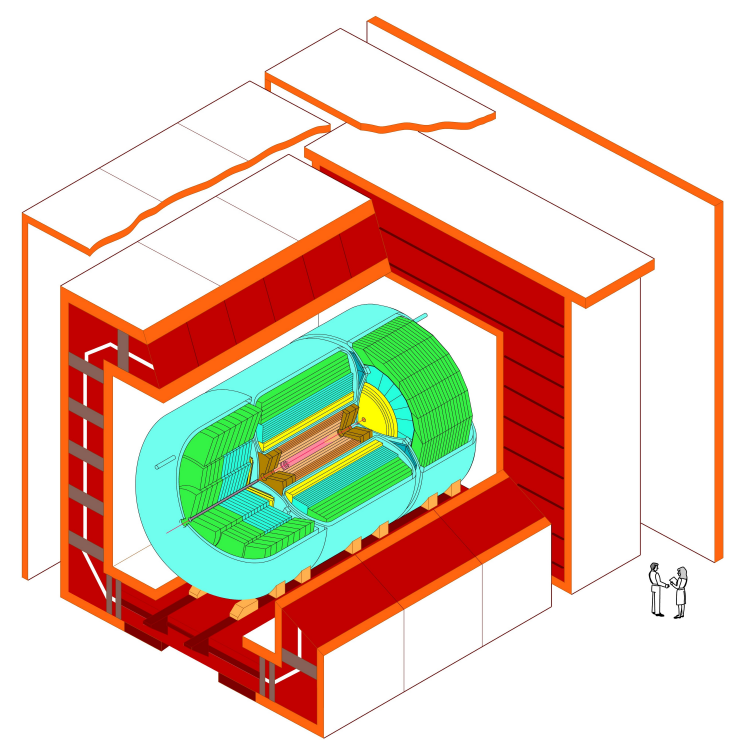

Figure 4.2: The D $\varnothing$ detector. A pair of physicists in their natural environment illustrates scale. 


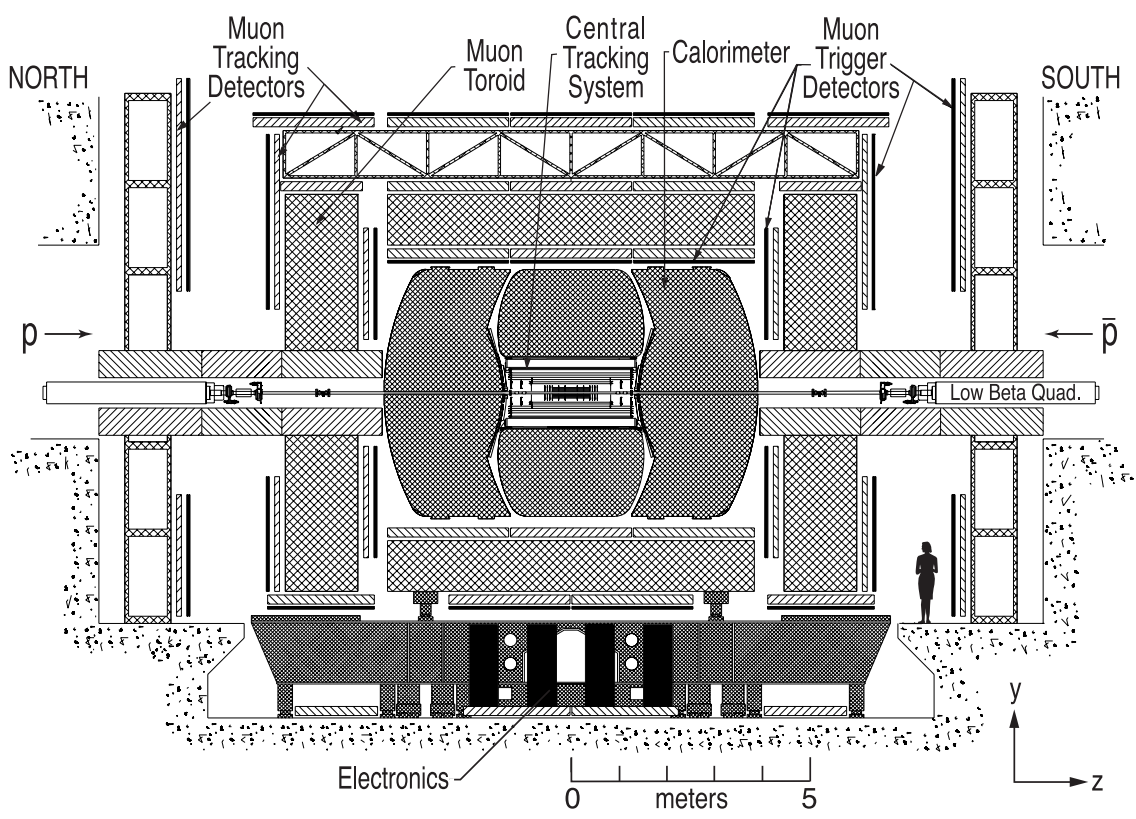

Figure 4.3: Cross section of the D $\varnothing$ detector in $x-z$.

The $\mathrm{D} \varnothing$ detector employs a right-handed coordinate system with the $z$-axis oriented along the proton beam direction. The $y$-axis is upward, and the $x$-axis points away from the center of the accelerator. The azimuthal and polar angles $\phi$ and $\theta$ are measured relative to the $x$ - and $z$-axis, respectively, while the $r$ coordinate denotes the perpendicular radial distance from the $z$-axis. The pseudorapidity, $\eta=-\ln [\tan (\theta / 2)]$, is a good approximation to the true rapidity $y=1 / 2 \ln \left[\left(E+p_{z} c\right) /\left(E-p_{z} c\right)\right]$ in the practical limit $\left(m c^{2} / E\right) \rightarrow 0$. Regions of large $|\eta|$ are referred to as "forward" regions in the detector.

\subsubsection{Central tracking system}

At the innermost volume of the $\mathrm{D} \varnothing$ detector, two sophisticated subdetectors provide spatial position data along the paths of charged particles produced in the interaction 
region. The Silicon Microstrip Tracker (SMT) and the Central Fiber Tracker (CFT) are situated within the $2 \mathrm{~T}$ field of a superconducting solenoidal magnet oriented along the detector's z-axis (Fig. 4.4). Charged particles experience a bending force due to this magnetic field, curving their paths into helical trajectories. The radius of curvature depends on the strength of the magnetic field and the transverse component of the particle's momentum relative to the field, while the direction of curvature is determined by the sign of the charge. Reconstructed tracks of charged particles can be extrapolated forward to the calorimeters and backward to the interaction point. The central tracking system therefore serves two vital functions for event reconstruction: 1) reconstructing tracks of charged particles and 2) reconstructing vertices from the ensemble of tracks in the event.

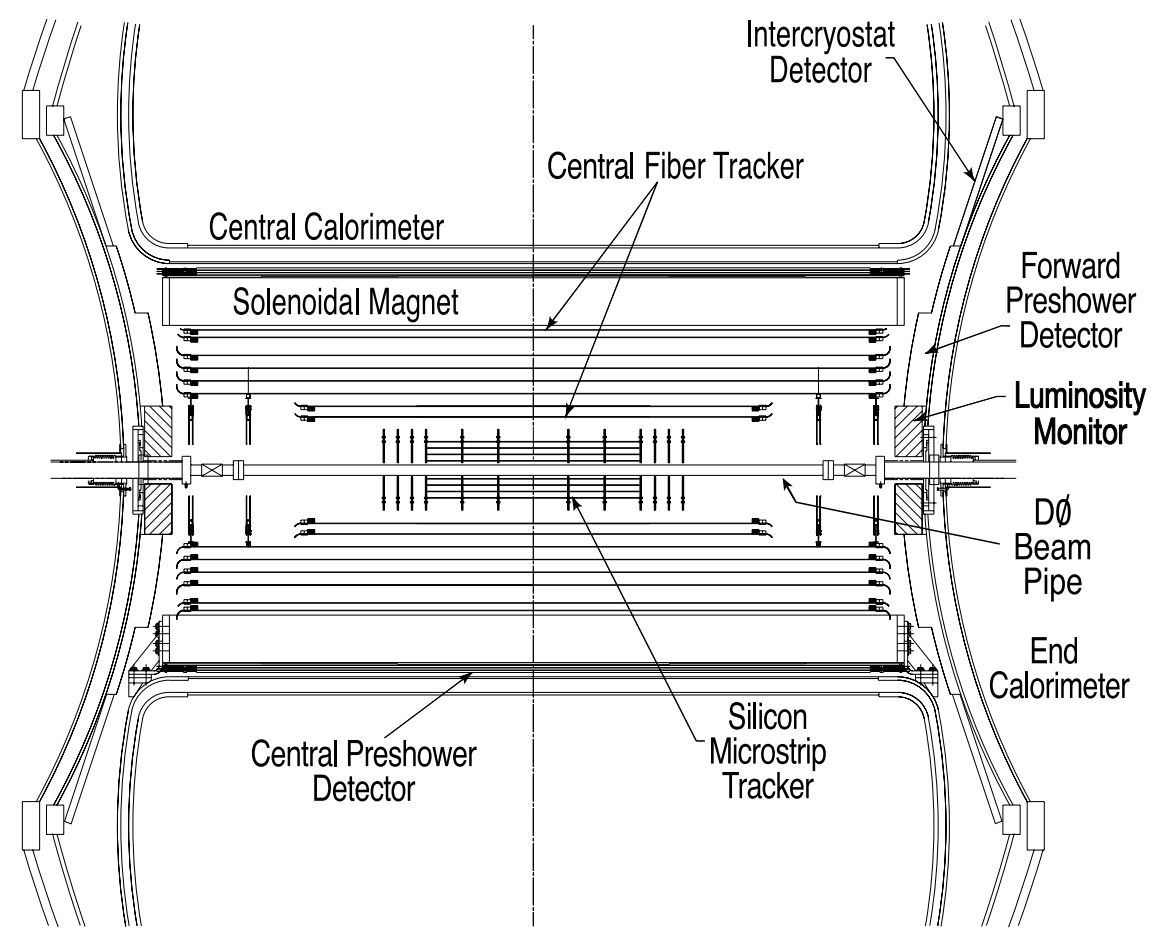

Figure 4.4: The DØ central tracking system. 
The SMT and CFT together provide accurate track momentum and vertex position resolution. Tracks can be reconstructed up to pseudorapidities $|\eta|<2.5$, and primary interaction vertices can be resolved with an accuracy of about $35 \mu \mathrm{m}$ in the transverse plane. The high impact parameter resolution can resolve secondary vertices resulting from the decay of hadrons containing $b$-quarks, providing a method of tagging jets of particles originating from $b$-quarks. Combined transverse momentum resolution $\delta p_{T} / p_{T}$ in the central region ranges from $2 \%$ for tracks with $p_{T}=1 \mathrm{GeV}$ to $11 \%$ for tracks with $p_{T}=100 \mathrm{GeV}$.

The SMT is a finely segmented tracking detector which surrounds the beryllium beam pipe at the core of the detector. The central region of the SMT consists of barrel modules oriented along the beam pipe, providing $r-\phi$ measurements, interspersed with disk modules to provide both $r-z$ and $r-\phi$ measurements, while assemblies of disk modules occupy the forward regions. Together the SMT's barrel and disk modules are comprised of about 800,000 individual silicon sensors and provide a position resolution of about $20 \mu \mathrm{m}$.

The CFT provides additional tracking in the region 20 to $52 \mathrm{~cm}$ from the beam pipe. It consists of layers of scintillating fibers, alternating between orientations parallel to the beam axis and at a stereo angle of \pm 3 degrees relative to that axis. The fiber material produces a flash of light when ionized by a passing charged particle. This flash of light is transmitted along the fiber to solid-state photon counters. Signals from successive layers are combined to construct spatial position measurement, with each doublet layer providing a resolution of about $100 \mu \mathrm{m}$. 


\subsubsection{Calorimetry}

The $\mathrm{D} \varnothing$ calorimeter systems are divided into three parts - the central calorimeter (CC) section covering $|\eta|$ up to about 1.1 and two endcap calorimeters (EC) that cover regions up to $|\eta| \approx 4$.2. Jets can be reconstructed in the central calorimeter with relative transverse energy resolution around 10\%. The calorimeters use liquid argon as the active medium, and each is housed in a separate cryostat to maintain the detector at a temperature of approximately $90 \mathrm{~K}$. Preshower detectors sandwiched between the calorimeter cryostats and the central solenoidal magnet provide additional measurements for both tracking and calorimetry, and scintillators positioned between the $\mathrm{CC}$ and $\mathrm{EC}$ cryostats provide additional measurements of developing calorimeter showers within the overlap region.

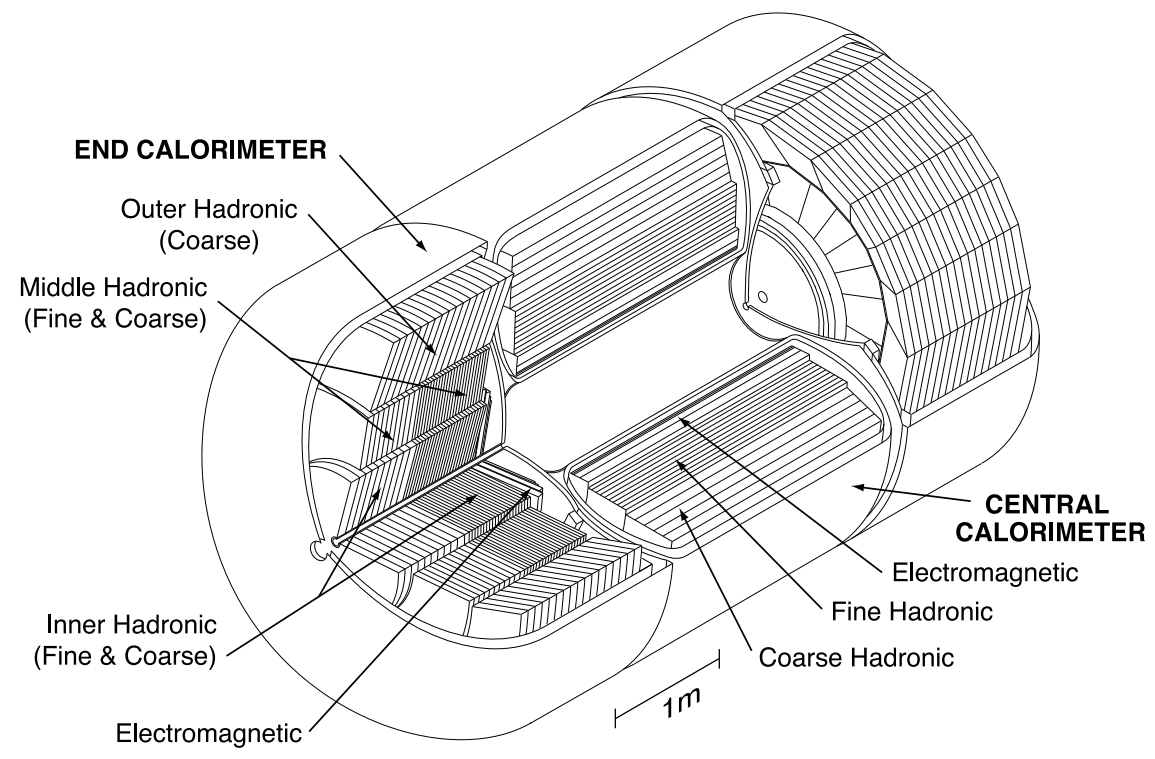

Figure 4.5: The D $\varnothing$ calorimeters.

Each calorimeter is divided into layers of electromagnetic calorimetry and hadronic 
calorimetry of varying granularity (Fig. 4.5). The electromagnetic (EM) calorimeter modules use thin plates of depleted uranium for absorber plates. The absorber plates of the finely segmented hadronic sections are made from a uranium-niobium $(2 \%)$ alloy, while the coarse hadronic sections contain thick plates of copper (in the CC) or stainless steel (in the EC).

\subsubsection{Muon spectrometer}

The characteristic energies of muons produced in collision events at the Tevatron make them minimally ionizing particles (MIP), meaning they loose only a tiny fraction of their energy through interactions with detector materials and pass through the calorimeter volume unabsorbed. The $\mathrm{D} \emptyset$ detector therefore operates with an additional tracking volume outside of the calorimeter systems. This muon spectrometer consists of two subdetector types - proportional drift tubes and scintillation trigger counters. Figs. 4.6 and 4.7 show exploded views of the distribution of these two subdetector systems, respectively. $10 \mathrm{~cm}$-diameter proportional drift tubes (PDT) cover the region $|\eta|<1$, and smaller $1 \mathrm{~cm}$ mini drift tubes (MDT) cover the forward regions $1<|\eta|<2$. The scintillation trigger counters provide additional muon position data, as well as information for triggering and to reduce the cosmic-ray background. A 1.8 $\mathrm{T}$ toroidal magnet system lies between the A- and B-layers of the muon spectrometer system to provide an additional measurement of muon momentum. 


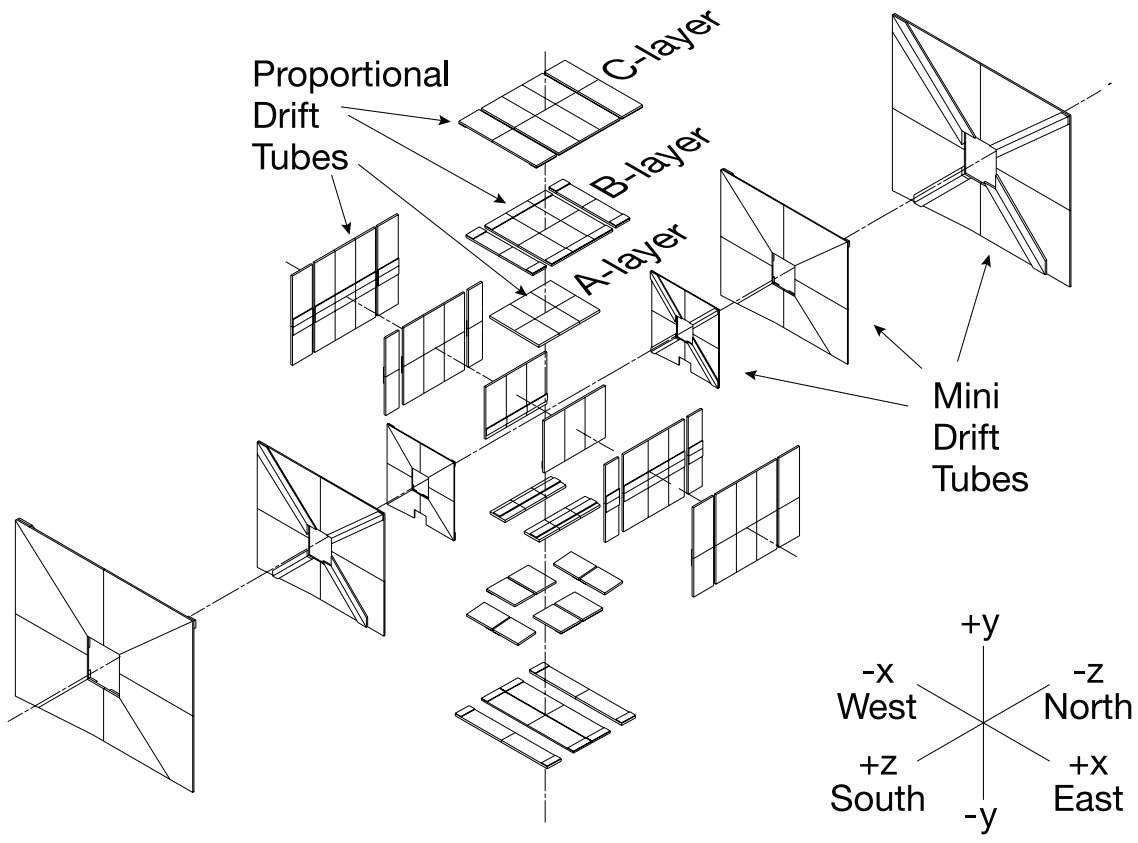

Figure 4.6: Exploded view of the PDT and MDT detectors.

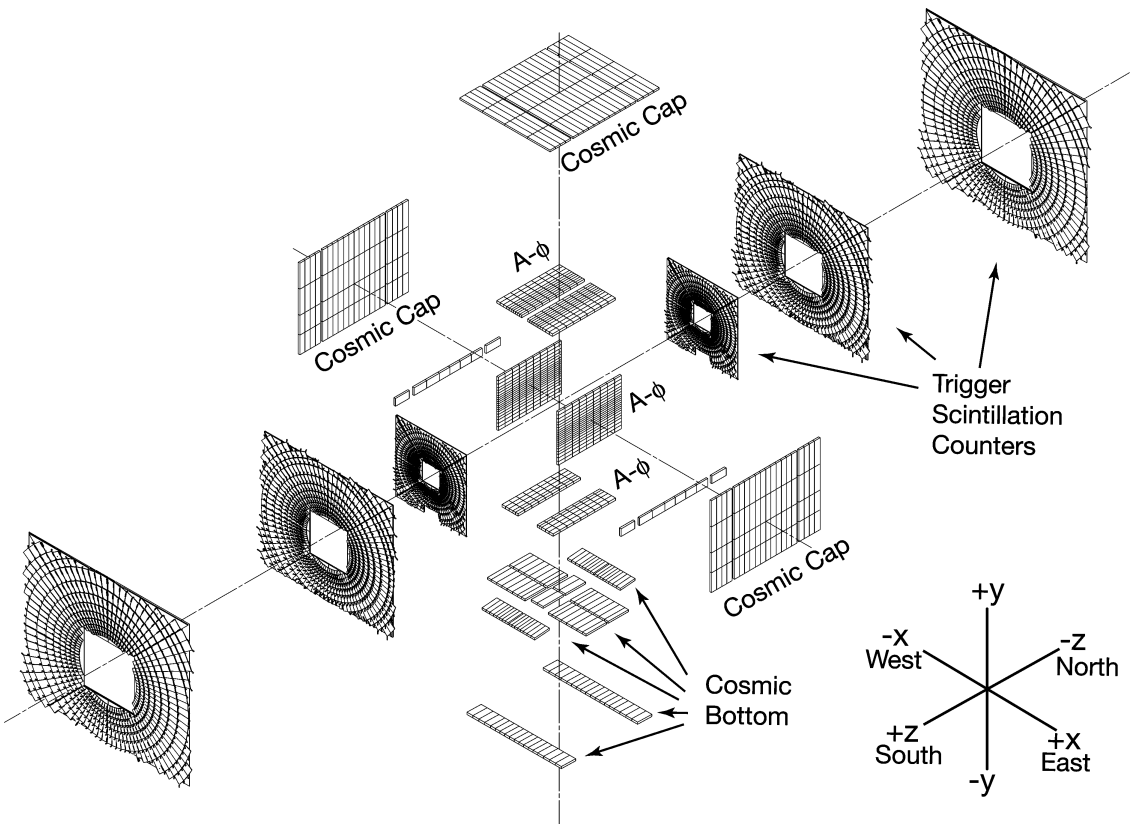

Figure 4.7: Exploded view of the scintillation trigger counters. 


\subsubsection{Luminosity monitor}

Instantaneous luminosity is determined using inelastic $p \bar{p}$ collisions monitored by a dedicated detector. The luminosity monitor (LM) consists of two discs of segmented scintillation counters located at $z= \pm 140 \mathrm{~cm}$, between the SMT and endcap calorimeters (Fig. 4.8). The luminosity is determined by estimating the average number of inelastic collisions per beam crossing [35]. The fundamental unit of time over which luminosity is measured is the luminosity block (LB). Each LB is indexed by a unique luminosity block number (LBN), incremented after $60 \mathrm{~s}$ or sooner by request from the trigger or detector operation systems.

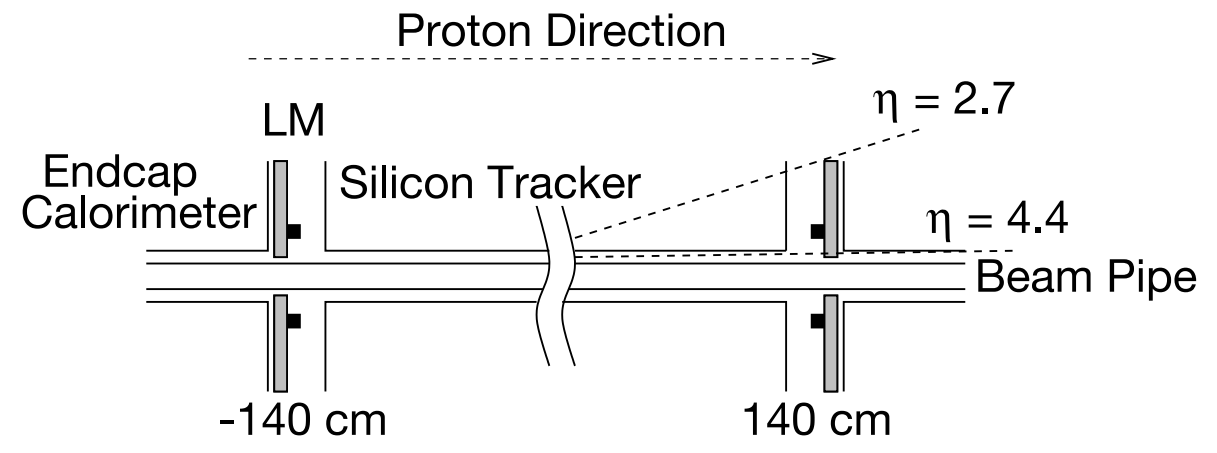

Figure 4.8: The DØ luminosity monitor.

\subsubsection{Trigger}

Collision events are delivered at a rate of about $1.7 \mathrm{MHz}$, but only those few events with topologies consistent with scientifically interesting events are desired. The DØ detector employs a three-level trigger system to monitor collision events and select only those which pass specific acceptance requirements to be recorded for analysis. Fig. 4.9 shows a schematic overview of the trigger and data acquisition systems. 
Events are selected by the Level 1 trigger based on coarse preliminary output from the tracking, calorimeter, and muon systems using a combination of hardware, firmware, and custom-built CPUs, passing events to the Level 2 trigger at a reduced rate of approximately $2 \mathrm{kHz}$. The Level 2 trigger also employs a combination of hardware and firmware to further filter events, based on event topology examined at a finer detector granularity and including correlations across different subdetectors. Finally, events are passed from the Level 2 trigger at a rate of about $1 \mathrm{kHz}$ to the Level 3 trigger. These events are analyzed on a computing farm, where software algorithms designed to perform a fast reconstruction of the entire event accept those with potential scientific interest at a rate of $\approx 100 \mathrm{~Hz}$. These selected events are then recorded for detailed analysis.

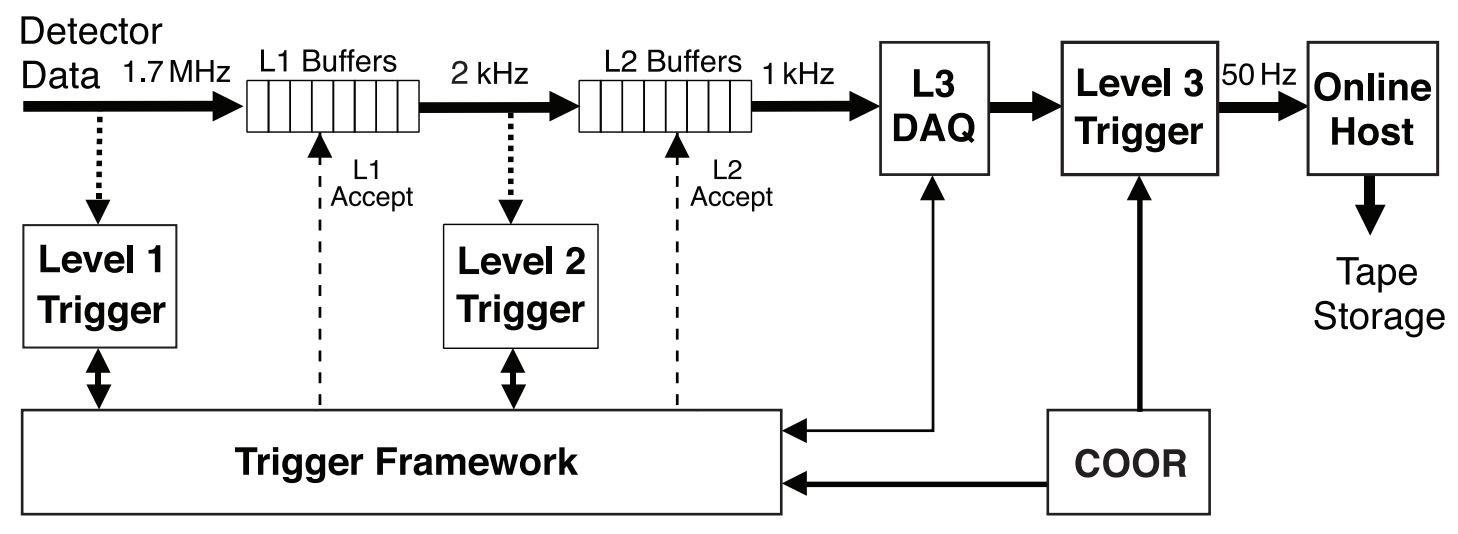

Figure 4.9: Overview of the D $\varnothing$ trigger and data acquisition systems. 


\section{Chapter 5}

\section{Top Quark Event Selection at DØ}

To search for the signature of Lorentz violation in the top quark sector we extend the methods of the $t \bar{t}$ cross section measurement performed by the $\mathrm{D} \emptyset$ experiment using data from Run II of the Fermilab Tevatron Collider [36]. This measurement was made using events decaying via the semileptonic mode $\left(t \bar{t} \rightarrow \ell \nu b q \bar{q}^{\prime} \bar{b}\right.$, where $\left.\ell=e, \mu\right)$. We extend this analysis to search for signs of LV in this process by examining the selected candidate $t \bar{t}$ events as a function of sidereal time. The cross section analysis is divided into separate measurements using events with final states consisting of either a single electron accompanied by jets ("e+jets") or a single muon accompanied by jets (" $\mu+$ jets"). Reference [36] presents a detailed discussion of the cross section

measurement. In this chapter we present a brief summary of the methods used by that analysis to select the events containing $t \bar{t}$ pair candidates chosen for our study. 


\subsection{Data set}

The cross section measurement was performed using data collected with the DØ detector during Run II of the Tevatron collider, corresponding to a total integrated luminosity of $5.3 \pm 0.3 \mathrm{fb}^{-1}$. During 2006, partway through Run II, extensive upgrades were made to the $\mathrm{D} \varnothing$ detector [25]. The period before this upgrade is referred to as Run IIa and the period afterward is Run IIb. Approximately $1 \mathrm{fb}^{-1}$ of integrated luminosity was collected and analyzed during Run IIa, yielding a value for the inclusive $t \bar{t}$ cross section $\sigma_{t \bar{t}}$ of $7.62 \pm 0.85 \mathrm{pb}[37]$. The data set was subsequently extended by an additional $\approx 4.3 \mathrm{fb}^{-1}$ of integrated luminosity during Run IIb. Using the data from both Run IIa and Run IIb, the experiment refined the value for $\sigma_{t \bar{t}}$ to $7.78_{-0.64}^{+0.77}$ pb, in agreement with the predictions of the Standard Model [36].

Trigger conditions were similar between the two periods. The primary trigger requirement for the $e+$ jets selection was the presence of either an electron or, in the case of a low- $p_{T}$ electron, an electron accompanied by a jet. During Run IIa, events were accepted into the $\mu+$ jets selection when the final state included a muon and a jet. This latter requirement was relaxed during Run IIb, only requiring a single muon. The full list of the applied trigger algorithms is reproduced from Ref. [38] in Appendix A.

\subsection{Event selection}

Events recorded after the trigger selections undergo full reconstruction. Object-level requirements refine the event selection to exclude events incompatible with a $t \bar{t}$-like topology while maintaining a low signal rejection. The primary vertex (PV) is re- 
quired to lie within $60 \mathrm{~cm}$ of the detector center along the $z$-axis, guaranteeing the majority of tracks fall within the SMT acceptance. Reconstructed electrons, muons, and jets are selected with requirements on their transverse momentum and pseudorapidity. The missing transverse energy provides a measurement of the final-state neutrino.

Jets are reconstructed using the "Run II cone" algorithm [39], where association of neighboring calorimeter deposits is optimized to identify jets with a benchmark radius $\mathcal{R}=\sqrt{\Delta \eta^{2}+\Delta \phi^{2}}=0.5$. From the set of all identified jets in the reconstructed event, only those with transverse momentum $p_{T}>20 \mathrm{GeV}$ and pseudorapidity $|\eta|<2.5$ are considered. Candidate $t \bar{t}$ events must contain more than one jet satisfying these requirements, and the leading jet is additionally required to be reconstructed with $p_{T}>40 \mathrm{GeV}$.

Collisions delivered during Run IIb occurred with higher instantaneous luminosity and typically contained multiple proton-antiproton interactions within the same bunch crossing. To reject jets coming from these additional $p \bar{p}$ interactions, jets selected for consideration as part of the $t \bar{t}$ final state in events from Run IIb are required to contain at least 3 tracks from the PV.

Candidate events in the semileptonic decay modes must also contain a single isolated lepton to pass acceptance. For the $e+$ jets sample, the event must contain only one isolated electron with $p_{T}>20 \mathrm{GeV}$ and $|\eta|<1.1$ (the fiducial coverage of the central calorimeter section). To be included in the $\mu+$ jets sample, there must be only one isolated muon with $p_{T}>20 \mathrm{GeV}$ and $|\eta|<2.0$. Events containing more than one isolated lepton of either flavor with $p_{T}>15 \mathrm{GeV}$ are rejected.

Finally, the associated neutrino contributes to the missing transverse energy $\mathbb{E}_{T}$ 
of the event, with the requirements $\not_{T}>20 \mathrm{GeV}$ for the electron channel and $\mathbb{E}_{T}>$ $25 \mathrm{GeV}$ for the muon channel.

The jets reconstructed in events containing a $t \bar{t}$ pair include those coming from the underlying $b$ quarks. These jets often display key features that can be used to differentiate them from jets evolved from lighter quarks (coming from the hadronically decaying $W$ boson) or from e.g. final-state gluon radiation. One such feature is the presence of a secondary vertex displaced from the PV within the jet cone, owing to the relatively long lifetime of the $B$ mesons formed during hadronization of the $b$ quark. A neural network $(\mathrm{NN})$ is trained to discriminate between $b$-jets and jets from other quark flavors by combining a variety of input variables describing the secondary vertex and its relationship to the PV and the rest of the tracks in the event [40]. The "medium" $b$-tag working point was chosen, giving a single $b$-tag probability of around 0.46 .

Because the event selection requirements and associated efficiencies are different for $e+$ jets events versus $\mu+$ jets events, these two channels are analyzed separately. The data is also divided between Run IIa and Run IIb. Due to differences in background contributions discussed below, the data is further divided according to jet multiplicity ( 2 jets, 3 jets, or $>3$ jets). Finally, it is useful to split the data into additional subsamples according to the number of jets tagged as $b$-jet candidates $(0$, 1 , or $>1)$.

The full cross section analysis [36] distinguishes between events with "tight" lepton requirements used for signal extraction and "loose" lepton requirements which are dominated by background. To minimize contributions from background (non- $t \bar{t}$ ) events while retaining maximum statistics, we choose events with the "tight" lepton 
selection requirements and exactly $1 b$-tagged jet. Tables 5.1 and 5.2 list the number of events in each jet multiplicity which will be examined to search for a sidereal time dependence resulting from violation of Lorentz invariance in the $t \bar{t}$ event.

Table 5.1: Number of $e+$ jets $t \bar{t}$ candidates in the "tight" lepton selection with exactly $1 b$-tagged jet.

\begin{tabular}{lccc}
\hline \hline & 2 jets & 3 jets & $>3$ jets \\
\hline Run IIa & 453 & 198 & 112 \\
Run IIb & 1590 & 648 & 289 \\
\hline Total & 2048 & 846 & 401 \\
\hline \hline
\end{tabular}

Table 5.2: Number of $\mu+$ jets $t \bar{t}$ candidates in the "tight" lepton selection with exactly $1 b$-tagged jet.

\begin{tabular}{lccc}
\hline \hline & 2 jets & 3 jets & $>3$ jets \\
\hline Run IIa & 317 & 140 & 109 \\
Run IIb & 1139 & 426 & 236 \\
\hline Total & 1456 & 566 & 345 \\
\hline \hline
\end{tabular}

\subsection{Sample composition}

A variety of physics processes can produce reconstructed event topologies that make them indistinguishable from true $t \bar{t}$ events. This results in a dilution of the signal content in the selected event samples. The sensitivity to LV effects coupling to the actual $t \bar{t}$ events is subsequently reduced. In order to properly account for these background contributions, we require an estimate of the composition of each of our event selections in terms of the signal and various backgrounds. 
By far the largest physics background is from production of a $W$ boson and associated jets. These $W+$ jets events dominate the background contribution to $t \bar{t}$ events, but other processes also contribute at a non-negligible level. Events consisting of a $Z$ boson and associated jets can contribute when, for example, the $Z$ decays to two leptons but only one is reconstructed within the detector. A tiny fraction of the very large number of multijet events from basic QCD processes will contain a jet which deposits most of its energy in the electromagnetic portion of the calorimeter, thereby emulating the expected $t \bar{t}$ topology. Small contributions also come from diboson events ( $W W, W Z$, and $Z Z$ ) and single top quark production. Reference [36] estimates the contribution from each of these sources of background using a variety of Monte Carlo and data-based methods. We collect the relevant yields for our chosen $t \bar{t}$ event selections in Tables 5.3 and 5.4 as a function of the number of jets in the event, and Fig. 5.1 illustrates the combined yields versus jet multiplicity.

Events from these various background processes are more likely to contain fewer jets than true $t \bar{t}$ events. Because of the low background content in the $e+>3$-jets and $\mu+>3$-jets samples, we choose these for our investigation of violation of Lorentz invariance. The $\ell+2$-jets and $\ell+3$-jets samples consist mostly of background (principally $W+$ jets) events and provide a useful cross-check. 
Table 5.3: Comparison of yields for $e+n$-jets channels for data in the $1 b$-tag selection to contributions expected from $t \bar{t}$ signal and sources of background. Uncertainties include statistical and systematic contributions.

\begin{tabular}{crcc}
\hline \hline$n$-jets & \multicolumn{1}{c}{$n=2$} & $n=3$ & \multicolumn{1}{c}{$n>3$} \\
\hline Sample & \multicolumn{3}{c}{ Yield } \\
\hline$W+$ jets & $1360 \pm 90$ & $316 \pm 26$ & $55 \pm 10$ \\
Multijet & $197 \pm 25$ & $75 \pm 8$ & $23 \pm 3$ \\
$Z+$ jets & $68 \pm 15$ & $26 \pm 6$ & $6 \pm 2$ \\
Other & $148 \pm 19$ & $41 \pm 6$ & $8 \pm 1$ \\
$t \bar{t}$ & $265 \pm 22$ & $381 \pm 30$ & $322 \pm 31$ \\
\hline Total & $2038 \pm 97$ & $839 \pm 37$ & $413 \pm 25$ \\
Observed & 2043 & 846 & 401 \\
\hline \hline
\end{tabular}

Table 5.4: Comparison of yields for $\mu+n$-jets channels for data in the $1 b$-tag selection to contributions expected from $t \bar{t}$ signal and sources of background. Uncertainties include statistical and systematic contributions.

\begin{tabular}{crcc}
\hline \hline$n$-jets & \multicolumn{1}{c}{$n=2$} & $n=3$ & \multicolumn{1}{c}{$n>3$} \\
\hline Sample & \multicolumn{3}{c}{ Yield } \\
\hline$W+$ jets & $1081 \pm 69$ & $261 \pm 20$ & $63 \pm 8$ \\
Multijet & $38 \pm 24$ & $14 \pm 5$ & $6 \pm 2$ \\
$Z+$ jets & $68 \pm 15$ & $19 \pm 5$ & $4 \pm 1$ \\
Other & $118 \pm 15$ & $32 \pm 4$ & $7 \pm 1$ \\
$t \bar{t}$ & $163 \pm 14$ & $262 \pm 21$ & $240 \pm 22$ \\
\hline Total & $1468 \pm 77$ & $589 \pm 28$ & $318 \pm 17$ \\
Observed & 1456 & 566 & 345 \\
\hline \hline
\end{tabular}




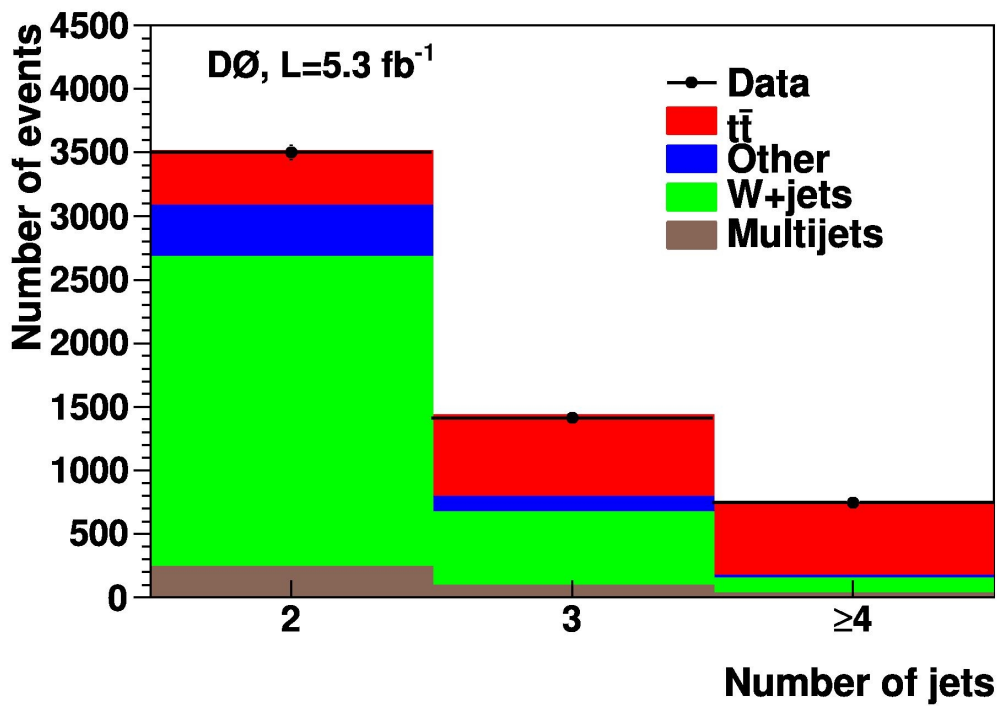

Figure 5.1: Jet multiplicity distribution for $\ell+$ jets events with $1 b$-tagged jet for backgrounds and contributions from $t \bar{t}$ signal ( $e+$ jets and $\mu+$ jets combined) [36]. 


\subsection{Event times}

In order to estimate any dependence on sidereal time in the $t \bar{t}$ event rate, our analysis requires the time of production of each event selected as a $t \bar{t}$ candidate by the criteria outlined above. Times are recorded by the D $\varnothing$ trigger and data acquisition system as the number of continuous seconds elapsed since the 00:00:00 coordinated universal time (UTC) of January 1, 1970. The fundamental unit of time over which luminosity is collected is the luminosity block (LB), corresponding to up to $60 \mathrm{~s}$ of delivered collisions. We identify the beginning and end of the LB with which each event is associated, and record the midpoint of this range as the time for the event. This procedure introduces an uncertainty of approximately \pm 30 s to the event time. Because we are searching for a signal with a very specific period of one sidereal day (or one half of a sidereal day), we maximize the sensitivity of our analysis by collecting the events into 12 bins of sidereal phase (each corresponding to 2 sidereal hours). The uncertainty of any individual event time is negligible compared to this bin width. Fig. 5.2 presents the sidereally binned event rates for the $e+>3$-jet and $\mu+>3$-jet signal samples. The origin of the time coordinate has been shifted to coincide with the vernal equinox of the year 2000 . 

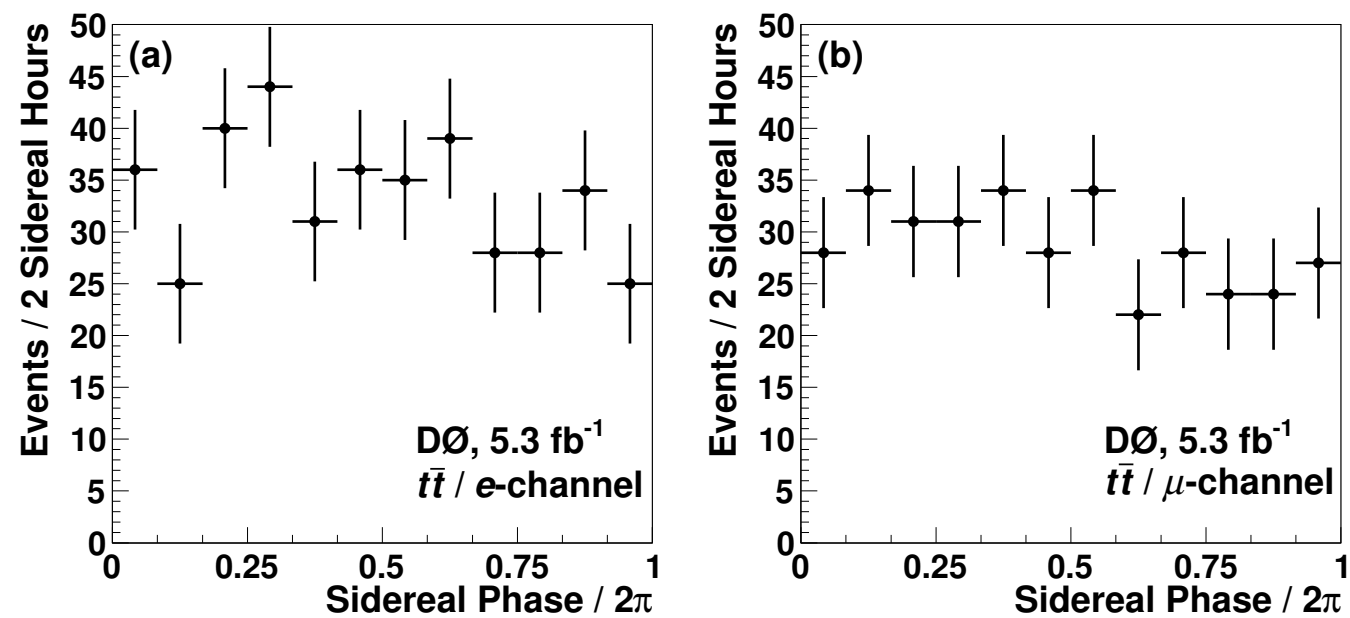

Figure 5.2: The number of $t \bar{t}$ candidates versus sidereal phase for (a) $e+>3$-jets events and (b) $\mu+>3$-jets events. The uncertainties reflect the sample size. 


\section{Chapter 6}

\section{Analysis at the DØ Experiment}

The cross section for semileptonic $t \bar{t}$ production and decay including Lorentz-violating contributions from the SME to the top quark sector, Eq. (3.25), can be approximated as the observed, time-averaged SM process cross section modulated by a time dependent function: $\sigma_{\mathrm{obs}}\left(1+f_{\mathrm{SME}}(t)\right)$. The contribution from the SME can be written as

$$
f_{\mathrm{SME}}(t)=\left(\left(c_{L}\right)_{\mu \nu}+\left(c_{R}\right)_{\mu \nu}\right) R_{\alpha}^{\mu}(t) R_{\beta}^{\nu}(t) A_{P}^{\alpha \beta}+\left(c_{L}\right)_{\mu \nu} R_{\alpha}^{\mu}(t) R_{\beta}^{\nu}(t) A_{F}^{\alpha \beta} .
$$

The SME coefficients $\left(c_{L}\right)_{\mu \nu}$ and $\left(c_{R}\right)_{\mu \nu}$ are defined in the Sun-centered reference frame. The matrices $A_{P}^{\alpha \beta}$ and $A_{F}^{\alpha \beta}$ represent averages over the event kinematics contributing to the SME components of the ratio $|\mathcal{M}|^{2} / P F \bar{F}$, detailed in Eqs. (3.22) and (3.23). It is most convenient to evaluate these terms in the reference frame of the $\mathrm{D} \varnothing$ detector and rotate them into the Sun-centered frame. This rotation, $R_{\alpha}^{\mu}(t)$, introduces the sidereal time dependence into the cross section. 


\subsection{Kinematic terms}

The summations in Eqs. (3.22) and (3.23) are carried out over those simulated $t \bar{t}$ events which pass fiducial acceptance, trigger selection, and analysis requirements on each reconstructed event. These criteria were discussed in the previous chapter, and are utilized to generate a collection of simulated events with which to calculate the elements of $A_{P}^{\alpha \beta}$ and $A_{F}^{\alpha \beta}$. Because of differences in various efficiencies between $e+$ jets events and $\mu+$ jets events, we calculate these matrices for each decay channel separately.

We generate two sets of Monte Carlo samples and simulate the response of the D $\varnothing$ detector to each event. We have 121675 events in the $e+$ jets sample and 138248 events in the $\mu+$ jets sample, providing ample statistics to minimize the uncertainty on these terms. We plot the contributions from each of the events to these sums and

collect them in Appendix B. Calculating the average for each term, we find $A_{P}^{\alpha \beta}$ and $A_{F}^{\alpha \beta}$ in the electron channel to be

$$
A_{P}^{\alpha \beta}=\left(\begin{array}{cccc}
0.218 \pm 0.001 & 0.000 \pm<0.001 & 0.000 \pm<0.001 & -0.003 \pm 0.002 \\
0.000 \pm<0.001 & 0.111 \pm<0.001 & 0.000 \pm<0.001 & 0.000 \pm 0.001 \\
0.000 \pm<0.001 & 0.000 \pm<0.001 & 0.111 \pm<0.001 & 0.000 \pm 0.001 \\
-0.003 \pm 0.002 & 0.000 \pm<0.001 & 0.000 \pm<0.001 & 1.184 \pm 0.001
\end{array}\right),
$$




$$
A_{F}^{\alpha \beta}=\left(\begin{array}{rrrr}
-8.075 \pm 0.092 & -0.010 \pm 0.019 & 0.031 \pm 0.023 & 0.042 \pm 0.064 \\
-0.010 \pm 0.019 & -0.323 \pm 0.013 & -0.003 \pm 0.012 & -0.023 \pm 0.013 \\
0.031 \pm 0.023 & -0.003 \pm 0.012 & -0.342 \pm 0.015 & -0.012 \pm 0.017 \\
0.042 \pm 0.064 & -0.023 \pm 0.013 & -0.012 \pm 0.017 & -2.126 \pm 0.048
\end{array}\right)
$$

and in the muon channel to be

$$
A_{P}^{\alpha \beta}=\left(\begin{array}{llll}
0.245 \pm 0.001 & 0.000 \pm<0.001 & 0.000 \pm<0.001 & 0.000 \pm 0.002 \\
0.000 \pm<0.001 & 0.113 \pm<0.001 & 0.000 \pm<0.001 & 0.000 \pm 0.001 \\
0.000 \pm<0.001 & 0.000 \pm<0.001 & 0.112 \pm<0.001 & 0.000 \pm 0.001 \\
0.000 \pm 0.002 & 0.000 \pm<0.001 & 0.000 \pm<0.001 & 1.200 \pm 0.001
\end{array}\right)
$$

$$
A_{F}^{\alpha \beta}=\left(\begin{array}{rrrr}
-8.570 \pm 0.068 & 0.042 \pm 0.022 & -0.021 \pm 0.022 & -0.011 \pm 0.037 \\
0.042 \pm 0.022 & -0.421 \pm 0.011 & -0.002 \pm 0.009 & -0.019 \pm 0.013 \\
-0.021 \pm 0.022 & -0.002 \pm 0.009 & -0.358 \pm 0.010 & -0.006 \pm 0.018 \\
-0.011 \pm 0.037 & -0.019 \pm 0.013 & -0.006 \pm 0.012 & -2.489 \pm 0.027
\end{array}\right)
$$

It is worth noting that these matrices are nearly diagonal and the $A^{x x}$ and $A^{y y}$ elements are effectively degenerate. The contributions to the off-diagonal elements are evenly distributed about zero, as can be seen in Figs. B.1-B.4 in Appendix B. The mathematical expressions for these terms, Eqs. (3.6)-(3.9), involve products of 
the form $p_{q_{1}}^{\mu} p_{q_{2}}^{\nu}$. These products tend to have an equal probability of being positive and negative in the off-diagonal cases, while the on-diagonal contributions of the form $\left(p^{\mu}\right)^{2}$ tend to be positive. The difference between the shape of the $A_{P}^{z z}$ term compared to the $t t, x x$, and $y y$ terms is likely due to a tendency for a small boost of the $t \bar{t}$ system along the beam direction in asymmetric initial state parton interactions. The degeneracy of $A^{x x}$ and $A^{y y}$ reflects the overall azimuthal symmetry of the $\mathrm{D} \varnothing$ detector.

While some of the distributions of the event-by-event contributions to these averages are broad, it is the average which contributes to $f_{\mathrm{SME}}(t)$. The quoted uncertainties on the elements of these matrices are purely statistical, and represent the uncertainty on the mean value of each term. For example, the standard deviation of the term $A_{P}^{\alpha \beta}$ is

$$
\begin{aligned}
\operatorname{sd}\left(A_{P}^{\alpha \beta}\right) & =\operatorname{sd}\left(\frac{1}{n_{\text {Acc }}} \sum_{k \in \text { Acc }}^{n_{\text {Acc }}}\left(\frac{\delta P_{p}^{\alpha \beta}}{P}+\frac{\delta P_{v}^{\alpha \beta}}{P}\right)\right) \\
& =\frac{1}{\sqrt{n_{\mathrm{Acc}}}} \operatorname{sd}\left(\frac{\delta P_{p}^{\alpha \beta}}{P}+\frac{\delta P_{v}^{\alpha \beta}}{P}\right) .
\end{aligned}
$$

\subsection{Reference frame transformation}

To determine the effects of the various SME coefficients, the matrices $A_{P}^{\alpha \beta}$ and $A_{F}^{\alpha \beta}$ must be transformed into the Sun-centered reference frame. The relative velocity of the two reference frames is negligible, so the transformation operation involves only rotations. We extend the rotation matrices defined in Eqs. (1.3)-(1.5) to four dimensions, yielding the following three transformations: 


$$
\begin{aligned}
& \tilde{R}_{x}(\phi)=\left(\begin{array}{cccc}
1 & 0 & 0 & 0 \\
0 & 1 & 0 & 0 \\
0 & 0 & \cos (\phi) & -\sin (\phi) \\
0 & 0 & \sin (\phi) & \cos (\phi)
\end{array}\right), \\
& \tilde{R}_{y}(\theta)=\left(\begin{array}{cccc}
1 & 0 & 0 & 0 \\
0 & \cos (\theta) & 0 & -\sin (\theta) \\
0 & 0 & 1 & 0 \\
0 & \sin (\theta) & 0 & \cos (\theta)
\end{array}\right), \\
& \tilde{R}_{z}(\psi)=\left(\begin{array}{cccc}
1 & 0 & 0 & 0 \\
0 & \cos (\psi) & \sin (\psi) & 0 \\
0 & -\sin (\psi) & \cos (\psi) & 0 \\
0 & 0 & 0 & 1
\end{array}\right) .
\end{aligned}
$$

The $\mathrm{D} \varnothing$ detector is located at the Fermilab accelerator facility, at a latitude of $40.175^{\circ} \mathrm{N}$. The $z$-axis is defined to point along the direction of travel of the proton beam, and the $y$-axis points vertically up. To relate this coordinate system to that of the Sun-centered reference frame, two parameters are required. The colatitude $(\chi)$ of the detector frame is the angle between the $y$-axis and the axis of Earth's rotation (the $Z$-axis); this is simply $90^{\circ}$ minus the latitude, or $49.825^{\circ}$. The orientation of the proton beam (the $z$-axis) is approximately $\alpha=42.192^{\circ}$ south of west.

The transformation from the $\mathrm{D} \varnothing$ frame to the Sun-centered frame is accomplished through four successive rotations. The coordinate system is first rotated about the detector $y$-axis by $\alpha$, followed by a rotation about the $x$-direction by $\pi / 2$. A second 
rotation about the $y$-axis by $\chi$ accounts for the latitude of the detector. A final rotation about the $Z$-axis by $\omega t$ (where $\omega$ is related to the sidereal period $T_{\text {side }}$ by $\left.\omega=2 \pi / T_{\text {side }}\right)$ introduces the sidereal time dependence. We have

$$
R(t)=\tilde{R}_{z}(\omega t) \tilde{R}_{y}(\chi) \tilde{R}_{x}(\pi / 2) \tilde{R}_{y}(\alpha) .
$$

Table 6.1 collects the constants of this rotation matrix: the colatitude $(\chi)$, orientation of the $\mathrm{D} \varnothing z$-axis $(\alpha)$, and the origin of the time coordinate $\left(t_{0}\right)$ chosen to coincide with the vernal equinox of the year 2000 .

Table 6.1: Parameters defining the orientation of the $\mathrm{D} \emptyset$ detector

\begin{tabular}{cc}
\hline \hline Parameter & Value \\
\hline Colatitude $(\chi)$ & $49.8255^{\circ}$ \\
D0 Rotation $(\alpha)$ & $42.192^{\circ}$ \\
$t_{0}$ & $953537400 \mathrm{~s}$ \\
\hline \hline
\end{tabular}

\subsection{Forms for $f_{\mathrm{SME}}(t)$}

The SME is a phenomenological formalism within which the terms parametrizing $\mathrm{CPT}$ and Lorentz violation are not constrained to couple with the same strength to all particle species. Many of these have already been tightly constrained, as discussed previously. We therefore impose the assumption that SME coefficients in all other particle sectors are zero. In order to maximize our sensitivity to SME coefficients in the top sector and set limits on their possible values, we estimate one component $(\mu, \nu)$ of each at a time. For example, to measure $\left(c_{L}\right)_{X X}$, we assume all other $\left(c_{L}\right)_{\mu \nu}$ are 
zero, as well as all $\left(c_{R}\right)_{\mu \nu}$. Except for the case of interference effects between different coefficients (which could lead to cancellation of observable effects on the total cross section), this procedure yields the maximum allowed values for each. Aside from such interference, if any of these assumptions were not fulfilled then the actual magnitude of each coefficient would be smaller than the limit we extract.

This procedure gives four basic model assumptions under which we perform our analysis: $\left(c_{R}\right)_{\mu \nu}=0,\left(c_{L}\right)_{\mu \nu}=0, c_{\mu \nu}=0$, and $d_{\mu \nu}=0$, where $c_{\mu \nu}$ and $d_{\mu \nu}$ are linear combinations of $\left(c_{R}\right)_{\mu \nu}$ and $\left(c_{L}\right)_{\mu \nu}$ defined in Eq. (3.1). Propagating these assumptions into Eq. (6.1), we have the possible forms for $f_{\mathrm{SME}}(t)$ listed in Table 6.2.

Table 6.2: $f_{\mathrm{SME}}(t)$ under different SME assumptions.

\begin{tabular}{cl}
\hline \hline Assumption & \multicolumn{1}{c}{$f_{\mathrm{SME}}(t)$} \\
\hline$\left(c_{R}\right)_{\mu \nu}=0$ & $\left(c_{L}\right)_{\mu \nu} R_{\alpha}^{\mu}(t) R_{\beta}^{\nu}(t)\left(A_{P}^{\alpha \beta}+A_{F}^{\alpha \beta}\right)$ \\
$\left(c_{L}\right)_{\mu \nu}=0$ & $\left(c_{R}\right)_{\mu \nu} R_{\alpha}^{\mu}(t) R_{\beta}^{\nu}(t)\left(A_{P}^{\alpha \beta}\right)$ \\
$d_{\mu \nu}=0$ & $c_{\mu \nu} R_{\alpha}^{\mu}(t) R_{\beta}^{\nu}(t)\left(A_{P}^{\alpha \beta}+\frac{1}{2} A_{F}^{\alpha \beta}\right)$ \\
$c_{\mu \nu}=0$ & $d_{\mu \nu} R_{\alpha}^{\mu}(t) R_{\beta}^{\nu}(t)\left(\frac{1}{2} A_{F}^{\alpha \beta}\right)$ \\
\hline \hline
\end{tabular}

The SME contribution to the cross section has the general form

$$
f_{\mathrm{SME}}(t)=C_{\mu \nu} R_{\alpha}^{\mu}(t) R_{\beta}^{\nu}(t) A^{\alpha \beta},
$$

where $C_{\mu \nu}$ represents the coefficient we wish to measure under each model assumption and $A^{\alpha \beta}$ represents the corresponding linear combination of $A_{P}^{\alpha \beta}$ and $A_{F}^{\alpha \beta}$. The matrices $\left(c_{L}\right)_{\mu \nu}$ and $\left(c_{R}\right)_{\mu \nu}$ are symmetric and traceless.

The expression for $f_{\mathrm{SME}}(t)$ can be simplified by noting that the matrices in Eqs. (6.2)-(6.5) are essentially diagonal, and the elements $A^{x x}$ and $A^{y y}$ nearly degenerate. A slight asymmetry in the acceptance of the muon spectrometer leads to 
the difference between these two terms in Eq. (6.5), but the effect on $f_{\mathrm{SME}}(t)$ from their difference is negligible. We take $A^{x x}$ to be the average of these elements, and Eq. (6.1) simplifies to

$$
f_{\mathrm{SME}}(t)=C_{\mu \nu}\left[R_{t}^{\mu}(t) R_{t}^{\nu}(t) A^{t t}+\left(R_{x}^{\mu}(t) R_{x}^{\nu}(t)+R_{y}^{\mu}(t) R_{y}^{\nu}(t)\right) A^{x x}+R_{z}^{\mu}(t) R_{z}^{\nu}(t) A^{z z}\right] .
$$

We perform the multiplications in Eq. (6.12) to determine the explicit form of $f_{\mathrm{SME}}(t)$ for each $\mu, \nu$ case.

For SME coefficients of the form $C_{T T}$, Eq. (6.12) yields

$$
f_{\mathrm{SME}}^{(T T)}(t)=C_{T T} A^{t t}=\text { constant }
$$

The TT components of $\left(c_{L}\right)_{\mu \nu}$ and $\left(c_{R}\right)_{\mu \nu}$ contribute only to the total cross section, and do not produce a time dependent signature. Similarly, for the $Z Z$ coefficients

$$
f_{\mathrm{SME}}^{(Z Z)}(t)=C_{Z Z}\left(\left(\sin ^{2}(\chi) \cos ^{2}(\alpha)+\cos ^{2}(\chi)\right) A^{x x}+\sin ^{2}(\chi) \sin ^{2}(\alpha) A^{z z}\right)=\mathrm{constant}
$$

only effects the average cross section. We do not attempt to measure these coefficients in this analysis.

The negligible contribution to the rotation matrix $R_{\alpha}^{\mu}(t)$ due to the velocity of the detector frame relative to the Sun-centered frame means the $R_{t}^{i}(t)$ components (where $i=X, Y, Z)$ and the $R_{i}^{T}(t)$ components $(i=x, y, z)$ are effectively zero. Together with the vanishing off-diagonal elements of $A_{P}^{\alpha \beta}$ and $A_{F}^{\alpha \beta}$, this gives 


$$
f_{\mathrm{SME}}^{(T i)}(t) \approx C_{T i} \times 0, i=X, Y, Z .
$$

We therefore do not expect this analysis to be sensitive to the $T X, T Y$, or $T Z$ elements of $\left(c_{L}\right)_{\mu \nu}$ and $\left(c_{R}\right)_{\mu \nu}$.

While we cannot measure either the $Z Z$ components or the coefficients involving the $T$ component in this analysis, the remaining components will couple to a time dependent oscillation in the $t \bar{t}$ cross section. For the coefficient $C_{X X}$, we find

$$
\begin{aligned}
f_{\mathrm{SME}}^{(X X)}(t) & =C_{X X}\left(b_{1} \cos ^{2}(\omega t)+b_{2} \sin ^{2}(\omega t)+2 b_{3} \sin (\omega t) \cos (\omega t)\right) \\
& =C_{X X}\left(\frac{b_{1}+b_{2}}{2}+\frac{b_{1}-b_{2}}{2} \cos (2 \omega t)+b_{3} \sin (2 \omega t)\right),
\end{aligned}
$$

and for $C_{Y Y}$

$$
\begin{aligned}
f_{\mathrm{SME}}^{(Y Y)}(t) & =C_{Y Y}\left(b_{1} \sin ^{2}(\omega t)+b_{2} \cos ^{2}(\omega t)-2 b_{3} \sin (\omega t) \cos (\omega t)\right) \\
& =C_{Y Y}\left(\frac{b_{1}+b_{2}}{2}-\frac{b_{1}-b_{2}}{2} \cos (2 \omega t)-b_{3} \sin (2 \omega t)\right) .
\end{aligned}
$$

The terms $b_{1}, b_{2}$, and $b_{3}$ depend on the colatitude and orientation of the detector, as well as the elements $A^{x x}$ and $A^{z z}$.

$$
\begin{aligned}
& b_{1}=\left(\cos ^{2}(\chi) \cos ^{2}(\alpha)+\sin ^{2}(\chi)\right) A^{x x}+\cos ^{2}(\chi) \sin ^{2}(\alpha) A^{z z}, \\
& b_{2}=\sin ^{2}(\alpha) A^{x x}+\cos ^{2}(\alpha) A^{z z}, \\
& b_{3}=\cos (\chi) \sin (\alpha) \cos (\alpha)\left(A^{z z}-A^{x x}\right) .
\end{aligned}
$$

Because the analysis is not sensitive to the $C_{T T}$ and $C_{Z Z}$ elements, those terms will 
be zero in each expression for $f_{\mathrm{SME}}(t)$ used to determine $C_{X X}$ and $C_{Y Y}$. To satisfy the requirement that the matrix of coefficients $C_{\mu \nu}$ be traceless, we choose $C_{Y Y}=-C_{X X}$. Combining Eqs. (6.16) and (6.17), the constant contributions cancel and we have

$$
f_{\mathrm{SME}}^{(X X)}(t)=2 C_{X X}\left(\frac{b_{1}-b_{2}}{2} \cos (2 \omega t)-b_{3} \sin (2 \omega t)\right) .
$$

The function $f_{\mathrm{SME}}(t)$ has a similar form for the coefficient $C_{X Y}$, with the symmetry condition $C_{Y X}=C_{X Y}$.

$$
f_{\mathrm{SME}}^{(X Y)}(t)=2 C_{X Y}\left(\frac{b_{1}-b_{2}}{2} \sin (2 \omega t)-b_{3} \cos (2 \omega t)\right) .
$$

Eq. (6.22) is the same as Eq. (6.21) under a shift in phase of the sidereal signal $\omega t \rightarrow \omega t+\pi / 4$, indicating that we can expect similar sensitivity to the two cases. These coefficients give rise to a signal that varies twice with the sidereal day.

The final SME coefficients to consider are $C_{X Z}$ and $C_{Y Z}$. With the appropriate symmetry requirements, the expressions for the modulation of the event rate are

$$
f_{\mathrm{SME}}^{(X Z)}(t)=2 C_{X Z}\left(b_{4} \cos (\omega t)+b_{5} \sin (\omega t)\right)
$$

and

$$
f_{\mathrm{SME}}^{(Y Z)}(t)=2 C_{Y Z}\left(b_{4} \sin (\omega t)-b_{5} \cos (\omega t)\right),
$$

respectively, with 


$$
\begin{aligned}
& b_{4}=\sin (\chi) \cos (\chi) \sin ^{2}(\alpha)\left(A^{x x}+A^{z z}\right), \\
& b_{5}=\sin (\chi) \sin (\alpha) \cos (\alpha)\left(A^{x x}-A^{z z}\right) .
\end{aligned}
$$

The expressions in Eqs. (6.23) and (6.24) vary once per sidereal day, and differ in sidereal phase by $\pi / 2$.

Equations (6.21)-(6.24) describe the expected modulation of the $t \bar{t}$ cross section due to the Lorentz violating contributions of the SME.

\subsection{Expected event rate}

The experiment directly measures the production rate of semileptonic $t \bar{t}$ events. The total number of $t \bar{t}$ events $\left(N_{t \bar{t}}\right)$ collected during a period of data collection $(\tau)$ is

$$
N_{t \bar{t}}=\int_{\tau} \mathcal{L}(t) \sigma(t) d t
$$

where $\mathcal{L}(t)$ is the instantaneous luminosity. The rate of $t \bar{t}$ events is therefore

$$
\frac{d N_{t \bar{t}}}{d t}=\mathcal{L}(t) \sigma(t)
$$

Because our analysis is only sensitive to SME coefficients of the form $C_{X X}, C_{X Y}$, $C_{X Z}$, and $C_{Y Z}$, the time dependent cross section has the general form

$$
\sigma(t)=\sigma_{\mathrm{ave}}\left(1+f_{\mathrm{SME}}(t)\right)
$$

where $\sigma_{\text {ave }}$ is the observed time-averaged cross section and $f_{\mathrm{SME}}(t)$ takes on one of the forms described in Eqs. (6.21)-(6.24) to give rise to a periodic modulation about 
this average. We define this average cross section in terms of the total number of $t \bar{t}$ events and the integrated luminosity, $\mathcal{L}_{\text {int }}$, and express the event rate as

$$
\frac{d N_{t \bar{t}}}{d t} \approx N_{t \bar{t}} \frac{\mathcal{L}(t)}{\mathcal{L}_{\mathrm{int}}}\left(1+f_{\mathrm{SME}}(t)\right)
$$

Eq. (6.30) describes the rate of $t \bar{t}$ events, but there is a non-negligible contamination in the observed rate due to irreducible background events. These events, principally from the $W+$ jets process, are kinematically similar to true $t \bar{t}$ events and cannot be distinguished from them. We therefore must consider their contribution to the total number of observed $t \bar{t}$ candidate events, $N_{\text {tot }}$. Because we assume any Lorentz violation due to SME effects belongs solely to the top quark sector, we expect the background (non-t $t \bar{t}$ ) event rate to depend only on fluctuations in the relative instantaneous luminosity.

$$
\begin{aligned}
\frac{d N_{B}}{d t} & =N_{B} \frac{\mathcal{L}(t)}{\mathcal{L}_{\mathrm{int}}} \\
& =\left(1-f_{S}\right) N_{\text {tot }} \frac{\mathcal{L}(t)}{\mathcal{L}_{\text {int }}}
\end{aligned}
$$

where the signal fraction $f_{S}$ is the relative number of true $t \bar{t}$ events estimated in the data set, such that $N_{t \bar{t}}=f_{S} N_{\text {tot }}$.

Combining the contributions from signal $(t \bar{t})$ and background (non- $t \bar{t}$ ) events, we find that

$$
\frac{d N_{\mathrm{tot}}}{d t} \approx N_{\mathrm{tot}} \frac{\mathcal{L}(t)}{\mathcal{L}_{\mathrm{int}}}\left(1+f_{S} f_{\mathrm{SME}}(t)\right)
$$

To maximize our sensitivity in a search for a signal varying periodically with 
sidereal time, we fold the data into twelve bins of sidereal phase (corresponding to two sidereal hours each). An event's sidereal phase is the fraction of the sidereal day corresponding to the time at which the event occurred, with $\phi(t)=\phi\left(t+n T_{\text {side }}\right)$. The number of events $N_{i}$ in a bin of sidereal phase is

$$
N_{i} \approx N_{\text {tot }} \frac{\mathcal{L}_{i}}{\mathcal{L}_{\text {int }}}\left(1+f_{S} f_{\mathrm{SME}}\left(\phi_{i}\right)\right)
$$

where $\mathcal{L}_{i}$ is the luminosity integrated over the appropriate bin whose center corresponds to sidereal phase $\phi_{i}$.

Fig. 6.1 shows the relative luminosity $\mathcal{L}_{i} / \mathcal{L}_{\text {int }}$ in each bin of sidereal phase. Slight differences in trigger requirements for the $e+$ jets and $\mu+$ jets selections give slight differences in this distribution for the two samples.
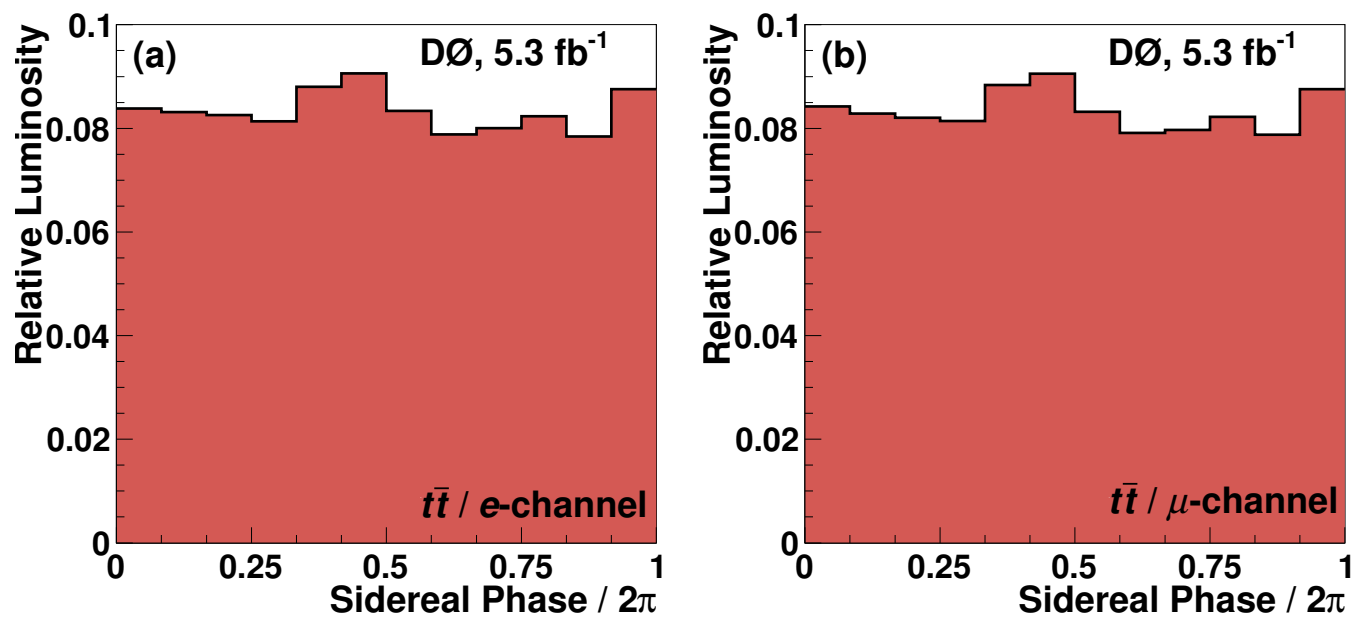

Figure 6.1: The relative luminosity $\mathcal{L}_{i} / \mathcal{L}_{\text {int }}$ by sidereal phase corresponding to (a) the $e+$ jets event selection and (b) the $\mu+$ jets event selection.

To directly compare the expectation for the sidereal time dependence $f_{\mathrm{SME}}(\phi)$ as described in Eqs. (6.21)-(6.24) to our data, we define the variable $R$ as 


$$
R_{i} \equiv \frac{1}{f_{S}}\left(\frac{N_{i} / N_{\text {tot }}}{\mathcal{L}_{i} / \mathcal{L}_{\text {int }}}-1\right)
$$

This distribution is the luminosity-corrected relative event rate versus sidereal phase. In the absence of any periodic time dependence, this distribution should be flat and distributed around $R=0$. A sidereal time dependence of the $t \bar{t}$ event rate would produce a sinusoidal variation in this distribution. Fig. 6.2 shows the resulting distributions for $R$ versus sidereal phase for the $e+$ jets and $\mu+$ jets signal samples. We use a $\chi$-square minimization method to compare our model $f_{\mathrm{SME}}\left(\phi_{i}\right)$ to each $R_{i}$ in order to extract the value of the SME coefficient under each assumption in Table 6.2.
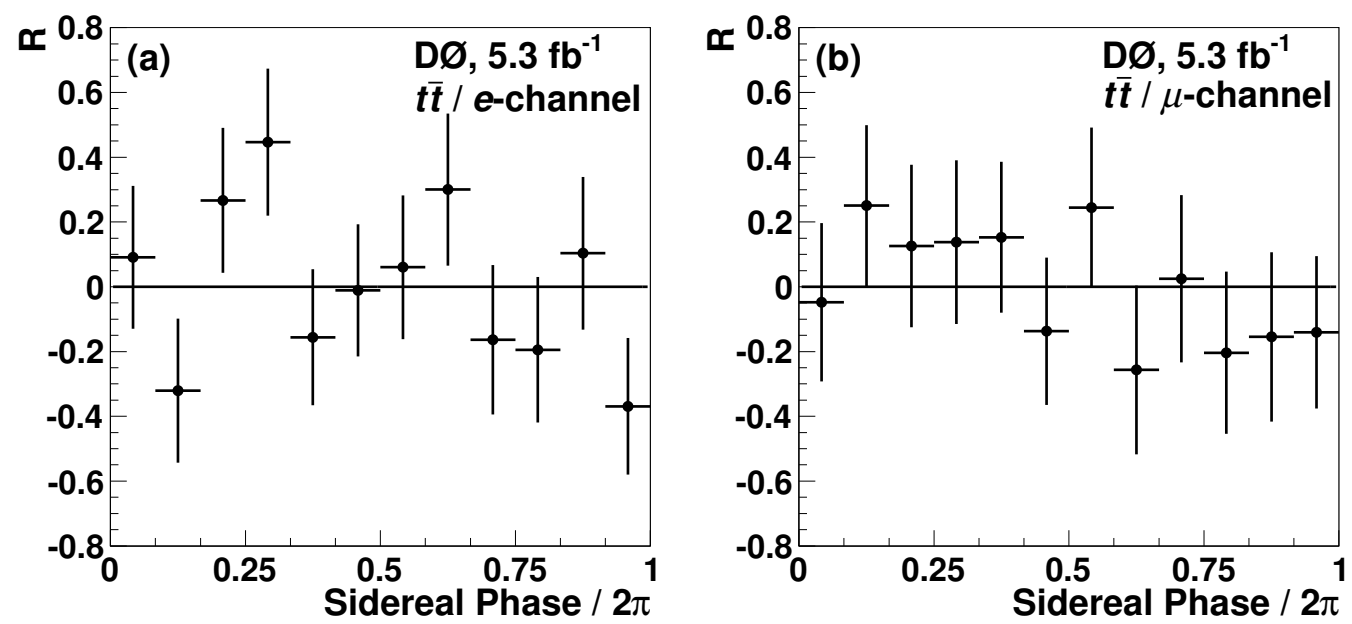

Figure 6.2: The dependence of $R$ on sidereal phase for (a) $e+>3$-jets $t \bar{t}$ candidates and (b) the $\mu+>3$-jets $t \bar{t}$ candidates. 


\subsection{Studies of Monte Carlo ensembles}

Before proceeding to measure the top sector SME coefficients, we ensure consistency of the analysis method by performing a series of fits using a large number of pseudoexperiments generated using standard Monte Carlo techniques. For each fit, we generate an ensemble of events comparable to that in our data set, impose a sidereal time dependence on the event rate, and extract the amplitude of sidereal oscillation in the corresponding $R$ distribution.

We use a rejection sampling technique to generate timestamps randomly distributed according to the expectation $1+f_{\mathrm{SME}}(t)$. This time dependence is modeled corresponding to the case where $\left(c_{L}\right)_{X X}=0.4,\left(c_{L}\right)_{\mu \nu}=0$ for all other values of $\mu$ and $\nu$, and all components of $\left(c_{R}\right)_{\mu \nu}=0$. We continue sampling until 401 event times have been selected, corresponding to the number of events in the $e+>3$-jets sample. The analysis is then performed to extract the value of $\left(c_{L}\right)_{X X}$ and this is compared to its input value. The entire process is repeated until a total of 10,000 experiments have been simulated.

Fig. 6.3a shows the distribution of value for $\left(c_{L}\right)_{X X}$ extracted from each ensemble, fit with a Gaussian function. The mean is near the input value of 0.4 , and the width of $\approx 0.17$ gives an indication of the expected statistical uncertainty for this search. The corresponding pull distribution (pull $=\left(\left(c_{L}\right)_{X X}-0.4\right) / \mathrm{sd}$, Fig. 6.3b) has an RMS near the expected value of unity. Each fit is performed using a $\chi$-square minimization method. An estimation of the quality of each fit is determined by calculating the probability of obtaining a $\chi^{2}$-value greater than that obtained from the fit. Fig. 6.3c

collects these probabilities for each ensemble. With 10 degrees of freedom in the fit (12 bins minus one degree for the fixed normalization and one for the extracted 
SME coefficient controlling the amplitude), the flat shape of this distribution reflects statistically consistent behavior of the fit method.

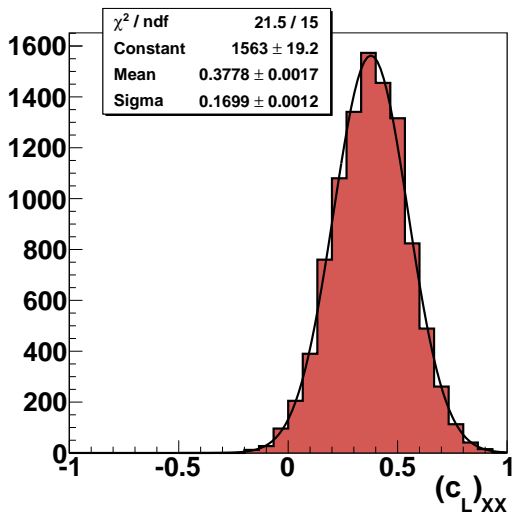

(a) Values for $\left(c_{L}\right)_{X X}$.

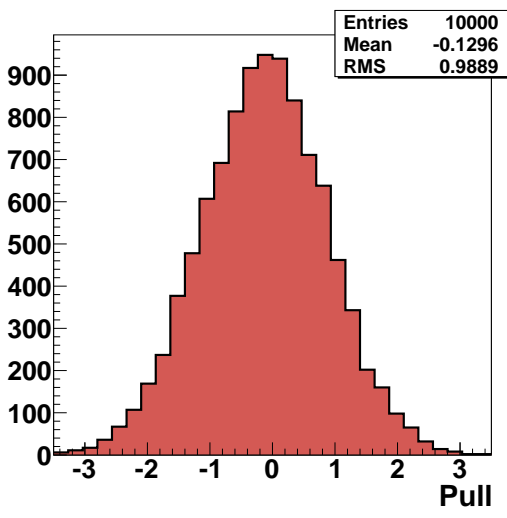

(b) Pull distribution.

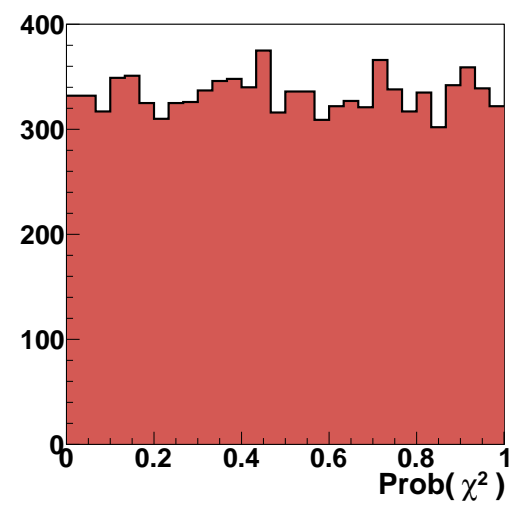

(c) Probability of the $\chi^{2}$ of the fit.

Figure 6.3: Parameters extracted from studies of 10,000 MC pseudo-experiments, each containing 401 events with an input value of $\left(c_{L}\right)_{X X}=0.4$ : (a) values of $\left(c_{L}\right)_{X X}$, (b) the pull $=\left(\left(c_{L}\right)_{X X}-0.4\right) / \mathrm{sd}$, and (c) the probability of the $\chi^{2}$ of the fit for each pseudo-experiment.

Both the statistically-significant difference in the mean of the extracted $\left(c_{L}\right)_{X X}$ in Fig. $6.3 \mathrm{a}$ and the non-zero mean in the pull distribution of Fig. $6.3 \mathrm{~b}$ indicate that the fit method appears to contain a small bias introduced by the finite sidereal bin size. 
We investigate this effect by considering the results described above for various other values of $\left(c_{L}\right)_{X X}$ and with sidereal phase divided into 12, 24, and 36 bins. Fig. 6.4 collects the results of this investigation. The fit behaves linearly (Fig. 6.4a) and the RMS of the pull distribution is consistently near unity (Fig. 6.4b) with respect to the input value for each choice of binning granularity.

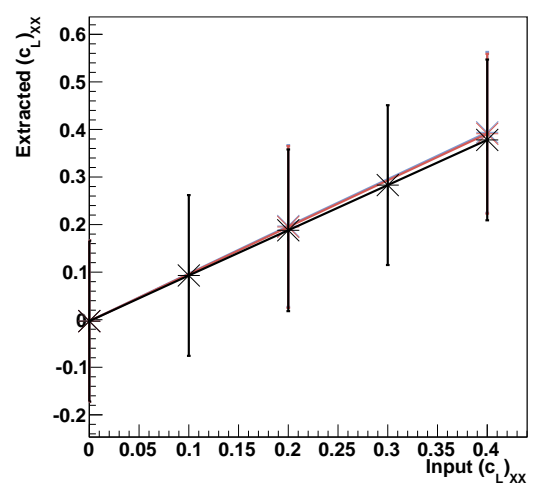

(a) Mean extracted value for $\left(c_{L}\right)_{X X}$.

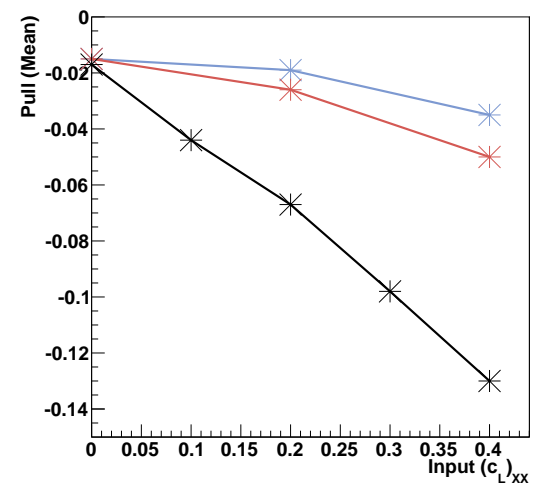

(b) Mean of the pull distribution.

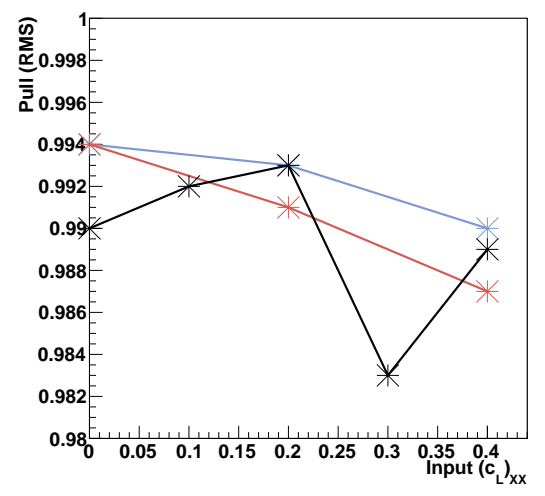

(c) RMS of the pull distribution.

Figure 6.4: Dependence of (a) the mean extracted value of $\left(c_{L}\right)_{X X}$, (b) the mean of the pull distribution, and (c) the RMS of the pull distribution on the input value of $\left(c_{L}\right)_{X X}$ for different values of sidereal binning: 12 bins (black), 24 bins (red), and 36 bins (blue). 
The mean of the pull distribution exhibits a dependence on the input value of the SME coefficient. The fact that this effect is enhanced by coarser sidereal binning indicates that this is indeed the source of the observed bias. The integrated luminosity is only available to this analysis accumulated into 12 bins of sidereal phase, so we calculate and apply a correction to the extracted SME parameter. This correction has the form

$$
C_{\mu \nu}=f_{\text {corr }} C_{\mu \nu}^{\mathrm{fit}},
$$

where we calculate $f_{\text {corr }} \approx 1.012$ for the case of a sinusoidal function varying with sidereal frequency (i.e. fits to extract coefficients of the form $C_{X Z}$ and $C_{Y Z}$ ), and $f_{\text {corr }} \approx 1.047$ for the case of a sinusoidal function which varies with twice the sidereal frequency (i.e. $C_{X X}$ and $C_{Y Y}$ ). Finally, the limited size of the collection of ensembles leads to a small shift of approximately -0.02 standard deviations in the pull distributions for an input value of 0.0 , but this shift approaches zero as the number of pseudo-experiments is increased.

We conclude that the analysis method is statistically consistent and behaves linearly with respect to the SME coefficients as expected. The correction described in Eq. (6.35) is sufficient to account for the small bias introduced by the choice to fold the data into 12 bins of sidereal phase, and we present the corrected values in all subsequent tables. 


\subsection{Amplitude of sidereal dependence}

The function $f_{\mathrm{SME}}(\phi)$ can be expressed in the form $A \cos (n \phi+\theta)$, where $n=2$ for the cases with SME coefficients of the form $C_{X X}$ and $C_{Y Y}$ while $n=1$ for the cases $C_{X Z}$ and $C_{Y Z}$. The amplitude and phase of this cosine function can be derived from Eqs. (6.21)-(6.24). The phase is fixed by the appropriate combination of the $b$-terms defined in Eqs. (6.18)-(6.20) and Eqs. (6.25)-(6.26). We fix the phase appropriate to each assumption in Table 6.2 and extract the amplitude of the resulting cosine function in order to determine the significance of any sidereal time dependence in the sidereally folded event rate. Tables 6.3-6.10 present these amplitudes under each of the sixteen model assumptions. We find that the amplitude of sidereal time dependence in the $\ell+>3$-jets data is consistent with zero within two standard deviations under all model assumptions in both lepton channels. We then fit Eqs. (6.21)-(6.24) to the $R$ distributions to directly extract values of SME coefficients. These are presented in Section 6.9 with both statistical and systematic uncertainties. 


\subsubsection{Electron amplitude results}

Table 6.3: Amplitude extracted from $e+>3$-jets $t \bar{t}$ candidates, with phase fixed appropriate to each coefficient case and assuming $\left(c_{R}\right)_{\mu \nu}=0$.

\begin{tabular}{ccc}
\hline \hline Coefficient Case & Amplitude \pm Uncertainty & Significance (sd) \\
\hline$X X$ & $-0.0653 \pm 0.0736$ & 0.887 \\
$X Y$ & $-0.0465 \pm 0.0741$ & 0.626 \\
$X Z$ & $0.1022 \pm 0.0707$ & 1.45 \\
$Y Z$ & $0.0153 \pm 0.0722$ & 0.213 \\
\hline \hline
\end{tabular}

Table 6.4: Amplitude extracted from $e+>3$-jets $t \bar{t}$ candidates, with phase fixed appropriate to each coefficient case and assuming $\left(c_{L}\right)_{\mu \nu}=0$.

\begin{tabular}{ccc}
\hline \hline Coefficient Case & Amplitude \pm Uncertainty & Significance (sd) \\
\hline$X X$ & $0.0653 \pm 0.0736$ & 0.887 \\
$X Y$ & $0.0465 \pm 0.0741$ & 0.626 \\
$X Z$ & $-0.1004 \pm 0.0710$ & 1.41 \\
$Y Z$ & $-0.0300 \pm 0.0718$ & 0.418 \\
\hline \hline
\end{tabular}

Table 6.5: Amplitude extracted from $e+>3$-jets $t \bar{t}$ candidates, with phase fixed appropriate to each coefficient case and assuming $d_{\mu \nu}=0$.

\begin{tabular}{ccc}
\hline \hline Coefficient Case & Amplitude \pm Uncertainty & Significance (sd) \\
\hline$X X$ & $0.0653 \pm 0.0736$ & 0.887 \\
$X Y$ & $0.0465 \pm 0.0741$ & 0.626 \\
$X Z$ & $-0.0837 \pm 0.0722$ & 1.16 \\
$Y Z$ & $-0.0654 \pm 0.0706$ & 0.925 \\
\hline \hline
\end{tabular}

Table 6.6: Amplitude extracted from $e+>3$-jets $t \bar{t}$ candidates, with phase fixed appropriate to each coefficient case and assuming $c_{\mu \nu}=0$.

\begin{tabular}{ccc}
\hline \hline Coefficient Case & Amplitude \pm Uncertainty & Significance (sd) \\
\hline$X X$ & $-0.0653 \pm 0.0736$ & 0.887 \\
$X Y$ & $-0.0465 \pm 0.0741$ & 0.626 \\
$X Z$ & $0.1012 \pm 0.0709$ & 1.43 \\
$Y Z$ & $0.0238 \pm 0.0720$ & 0.33 \\
\hline \hline
\end{tabular}




\subsubsection{Muon amplitude results}

Table 6.7: Amplitude extracted from $\mu+>3$-jets $t \bar{t}$ candidates, with phase fixed appropriate to each coefficient case and assuming $\left(c_{R}\right)_{\mu \nu}=0$.

\begin{tabular}{ccc}
\hline \hline Coefficient Case & Amplitude \pm Uncertainty & Significance (sd) \\
\hline$X X$ & $-0.0503 \pm 0.0794$ & 0.634 \\
$X Y$ & $0.0023 \pm 0.0799$ & 0.0288 \\
$X Z$ & $0.1008 \pm 0.0762$ & 1.32 \\
$Y Z$ & $-0.0572 \pm 0.0777$ & 0.735 \\
\hline \hline
\end{tabular}

Table 6.8: Amplitude extracted from $\mu+>3$-jets $t \bar{t}$ candidates, with phase fixed appropriate to each coefficient case and assuming $\left(c_{L}\right)_{\mu \nu}=0$.

\begin{tabular}{ccc}
\hline \hline Coefficient Case & Amplitude \pm Uncertainty & Significance (sd) \\
\hline$X X$ & $0.0503 \pm 0.0794$ & 0.634 \\
$X Y$ & $-0.0023 \pm 0.0799$ & 0.0288 \\
$X Z$ & $-0.1073 \pm 0.0766$ & 1.41 \\
$Y Z$ & $0.0436 \pm 0.0774$ & 0.563 \\
\hline \hline
\end{tabular}

Table 6.9: Amplitude extracted from $\mu+>3$-jets $t \bar{t}$ candidates, with phase fixed appropriate to each coefficient case and assuming $d_{\mu \nu}=0$.

\begin{tabular}{ccc}
\hline \hline Coefficient Case & Amplitude \pm Uncertainty & Significance (sd) \\
\hline$X X$ & $0.0503 \pm 0.0794$ & 0.634 \\
$X Y$ & $-0.0023 \pm 0.0799$ & 0.0288 \\
$X Z$ & $-0.0270 \pm 0.0769$ & 0.351 \\
$Y Z$ & $-0.1144 \pm 0.0770$ & 1.48 \\
\hline \hline
\end{tabular}

Table 6.10: Amplitude extracted from $\mu+>3$-jets $t \bar{t}$ candidates, with phase fixed appropriate to each coefficient case and assuming $c_{\mu \nu}=0$.

\begin{tabular}{ccc}
\hline \hline Coefficient Case & Amplitude \pm Uncertainty & Significance (sd) \\
\hline$X X$ & $-0.0503 \pm 0.0794$ & 0.634 \\
$X Y$ & $0.0023 \pm 0.0799$ & 0.0288 \\
$X Z$ & $0.1042 \pm 0.0764$ & 1.37 \\
$Y Z$ & $-0.0505 \pm 0.0776$ & 0.651 \\
\hline \hline
\end{tabular}




\subsection{Systematic uncertainties}

We expect a statistical uncertainty of around 0.17 on the magnitude of the SME coefficients for the 401 events in the $e+>3$-jet sample, and slightly larger for the 345event muon sample. Although this uncertainty will dominate our results, systematic factors can contribute as well.

The estimated fraction of events in the data which are true $t \bar{t}$ events is the dominant contribution to the systematic uncertainty on our extraction of the SME coefficients from the oscillation amplitudes. We determine the relevant signal fractions $\left(f_{S}\right)$ and their uncertainties from the results of the $t \bar{t}$ cross section analysis described in Reference [36], and treat these uncertainties as systematics in this study. The background from single top quark events could exhibit a sidereal time dependence due to SME effects. However, the phenomenological framework we employ does not describe single top production, and the relative contribution of single top events to the $t \bar{t}$ data is negligible $(\approx 1 \%)$. Table 6.11 lists the signal fractions for the two $\ell+>3$-jet data samples.

Table 6.11: Signal fractions $f_{S}$ for $\ell+>3$-jets $t \bar{t}$ candidates.

\begin{tabular}{ll}
\hline \hline Constant & Value \pm Uncertainty \\
\hline$f_{\mathrm{S}}(e+>3$-jets $)$ & $0.780 \pm 0.122$ \\
$f_{\mathrm{S}}(\mu+>3$-jets $)$ & $0.755 \pm 0.110$ \\
\hline \hline
\end{tabular}

While the statistical uncertainties on the diagonal elements of $A_{P}^{\alpha \beta}$ and $A_{F}^{\alpha \beta}$, Eqs. (6.2)-(6.5), are negligible compared to the statistical uncertainty due to the sizes of the data sets, these elements are subject to the same systematic effects as the event selection. We consider three leading sources of systematic uncertainty in our 
measurement of the kinematics of $t \bar{t}$ events: the jet energy scale (JES), jet energy resolution (JER), and jet identification (JETID). These factors have been identified as the primary sources affecting the distributions of momenta reconstructed in the detector [36]. We shift each of these factors up and down by one standard deviation in our Monte Carlo samples used to calculate $A_{P}^{\alpha \beta}$ and $A_{F}^{\alpha \beta}$ for each of the lepton channels and recalculate the elements. The effects of changing each of these sources on the diagonal elements of $A_{P}^{\alpha \beta}$ and $A_{F}^{\alpha \beta}$ are given in Appendix C.

As discussed previously, the $x x$ and $y y$ elements of $A_{P}^{\alpha \beta}$ and $A_{F}^{\alpha \beta}$ are nearly degenerate. This reflects the symmetry of the detector's $x$ - and $y$-axes. We therefore average the $x x$ and $y y$ elements calculated from the simulated event samples and take these as the values of $A_{P}^{x x}$ and $A_{F}^{x x}$ to be used in Eq. (6.12) to calculate the forms of $f_{\mathrm{SME}}(t)$. This procedure also minimizes the effects of a slight azimuthal asymmetry in the detector's muon acceptance, which is apparent in the $x x$ and $y y$ elements of $A_{F}^{\alpha \beta}$ calculated from the $\mu+$ jets simulation.

Table 6.12: A-matrix elements for $e+$ jets

\begin{tabular}{lc}
\hline \hline Element & Value $\pm($ Stat.+Sys. $)$ \\
\hline$\left(A_{P}\right)_{X X}$ & $0.1110 \pm 0.0011$ \\
$\left(A_{P}\right)_{Z Z}$ & $1.1841 \pm 0.0012$ \\
\hline$\left(A_{F}\right)_{X X}$ & $-0.3323 \pm 0.0200$ \\
$\left(A_{F}\right)_{Z Z}$ & $-2.1259 \pm 0.0509$ \\
\hline \hline
\end{tabular}

Tables 6.12 and 6.13 list the central values and combined uncertainties on the four elements of $A_{P}^{\alpha \beta}$ and $A_{F}^{\alpha \beta}$ which ultimately contribute to the forms of $f_{\mathrm{SME}}(t)$ used in this analysis. Although systematic effects like JES contribute to the distribution of components of reconstructed four-momenta in the detector, the elements of $A_{P}^{\alpha \beta}$ 
Table 6.13: $A$-matrix elements for $\mu+$ jets

\begin{tabular}{lc}
\hline \hline Element & Value \pm (Stat.+Sys.) \\
\hline$\left(A_{P}\right)_{X X}$ & $0.1122 \pm 0.0009$ \\
$\left(A_{P}\right)_{Z Z}$ & $1.1995 \pm 0.0014$ \\
\hline$\left(A_{F}\right)_{X X}$ & $-0.3896 \pm 0.0173$ \\
$\left(A_{F}\right)_{Z Z}$ & $-2.4885 \pm 0.0300$ \\
\hline
\end{tabular}

and $A_{F}^{\alpha \beta}$ reflect only averages of these components over the detector acceptance. Such averages are not very sensitive to changes in these kinematic parameters at magnitudes appropriate to $\mathrm{D} \emptyset$ detector performance. Thus, the relative uncertainty on elements of $A_{P}^{\alpha \beta}$ and $A_{F}^{\alpha \beta}$ is comparable to the statistical uncertainty on those elements, and can be neglected when compared to the systematic uncertainty on the signal fractions $f_{S}$.

Finally, the coordinates of the $\mathrm{D} \emptyset$ detector necessary to calculate $f_{\mathrm{SME}}(t)$ (collected in Table 6.1) carry negligible uncertainties.

\subsection{Cross-checks}

The efficiency to trigger and reconstruct a $t \bar{t}$ candidate event could potentially change with time. Our analysis must confirm that such effects do not contribute to the sidereally-binned $t \bar{t}$ event rate. Effects that cause time dependent efficiencies could take the form of either random changes or periodic modulation. Over a long period of time, any random changes in efficiency will contribute with equal likelihood to each bin of sidereal phase and will therefore not contribute to any periodic modulation of this distribution. Alternatively, various environmental factors like temperature variations between day and night could potentially introduce a periodic effect in 
the event selection efficiency. We test for such time dependence contributing to the sidereally-folded event rate by examining $t \bar{t}$ candidates in exclusive event selection of different jet multiplicities.

\subsubsection{Background-enhanced samples}

We test for sidereal time dependence in the $R$ distributions of the lepton $+n$-jets channels, where $n=2$ or 3 and the lepton is either an electron or a muon. We generate $R$ distributions for each of these four cross-check channels with the same approach used for the signal channels. The $t \bar{t}$ signal fractions for each of these selections are collected in Table 6.14.

Table 6.14: $t \bar{t}$ fractions for the $\ell+n$-jets samples, $n=2,3$.

\begin{tabular}{lc}
\hline \hline Constant & Value \\
\hline$f_{\mathrm{S}}(e+2$-jets $)$ & $0.130 \pm 0.017$ \\
$f_{\mathrm{S}}(\mu+2$-jets $)$ & $0.111 \pm 0.015$ \\
$f_{\mathrm{S}}(e+3$-jets $)$ & $0.454 \pm 0.056$ \\
$f_{\mathrm{S}}(\mu+3$-jets $)$ & $0.445 \pm 0.057$ \\
\hline \hline
\end{tabular}

Events in these cross-check channels consist mostly of non- $t \bar{t}$ events, principally from irreducible $W+$ jets events in which we expect no sidereal effects from LV. Such events are topologically similar to the signal $t \bar{t}$ events, exhibiting comparable kinematic distributions, and are subject to the same trigger, acceptance, and selection efficiencies as true $t \bar{t}$ events. We have imposed the assumption that all SME coefficients outside the top sector are zero; nonetheless any hypothetical Lorentz-violating contributions from $W+$ jets events would have to exactly cancel any sidereal dependence from detector effects to invalidate our conclusions. As this situation is quite 
unlikely, these low-signal $\ell+2$-jets and $\ell+3$-jets event selections therefore provide a handle on detector efficiencies that could effect our measurement in the signal $\ell+>3$ jets channels.

We extract the amplitudes for any time dependent oscillations in the $R$ distributions, corresponding to the same parametrizations used in Tables 6.3-6.10, and the results of these fits are presented in Appendix D. We test the hypothesis that the fitted amplitudes arise from statistical fluctuations by calculating the $\chi^{2}$ probability for consistency of the 4 independently measured amplitudes with the assumption of no sidereal dependence (Table D.17). For each model assumption, the ensemble of fits is consistent with no time dependence in the $R$ distribution at levels of probability in the range $6 \%-38 \%$.

While the four cross-check channels taken together provide satisfactory evidence that there are no contributions to the sidereal event rate from detector effects, the $e+2$-jets data alone exhibit some deviation from no sidereal time dependence in some of the fits. For example, a fit of a cosine function with phase appropriate to the model assumption $d_{\mu \nu}=0$ for the case $c_{Y Z}=-c_{Z Y}$ yields an amplitude that is 2.8 sd from zero. This measurement is close to the 3 sd threshold generally accepted as an indication of an effect not attributable to a statistical fluctuation.

To thoroughly investigate the source of the apparent effect in the $e+2$-jets sample, we divide our data into periods corresponding to Run IIa (the first $\approx 1 \mathrm{fb}^{-1}$ of data) and Run IIb (the latter $\approx 4.3 \mathrm{fb}^{-1}$ of data). Significant improvements to the DØ detector were made between these periods, making it the leading order transition point for any possible changes in data quality which could have introduced or resolved uncertainties in detector efficiencies. 
We present the results of this Run IIa/Run IIb separation in Appendix E and find that the largest deviations from a null result of the fitted amplitudes occur in the Run IIa $e+n$-jets samples. We again test the hypothesis that the fitted deviations arise from statistical fluctuations, calculating the consistency with no sidereal dependence of all 12 independently measured amplitudes $(\ell+2$-jets, $\ell+3$-jets, and $\ell+>3$-jets, for $\ell=e, \mu$ in Run IIa and Run IIb). As shown in Table E.49, these $\chi^{2}$ probabilities lie in the range $0.7 \%-35 \%$. The hypothesis of a statisical origin for the observed deviations is not badly disfavored. In the absence of any compelling evidence of problems with the Run IIa $e+$ jets sample, we therefore conclude that the results of these cross-checks give no conclusive indication of the presence of a time dependent efficiency that would affect our measurements of the SME coefficients governing LV in the top sector.

\subsubsection{Binning effects}

Any residual non-sidereal time dependence is greatly suppressed by folding the data set into twelve bins of sidereal phase. The magnitude of any residual contribution to this folded distribution depends inversely on the difference between the period of such a time dependent efficiency and the sidereal period. Most problematic would be an unexpected time dependent effect with a period close to that of a sidereal day. The worst realistic case would be a modulation of the detector efficiency that varies with a 24 hour period, such as a day-to-night temperature variation or similar effect. However, because the data set spans approximately seven years, any contributions from such an effect would be suppressed by a factor of about 10 . To affect our measurements, the $\mathrm{D} \emptyset$ detector would have to have experienced a highly unlikely periodic variation of the efficiency by approximately $75 \%$ over 24 hours. No periodic 
effects of this magnitude have ever been observed in the detection efficiencies for reconstructed objects considered in this analysis.

\subsection{Measured SME coefficients}

Confident that there are no time dependent efficiencies that will affect our results, we proceed to extract the values of the SME coefficients parametrizing LV in the top sector from the $e+>3$-jet and $\mu+>3$-jet $t \bar{t}$ event selections. We present these coefficients under each model assumption, along with their statistical and systematic uncertainties and the statistical quality of the fit, in Tables $6.15-6.22$. The corresponding plots for the electron and muon samples are collected in Appendices F.1 and F.2, respectively.

The SME coefficients parametrizing LV in the top sector are independent of the flavor of the lepton from the $W$ boson in the top quark or antitop quark decay. To maximize our sensitivity, we perform a simultaneous fit to both the $e+>3$-jets and $\mu+>3$-jets data sets to extract our final measurements of these SME coefficients. Tables 6.23-6.26 collect the results of these simultaneous fits, with corresponding plots presented in Appendix F.3.

The only non-negligible systematic uncertainty arises from the signal fraction $f_{S}$ for each sample. This parameter is varied by $\pm 1 \mathrm{sd}$, and the resulting variations in the central values of the fit results determine the systematic uncertainty on the SME coefficients. 


\subsubsection{Electron sample results}

Table 6.15: $\left(c_{L}\right)_{\mu \nu}$ extracted from $e+$ jets $t \bar{t}$ candidates, assuming $\left(c_{R}\right)_{\mu \nu}=0$.

\begin{tabular}{lcc}
\hline \hline Coefficient & Value \pm Stat. \pm Sys. & $\chi^{2} / \mathrm{nDoF}$ \\
\hline$\left(c_{L}\right)_{X X}$ & $-0.158 \pm 0.178 \pm 0.025$ & $13.5 / 10$ \\
$\left(c_{L}\right)_{X Y}$ & $-0.112 \pm 0.179 \pm 0.018$ & $13.9 / 10$ \\
$\left(c_{L}\right)_{X Z}$ & $0.174 \pm 0.120 \pm 0.028$ & $12.2 / 10$ \\
$\left(c_{L}\right)_{Y Z}$ & $0.026 \pm 0.122 \pm 0.004$ & $14.3 / 10$ \\
\hline \hline
\end{tabular}

Table 6.16: $\left(c_{R}\right)_{\mu \nu}$ extracted from $e+$ jets $t \bar{t}$ candidates, assuming $\left(c_{L}\right)_{\mu \nu}=0$.

\begin{tabular}{lcc}
\hline \hline Coefficient & Value \pm Stat. \pm Sys. & $\chi^{2} / \mathrm{nDoF}$ \\
\hline$\left(c_{R}\right)_{X X}$ & $0.106 \pm 0.119 \pm 0.017$ & $13.5 / 10$ \\
$\left(c_{R}\right)_{X Y}$ & $0.075 \pm 0.120 \pm 0.012$ & $13.9 / 10$ \\
$\left(c_{R}\right)_{X Z}$ & $-0.129 \pm 0.091 \pm 0.021$ & $12.3 / 10$ \\
$\left(c_{R}\right)_{Y Z}$ & $-0.038 \pm 0.092 \pm 0.006$ & $14.1 / 10$ \\
\hline \hline
\end{tabular}

Table 6.17: $c_{\mu \nu}$ extracted from $e+$ jets $t \bar{t}$ candidates, assuming $d_{\mu \nu}=0$.

\begin{tabular}{lcc}
\hline \hline Coefficient & Value \pm Stat. \pm Sys. & $\chi^{2} / \mathrm{nDoF}$ \\
\hline$c_{X X}$ & $0.645 \pm 0.727 \pm 0.103$ & $13.5 / 10$ \\
$c_{X Y}$ & $0.459 \pm 0.732 \pm 0.073$ & $13.9 / 10$ \\
$c_{X Z}$ & $-0.781 \pm 0.674 \pm 0.125$ & $13 / 10$ \\
$c_{Y Z}$ & $-0.610 \pm 0.660 \pm 0.098$ & $13.4 / 10$ \\
\hline \hline
\end{tabular}

Table 6.18: $d_{\mu \nu}$ extracted from $e+$ jets $t \bar{t}$ candidates, assuming $c_{\mu \nu}=0$.

\begin{tabular}{lcc}
\hline \hline Coefficient & Value \pm Stat. \pm Sys. & $\chi^{2} / \mathrm{nDoF}$ \\
\hline$d_{X X}$ & $-0.127 \pm 0.142 \pm 0.020$ & $13.5 / 10$ \\
$d_{X Y}$ & $-0.090 \pm 0.143 \pm 0.014$ & $13.9 / 10$ \\
$d_{X Z}$ & $0.149 \pm 0.104 \pm 0.024$ & $12.3 / 10$ \\
$d_{Y Z}$ & $0.035 \pm 0.105 \pm 0.006$ & $14.2 / 10$ \\
\hline \hline
\end{tabular}




\subsubsection{Muon sample results}

Table 6.19: $\left(c_{L}\right)_{\mu \nu}$ extracted from $\mu+$ jets $t \bar{t}$ candidates, assuming $\left(c_{R}\right)_{\mu \nu}=0$.

\begin{tabular}{lrl}
\hline \hline Coefficient & \multicolumn{1}{c}{ Value \pm Stat. \pm Sys. } & $\chi^{2} / \mathrm{nDoF}$ \\
\hline$\left(c_{L}\right)_{X X}$ & $-0.089 \pm 0.141 \pm 0.013$ & $5.32 / 10$ \\
$\left(c_{L}\right)_{X Y}$ & $0.004 \pm 0.142 \pm 0.001$ & $5.72 / 10$ \\
$\left(c_{L}\right)_{X Z}$ & $0.129 \pm 0.097 \pm 0.019$ & $3.98 / 10$ \\
$\left(c_{L}\right)_{Y Z}$ & $-0.073 \pm 0.099 \pm 0.011$ & $5.18 / 10$ \\
\hline \hline
\end{tabular}

Table 6.20: $\left(c_{R}\right)_{\mu \nu}$ extracted from $\mu+$ jets $t \bar{t}$ candidates, assuming $\left(c_{L}\right)_{\mu \nu}=0$.

\begin{tabular}{lcl}
\hline \hline Coefficient & \multicolumn{1}{c}{ Value \pm Stat. \pm Sys. } & \multicolumn{1}{c}{$\chi^{2} / \mathrm{nDoF}$} \\
\hline$\left(c_{R}\right)_{X X}$ & $0.083 \pm 0.131 \pm 0.012$ & $5.32 / 10$ \\
$\left(c_{R}\right)_{X Y}$ & $-0.004 \pm 0.132 \pm 0.001$ & $5.72 / 10$ \\
$\left(c_{R}\right)_{X Z}$ & $-0.141 \pm 0.100 \pm 0.021$ & $3.75 / 10$ \\
$\left(c_{R}\right)_{Y Z}$ & $0.057 \pm 0.101 \pm 0.008$ & $5.41 / 10$ \\
\hline \hline
\end{tabular}

Table 6.21: $c_{\mu \nu}$ extracted from $\mu+$ jets $t \bar{t}$ candidates, assuming $d_{\mu \nu}=0$.

\begin{tabular}{lcc}
\hline \hline Coefficient & Value \pm Stat. \pm Sys. & $\chi^{2} / \mathrm{nDoF}$ \\
\hline$c_{X X}$ & $2.387 \pm 3.769 \pm 0.356$ & $5.32 / 10$ \\
$c_{X Y}$ & $-0.110 \pm 3.801 \pm 0.016$ & $5.72 / 10$ \\
$c_{X Z}$ & $-0.563 \pm 1.599 \pm 0.084$ & $5.6 / 10$ \\
$c_{Y Z}$ & $-2.378 \pm 1.609 \pm 0.354$ & $3.53 / 10$ \\
\hline \hline
\end{tabular}

Table 6.22: $d_{\mu \nu}$ extracted from $\mu+$ jets $t \bar{t}$ candidates, assuming $c_{\mu \nu}=0$.

\begin{tabular}{lcc}
\hline \hline Coefficient & Value \pm Stat. \pm Sys. & $\chi^{2} / \mathrm{nDoF}$ \\
\hline$d_{X X}$ & $-0.086 \pm 0.136 \pm 0.013$ & $5.32 / 10$ \\
$d_{X Y}$ & $0.004 \pm 0.137 \pm 0.001$ & $5.72 / 10$ \\
$d_{X Z}$ & $0.136 \pm 0.099 \pm 0.020$ & $3.86 / 10$ \\
$d_{Y Z}$ & $-0.065 \pm 0.100 \pm 0.010$ & $5.3 / 10$ \\
\hline \hline
\end{tabular}




\subsubsection{Simultaneous fit results}

Table 6.23: $\left(c_{L}\right)_{\mu \nu}$ extracted from simultaneous fits to $e+$ jets and $\mu+$ jets $t \bar{t}$ candidates, assuming $\left(c_{R}\right)_{\mu \nu}=0$.

\begin{tabular}{lcc}
\hline \hline Coefficient & Value \pm Stat. \pm Sys. & $\chi^{2} / \mathrm{nDoF}$ \\
\hline$\left(c_{L}\right)_{X X}$ & $-0.116 \pm 0.111 \pm 0.018$ & $18.9 / 21$ \\
$\left(c_{L}\right)_{X Y}$ & $-0.041 \pm 0.111 \pm 0.006$ & $19.9 / 21$ \\
$\left(c_{L}\right)_{X Z}$ & $0.147 \pm 0.076 \pm 0.022$ & $16.3 / 21$ \\
$\left(c_{L}\right)_{Y Z}$ & $-0.034 \pm 0.077 \pm 0.005$ & $19.8 / 21$ \\
\hline \hline
\end{tabular}

Table 6.24: $\left(c_{R}\right)_{\mu \nu}$ extracted from simultaneous fits to $e+$ jets and $\mu+$ jets $t \bar{t}$ candidates, assuming $\left(c_{L}\right)_{\mu \nu}=0$.

\begin{tabular}{lcc}
\hline \hline Coefficient & Value \pm Stat. \pm Sys. & $\chi^{2} / \mathrm{nDoF}$ \\
\hline$\left(c_{R}\right)_{X X}$ & $0.096 \pm 0.088 \pm 0.015$ & $18.9 / 21$ \\
$\left(c_{R}\right)_{X Y}$ & $0.039 \pm 0.089 \pm 0.006$ & $19.8 / 21$ \\
$\left(c_{R}\right)_{X Z}$ & $-0.135 \pm 0.068 \pm 0.021$ & $16.1 / 21$ \\
$\left(c_{R}\right)_{Y Z}$ & $0.005 \pm 0.068 \pm 0.001$ & $20 / 21$ \\
\hline \hline
\end{tabular}

Table 6.25: $c_{\mu \nu}$ extracted from simultaneous fits to $e+$ jets and $\mu+$ jets $t \bar{t}$ candidates, assuming $d_{\mu \nu}=0$.

\begin{tabular}{lcc}
\hline \hline Coefficient & Value \pm Stat. \pm Sys. & $\chi^{2} / \mathrm{nDoF}$ \\
\hline$c_{X X}$ & $0.707 \pm 0.713 \pm 0.114$ & $19 / 21$ \\
$c_{X Y}$ & $0.438 \pm 0.719 \pm 0.070$ & $19.7 / 21$ \\
$c_{X Z}$ & $-0.749 \pm 0.621 \pm 0.118$ & $18.6 / 21$ \\
$c_{Y Z}$ & $-0.865 \pm 0.610 \pm 0.140$ & $18 / 21$ \\
\hline \hline
\end{tabular}

Table 6.26: $d_{\mu \nu}$ extracted from simultaneous fits to $e+$ jets and $\mu+$ jets $t \bar{t}$ candidates, assuming $c_{\mu \nu}=0$.

\begin{tabular}{lcc}
\hline \hline Coefficient & Value \pm Stat. \pm Sys. & $\chi^{2} / \mathrm{nDoF}$ \\
\hline$d_{X X}$ & $-0.106 \pm 0.099 \pm 0.016$ & $18.9 / 21$ \\
$d_{X Y}$ & $-0.041 \pm 0.099 \pm 0.006$ & $19.9 / 21$ \\
$d_{X Z}$ & $0.142 \pm 0.072 \pm 0.022$ & $16.1 / 21$ \\
$d_{Y Z}$ & $-0.018 \pm 0.073 \pm 0.003$ & $20 / 21$ \\
\hline
\end{tabular}




\section{Chapter 7}

\section{Prospective Analysis at the ATLAS Experiment}

As one of the general purpose particle detectors located at the Large Hadron Collider (LHC), the ATLAS detector has already surpassed the DØ experiment in the volume of $t \bar{t}$ events collected and analyzed. The LHC delivered proton-proton collisions with energies $\sqrt{s}=7 \mathrm{TeV}$ during 2011, followed by an increase to $\sqrt{s}=8 \mathrm{TeV}$ beginning in 2012. Consequently, the production cross section for $t \bar{t}$ pairs is significantly higher at ATLAS than at D $\varnothing$. (For example, $\sigma_{t \bar{t}}=177_{-10}^{+11} \mathrm{pb}$ in $p p$ collisions at $\sqrt{s}=7 \mathrm{TeV}[41]$ versus $\sigma_{t \bar{t}}=7.78_{-0.64}^{+0.77} \mathrm{pb}$ in $p \bar{p}$ collisions at $\sqrt{s}=1.98 \mathrm{TeV}$ [36]). A measurement of Lorentz violation in the top sector using the ATLAS detector will be less constrained by statistical uncertainties and provide better limits on the relevant SME coefficients than those determined with the $\mathrm{D} \varnothing$ experiment.

Because the contribution to this analysis at ATLAS from statistical uncertainty is smaller than that experienced with the D $\varnothing$ study, systematic uncertainties may play a 
more prominent role. There are also several differences between conditions at ATLAS versus $D \varnothing$ that must be considered. The distributions of parton momenta associated with the final state particles from a $t \bar{t}$ event reconstructed in the ATLAS detector will differ due to the higher center of mass energy of the collisions, potentially affecting

elements of the $A_{P}^{\alpha \beta}$ and $A_{F}^{\alpha \beta}$ matrices. This would provide a different contribution to the amplitude of any LV-induced sidereal time dependence and affect the sensitivity to SME coefficients. Furthermore, as of 2012 the ATLAS data taking period only spans a range of about two years, potentially giving a smaller suppression of any 24-hour periodic effects on the sidereally folded event rate.

We present a discussion of this analysis as it might be performed using data collected by the ATLAS experiment. We consider the qualitative and quantitative differences between $t \bar{t}$ events at the two experiments, and develop an expectation for the limits on the top-sector SME parameters $\left(c_{L}\right)_{\mu \nu}$ and $\left(c_{R}\right)_{\mu \nu}$ that could be determined from ATLAS data. We also propose an alternate analysis that could further improve on these expectations by utilizing more detailed luminosity data than was available to the $\mathrm{D} \varnothing$ analysis.

\subsection{The ATLAS detector}

The ATLAS detector [42] ( $\underline{\mathrm{A}} \underline{\text { Toroidal }} \underline{\mathrm{LHC}} \underline{\mathrm{A}} \mathrm{pparatu} \underline{\mathrm{S}}$ ) is a particle detector designed to study the products of proton-proton collisions delivered by the Large Hadron Collider (LHC). It is the result of the collaborative effort of roughly 3,000 scientists and engineers from more than 170 institutions across almost 40 countries. Similar to D $\varnothing$, ATLAS consists of several layers of sophisticated tracking and calorimetric sensors 
arrayed in a cylindrical geometry around the $p p$ interaction point. Figure 7.1 shows a cut-away view of the ATLAS detector, with the major subsystems labeled.

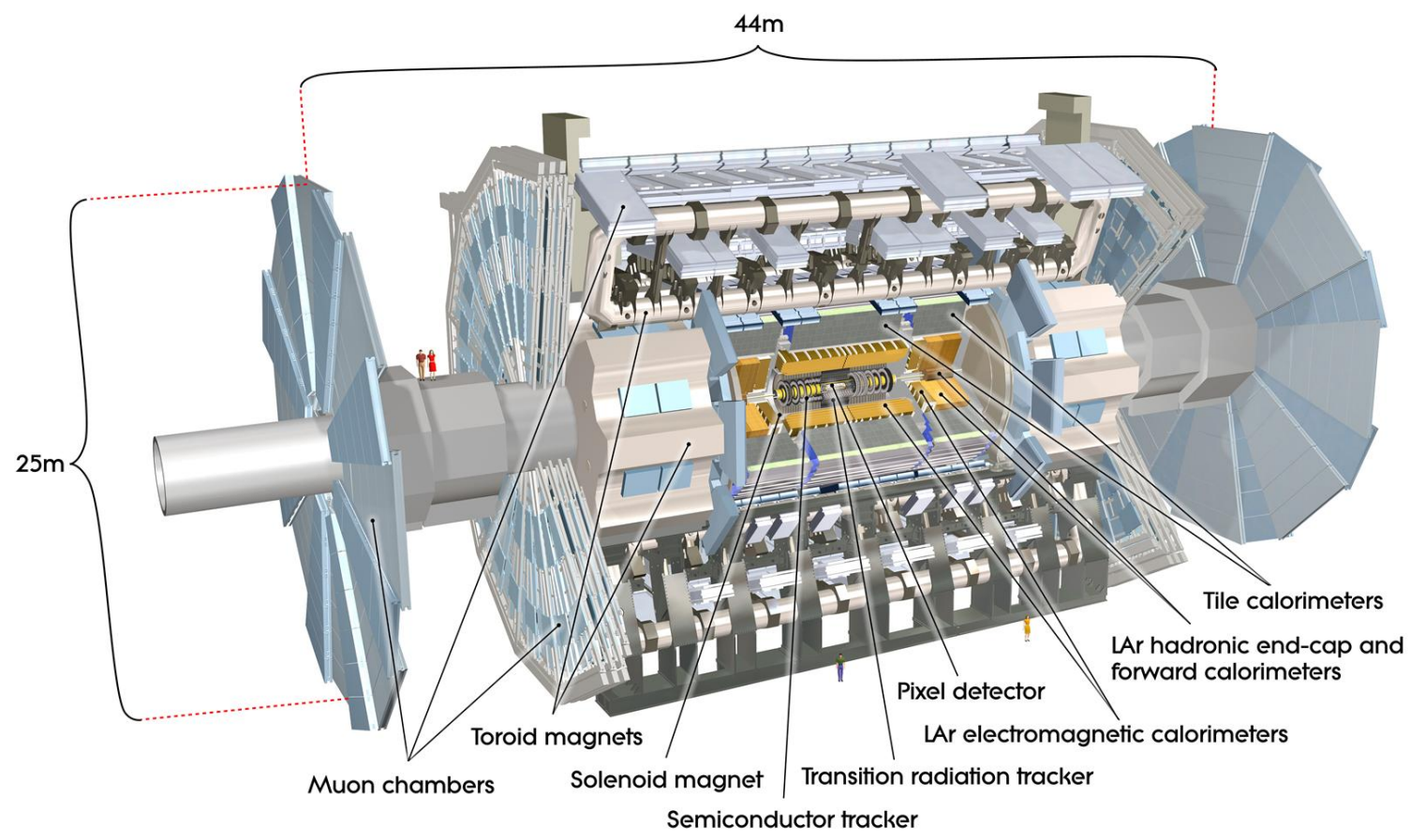

Figure 7.1: The ATLAS detector and its major subsystems. Several technicians next to the detector give a sense of the immense scale of the apparatus. (ATLAS Experiment (c) 2012 CERN)

\subsubsection{Large Hadron Collider}

The collisions which the ATLAS experiment investigates begin with the acceleration of protons through a chain of synchrotrons at the CERN laboratory. Figure 7.2 shows the various accelerator stages at CERN. Protons collided in the LHC begin at LINAC 2 (LINAC 3 provides $\mathrm{Pb}$ ions for heavy ion collisions at the LHC), are accelerated by the Proton Synchrotron Booster (PSB, or "Booster") to $1.4 \mathrm{GeV}$, and passed to the 
Proton Synchrotron (PS) to reach $26 \mathrm{GeV}$. These accelerated protons are then injected into the Super Proton Synchrotron (SPS). This accelerator has been the workhorse of many experiments since 1976, including its use as the source of $p \bar{p}$ collisions to the UA1 and UA2 experiments where the $W$ and $Z$ bosons were discovered. The SPS accelerates protons from the PS to $450 \mathrm{GeV}$ for injection into the LHC.

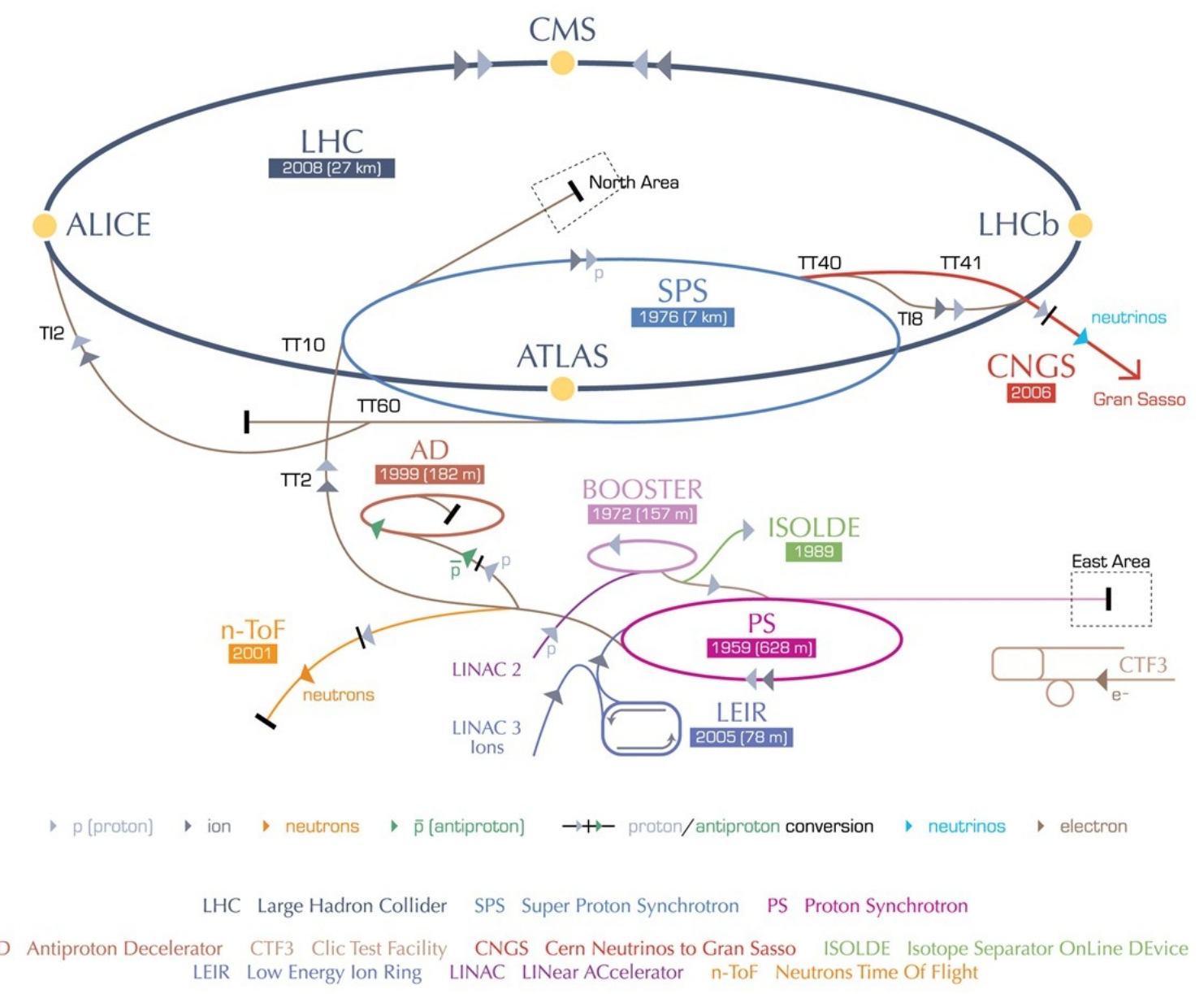

Figure 7.2: The accelerator complex at CERN, including the stages leading to the LHC. Various accelerators are labeled, along with their size and the year they were installed. The figure is not to scale. (c) 2008 CERN)

The Large Hadron Collider itself is $27 \mathrm{~km}$ in circumference and occupies a tunnel 
built approximately $100 \mathrm{~m}$ underground originally used to house the Large ElectronPositron Collider (LEP). The LHC was designed to accelerate two counter-circulating beams of protons to a design energy of $7 \mathrm{TeV}$ each with a design luminosity of $10^{34}$ $\mathrm{cm}^{-2} \mathrm{~s}^{-1}$. A series of 1232 dipole and 392 quadrupole NbTi superconducting magnets, cooled to approximately $1.9 \mathrm{~K}$ with liquid helium, steer and focus the beams for collisions at four interaction points along the ring. In September 2008 an electrical fault damaged several of the magnets and has forced the LHC to operate below design energy. From March 2010 until the end of 2011, the LHC operated with 3.5 $\mathrm{TeV}$ beams. This energy has been increased by $0.5 \mathrm{TeV}$ for collisions at $\sqrt{s}=8 \mathrm{TeV}$ during the 2012 data taking period.

\subsubsection{ATLAS}

The ATLAS detector can be divided into three principal groups of subdetectors. The Inner Detector consists of three specialized tracking systems inside a $2 \mathrm{~T}$ superconducting solenoidal magnetic field. The cryostat for this magnet is surrounded by a liquid argon electromagnetic calorimeter and a hadronic calorimeter made up of iron and scintillator material. A muon spectrometer consisting of several different tracking and scintillating modules constitutes the outermost group, embedded in an air-core toroidal magnetic field to provide independent measurements of muon momenta.

ATLAS utilizes a right-handed coordinate system with the origin at the center of the detector, the $z$-axis along the beam pipe, and the $x$-axis pointing from the interaction point toward the center of the LHC. The half of the detector in the $+z$ region is referred to as the "A side," and the $-z$ region as the "C side." 


\section{Inner Detector}

A series of three tracking detectors surround the interaction point to record the passage of charged particles. Two silicon tracking detectors provide precise measurements of track parameters and vertex reconstruction. Each subdetector consists of a barrel module with cylindrical symmetry along the $z$-axis and two endcap modules of tracking surfaces arranged in discs perpendicular to the $z$-axis. The barrel modules provide measurements along the $z$-axis and in the $r$ - $\phi$ plane, while the endcap modules resolve hits along the $r$ direction and in the $r-\phi$ plane.

The innermost subdetector, the Pixel detector, has approximately 80.4 million readout channels surrounding the beampipe, providing a hit resolution of $115 \mu \mathrm{m}$ along the $z$-axis and $10 \mu \mathrm{m}$ in the $r$ - $\phi$ plane perpendicular to the $z$-axis in the barrel module, and comparable resolution in the endcaps. The silicon microstrip tracker (SCT) consists of pairs of stereo silicon strips offset by $40 \mathrm{mrad}$ to measure space points along particle trajectories. Approximately 6.3 million such stereo strips provide measurement accuracies in the barrel of $580 \mu \mathrm{m}$ in the $z$ direction and $17 \mu \mathrm{m}$ in the $r$ - $\phi$ plane and comparable accuracies in the endcaps. Together the Pixel and SCT cover a region of $|\eta|<2.5$.

The Transition Radiation Tracker (TRT) is the outermost tracking detector of the ATLAS Inner Detector system. It consists of approximately 350,000 straw tubes, 4 $\mathrm{mm}$ in diameter, which act as proportional ionization counters. The TRT extends the ATLAS tracking capability in the region $|\eta|<2.0$ with typically 36 additional hits per track. When a charged particle passes through a straw, it leaves an ionization deposit in the xenon-based active gas mixture which drifts through an electric field toward the central wire. The drift time is recorded and translated into a corresponding 
drift radius with an accuracy of approximately $130 \mu \mathrm{m}$. Additionally, the straws are surrounded by a fibrous polymer filling (in the barrel) or layers of plastic foils (in the endcaps) in which electrons produce transition radiation photons with X-ray energies. These photons then interact with the active gas of a straw tube to produce a strong ionization signal. The presence of these transition radiation signals provide a powerful tool for electron identification.

Together the ATLAS Inner Detector systems provide a resolution of approximately $\sigma_{p_{T}} / p_{T}=0.05 \%$ and is sensitive to tracks with $p_{T}$ as low as $0.5 \mathrm{GeV}$. Primary and secondary vertices can be identified with high accuracy of just a few tens of $\mu \mathrm{m}$.

\section{Calorimeters}

Surrounding the cryostat housing the tracking solenoidal magnet is an electromagnetic (EM) calorimeter system. This system consists of finely-segmented sampling calorimeters covering the range $|\eta|<4.9$. Accordion-shaped lead absorber plates are immersed in active liquid argon (LAr) sensitive to electromagnetically interacting particles. The EM calorimeter measures the energy deposited when charged particles shower in the active volume and provides a resolution of $\sigma_{E} / E \approx 10 \%$. Measurements from a presampler in the region $|\eta|<1.8$ are used to correct the energy lost by electrons and photons prior to entering the calorimeter.

Hadronic calorimetry is provided in the barrel region by a detector consisting of alternating layers of steel absorber and scintillating tiles. The steel provides a dense material with a high hadronic interaction probability to capture the energy of particles such as protons, neutrons, and neutral pions. This calorimeter provides coverage out to $|\eta|=1.7$ and an energy resolution $\sigma_{E} / E \approx 50 \%$. The tile calorimeter 
is augmented by two LAr hadronic endcap calorimeters, similar to the EM calorimeter system, which extends coverage to $|\eta|=3.2$. Finally, the LAr forward calorimeter provides additional coverage at high eta which is robust against the large radiation exposure in the forward regions.

\section{Muon Spectrometer}

Large air core toroidal magnets provide a $4 \mathrm{~T}$ toroidal magnetic field, bending muons along the $z$-axis to provide an independent measurement of their momentum. Four muon detector subsystems are arrayed across the outer-most region of the ATLAS detector. Monitored Drift Tubes (MDT's) cover most of the muon acceptance and provide drift circle measurements in a similar manner to that of the TRT. At large $|\eta|$, additional multiwire proportional chambers called Cathode Strip Chambers (CSC's) extend muon acceptance with high granularity. Resistive Plate Chambers (RPC's) in the barrel region and Thin Gap Chambers (TGC's) in the endcaps deliver fast particle tracking for the trigger system and contribute additional tracking information for event reconstruction. All together the muon spectrometer provides a momentum resolution $\sigma_{p_{T}} / p_{T} \approx 10 \%$ at $p_{T}=1 \mathrm{TeV}$ (independent of the Inner Detector).

\section{Trigger and Data Acquisition}

A three-tier trigger system reduces the rate at which events are recorded from the delivered collision rate of approximately $40 \mathrm{MHz}$ down to about $200 \mathrm{~Hz}$. The hardwarebased Level-1 (L1) trigger system first reduces the rate to $\approx 75 \mathrm{kHz}$ (limited by the bandwidth of the readout system). The high-level trigger is software-based and consists of the Level-2 (L2) trigger, based on fast reconstruction algorithms, followed by 
full reconstruction across a trigger farm in the event filter.

The large amount of data acquired by the ATLAS experiment puts high demands on storage facilities. To process the recorded data and make it available for analysis by the numerous collaboration members, a sophisticated grid computing paradigm has been adopted. Data sets are duplicated and stored at various computing centers around the world. Most preliminary analysis tasks, as well as Monte Carlo simulation, are performed remotely on the computing clusters at these centers.

\subsection{Expected signature}

As with the analysis at the $\mathrm{D} \emptyset$ experiment, we expect the most obvious signature at ATLAS of Lorentz violation in the top quark sector to manifest as a sidereal modulation in the $t \bar{t}$ event rate. We can construct an expectation for the time dependent $t \bar{t}$ cross section using the same machinery employed previously. It still has the form $\sigma(t)=\sigma_{\mathrm{SM}} \mathcal{B} \epsilon\left(1+f_{\mathrm{SME}}(t)\right)$, with the same forms for each possible $f_{\mathrm{SME}}(t)$ but different amplitudes and phases. This difference is due to three primary factors - the orientation and location of the ATLAS experiment, the kinematic phase space acces-

sible to the $t \bar{t}$ system at the higher collision energy, and the dominance of the $g g \rightarrow t \bar{t}$ production process in $p p$ collisions at the LHC over $q \bar{q} \rightarrow t \bar{t}$ (which was the dominant process at the Tevatron).

\subsubsection{Detector location and orientation}

ATLAS is located at the interaction point along the LHC nearest to the main CERN laboratory campus, designated "Point 1." It lies approximately 100 meters under- 
ground, below a point on the Franco-Swiss border about $8 \mathrm{~km}$ northwest the city of Geneva, Switzerland. The colatitude of this location $\chi=43.76^{\circ}$, slightly further north than the $\mathrm{D} \emptyset$ detector. The $z$-axis of the ATLAS reference frame points along the clockwise-circulating proton beam, at an angle of about $11^{\circ}$ south of east. Similar to the case for the $\mathrm{D} \varnothing$ analysis, the transformation $R_{\mu}^{\alpha}(t)$ is the product of a rotation about the detector $y$-axis by $\alpha \approx 101^{\circ}$, followed by a rotation about the $x$-axis by $\pi / 2$. A second rotation about the $y$-axis by $\chi$ aligns the $z$-axis with that of the Sun-centered reference frame. Finally, a rotation about this $Z$-axis by an angle of $\omega t$ introduces time dependence at the sidereal frequency $\omega$. Again we have

$$
R(t)=\tilde{R}_{z}(\omega t) \tilde{R}_{y}(\chi) \tilde{R}_{x}(\pi / 2) \tilde{R}_{y}(\alpha)
$$

using the appropriate rotation parameters for the ATLAS reference frame relative to the Sun-centered reference frame with the rotation matrices defined in Eqs. (6.7)(6.9).

The location and orientation of the ATLAS detector mean it is potentially more sensitive to SME coefficients coupling to the $X-Y$ plane (i.e. coefficients of the form $C_{X X}, C_{X Y}, C_{X Z}$, and $C_{Y Z}$ ) compared to DØ. The ATLAS collision axis points more directly into this plane ( $\alpha$ closer to 0 or $\pi$ ). A smaller deviation of the beam axis from the $X-Y$ plane enhances the contribution from the already large $A^{z z}$ element. Comparing the amplitudes of the expected sidereal contribution from the terms $R_{\mu}^{\alpha}(t)$, $A_{P}^{\alpha \beta}$, and $A_{F}^{\alpha \beta}$ in Eqs. (6.21)-(6.24) between the two experiments, we find that while these terms contribute a similar magnitude to the $X X$ and $X Y$ cases, the contribution for the $X Z$ and $Y Z$ cases is larger at ATLAS than at $\mathrm{D} \varnothing$. The exact increase depends on the choice of SME coefficients to extract and the values of $A_{P}^{\alpha \beta}$ and $A_{F}^{\alpha \beta}$ at the two 
detectors. Qualitatively, these larger contributions to the amplitude of $f_{\mathrm{SME}}(t)$ for the latter two cases will increase the amplitude of sidereal time dependence due to the orientation of the detector and tend to increase the sensitivity to SME coefficients.

\subsection{2 $A_{P}^{\alpha \beta}$ and $A_{F}^{\alpha \beta}$ contributions}

There are two key differences between the production process for $t \bar{t}$ events at ATLAS versus $\mathrm{D} \emptyset$ that will affect the expression for $f_{\mathrm{SME}}(t)$ and the resulting sensitivity of the analysis. The initial state processes which produce the $t \bar{t}$ system differ in $p p$ collisions versus $p \bar{p}$, and the distributions of parton momenta in the $t \bar{t}$ system will change with the collision energy. These differences will alter the sensitivity of ATLAS to SME coefficients through the terms $A_{P}^{\alpha \beta}$ and $A_{F}^{\alpha \beta}$ derived from average event kinematics.

Because the production mechanism for $t \bar{t}$ events at $\mathrm{D} \varnothing$ was through the $p \bar{p}$ initial state, where a proton collided with an antiproton, the initial partons producing the $t \bar{t}$ system were dominantly an interacting quark and antiquark pair. This initial state constituted $\approx 85 \%$ of $t \bar{t}$ interactions, while the remaining $\approx 15 \%$ of events came from gluon fusion [26]. The expression for the modified matrix element describing $t \bar{t}$ production and decay, Eqs. (3.2)-(3.9), was derived using only diagrams for the process $q \bar{q} \rightarrow t \bar{t}$, dominant at the Tevatron. The fractions of events proceeding through $q \bar{q}$ initial states and $g g$ initial states are essentially reversed in proton-proton collisions at the LHC. The matrix element describing $t \bar{t}$ production proceeding via gluon fusion will better describe the effects of SME coefficients on the $t \bar{t}$ production rate at ATLAS. However, because of the increased complexity and number of contributing processes this matrix element has not yet been derived. Instead, we will develop our 
prospectus based upon the matrix element already presented. The specific values of each component of $A_{P}^{\alpha \beta}$ and $A_{F}^{\alpha \beta}$ may be affected by the difference between these processes, but the analysis method will be independent of this difference.

The other principal difference between $t \bar{t}$ production at ATLAS versus D $\varnothing$ that will affect the sensitivity of the analysis is the distribution of parton four-momenta in $t \bar{t}$ events created at the two different collision energies. A qualitative expectation of this difference can be inferred by considering the event-by-event contributions to the elements of $A_{P}^{\alpha \beta}$ and $A_{F}^{\alpha \beta}$ in Figs. B.1-B.4 of Appendix B. Because of the higher collision energies, we expect each distribution to broaden as the upper limit of transverse and longitudinal parton momenta, as well as total parton energies, increases. The averages of all off-diagonal elements will likely remain stable at zero, since both detectors have the same basic geometry (azimuthal symmetry and symmetric $\eta$ coverage). However, the means of the on-diagonal elements will most certainly increase. This will increase the expected amplitude of $f_{\mathrm{SME}}(t)$ through the contributions to the $b$ terms of Eqs. (6.18)-(6.20) and (6.25)-(6.26), thereby increasing sensitivity to the SME coefficients. A recalculation of the elements of $A_{P}^{\alpha \beta}$ and $A_{F}^{\alpha \beta}$ using simulated Monte Carlo $t \bar{t}$ data at LHC collision conditions will yield quantitative values for this increase. For this discussion we estimate the sensitivity of ATLAS to SME coefficients by using the same values of $A_{P}^{\alpha \beta}$ and $A_{F}^{\alpha \beta}$ calculated for the $\mathrm{D} \varnothing$ analysis, with the expectation that our estimate will likely be conservative. 


\subsection{Event selection}

Several analyses have already examined top production and properties at ATLAS. A number of measurements of the $t \bar{t}$ production cross section have been made using integrated luminosities from $35 \mathrm{pb}^{-1}$ up to $2.05 \mathrm{fb}^{-1}$ [44]. Figure 7.3 compares the measurements in various channels. The best combination reported so far measured $\sigma_{t \bar{t}}=177 \pm 3$ (stat.) ${ }_{-7}^{+8}$ (syst.) \pm 7 (lumi.) pb [41], in good agreement with the Standard Model expectation. A $1.04 \mathrm{fb}^{-1}$ sample of events in the lepton plus jets channel was used to measure the top quark mass, determining $m_{\text {top }}=174.5 \pm 0.6$ (stat.) \pm 2.3 (syst.) $\mathrm{GeV}$ [45]. A search for $t \bar{t}$ resonant states has even been conducted, limiting the possible mass of such a resonance to $m_{Z^{\prime}}<880 \mathrm{GeV}$ for a leptophobic topcolour $Z^{\prime}$ boson and $m_{g_{\mathrm{KK}}}<1130 \mathrm{GeV}$ in the case of a Kaluza-Klein gluon excitation within the Randall-Sundrum model of warped extra dimensions [46]. 


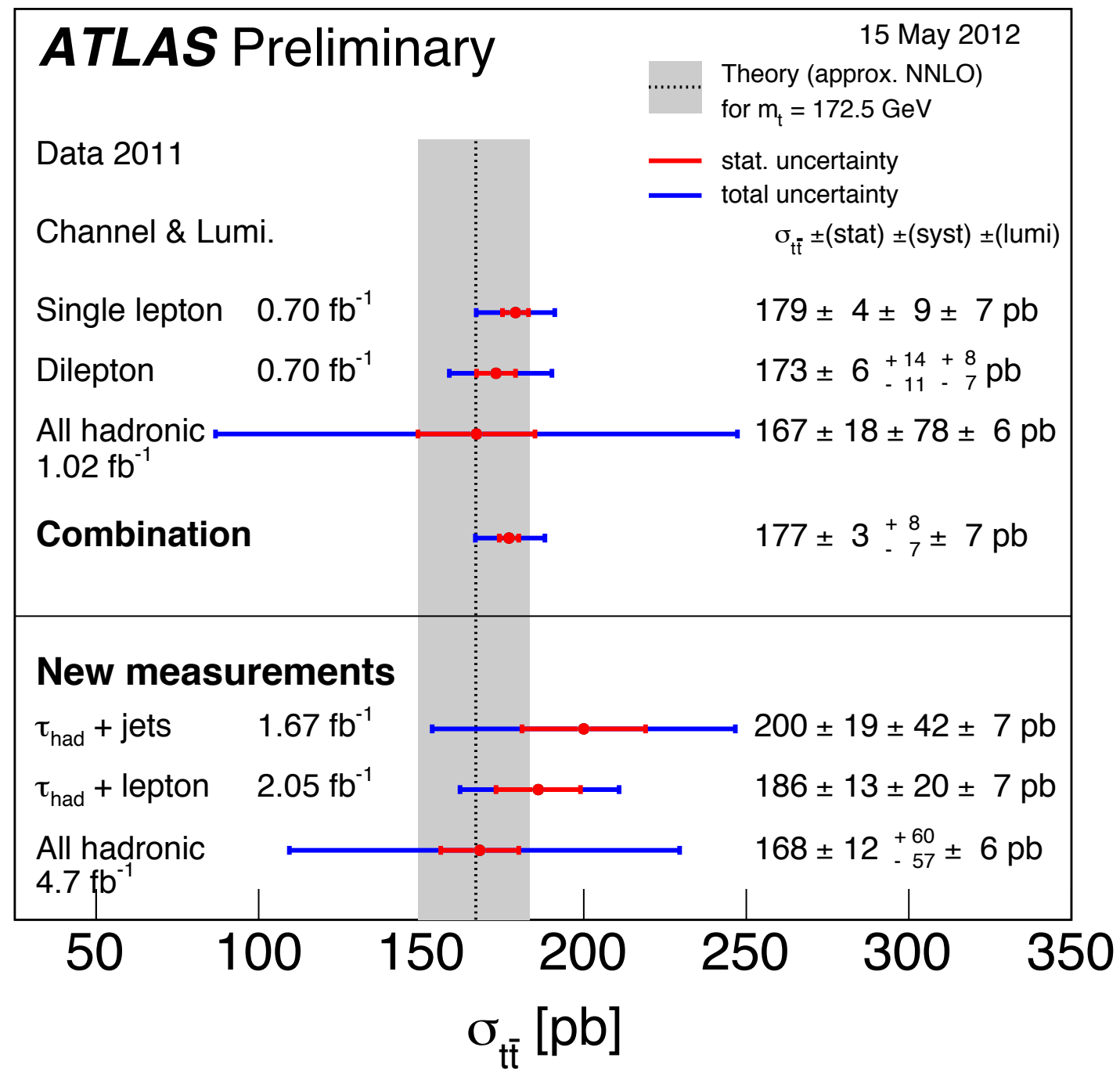

Figure 7.3: Summary of measurements of the $t \bar{t}$ production cross section compared to the corresponding theoretical expectation. The upper part of the figure shows measurements that are averaged to give the combined value shown. The lower part shows additional newer measurements not included in the combination. (ATLAS Experiment (c) 2012 CERN) 
Of the published analyses studying top quark properties at ATLAS, the latter analysis - the search for a $t \bar{t}$ resonance using $2.05 \mathrm{fb}^{-1}$ of data at $\sqrt{s}=7 \mathrm{TeV}$ - could most easily be extended to perform a search for Lorentz violation in the top sector with the $t \bar{t}$ system using similar techniques to those of the $\mathrm{D} \emptyset$ search. This analysis examined the largest data set so far, and its event selection criteria yielded the largest $t \bar{t}$ signal fraction (approximately $79 \%$ ). We consider the single-lepton event selection, in which a total of $9622 e+$ jets events and $12706 \mu+$ jets events comprised the signal sample. Simple single-lepton triggers were chosen, with a minimum $p_{T}$ of $20 \mathrm{GeV}$ or $22 \mathrm{GeV}$ for the electron selection and $18 \mathrm{GeV}$ for $\mu+$ jets. Events were then required to be reconstructed with at least one primary vertex containing at least five tracks with $p_{T}>0.4 \mathrm{GeV}$. There must be exactly one isolated lepton (electron or muon) in the event, and restrictions were placed on the properties of non-isolated leptons. Minimum $\mathscr{H}_{T}$ and transverse mass requirements helped enhance the $t \bar{t}$ event fraction in each channel. Selected events contained either at least four jets with $p_{T}>25 \mathrm{GeV}$ and $|\eta|<2.5$ or three such jets if one has an invariant mass of at least $60 \mathrm{GeV}$; the latter case is typical for $t \bar{t}$ events in which one of the top quarks is produced with a sufficient boost factor that its decay products are collimated (approximately $0.3 \%$ of events). The leading jet was required to have $p_{T}>60 \mathrm{GeV}$ and at least one jet in the event was tagged as a $b$-jet.

Background and signal expectations were modeled using various Monte Carlo generators and a sophisticated simulation of the ATLAS detector. In this event selection, approximately $79 \%$ of events are actual $t \bar{t}$ production and decay, while $W+$ jets production constitutes the majority of the background. The contributions from various processes to the total number of events are reproduced from Ref. [46] in 
Table 7.1.

Table 7.1: Comparison of yields for the $e+$ jets and $\mu+$ jets channels for the $t \bar{t}$ resonance study to contributions expected from $t \bar{t}$ signal and sources of background. The uncertainties given are due to systematic effects, while statistical uncertainties are small and neglected.

\begin{tabular}{lcc}
\hline \hline Contribution & $e+$ jets & $\mu+$ jets \\
\hline$t \bar{t}$ & $7830 \pm 750$ & $10000 \pm 960$ \\
Single top & $470 \pm 50$ & $570 \pm 60$ \\
$W+$ jets & $1120 \pm 540$ & $1450 \pm 700$ \\
$Z+$ jets & $85 \pm 40$ & $90 \pm 45$ \\
Diboson & $18 \pm 1$ & $18 \pm 1$ \\
Multijet & $340 \pm 170$ & $470 \pm 240$ \\
\hline Total Expected & $9860 \pm 940$ & $12600 \pm 1210$ \\
Total Observed & 9622 & 12706 \\
\hline \hline
\end{tabular}

A search for Lorentz violation in $t \bar{t}$ production and decay using this analysis selection has a comparable signal purity to that of the $\mathrm{D} \varnothing$ analysis $\left(f_{S} \approx 0.79\right)$ but an increase in statistics by about a factor of 30 .

The $t \bar{t}$ resonance analysis did not report a detailed study of event selections with large contributions from background (non- $t \bar{t}$ ) events. These selections were useful to the $\mathrm{D} \varnothing$ analysis as a cross-check to confirm the lack of unexpected sidereal time dependence induced by non-LV effects, e.g. 24-hour periodic variations in detector efficiencies. However, the shorter run period of the ATLAS data set suggests a different method for cross-checks would be most appropriate. This will be discussed later. 


\subsection{Expected sensitivity}

The statistical uncertainty will likely still be the largest contribution to the limits on SME coefficients measurable in $t \bar{t}$ production and decay at ATLAS. The dominant improvement that ATLAS can provide over the DØ results therefore comes through the larger sample size. Table 7.2 compares the number of events in the signal event selection expected at ATLAS described above to that used for the analysis at D $\varnothing$.

Table 7.2: Comparison of signal sample size for the $e+$ jets and $\mu+$ jets channels for the $t \bar{t}$ resonance study to those used for the $\mathrm{D} \varnothing$ analysis.

\begin{tabular}{c|cc}
\hline \hline & ATLAS & DØ \\
\hline$e+$ jets & 9622 & 401 \\
$\mu+$ jets & 12706 & 345 \\
\hline \hline
\end{tabular}

The $e+$ jets sample is larger by a factor of 24 and the $\mu+$ jets sample is larger by a factor of 37. Assuming conservatively that the contributions from the detector location and orientation, as well as those from $A_{P}^{\alpha \beta}$ and $A_{F}^{\alpha \beta}$, to the expected sidereal amplitude will be comparable to those determined for the $\mathrm{D} \varnothing$ analysis, we can scale the statistical uncertainties of Tables $6.15-6.22$ by the square root of the increase in sample size to determine the expected statistical sensitivity of ATLAS. Compared to the $\mathrm{D} \varnothing$ analysis, statistical uncertainties are reduced by a factor of 4.9 for the $e+$ jets sample and by a factor of 6 for the $\mu+$ jets sample. Tables $7.3-7.6$ compare this expectation to that found in the $\mathrm{D} \varnothing$ analysis. ATLAS has the potential for nearly an order of magnitude in improvement on the limits set by DØ in Ref. [1] and may be able to set the first limits on the linear combination $c_{\mu \nu}=\left(c_{L}\right)_{\mu \nu}+\left(c_{R}\right)_{\mu \nu}$. 
Table 7.3: The expected statistical uncertainty on $\left(c_{L}\right)_{\mu \nu}$ at ATLAS compared to that of the $\mathrm{D} \varnothing$ analysis.

\begin{tabular}{c|cc|cc}
\hline \hline & \multicolumn{2}{|c}{$e+$ jets } & \multicolumn{2}{c}{$\mu+$ jets } \\
\hline Coefficient & ATLAS & D & ATLAS & D \\
\hline$\left(c_{L}\right)_{X X}$ & 0.036 & 0.178 & 0.023 & 0.141 \\
$\left(c_{L}\right)_{X Y}$ & 0.037 & 0.179 & 0.023 & 0.142 \\
$\left(c_{L}\right)_{X Z}$ & 0.024 & 0.120 & 0.016 & 0.097 \\
$\left(c_{L}\right)_{Y Z}$ & 0.025 & 0.122 & 0.016 & 0.099 \\
\hline \hline
\end{tabular}

Table 7.4: The expected statistical uncertainty on $\left(c_{R}\right)_{\mu \nu}$ at ATLAS compared to that of the $\mathrm{D} \varnothing$ analysis.

\begin{tabular}{c|cc|cc}
\hline \hline & \multicolumn{2}{|c}{$e+$ jets } & \multicolumn{2}{c}{$\mu+$ jets } \\
\hline Coefficient & ATLAS & D & ATLAS & D \\
\hline$\left(c_{R}\right)_{X X}$ & 0.024 & 0.119 & 0.022 & 0.131 \\
$\left(c_{R}\right)_{X Y}$ & 0.024 & 0.120 & 0.022 & 0.132 \\
$\left(c_{R}\right)_{X Z}$ & 0.019 & 0.091 & 0.017 & 0.100 \\
$\left(c_{R}\right)_{Y Z}$ & 0.019 & 0.092 & 0.017 & 0.101 \\
\hline \hline
\end{tabular}

Table 7.5: The expected statistical uncertainty on $c_{\mu \nu}$ at ATLAS compared to that of the $\mathrm{D} \varnothing$ analysis.

\begin{tabular}{c|cc|cc}
\hline \hline & \multicolumn{2}{|c}{$e+$ jets } & \multicolumn{2}{c}{$\mu+$ jets } \\
\hline Coefficient & ATLAS & D & ATLAS & D \\
\hline$c_{X X}$ & 0.148 & 0.727 & 0.622 & 3.769 \\
$c_{X Y}$ & 0.149 & 0.732 & 0.627 & 3.801 \\
$c_{X Z}$ & 0.138 & 0.674 & 0.264 & 1.599 \\
$c_{Y Z}$ & 0.135 & 0.660 & 0.266 & 1.609 \\
\hline \hline
\end{tabular}

Table 7.6: The expected statistical uncertainty on $d_{\mu \nu}$ at ATLAS compared to that of the $\mathrm{D} \varnothing$ analysis.

\begin{tabular}{c|cc|cc}
\hline \hline & \multicolumn{2}{|c}{$e+$ jets } & \multicolumn{2}{c}{$\mu+$ jets } \\
\hline Coefficient & ATLAS & D & ATLAS & D \\
\hline$d_{X X}$ & 0.029 & 0.142 & 0.022 & 0.136 \\
$d_{X Y}$ & 0.029 & 0.143 & 0.023 & 0.137 \\
$d_{X Z}$ & 0.021 & 0.104 & 0.016 & 0.099 \\
$d_{Y Z}$ & 0.021 & 0.105 & 0.017 & 0.100 \\
\hline \hline
\end{tabular}




\subsubsection{Power spectrum of the event rate}

As discussed previously, the $t \bar{t}$ resonance analysis performed by ATLAS only studied the signal event selection of events with a lepton plus at least 4 jets. A study of the events in this selection with fewer jets, likely dominated by $W+$ jets production, was not reported. These events are therefore will likely not be immediately available for the same cross-check procedure employed by the $\mathrm{D} \emptyset$ analysis. Furthermore, the period of time over which the ATLAS data was collected was significantly shorter than that of the $\mathrm{D} \varnothing$ analysis. The dilution of any potential solar (24-hour) periodic contribution due to variations in detector efficiencies is therefore not guaranteed to be as significant. A different method of controlling for non-sidereal periodic effects is therefore necessary.

By utilizing the data describing the full instantaneous luminosity profile for the run period covering the event selection, we can estimate the amplitude of any period oscillation in the luminosity corrected event rate at any frequency. The power spectrum corresponding to this rate can be approximated using a Lomb periodogram. This tool approximates a Fourier transform of time dependent data which is collected at non-uniform intervals. The method is described in detail in Ref. [47], and Ref. [20] provides an example of its use. The spectral power at a frequency $\omega$ is

$$
P(\omega) \equiv \frac{\left|\sum_{j=1}^{N} r_{j} e^{2 i \pi \omega t_{j}}\right|^{2}}{N \sigma^{2}\left(r_{j}\right)} .
$$

For our analysis, the $N$ measurements at times $t_{j}$ correspond to the luminosity blocks from which candidate $t \bar{t}$ events are drawn by the event selections. The measurements $r_{j}$ are the luminosity-corrected event rates at those times, such that 


$$
r_{j} \approx \frac{n_{j}^{(t \bar{t})} / \Delta t_{j}}{\mathcal{L}_{j} / \mathcal{L}_{\text {int }}}
$$

where $n_{j}^{(t t)}$ is the number of candidate $t \bar{t}$ events in luminosity block $j$ (typically 0 or 1), $\Delta t_{j}$ is the width of the luminosity block (typically $60 \mathrm{~s}$ ), and $\mathcal{L}_{j}$ is the integrated luminosity collected during that luminosity block. The probability that $P(\omega)$ exceeds a value $S$ in the absence of a time dependence in the event rate at a given frequency (where events occur at random times due to the probabilistic production process) is an exponential function $\exp (-S)$. If we test $M$ independent frequencies, the probability that $P(\omega)$ exceeds $S$ is $1-[1-\exp (-S)]^{M}$. This provides a handle on the significance of a peak in the power spectrum at any given frequency.

Following the example of the BaBar search for CPT and Lorentz violation in $B^{0}-\bar{B}^{0}$ oscillations [20], by testing over 20,000 frequencies we would oversample the frequency range by a factor of about 2.2 and avoid underestimating the spectral power of signals at the tested frequencies. This corresponds to about 9500 independent frequencies and provides sufficient frequency resolution to resolve both sidereal and solar periods. As an example of this procedure in practice, Fig. 7.4 reproduces the power spectrum derived in the BaBar search for CPT and Lorentz violation in $B^{0}-\bar{B}^{0}$ oscillations [20]. Since LV in $t \bar{t}$ events could also manifest at a frequency of one or two oscillations per sidereal day, our analysis would need to examine frequencies corresponding to a comparable range. 


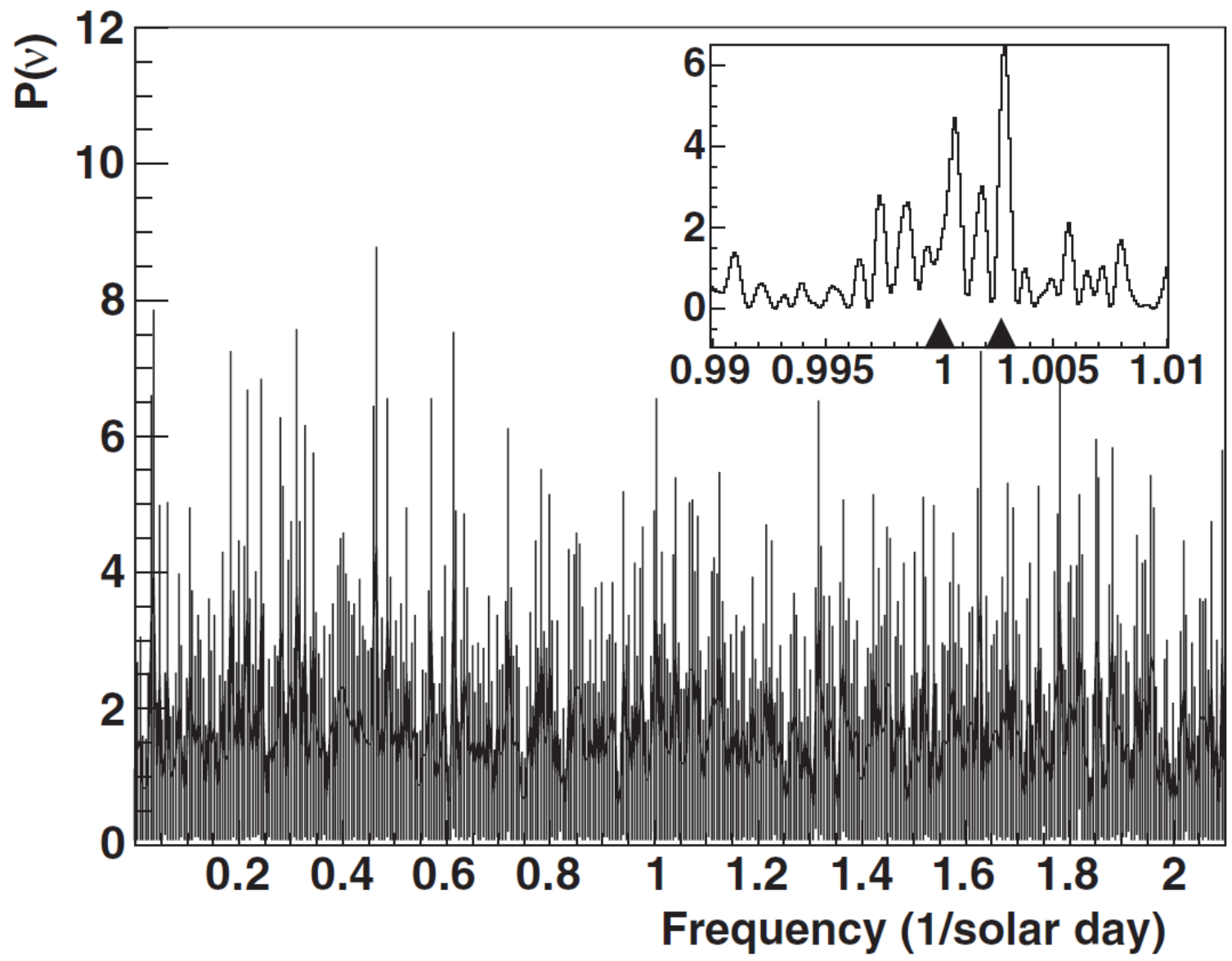

Figure 7.4: The Periodogram used by the BaBar experiment to search for signs of CPT and Lorentz violation through variations in $B^{0}-\bar{B}^{0}$ oscillations observed through opposite-sign dilepton events in $\Upsilon(4 S) \rightarrow B \bar{B}$ decays [20]. The triangles in the inset indicate the solar and sidereal frequencies. No significant signal appears at any frequency. 


\subsection{Likelihood analysis}

The robustness of the analysis can be improved if the full profile of the instantaneous luminosity corresponding to the signal data set is available. While the integrated luminosity in bins of sidereal phase was sufficient for the $\mathrm{D} \varnothing$ analysis, with the full instantaneous luminosity profile we could perform a more sensitive analysis using a maximum likelihood technique.

For each event, we consider the joint probability of the observed data (the time of the event, $t$ ) and the SME coefficient to be extracted, $C$. The likelihood function is defined as the product of this probability for each event in the sample. This probability depends on the event type - signal $(t \bar{t})$ or background (non- $t \bar{t})$. The per-event probability is

$$
\begin{aligned}
P_{\text {evt }}(t, C) & =\sum_{\text {type }} P_{\text {evt }}(t, C \text {, type }) \\
& =\sum_{\text {type }} P(t \mid \text { type, } C) P(\text { type }, C) \\
& =\sum_{\text {type }} P(t \mid \text { type, } C) P(\text { type }) P(C) \\
& =\left[P_{\text {sig }}(t \mid C) P(\text { type }=\operatorname{sig})+P_{b k g}(t) P(\text { type }=\mathrm{bkg})\right] P(C) .
\end{aligned}
$$

We treat the signal probability $P_{\text {sig }}(t \mid C)$ as the instantaneous event rate, $\sigma(t) \mathcal{L}(t)$, normalized to the event rate integrated over the data taking period $\tau$ : 


$$
\begin{aligned}
P_{\text {sig }}(t \mid C) & =\frac{\sigma(t) \mathcal{L}(t)}{\int_{\tau} \sigma(t) \mathcal{L}(t) d t} \\
& \approx \frac{1}{\tau} \frac{\mathcal{L}(t)}{\mathcal{L}_{\text {int }}}\left(1+f_{\mathrm{SME}}(t)\right) .
\end{aligned}
$$

$P($ type $=\operatorname{sig})$ is the average signal fraction, $f_{S}$. The background probability is again assumed to be proportional only to the instantaneous luminosity such that $P_{b k g}(t)=$ $1 / \tau \times \mathcal{L}(t) / \mathcal{L}_{\text {int }}$ and the background fraction $P($ type $=\mathrm{bkg})=1-f_{S}$. Finally, the prior probability density for the SME coefficients, $P(C)$, is assumed to be flat and therefore does not contribute to the maximization of the likelihood function; nor does the constant $1 / \tau$.

To extract values for the SME coefficients from the data, we maximize the function

$$
\begin{aligned}
L(C) & =\prod_{\text {evt }} P_{\text {evt }}(t, C) \\
& =\prod_{\text {evt }} \frac{\mathcal{L}(t)}{\mathcal{L}_{\text {int }}}\left(f_{S} P_{\text {sig }}(t \mid C)+\left(1-f_{S}\right)\right) \\
& =\prod_{\text {evt }} \frac{\mathcal{L}(t)}{\mathcal{L}_{\text {int }}}\left(1+f_{S} f_{\mathrm{SME}}(t)\right) .
\end{aligned}
$$

The values of the SME coefficients at the maximum of this function [in practice, the minimum of $-\ln (L)]$ represents those values which best describe the observed data.

We could also expand these probability density functions to include a possible contribution from detector effects exhibiting a periodic dependence on solar (24-hour) time with amplitude $A_{\text {sol }}$ and phase $\phi_{\text {sol }}$. Integrating over this phase from $\phi_{\text {sol }}=0$ to $\phi_{\text {sol }}=\pi$ (with constant prior probability $P\left(\phi_{\text {sol }}\right)$ ), the signal and background 
probabilities would become

$$
\begin{aligned}
P_{\text {sig }}\left(t \mid C, A_{\mathrm{sol}}\right) & \approx \frac{1}{\tau} \frac{\mathcal{L}(t)}{\mathcal{L}_{\mathrm{int}}}\left(1+f_{\mathrm{SME}}(t)+2 A_{\mathrm{sol}} \cos \left(\omega_{\mathrm{sol}} t\right)\right), \\
P_{\text {bkg }}\left(t, A_{\mathrm{sol}}\right) & \approx \frac{1}{\tau} \frac{\mathcal{L}(t)}{\mathcal{L}_{\mathrm{int}}}\left(1+2 A_{\mathrm{sol}} \cos \left(\omega_{\mathrm{sol}} t\right)\right) .
\end{aligned}
$$

Equation (7.8) would become a two-dimensional likelihood function dependent on both $C$ and $A_{\text {sol }}$ such that

$$
L\left(C, A_{\mathrm{sol}}\right)=\prod_{\text {evt }} \frac{\mathcal{L}(t)}{\mathcal{L}_{\text {int }}}\left(1+f_{S} f_{\mathrm{SME}}(t)+2 A_{\mathrm{sol}} \cos \left(\omega_{\mathrm{sol}} t\right)\right) .
$$

The neighborhood of the maximum of this likelihood function would clearly quantify the relationship between any possible periodic signals at both the sidereal and solar frequencies.

\subsection{Further possibilities}

The reach of a search for Lorentz violation in top quark production and decay at ATLAS could potentially be extended beyond what has been outlined here. As the detector continues to collect data, the sample size of events containing top quarks will increase substantially. Larger $t \bar{t}$ data sets could be examined in bins of pseudorapidity or azimuthal angle while still maintaining sufficient statistical sensitivity to draw meaningful conclusions. The most prominent signal of Lorentz violation would likely still be a time-varying dependence on the direction of top quarks and their decay products with respect to the Sun-centered reference frame. Contributions to some of the off-diagonal elements of $A_{P}^{\alpha \beta}$ and $A_{F}^{\alpha \beta}$ may not average to zero when considered in 
bins of e.g. $\eta$ or $\phi$, and an analysis using such segmented data sets may be sensitive to additional SME coefficients coupling to those off-diagonal elements.

Additionally, the cross section for producing events containing a single top quark is much higher at the LHC than at the Tevatron. This analysis has only considered $t \bar{t}$ production and decay, but there is the potential for other SME coefficients to make significant contributions to the production of single $t$ and $\bar{t}$ quarks. ATLAS is accumulating a large collection of single top quark candidate events [48]. An analysis of single top events may also be sensitive to CPT-violating effects by comparing the rates of the two processes.

Regardless of the magnitude of the gains from increasingly sophisticated versions of the analysis to search for Lorentz violation in top quark events, the ATLAS experiment is positioned to make substantial improvements to the limits already set on the top sector SME coefficients by virtue of its statistical power alone. ATLAS has the potential for an order of magnitude improvement on the existing limits, and may be able to provide even more sensitivity. 


\section{Chapter 8}

\section{Conclusions}

We have performed the first search for signals of Lorentz violation in the top quark sector by examining $t \bar{t}$ pair production and decay in data collected with the $D \varnothing$ particle detector at the Fermilab Tevatron accelerator. Violation of Lorentz symmetry would most prominently affect this process by introducing a unique periodic time dependence in the $t \bar{t}$ cross section. The corresponding event rate would exhibit a modulation as a function of sidereal time as the detector reference frame rotates with the Earth relative to the Sun-centered reference frame. To maximize sensitivity to this time dependence, we considered events containing a single isolated lepton plus at least three jets, where the lepton is either an electron or a muon. The non-t $\bar{t}$ backgrounds in this selection are principally from kinematically similar events, like $W+$ jets production, and dominate lepton plus jets events in which only two or three jets are observed. We were able to take advantage of these latter event selections as cross-checks to confirm that no unexpected periodic time dependence, such as day/night temperature cycling or accelerator conditions, affected our conclusions. We 
found that both the signal- and background-dominated event selections are consistent with no sidereal time dependence.

The Standard-Model Extension is a phenomenological framework that provides a mathematical description of the contributions of general Lorentz- and CPT-violating operators that can be added to the Lagrange density of the Standard Model. In this way, the SME allows us to develop and test a quantitative expectation for how such contributions effect observables like the $t \bar{t}$ cross section. We translated the contribution from the SME coefficients $\left(c_{L}\right)_{\mu \nu}=\left(c_{Q}\right)_{\mu \nu 33}$ and $\left(c_{R}\right)_{\mu \nu}=\left(c_{U}\right)_{\mu \nu 33}$ to the matrix element describing $t \bar{t}$ production and decay via the process $q \bar{q} \rightarrow t \bar{t}$ into an expectation for the $t \bar{t}$ event rate, and compared this to data from the $\mathrm{D} \varnothing$ detector.

This analysis is sensitive to the $X X, Y Y, X Y, X Z$, and $Y Z$ components of $\left(c_{L}\right)_{\mu \nu}$ and $\left(c_{R}\right)_{\mu \nu}$, as well as the linear combinations $c_{\mu \nu}=\left(c_{L}\right)_{\mu \nu}+\left(c_{R}\right)_{\mu \nu}$ and $d_{\mu \nu}=\left(c_{L}\right)_{\mu \nu}-\left(c_{R}\right)_{\mu \nu}$. We find these coefficients to be consistent with zero within two standard deviations. We define the observed limits for each coefficient as the extracted value \pm 2 standard deviations, corresponding to $95 \%$ confidence level intervals. Because these intervals are larger than unity for the linear combination $c_{\mu \nu}$, we do not report measurements or limits for those coefficients. The central values, statistical and systematic unceratinties, and corresponding C.L. intervals for the remaining coefficients are presented in Tables 8.1-8.3.

We have also considered how this analysis might be performed at the ATLAS detector using high energy proton-proton collisions delivered by the Large Hadron Collider. The $t \bar{t}$ production cross section is much higher at this experiment and the statistical power of a comparable analysis is correspondingly greater compared to the DØ analysis. The higher energy of these $p p$ collisions, as well as the location and 
Table 8.1: Limits on SME coefficients at the 95\% C.L., assuming $\left(c_{U}\right)_{\mu \nu} \equiv 0$.

\begin{tabular}{crc}
\hline \hline Coefficient & Value \pm Stat. \pm Sys. & $95 \%$ C.L. Interval \\
\hline$\left(c_{Q}\right)_{X X 33}$ & $-0.12 \pm 0.11 \pm 0.02$ & {$[-0.34,+0.11]$} \\
$\left(c_{Q}\right)_{Y Y 33}$ & $0.12 \pm 0.11 \pm 0.02$ & {$[-0.11,+0.34]$} \\
$\left(c_{Q}\right)_{X Y 33}$ & $-0.04 \pm 0.11 \pm 0.01$ & {$[-0.26,+0.18]$} \\
$\left(c_{Q}\right)_{X Z 33}$ & $0.15 \pm 0.08 \pm 0.02$ & {$[-0.01,+0.31]$} \\
$\left(c_{Q}\right)_{Y Z 33}$ & $-0.03 \pm 0.08 \pm 0.01$ & {$[-0.19,+0.12]$} \\
\hline \hline
\end{tabular}

Table 8.2: Limits on SME coefficients at the 95\% C.L., assuming $\left(c_{Q}\right)_{\mu \nu} \equiv 0$.

\begin{tabular}{crc}
\hline \hline Coefficient & Value \pm Stat. \pm Sys. & $95 \%$ C.L. Interval \\
\hline$\left(c_{U}\right)_{X X 33}$ & $0.10 \pm 0.09 \pm 0.02$ & {$[-0.08,+0.27]$} \\
$\left(c_{U}\right)_{Y Y 33}$ & $-0.10 \pm 0.09 \pm 0.02$ & {$[-0.27,+0.08]$} \\
$\left(c_{U}\right)_{X Y 33}$ & $0.04 \pm 0.09 \pm 0.01$ & {$[-0.14,+0.22]$} \\
$\left(c_{U}\right)_{X Z 33}$ & $-0.14 \pm 0.07 \pm 0.02$ & {$[-0.28,+0.01]$} \\
$\left(c_{U}\right)_{Y Z 33}$ & $0.01 \pm 0.07 \pm<0.01$ & {$[-0.13,+0.14]$} \\
\hline \hline
\end{tabular}

Table 8.3: Limits on SME coefficients at the 95\% C.L., assuming $c_{\mu \nu} \equiv 0$.

\begin{tabular}{crc}
\hline \hline Coefficient & Value \pm Stat. \pm Sys. & $95 \%$ C.L. Interval \\
\hline$d_{X X}$ & $-0.11 \pm 0.10 \pm 0.02$ & {$[-0.31,+0.09]$} \\
$d_{Y Y}$ & $0.11 \pm 0.10 \pm 0.02$ & {$[-0.09,+0.31]$} \\
$d_{X Y}$ & $-0.04 \pm 0.10 \pm 0.01$ & {$[-0.24,+0.16]$} \\
$d_{X Z}$ & $0.14 \pm 0.07 \pm 0.02$ & {$[-0.01,+0.29]$} \\
$d_{Y Z}$ & $-0.02 \pm 0.07 \pm<0.01$ & {$[-0.16,+0.13]$} \\
\hline \hline
\end{tabular}

orientation of the detector, also contribute to a potential further increase in sensitivity to Lorentz violating effects in the $t \bar{t}$ event rate at ATLAS. We conservatively estimate that ATLAS may be able to improve on the limits presented here by almost an order of magnitude, and may be sensitive enough to set the first limits on the linear combination $c_{\mu \nu}$.

We have found no evidence that the top quark violates Lorentz symmetry. We have presented the first search for Lorentz violation in the top quark sector and 
have found the corresponding coefficients within the Standard-Model Extension to be consistent with zero. It is important to test all particle species, as different particles can have distinct Lorentz-violating properties. This analysis represents the first such constraints on Lorentz violation in the top quark sector. 


\section{Bibliography}

[1] V.M. Abazov et al. (D0 Collaboration), in press [arXiv:1203.6106].

[2] D. Colladay and V.A. Kostelecký, Phys. Rev. D 58, 116002 (1998); V.A. Kostelecký, Phys. Rev. D 69, 105009 (2004).

[3] F. Englert and R. Brout, Phys. Rev. Lett. 13, 321 (1964); P.W. Higgs, Phys. Rev. Lett. 13, 508 (1964); G.S. Guralnik, C.R. Hagen, and T.W. Kibble, Phys. Rev. Lett. 13, 321 (1964).

[4] ATLAS Collaboration, ATLAS-CONF-2012-093 (2012); CMS Collaboration, CMS-PAS-HIG-12-020 (2012).

[5] P.A.M. Dirac, Proc. R. Soc. Lond. A 117, 610 (1928).

[6] C.D. Anderson, Phys. Rev. 43491 (1933).

[7] C. Amole et al. (ALPHA Collaboration), Nature 483439 (2012).

[8] S.W. Herb et al., Phys. Rev. Lett. 39252 (1977).

[9] G. Arnison et al. (UA1 Collaboration), Phys. Lett. B 122103 (1983); M. Banner et al. (UA2 Collaboration), Phys. Lett. B 122476 (1983); G. Arnison et al. 
(UA1 Collaboration), Phys. Lett. B 129273 (1983); G. Arnison et al. (UA1 Collaboration),

[10] Phys. Lett. B 126398 (1983); P. Bagnaia et al. (UA2 Collaboration), Phys. Lett. B 129130 (1983).

[11] F. Abe et al. (CDF Collaboration), Phys. Rev. Lett. 742626 (1995); S. Abachi et al. (DØ Collaboration), Phys. Rev. Lett. 742632 (1995).

[12] A. Einstein, Annalen der Physik 17, 891 (1905).

[13] O.W. Greenberg, Phys. Rev. Lett. 89, 231602 (2002).

[14] F. Halzen and A.D. Martin, Quarks 83 Leptons (John Wiley \& Sons, New York, 1984).

[15] V.A. Kostelecký and N. Russell, Rev. Mod. Phys. 83, 11 (2011).

[16] D.L. Anderson, M. Sher, and I. Turan, Phys. Rev. D 70, 016001 (2004).

[17] P. Adamson et al. (MINOS Collaboration), Phys. Rev. Lett. 101, 151601 (2008).

[18] P. Adamson et al. (MINOS Collaboration), Phys. Rev. Lett. 105, 151601 (2010).

[19] R. Abbasi et al. (IceCube Collaboration), Phys. Rev. D 82, 112003 (2010).

[20] B. Aubert et al. (BaBar Collaboration), Phys. Rev. Lett. 100, 131802 (2008).

[21] V.A. Kostelecký and R.J. Van Kooten, Phys. Rev. D 82, 101702(R) (2010).

[22] M.S. Berger and V.A. Kostelecký, Notes on Lorentz and CPT Violation in TopQuark Production and Decay, IUHET 530. 
[23] T. Sjöstrand, S. Mrenna and P. Skands, J. High Energy Phys. 05, 26 (2006).

[24] H.T. Edwards, Ann. Rev. Nucl. Part. Sci. 35, 605 (1985); S.D. Holmes (ed.), Tevatron Run II Handbook (1998) FERMILAB-TM-2484-1998; S.D. Holmes, R. Moore, and V. Shiltsev, JINST 6, T08001 (2011).

[25] S. Abachi et al. (DØ Collaboration), Nucl. Instrum. Methods Phys. Res. A 338, 185 (1994); V.M. Abazov et al. (DØ Collaboration), Nucl. Instrum. Methods Phys. Res. A 565, 463 (2006).

[26] R. Kehoe, M. Narain, and A. Kumar, Int. J. Mod. Phys. A 23, 353 (2008).

[27] V.M. Abazov et al. (DØ Collaboration), Phys. Rev. D 84, 032004 (2011); V.M. Abazov et al. (DØ Collaboration), Phys. Rev. D 84, 112005 (2011); V.M. Abazov et al. (DØ Collaboration), Phys. Rev. D 85, 091104(R) (2012).

[28] V.M. Abazov et al. (DØ Collaboration), Phys. Rev. Lett. 108, 151804 (2012).

[29] V.M. Abazov et al. (DØ Collaboration), Phys. Rev. Lett. 107, 241803 (2011). V.M. Abazov et al. (DØ Collaboration), Phys. Rev. D 85, 052001 (2012).

[30] V.M. Abazov et al. (DØ Collaboration), Phys. Rev. Lett. 101, 241801 (2008).

[31] V.M. Abazov et al. (DØ Collaboration), Phys. Lett. B 659, 856 (2008); V.M. Abazov et al. (DØ Collaboration), Phys. Rev. Lett. 100, 241803 (2008).

[32] V.M. Abazov et al. (DØ Collaboration), Phys. Lett. B 681, 224 (2009); V.M. Abazov et al. (DØ Collaboration), Phys. Lett. B 693, 95 (2010).

[33] V.M. Abazov et al. (DØ Collaboration), Phys. Rev. Lett. 108, 131802 (2012). 
[34] T. Aaltonen et al. (CDF and DØ Collaborations), Phys. Rev. Lett. 104, 061802 (2010).

[35] B. Casey et al., DØ Note 5945 (2009).

[36] V.M. Abazov et al. (DØ Collaboration), Phys. Rev. D 84, 012008 (2011).

[37] V.M. Abazov et al. (DØ Collaboration), Phys. Rev. Lett. 100, 192004 (2008).

[38] O. Brandt et al., DØ Note 6028 (2010).

[39] G.C. Blazey et al., in Proceedings of the Workshop: QCD and Weak Boson Physics in Run II, edited by U. Baur, R.K. Ellis, and D. Zeppenfeld (Fermilab, Batavia, IL, 2000) p. 47.

[40] V.M. Abazov et al. (DØ Collaboration), Nucl. Instrum. Methods Phys. Res. A 620, 490 (2010).

[41] ATLAS Collaboration, ATLAS-CONF-2012-024 (2012).

[42] ATLAS Collaboration, JINST 3, S08003 (2008).

[43] L. Evans and P. Bryant, JINST 3, 208001 (2008).

[44] ATLAS Collaboration, ATLAS-CONF-2011-121 (2011); ATLAS Collaboration, Phys. Lett. B 711, 244 (2012); ATLAS Collaboration, JHEP 1205, 059 (2012); ATLAS Collaboration, in press [arXiv:1205.2067].

[45] ATLAS Collaboration, in press [arXiv:1203.5755].

[46] ATLAS Collaboration, in press [arXiv:1205.5371]. 
[47] N.R. Lomb, Astrophys. Space Sci. 39, 447 (1976); J.D. Scargle, Astrophys. J. 263, 835 (1982).

[48] ATLAS Collaboration, in press [arXiv:1205.3130]. 


\section{Appendix A}

\section{List of Triggers for DØ Data}

We reproduce the trigger lists used by the $t \bar{t}$ cross section analysis performed at $\mathrm{D} \varnothing$ using the lepton+jets channel in proton-antiproton collisions at $\sqrt{s}=1.96 \mathrm{TeV}$ [38].

Table A.1: Trigger and corresponding integrated luminosity analyzed for the $e+$ jets sample in Run IIa.

\begin{tabular}{lcc}
\hline \hline Triggerlist Version & Trigger & Integrated luminosity $\left[\mathrm{pb}^{-1}\right]$ \\
\hline V8.0-V14.99 & JT_125TT & 1078.81 \\
\hline \hline
\end{tabular}


Table A.2: Trigger and corresponding integrated luminosity analyzed for the $\mu+$ jets sample in Run IIa.

\begin{tabular}{lcc}
\hline \hline Triggerlist Version & Trigger & Integrated luminosity $\left[\mathrm{pb}^{-1}\right]$ \\
\hline V8.0-V9.0 & MU_JT20_L2M0 & 24.80 \\
V9.0-V10.0 & MU_JT20_L2M0 & 25.01 \\
V10.0-V11.0 & MU_JT20_L2M0 & 10.70 \\
V11.0-V12.0 & MU_JT20_L2M0 & 65.85 \\
V12.0-V13.0 & MU_JT25_L2M0 & 231.84 \\
V13.0-V13.2 & MUJ2_JT25 & 31.86 \\
V13.2-V13.3 & MUJ2_JT25_LM3 & 16.11 \\
V13.3-V14.0 & MUJ2_JT30_LM3 & 255.80 \\
V14.0-V14.2 & MUJ1_JT25_LM3 & 0.01 \\
V14.2-V14.3 & MUJ1_JT25_ILM3 & 21.89 \\
V14.3-V15.0 & MUJ1_JT35_LM3 & 317.17 \\
\hline \hline
\end{tabular}

Table A.3: Trigger and corresponding integrated luminosity analyzed for the $e+$ jets and $\mu+$ jets samples in Run IIb.

\begin{tabular}{lcc}
\hline \hline Triggerlist Version & Trigger & Integrated luminosity $\left[\mathrm{pb}^{-1}\right]$ \\
\hline V15.0-V15.99 & JT125_L3J125 & 1619.77 \\
V16.0-V16.99 & JT125_L3J125 & 2661.89 \\
\hline \hline
\end{tabular}


Appendix B

Contributions to $A_{P}^{\alpha \beta}$ and $A_{F}^{\alpha \beta}$ 

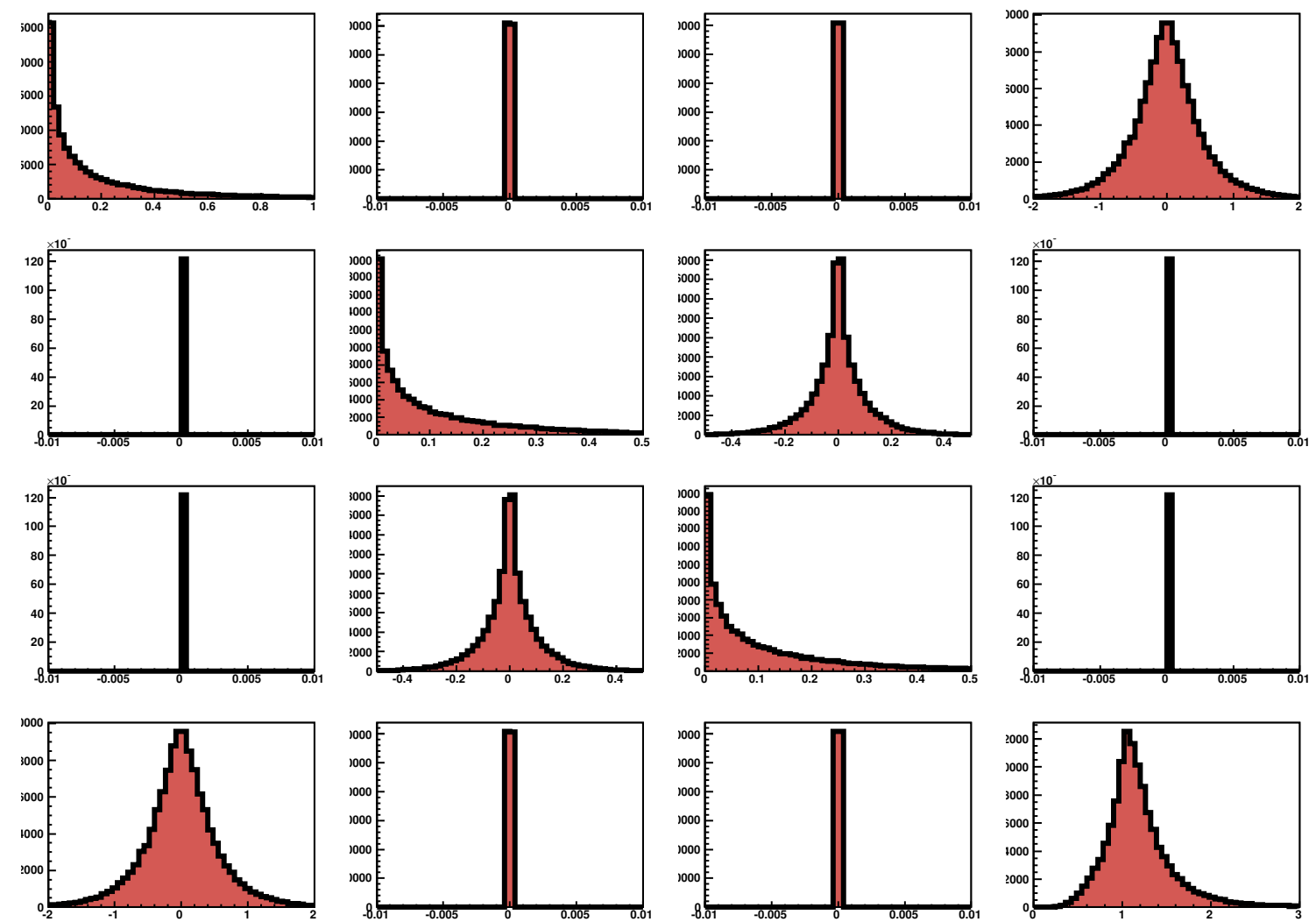

Figure B.1: Distribution of event-by-event contributions to the elements of $A_{P}^{\alpha \beta}$ in the simulated $e+$ jets sample. Rows and columns are ordered $(t, x, y, z)$. 

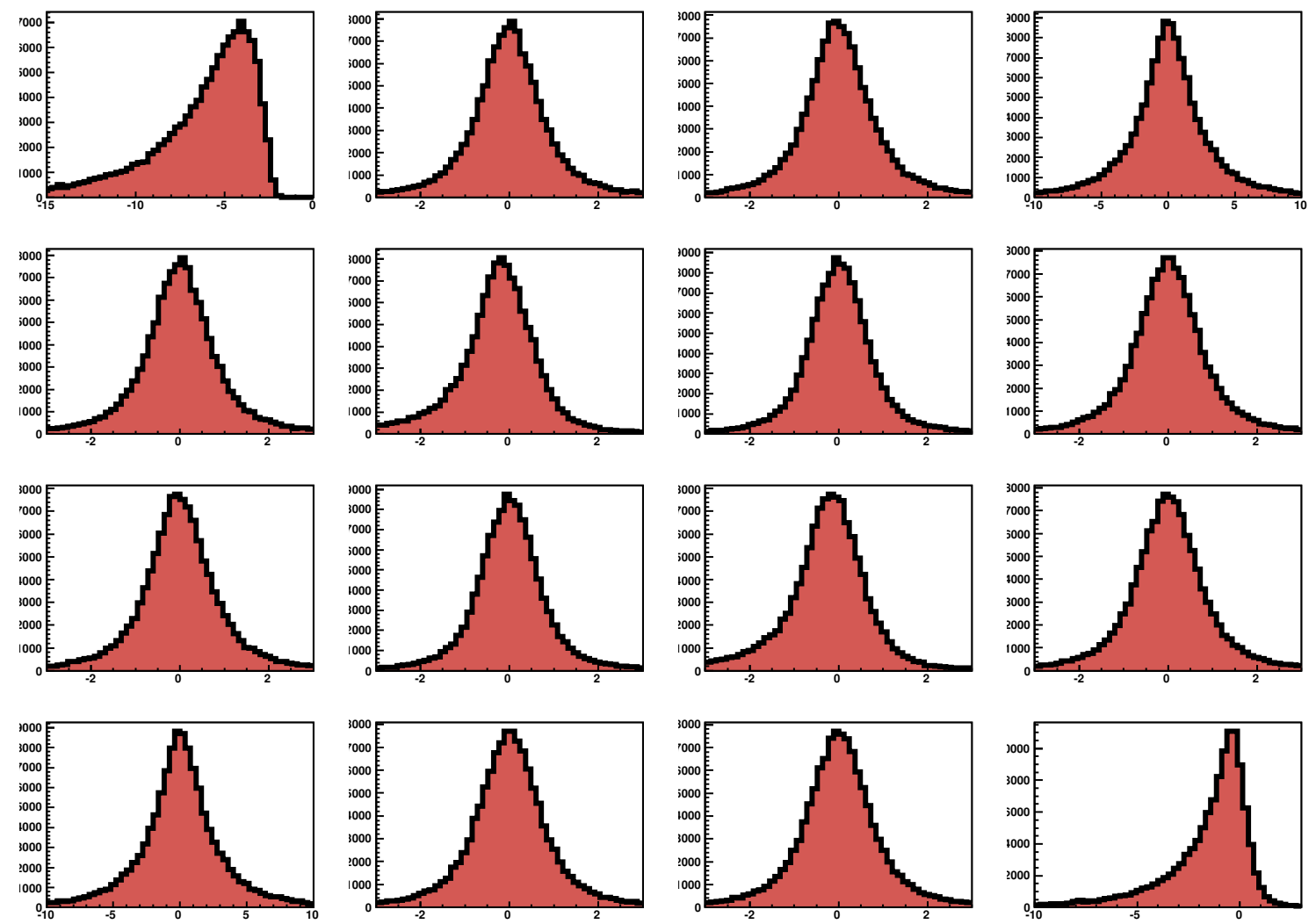

Figure B.2: Distribution of event-by-event contributions to the elements of $A_{F}^{\alpha \beta}$ in the simulated $e+$ jets sample. Rows and columns are ordered $(t, x, y, z)$. 

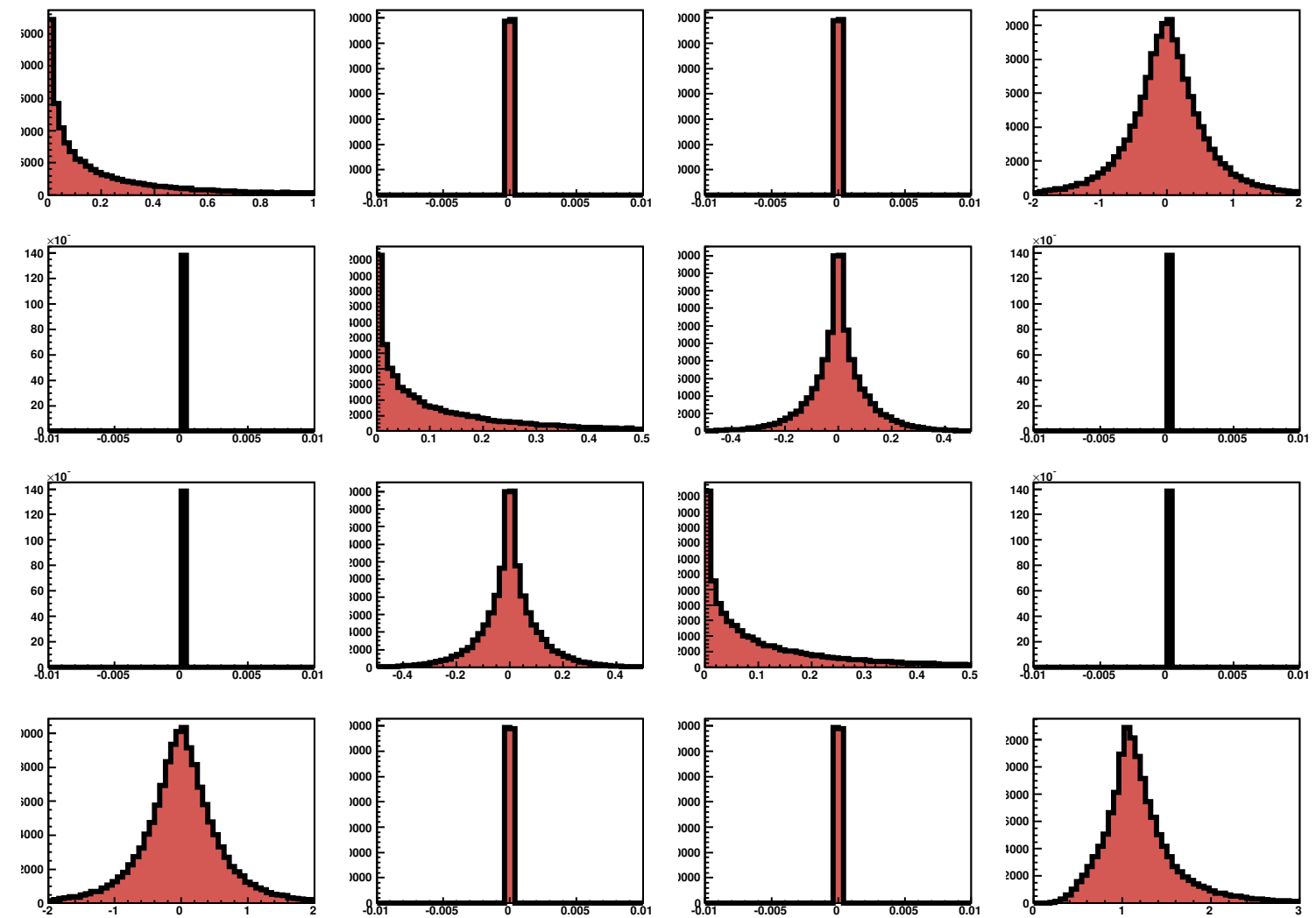

Figure B.3: Distribution of event-by-event contributions to the elements of $A_{P}^{\alpha \beta}$ in the simulated $\mu+$ jets sample. Rows and columns are ordered $(t, x, y, z)$. 

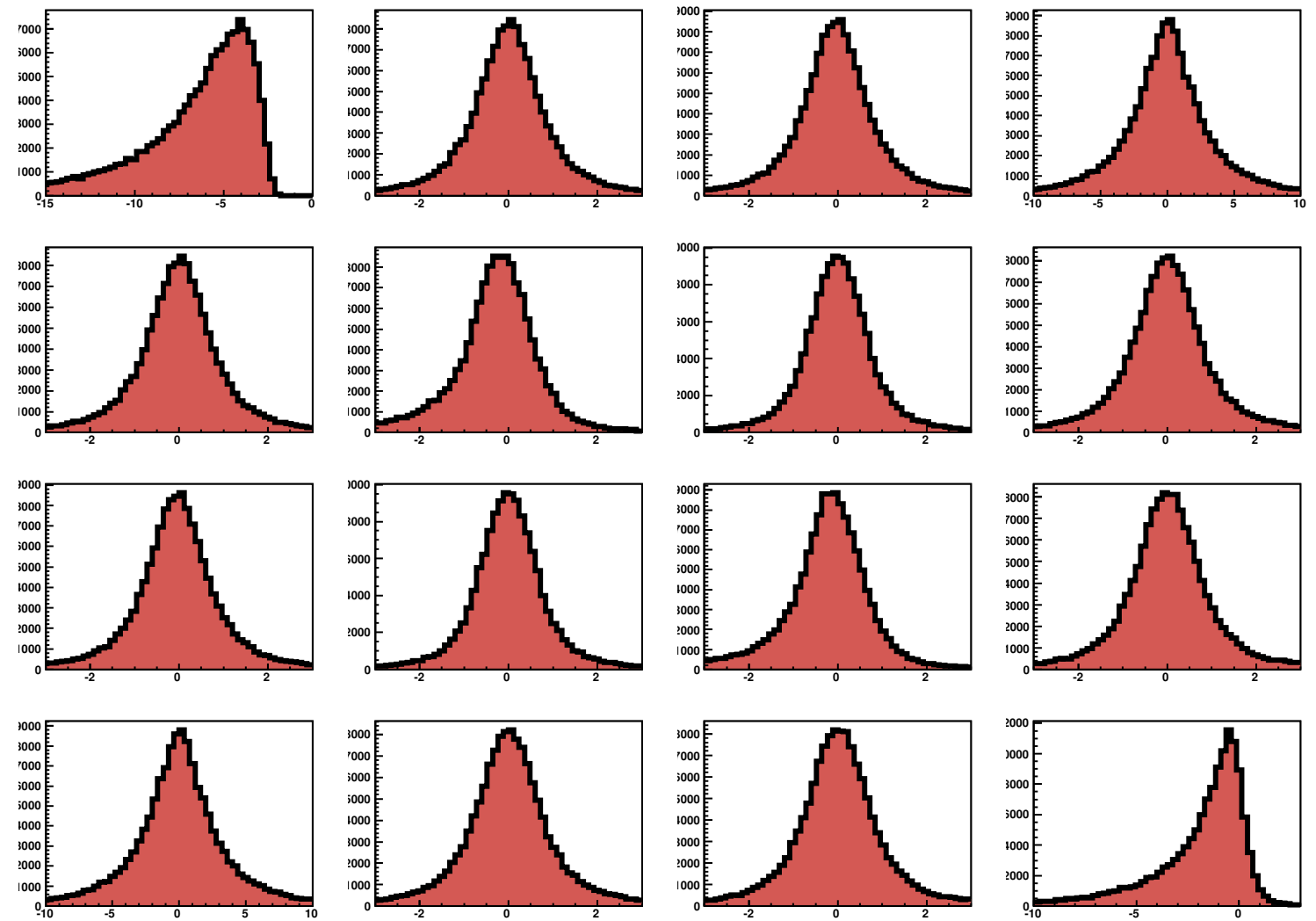

Figure B.4: Distribution of event-by-event contributions to the elements of $A_{F}^{\alpha \beta}$ in the simulated $\mu+$ jets sample. Rows and columns are ordered $(t, x, y, z)$. 


\section{Appendix $\mathrm{C}$}

\section{Systematics in MC Summation}

The event selection used for the Monte Carlo samples is changed by increasing and decreasing three parameters that most affect $t \bar{t}$ reconstruction by one standard deviation: jet energy scale (JES), jet energy resolution (JER), and jet identification (JETID). The $A_{P}^{\alpha \beta}$ and $A_{F}^{\alpha \beta}$ matrices are recalculated after separate changes for each of these sources. Tables C.1-C.16 summarize the systematic uncertainties for the diagonal elements that contribute to Eq. (3.25) for events collected with the DØ detector. 


\section{C.1 Uncertainties for $e+$ jets}

Table C.1: Uncertainties on $A_{P}^{t t}$

\begin{tabular}{crr}
\hline \hline Uncertainty & $-1 \mathrm{sd}$ & $+1 \mathrm{sd}$ \\
\hline JER & 0.0002 & -0.0002 \\
JES & -0.0006 & 0.0003 \\
JETID & -0.0002 & -0.0003 \\
STAT & -0.0010 & 0.0010 \\
\hline Total & 0.0012 & 0.0011 \\
\hline \hline
\end{tabular}

Table C.3: Uncertainties on $A_{P}^{x x}$

\begin{tabular}{crr}
\hline \hline Uncertainty & $-1 \mathrm{sd}$ & $+1 \mathrm{sd}$ \\
\hline JER & 0.0004 & -0.0003 \\
JES & 0.0009 & -0.0009 \\
JETID & 0.0001 & 0.0001 \\
STAT & -0.0004 & 0.0004 \\
\hline Total & 0.0011 & 0.0010 \\
\hline \hline
\end{tabular}

Table C.5: Uncertainties on $A_{P}^{y y}$

\begin{tabular}{crr}
\hline \hline Uncertainty & $-1 \mathrm{sd}$ & $+1 \mathrm{sd}$ \\
\hline JER & -0.0001 & -0.0002 \\
JES & 0.0011 & -0.0011 \\
JETID & 0.0000 & 0.0000 \\
STAT & -0.0004 & 0.0004 \\
\hline Total & 0.0012 & 0.0012 \\
\hline \hline
\end{tabular}

Table C.2: Uncertainties on $A_{F}^{t t}$

\begin{tabular}{crr}
\hline \hline Uncertainty & $-1 \mathrm{sd}$ & $+1 \mathrm{sd}$ \\
\hline JER & 0.0011 & -0.0436 \\
JES & 0.0017 & 0.0112 \\
JETID & -0.0063 & -0.0062 \\
STAT & -0.0920 & 0.0920 \\
\hline Total & 0.0922 & 0.1026 \\
\hline \hline
\end{tabular}

Table C.4: Uncertainties on $A_{F}^{x x}$

\begin{tabular}{crr}
\hline \hline Uncertainty & $-1 \mathrm{sd}$ & $+1 \mathrm{sd}$ \\
\hline JER & -0.0114 & 0.0044 \\
JES & -0.0132 & 0.0097 \\
JETID & 0.0001 & 0.0000 \\
STAT & -0.0133 & 0.0133 \\
\hline Total & 0.0219 & 0.0170 \\
\hline \hline
\end{tabular}

Table C.6: Uncertainties on $A_{F}^{y y}$

\begin{tabular}{crr}
\hline \hline Uncertainty & $-1 \mathrm{sd}$ & $+1 \mathrm{sd}$ \\
\hline JER & -0.0097 & 0.0069 \\
JES & -0.0122 & 0.0111 \\
JETID & -0.0001 & -0.0001 \\
STAT & -0.0147 & 0.0147 \\
\hline Total & 0.0214 & 0.0197 \\
\hline \hline
\end{tabular}


Table C.7: Uncertainties on $A_{P}^{z z}$

\begin{tabular}{crr}
\hline \hline Uncertainty & $-1 \mathrm{sd}$ & $+1 \mathrm{sd}$ \\
\hline JER & 0.0000 & -0.0003 \\
JES & -0.0002 & -0.0004 \\
JETID & -0.0002 & 0.0002 \\
STAT & -0.0011 & 0.0011 \\
\hline Total & 0.0011 & 0.0012 \\
\hline \hline
\end{tabular}

Table C.8: Uncertainties on $A_{F}^{z z}$

\begin{tabular}{crr}
\hline \hline Uncertainty & $-1 \mathrm{sd}$ & $+1 \mathrm{sd}$ \\
\hline JER & -0.0204 & 0.0025 \\
JES & -0.0022 & 0.0083 \\
JETID & -0.0005 & -0.0004 \\
STAT & -0.0484 & 0.0484 \\
\hline Total & 0.0526 & 0.0492 \\
\hline \hline
\end{tabular}

\section{C.2 Uncertainties for $\mu+$ jets}

Table C.9: Uncertainties on $A_{P}^{t t}$

\begin{tabular}{crr}
\hline \hline Uncertainty & $-1 \mathrm{sd}$ & $+1 \mathrm{sd}$ \\
\hline JER & -0.0005 & 0.0001 \\
JES & -0.0004 & 0.0004 \\
JETID & -0.0001 & -0.0001 \\
STAT & -0.0010 & 0.0010 \\
\hline Total & 0.0012 & 0.0011 \\
\hline \hline
\end{tabular}

Table C.11: Uncertainties on $A_{P}^{x x}$

\begin{tabular}{crr}
\hline \hline Uncertainty & $-1 \mathrm{sd}$ & $+1 \mathrm{sd}$ \\
\hline JER & 0.0001 & 0.0003 \\
JES & 0.0009 & -0.0008 \\
JETID & 0.0001 & 0.0001 \\
STAT & -0.0004 & 0.0004 \\
\hline Total & 0.0010 & 0.0009 \\
\hline \hline
\end{tabular}

Table C.10: Uncertainties on $A_{F}^{t t}$

\begin{tabular}{crr}
\hline \hline Uncertainty & $-1 \mathrm{sd}$ & $+1 \mathrm{sd}$ \\
\hline JER & 0.0627 & -0.0191 \\
JES & 0.0382 & -0.0255 \\
JETID & -0.0068 & -0.0068 \\
STAT & -0.0684 & 0.0684 \\
\hline Total & 0.1006 & 0.0758 \\
\hline \hline
\end{tabular}

Table C.12: Uncertainties on $A_{F}^{x x}$

\begin{tabular}{crr}
\hline \hline Uncertainty & $-1 \mathrm{sd}$ & $+1 \mathrm{sd}$ \\
\hline JER & -0.0018 & 0.0073 \\
JES & -0.0117 & 0.0161 \\
JETID & -0.0008 & -0.0007 \\
STAT & -0.0107 & 0.0107 \\
Total & 0.0160 & 0.0207 \\
\hline \hline
\end{tabular}


Table C.13: Uncertainties on $A_{P}^{y y}$

\begin{tabular}{crr}
\hline \hline Uncertainty & $-1 \mathrm{sd}$ & $+1 \mathrm{sd}$ \\
\hline JER & -0.0001 & 0.0001 \\
JES & 0.0007 & -0.0008 \\
JETID & 0.0000 & 0.0000 \\
STAT & -0.0004 & 0.0004 \\
\hline Total & 0.0008 & 0.0009 \\
\hline \hline
\end{tabular}

Table C.15: Uncertainties on $A_{P}^{z z}$

\begin{tabular}{crr}
\hline \hline Uncertainty & $-1 \mathrm{sd}$ & $+1 \mathrm{sd}$ \\
\hline JER & -0.0009 & 0.0005 \\
JES & 0.0001 & 0.0001 \\
JETID & -0.0001 & -0.0001 \\
STAT & -0.0012 & 0.0012 \\
\hline Total & 0.0015 & 0.0013 \\
\hline \hline
\end{tabular}

Table C.14: Uncertainties on $A_{F}^{y y}$

\begin{tabular}{crr}
\hline \hline Uncertainty & $-1 \mathrm{sd}$ & $+1 \mathrm{sd}$ \\
\hline JER & -0.0040 & 0.0055 \\
JES & -0.0109 & 0.0135 \\
JETID & 0.0004 & 0.0004 \\
STAT & -0.0097 & 0.0097 \\
\hline Total & 0.0151 & 0.0175 \\
\hline \hline
\end{tabular}

Table C.16: Uncertainties on $A_{F}^{z z}$

\begin{tabular}{crr}
\hline \hline Uncertainty & $-1 \mathrm{sd}$ & $+1 \mathrm{sd}$ \\
\hline JER & 0.0043 & 0.0094 \\
JES & 0.0107 & -0.0087 \\
JETID & -0.0009 & -0.0008 \\
STAT & -0.0274 & 0.0274 \\
\hline Total & 0.0297 & 0.0303 \\
\hline \hline
\end{tabular}




\section{Appendix D}

\section{$\ell+n$-jets Cross-Checks}

\section{D.1 $\ell+2$-jets cross-check}

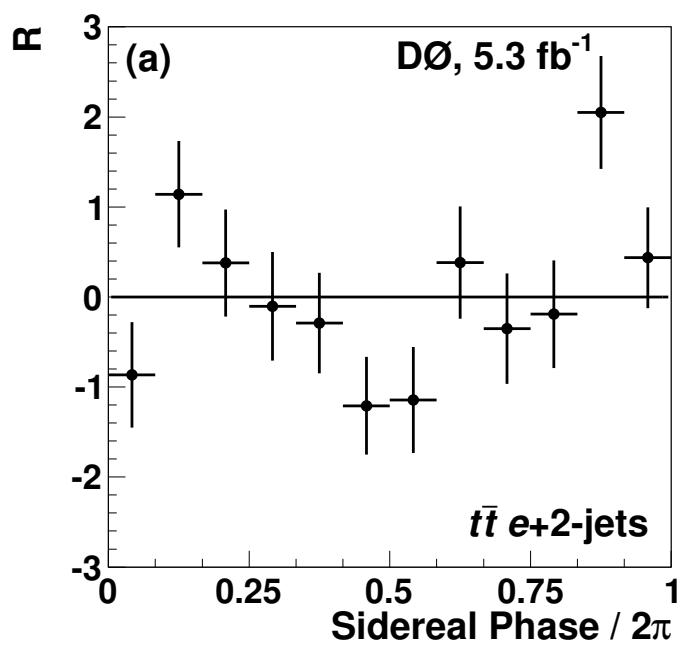

(a) $e+2$-jets $t \bar{t}$ candidates.

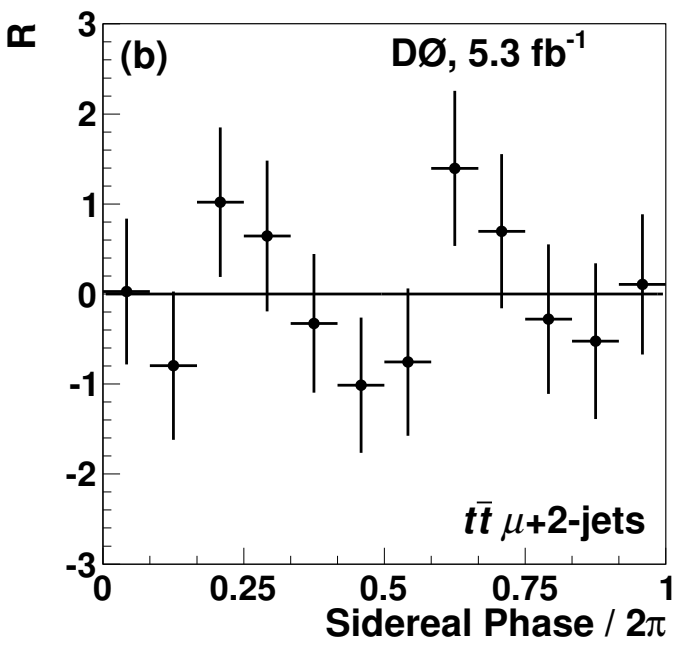

(b) $\mu+2$-jets $t \bar{t}$ candidates.

Figure D.1: Number of $\ell+2$-jets $t \bar{t}$ candidates per two sidereal hours. 


\section{D.1.1 Electron amplitudes}

Table D.1: Amplitude extracted from $e+2$-jets $t \bar{t}$ candidates, with phase fixed appropriate to each coefficient case and assuming $\left(c_{R}\right)_{\mu \nu}=0$.

\begin{tabular}{ccc}
\hline \hline Coefficient Case & Amplitude \pm Uncertainty & Significance $(\mathrm{sd})$ \\
\hline$X X$ & $-0.0184 \pm 0.0326$ & 0.565 \\
$X Y$ & $-0.0513 \pm 0.0329$ & 1.56 \\
$X Z$ & $-0.0736 \pm 0.0314$ & 2.35 \\
$Y Z$ & $-0.0609 \pm 0.0320$ & 1.91 \\
\hline
\end{tabular}

Table D.2: Amplitude extracted from $e+2$-jets $t \bar{t}$ candidates, with phase fixed appropriate to each coefficient case and assuming $\left(c_{L}\right)_{\mu \nu}=0$.

\begin{tabular}{ccc}
\hline \hline Coefficient Case & Amplitude \pm Uncertainty & Significance (sd) \\
\hline$X X$ & $0.0184 \pm 0.0326$ & 0.565 \\
$X Y$ & $0.0513 \pm 0.0329$ & 1.56 \\
$X Z$ & $0.0653 \pm 0.0315$ & 2.07 \\
$Y Z$ & $0.0703 \pm 0.0318$ & 2.21 \\
\hline \hline
\end{tabular}

Table D.3: Amplitude extracted from $e+2$-jets $t \bar{t}$ candidates, with phase fixed appropriate to each coefficient case and assuming $d_{\mu \nu}=0$.

\begin{tabular}{ccc}
\hline \hline Coefficient Case & Amplitude \pm Uncertainty & Significance (sd) \\
\hline$X X$ & $0.0184 \pm 0.0326$ & 0.565 \\
$X Y$ & $0.0513 \pm 0.0329$ & 1.56 \\
$X Z$ & $0.0343 \pm 0.0320$ & 1.07 \\
$Y Z$ & $0.0879 \pm 0.0313$ & 2.81 \\
\hline \hline
\end{tabular}

Table D.4: Amplitude extracted from $e+2$-jets $t \bar{t}$ candidates, with phase fixed appropriate to each coefficient case and assuming $c_{\mu \nu}=0$.

\begin{tabular}{ccc}
\hline \hline Coefficient Case & Amplitude \pm Uncertainty & Significance (sd) \\
\hline$X X$ & $-0.0184 \pm 0.0326$ & 0.565 \\
$X Y$ & $-0.0513 \pm 0.0329$ & 1.56 \\
$X Z$ & $-0.0690 \pm 0.0314$ & 2.2 \\
$Y Z$ & $-0.0665 \pm 0.0319$ & 2.09 \\
\hline \hline
\end{tabular}




\section{D.1.2 Muon amplitudes}

Table D.5: Amplitude extracted from $\mu+2$-jets $t \bar{t}$ candidates, with phase fixed appropriate to each coefficient case and assuming $\left(c_{R}\right)_{\mu \nu}=0$.

\begin{tabular}{ccc}
\hline \hline Coefficient Case & Amplitude \pm Uncertainty & Significance $(\mathrm{sd})$ \\
\hline$X X$ & $-0.0663 \pm 0.0386$ & 1.72 \\
$X Y$ & $-0.0352 \pm 0.0389$ & 0.905 \\
$X Z$ & $-0.0088 \pm 0.0371$ & 0.237 \\
$Y Z$ & $-0.0012 \pm 0.0378$ & 0.0306 \\
\hline
\end{tabular}

Table D.6: Amplitude extracted from $\mu+2$-jets $t \bar{t}$ candidates, with phase fixed appropriate to each coefficient case and assuming $\left(c_{L}\right)_{\mu \nu}=0$.

\begin{tabular}{ccc}
\hline \hline Coefficient Case & Amplitude \pm Uncertainty & Significance (sd) \\
\hline$X X$ & $0.0663 \pm 0.0386$ & 1.72 \\
$X Y$ & $0.0352 \pm 0.0389$ & 0.905 \\
$X Z$ & $0.0087 \pm 0.0372$ & 0.233 \\
$Y Z$ & $0.0022 \pm 0.0376$ & 0.0594 \\
\hline \hline
\end{tabular}

Table D.7: Amplitude extracted from $\mu+2$-jets $t \bar{t}$ candidates, with phase fixed appropriate to each coefficient case and assuming $d_{\mu \nu}=0$.

\begin{tabular}{ccc}
\hline \hline Coefficient Case & Amplitude \pm Uncertainty & Significance (sd) \\
\hline$X X$ & $0.0663 \pm 0.0386$ & 1.72 \\
$X Y$ & $0.0352 \pm 0.0389$ & 0.905 \\
$X Z$ & $-0.0034 \pm 0.0374$ & 0.092 \\
$Y Z$ & $0.0084 \pm 0.0375$ & 0.223 \\
\hline \hline
\end{tabular}

Table D.8: Amplitude extracted from $\mu+2$-jets $t \bar{t}$ candidates, with phase fixed appropriate to each coefficient case and assuming $c_{\mu \nu}=0$.

\begin{tabular}{ccc}
\hline \hline Coefficient Case & Amplitude \pm Uncertainty & Significance (sd) \\
\hline$X X$ & $-0.0663 \pm 0.0386$ & 1.72 \\
$X Y$ & $-0.0352 \pm 0.0389$ & 0.905 \\
$X Z$ & $-0.0088 \pm 0.0371$ & 0.235 \\
$Y Z$ & $-0.0017 \pm 0.0377$ & 0.0449 \\
\hline
\end{tabular}




\section{D.2 $\ell+3$-jets cross-check}

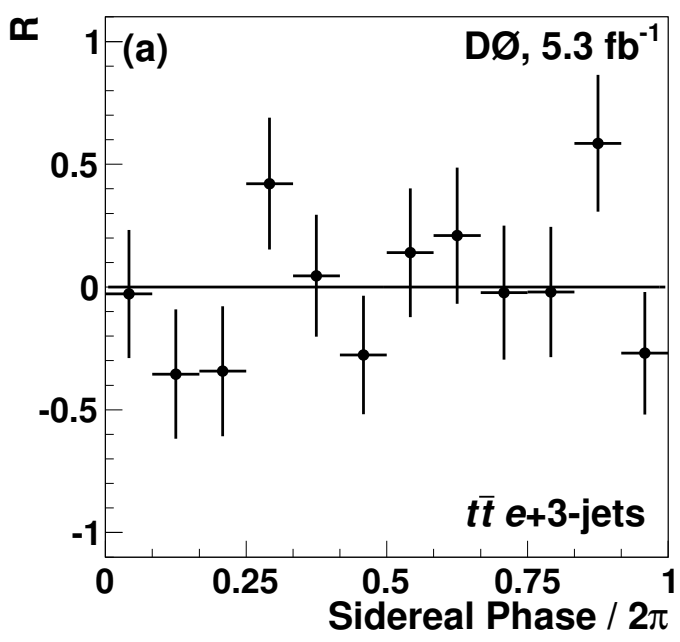

(a) $e+3$-jets $t \bar{t}$ candidates.

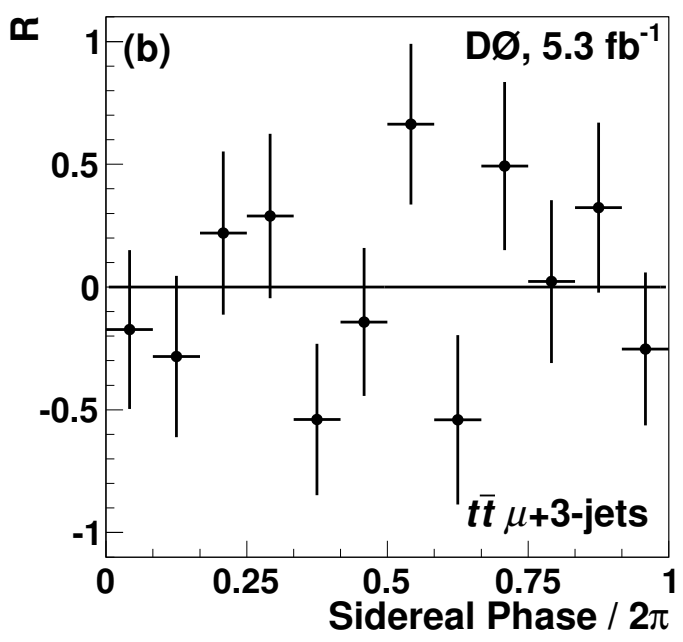

(b) $\mu+3$-jets $t \bar{t}$ candidates.

Figure D.2: Number of $\ell+3$-jets $t \bar{t}$ candidates per two sidereal hours. 


\section{D.2.1 Electron amplitudes}

Table D.9: Amplitude extracted from $e+3$-jets $t \bar{t}$ candidates, with phase fixed appropriate to each coefficient case and assuming $\left(c_{R}\right)_{\mu \nu}=0$.

\begin{tabular}{ccc}
\hline \hline Coefficient Case & Amplitude \pm Uncertainty & Significance $(\mathrm{sd})$ \\
\hline$X X$ & $0.0325 \pm 0.0507$ & 0.641 \\
$X Y$ & $-0.0611 \pm 0.0511$ & 1.2 \\
$X Z$ & $-0.0167 \pm 0.0487$ & 0.343 \\
$Y Z$ & $0.0567 \pm 0.0497$ & 1.14 \\
\hline
\end{tabular}

Table D.10: Amplitude extracted from $e+3$-jets $t \bar{t}$ candidates, with phase fixed appropriate to each coefficient case and assuming $\left(c_{L}\right)_{\mu \nu}=0$.

\begin{tabular}{ccc}
\hline \hline Coefficient Case & Amplitude \pm Uncertainty & Significance (sd) \\
\hline$X X$ & $-0.0325 \pm 0.0507$ & 0.641 \\
$X Y$ & $0.0611 \pm 0.0511$ & 1.2 \\
$X Z$ & $0.0245 \pm 0.0490$ & 0.501 \\
$Y Z$ & $-0.0531 \pm 0.0494$ & 1.08 \\
\hline \hline
\end{tabular}

Table D.11: Amplitude extracted from $e+3$-jets $t \bar{t}$ candidates, with phase fixed appropriate to each coefficient case and assuming $d_{\mu \nu}=0$.

\begin{tabular}{ccc}
\hline \hline Coefficient Case & Amplitude \pm Uncertainty & Significance (sd) \\
\hline$X X$ & $-0.0325 \pm 0.0507$ & 0.641 \\
$X Y$ & $0.0611 \pm 0.0511$ & 1.2 \\
$X Z$ & $0.0441 \pm 0.0497$ & 0.889 \\
$Y Z$ & $-0.0381 \pm 0.0487$ & 0.782 \\
\hline \hline
\end{tabular}

Table D.12: Amplitude extracted from $e+3$-jets $t \bar{t}$ candidates, with phase fixed appropriate to each coefficient case and assuming $c_{\mu \nu}=0$.

\begin{tabular}{ccc}
\hline \hline Coefficient Case & Amplitude \pm Uncertainty & Significance (sd) \\
\hline$X X$ & $0.0325 \pm 0.0507$ & 0.641 \\
$X Y$ & $-0.0611 \pm 0.0511$ & 1.2 \\
$X Z$ & $-0.0212 \pm 0.0488$ & 0.434 \\
$Y Z$ & $0.0547 \pm 0.0495$ & 1.11 \\
\hline
\end{tabular}




\section{D.2.2 Muon amplitudes}

Table D.13: Amplitude extracted from $\mu+3$-jets $t \bar{t}$ candidates, with phase fixed appropriate to each coefficient case and assuming $\left(c_{R}\right)_{\mu \nu}=0$.

\begin{tabular}{ccc}
\hline \hline Coefficient Case & Amplitude \pm Uncertainty & Significance $(\mathrm{sd})$ \\
\hline$X X$ & $-0.0436 \pm 0.0620$ & 0.704 \\
$X Y$ & $-0.0468 \pm 0.0624$ & 0.751 \\
$X Z$ & $-0.0229 \pm 0.0595$ & 0.385 \\
$Y Z$ & $0.0488 \pm 0.0607$ & 0.803 \\
\hline
\end{tabular}

Table D.14: Amplitude extracted from $\mu+3$-jets $t \bar{t}$ candidates, with phase fixed appropriate to each coefficient case and assuming $\left(c_{L}\right)_{\mu \nu}=0$.

\begin{tabular}{ccc}
\hline \hline Coefficient Case & Amplitude \pm Uncertainty & Significance (sd) \\
\hline$X X$ & $0.0436 \pm 0.0620$ & 0.704 \\
$X Y$ & $0.0468 \pm 0.0624$ & 0.751 \\
$X Z$ & $0.0287 \pm 0.0598$ & 0.48 \\
$Y Z$ & $-0.0450 \pm 0.0604$ & 0.746 \\
\hline \hline
\end{tabular}

Table D.15: Amplitude extracted from $\mu+3$-jets $t \bar{t}$ candidates, with phase fixed appropriate to each coefficient case and assuming $d_{\mu \nu}=0$.

\begin{tabular}{ccc}
\hline \hline Coefficient Case & Amplitude \pm Uncertainty & Significance (sd) \\
\hline$X X$ & $0.0436 \pm 0.0620$ & 0.704 \\
$X Y$ & $0.0468 \pm 0.0624$ & 0.751 \\
$X Z$ & $0.0399 \pm 0.0600$ & 0.664 \\
$Y Z$ & $0.0351 \pm 0.0601$ & 0.584 \\
\hline \hline
\end{tabular}

Table D.16: Amplitude extracted from $\mu+3$-jets $t \bar{t}$ candidates, with phase fixed appropriate to each coefficient case and assuming $c_{\mu \nu}=0$.

\begin{tabular}{ccc}
\hline \hline Coefficient Case & Amplitude \pm Uncertainty & Significance (sd) \\
\hline$X X$ & $-0.0436 \pm 0.0620$ & 0.704 \\
$X Y$ & $-0.0468 \pm 0.0624$ & 0.751 \\
$X Z$ & $-0.0258 \pm 0.0596$ & 0.433 \\
$Y Z$ & $0.0470 \pm 0.0605$ & 0.776 \\
\hline
\end{tabular}




\section{D.3 Agreement across samples}

Table D.17: The values of $\chi^{2}$ and probability reflecting consistency with no time dependence for the 4 independent measurements of amplitudes of sidereal oscillations in the cross-check channels $(\ell+n$-jets, where $\ell=e, \mu$ and $n=2,3)$ for each of the 16 phases assumed in this analysis.

\begin{tabular}{llll}
\hline \hline Phase Assumption & $\chi^{2}(4 \mathrm{dof})$ & $\operatorname{Prob}\left(\chi^{2}\right)$ \\
\hline$\left(c_{R}\right)_{\mu \nu}=0$ & $X X$ & 4.167 & 0.384 \\
& $X Y$ & 5.246 & 0.263 \\
& $X Z$ & 5.821 & 0.213 \\
& $Y Z$ & 5.576 & 0.233 \\
\hline$\left(c_{L}\right)_{\mu \nu}=0$ & $X X$ & 4.167 & 0.384 \\
& $X Y$ & 5.246 & 0.263 \\
& $X Z$ & 4.837 & 0.304 \\
& $Y Z$ & 6.616 & 0.158 \\
\hline$d_{\mu \nu}=0$ & $X X$ & 4.167 & 0.384 \\
& $X Y$ & 5.246 & 0.263 \\
& $X Z$ & 2.389 & 0.665 \\
& $Y Z$ & 8.911 & 0.063 \\
\hline$c_{\mu \nu}=0$ & $X X$ & 4.167 & 0.384 \\
& $X Y$ & 5.246 & 0.263 \\
& $X Z$ & 5.271 & 0.261 \\
& $Y Z$ & 6.178 & 0.186 \\
\hline \hline \multicolumn{6}{c}{}
\end{tabular}


Appendix E

\section{Fit Results: Run IIa and Run IIb}




\section{E.1 $\ell+2$-jets in Run IIa}

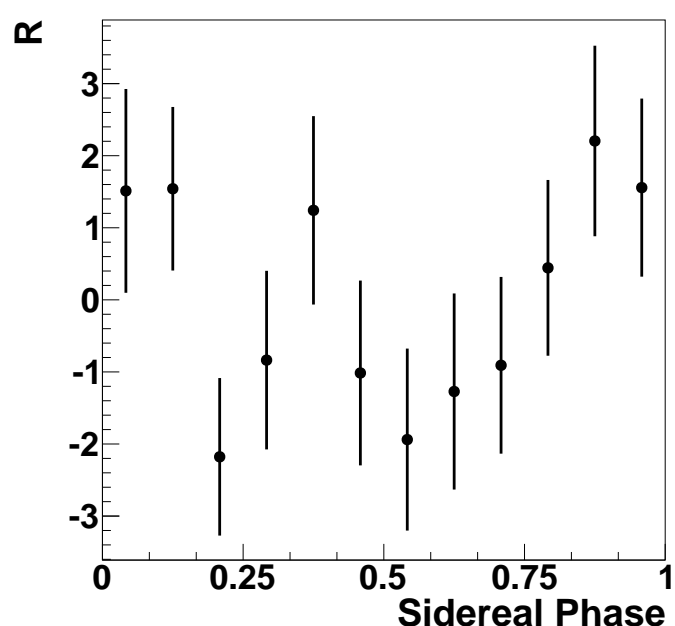

(a) $e+2$-jets $t \bar{t}$ candidates.

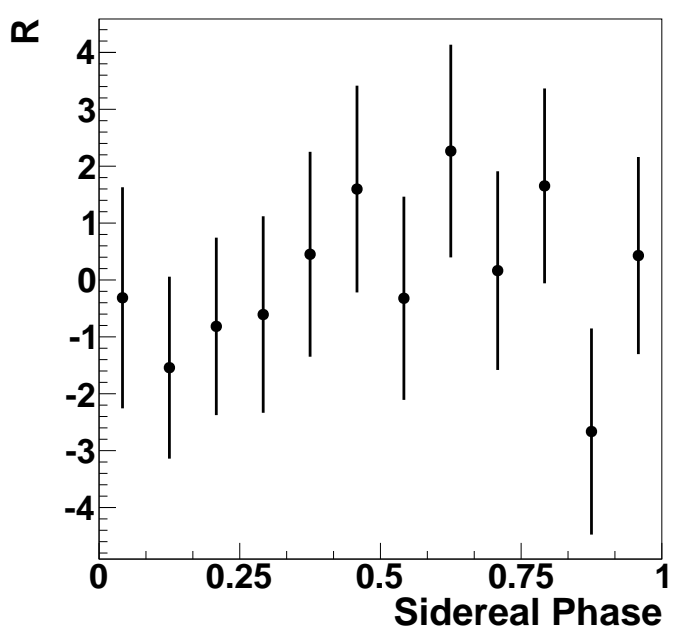

(b) $\mu+2$-jets $t \bar{t}$ candidates.

Figure E.1: Number of $\ell+2$-jets $t \bar{t}$ candidates per two sidereal hours, Run IIa. 


\section{E.1.1 Electron amplitudes}

Table E.1: Amplitude extracted from $e+2$-jets $t \bar{t}$ candidates, with phase fixed appropriate to each coefficient case and assuming $\left(c_{R}\right)_{\mu \nu}=0$.

\begin{tabular}{ccc}
\hline \hline Coefficient Case & Amplitude \pm Uncertainty & Significance (sd) \\
\hline$X X$ & $0.1288 \pm 0.0687$ & 1.88 \\
$X Y$ & $0.0182 \pm 0.0701$ & 0.26 \\
$X Z$ & $-0.1467 \pm 0.0678$ & 2.16 \\
$Y Z$ & $-0.1022 \pm 0.0664$ & 1.54 \\
\hline
\end{tabular}

Table E.2: Amplitude extracted from $e+2$-jets $t \bar{t}$ candidates, with phase fixed appropriate to each coefficient case and assuming $\left(c_{L}\right)_{\mu \nu}=0$.

\begin{tabular}{ccc}
\hline \hline Coefficient Case & Amplitude \pm Uncertainty & Significance (sd) \\
\hline$X X$ & $-0.1288 \pm 0.0687$ & 1.88 \\
$X Y$ & $-0.0182 \pm 0.0701$ & 0.26 \\
$X Z$ & $0.1275 \pm 0.0673$ & 1.9 \\
$Y Z$ & $0.1235 \pm 0.0669$ & 1.84 \\
\hline \hline
\end{tabular}

Table E.3: Amplitude extracted from $e+2$-jets $t \bar{t}$ candidates, with phase fixed appropriate to each coefficient case and assuming $d_{\mu \nu}=0$.

\begin{tabular}{ccc}
\hline \hline Coefficient Case & Amplitude \pm Uncertainty & Significance (sd) \\
\hline$X X$ & $-0.1288 \pm 0.0687$ & 1.88 \\
$X Y$ & $-0.0182 \pm 0.0701$ & 0.26 \\
$X Z$ & $0.0668 \pm 0.0660$ & 1.01 \\
$Y Z$ & $0.1690 \pm 0.0683$ & 2.48 \\
\hline \hline
\end{tabular}

Table E.4: Amplitude extracted from $e+2$-jets $t \bar{t}$ candidates, with phase fixed appropriate to each coefficient case and assuming $c_{\mu \nu}=0$.

\begin{tabular}{ccc}
\hline \hline Coefficient Case & Amplitude \pm Uncertainty & Significance (sd) \\
\hline$X X$ & $0.1288 \pm 0.0687$ & 1.88 \\
$X Y$ & $0.0182 \pm 0.0701$ & 0.26 \\
$X Z$ & $-0.1366 \pm 0.0675$ & 2.02 \\
$Y Z$ & $-0.1144 \pm 0.0667$ & 1.72 \\
\hline
\end{tabular}




\section{E.1.2 Muon amplitudes}

Table E.5: Amplitude extracted from $\mu+2$-jets $t \bar{t}$ candidates, with phase fixed appropriate to each coefficient case and assuming $\left(c_{R}\right)_{\mu \nu}=0$.

\begin{tabular}{ccc}
\hline \hline Coefficient Case & Amplitude \pm Uncertainty & Significance (sd) \\
\hline$X X$ & $0.0094 \pm 0.0822$ & 0.115 \\
$X Y$ & $0.0163 \pm 0.0839$ & 0.195 \\
$X Z$ & $0.0158 \pm 0.0808$ & 0.196 \\
$Y Z$ & $0.1214 \pm 0.0797$ & 1.52 \\
\hline
\end{tabular}

Table E.6: Amplitude extracted from $\mu+2$-jets $t \bar{t}$ candidates, with phase fixed appropriate to each coefficient case and assuming $\left(c_{L}\right)_{\mu \nu}=0$.

\begin{tabular}{ccc}
\hline \hline Coefficient Case & Amplitude \pm Uncertainty & Significance (sd) \\
\hline$X X$ & $-0.0094 \pm 0.0822$ & 0.115 \\
$X Y$ & $-0.0163 \pm 0.0839$ & 0.195 \\
$X Z$ & $-0.0006 \pm 0.0803$ & 0.00751 \\
$Y Z$ & $-0.1235 \pm 0.0802$ & 1.54 \\
\hline \hline
\end{tabular}

Table E.7: Amplitude extracted from $\mu+2$-jets $t \bar{t}$ candidates, with phase fixed appropriate to each coefficient case and assuming $d_{\mu \nu}=0$.

\begin{tabular}{ccc}
\hline \hline Coefficient Case & Amplitude \pm Uncertainty & Significance (sd) \\
\hline$X X$ & $-0.0094 \pm 0.0822$ & 0.115 \\
$X Y$ & $-0.0163 \pm 0.0839$ & 0.195 \\
$X Z$ & $0.1245 \pm 0.0808$ & 1.54 \\
$Y Z$ & $0.0170 \pm 0.0797$ & 0.213 \\
\hline \hline
\end{tabular}

Table E.8: Amplitude extracted from $\mu+2$-jets $t \bar{t}$ candidates, with phase fixed appropriate to each coefficient case and assuming $c_{\mu \nu}=0$.

\begin{tabular}{ccc}
\hline \hline Coefficient Case & Amplitude \pm Uncertainty & Significance (sd) \\
\hline$X X$ & $0.0094 \pm 0.0822$ & 0.115 \\
$X Y$ & $0.0163 \pm 0.0839$ & 0.195 \\
$X Z$ & $0.0083 \pm 0.0806$ & 0.103 \\
$Y Z$ & $0.1225 \pm 0.0799$ & 1.53 \\
\hline
\end{tabular}




\section{E.2 $\ell+3$-jets in Run IIa}

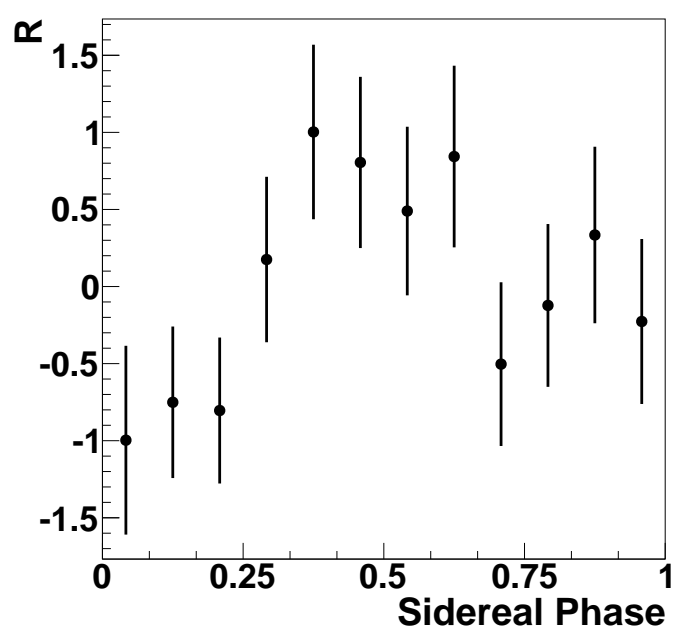

(a) $e+3$-jets $t \bar{t}$ candidates.

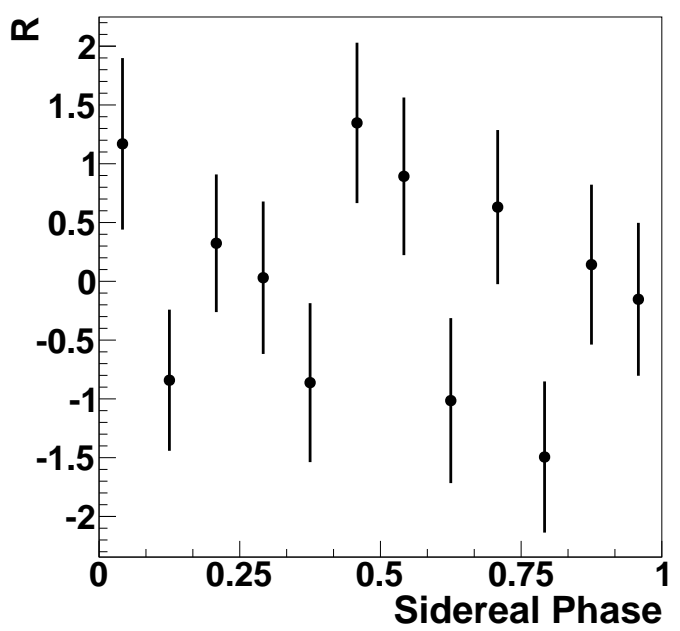

(b) $\mu+3$-jets $t \bar{t}$ candidates.

Figure E.2: Number of $\ell+3$-jets $t \bar{t}$ candidates per two sidereal hours, Run IIa. 


\section{E.2.1 Electron amplitudes}

Table E.9: Amplitude extracted from $e+3$-jets $t \bar{t}$ candidates, with phase fixed appropriate to each coefficient case and assuming $\left(c_{R}\right)_{\mu \nu}=0$.

\begin{tabular}{ccc}
\hline \hline Coefficient Case & Amplitude \pm Uncertainty & Significance (sd) \\
\hline$X X$ & $0.2366 \pm 0.1039$ & 2.27 \\
$X Y$ & $0.0026 \pm 0.1057$ & 0.0242 \\
$X Z$ & $0.1569 \pm 0.1022$ & 1.53 \\
$Y Z$ & $0.2682 \pm 0.1004$ & 2.67 \\
\hline \hline
\end{tabular}

Table E.10: Amplitude extracted from $e+3$-jets $t \bar{t}$ candidates, with phase fixed appropriate to each coefficient case and assuming $\left(c_{L}\right)_{\mu \nu}=0$.

\begin{tabular}{ccc}
\hline \hline Coefficient Case & Amplitude \pm Uncertainty & Significance (sd) \\
\hline$X X$ & $-0.2366 \pm 0.1039$ & 2.27 \\
$X Y$ & $-0.0026 \pm 0.1057$ & 0.0242 \\
$X Z$ & $-0.1144 \pm 0.1022$ & 1.12 \\
$Y Z$ & $-0.2904 \pm 0.1012$ & 2.87 \\
\hline \hline
\end{tabular}

Table E.11: Amplitude extracted from $e+3$-jets $t \bar{t}$ candidates, with phase fixed appropriate to each coefficient case and assuming $d_{\mu \nu}=0$.

\begin{tabular}{ccc}
\hline \hline Coefficient Case & Amplitude \pm Uncertainty & Significance (sd) \\
\hline$X X$ & $-0.2366 \pm 0.1039$ & 2.27 \\
$X Y$ & $-0.0026 \pm 0.1057$ & 0.0242 \\
$X Z$ & $0.0087 \pm 0.0998$ & 0.0876 \\
$Y Z$ & $-0.3249 \pm 0.1032$ & 3.14 \\
\hline \hline
\end{tabular}

Table E.12: Amplitude extracted from $e+3$-jets $t \bar{t}$ candidates, with phase fixed appropriate to each coefficient case and assuming $c_{\mu \nu}=0$.

\begin{tabular}{ccc}
\hline \hline Coefficient Case & Amplitude \pm Uncertainty & Significance (sd) \\
\hline$X X$ & $0.2366 \pm 0.1039$ & 2.27 \\
$X Y$ & $0.0026 \pm 0.1057$ & 0.0242 \\
$X Z$ & $0.1326 \pm 0.1022$ & 1.3 \\
$Y Z$ & $0.2813 \pm 0.1009$ & 2.79 \\
\hline
\end{tabular}




\section{E.2.2 Muon amplitudes}

Table E.13: Amplitude extracted from $\mu+3$-jets $t \bar{t}$ candidates, with phase fixed appropriate to each coefficient case and assuming $\left(c_{R}\right)_{\mu \nu}=0$.

\begin{tabular}{ccc}
\hline \hline Coefficient Case & Amplitude \pm Uncertainty & Significance $(\mathrm{sd})$ \\
\hline$X X$ & $0.0812 \pm 0.1235$ & 0.657 \\
$X Y$ & $0.2188 \pm 0.1256$ & 1.74 \\
$X Z$ & $0.1123 \pm 0.1214$ & 0.927 \\
$Y Z$ & $0.0160 \pm 0.1204$ & 0.134 \\
\hline
\end{tabular}

Table E.14: Amplitude extracted from $\mu+3$-jets $t \bar{t}$ candidates, with phase fixed appropriate to each coefficient case and assuming $\left(c_{L}\right)_{\mu \nu}=0$.

\begin{tabular}{ccc}
\hline \hline Coefficient Case & Amplitude \pm Uncertainty & Significance (sd) \\
\hline$X X$ & $-0.0812 \pm 0.1235$ & 0.657 \\
$X Y$ & $-0.2188 \pm 0.1256$ & 1.74 \\
$X Z$ & $-0.1083 \pm 0.1204$ & 0.898 \\
$Y Z$ & $-0.0296 \pm 0.1204$ & 0.245 \\
\hline \hline
\end{tabular}

Table E.15: Amplitude extracted from $\mu+3$-jets $t \bar{t}$ candidates, with phase fixed appropriate to each coefficient case and assuming $d_{\mu \nu}=0$.

\begin{tabular}{ccc}
\hline \hline Coefficient Case & Amplitude \pm Uncertainty & Significance (sd) \\
\hline$X X$ & $-0.0812 \pm 0.1235$ & 0.657 \\
$X Y$ & $-0.2188 \pm 0.1256$ & 1.74 \\
$X Z$ & $0.0454 \pm 0.1214$ & 0.374 \\
$Y Z$ & $-0.1022 \pm 0.1204$ & 0.848 \\
\hline \hline
\end{tabular}

Table E.16: Amplitude extracted from $\mu+3$-jets $t \bar{t}$ candidates, with phase fixed appropriate to each coefficient case and assuming $c_{\mu \nu}=0$.

\begin{tabular}{ccc}
\hline \hline Coefficient Case & Amplitude \pm Uncertainty & Significance (sd) \\
\hline$X X$ & $0.0812 \pm 0.1235$ & 0.657 \\
$X Y$ & $0.2188 \pm 0.1256$ & 1.74 \\
$X Z$ & $0.1103 \pm 0.1214$ & 0.914 \\
$Y Z$ & $0.0227 \pm 0.1204$ & 0.189 \\
\hline
\end{tabular}




\section{E.3 $\ell+>3$-jets in Run IIa}

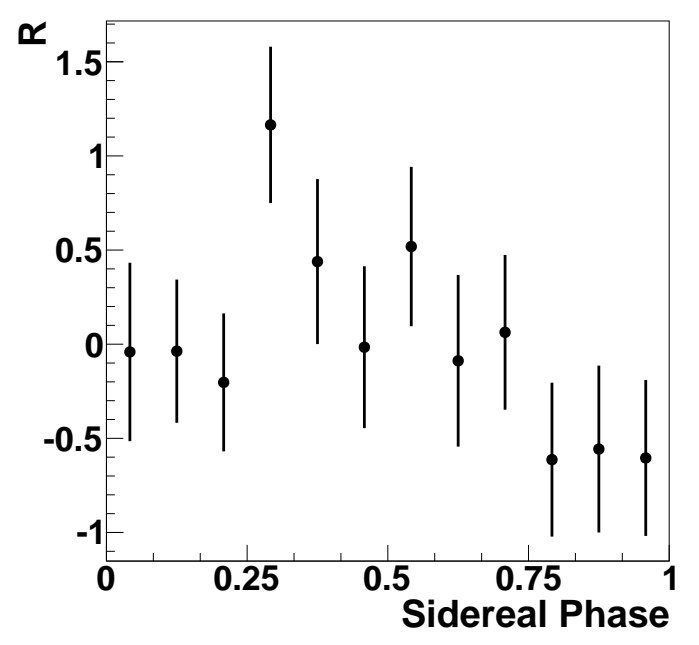

(a) $e+>3$-jets $t \bar{t}$ candidates.

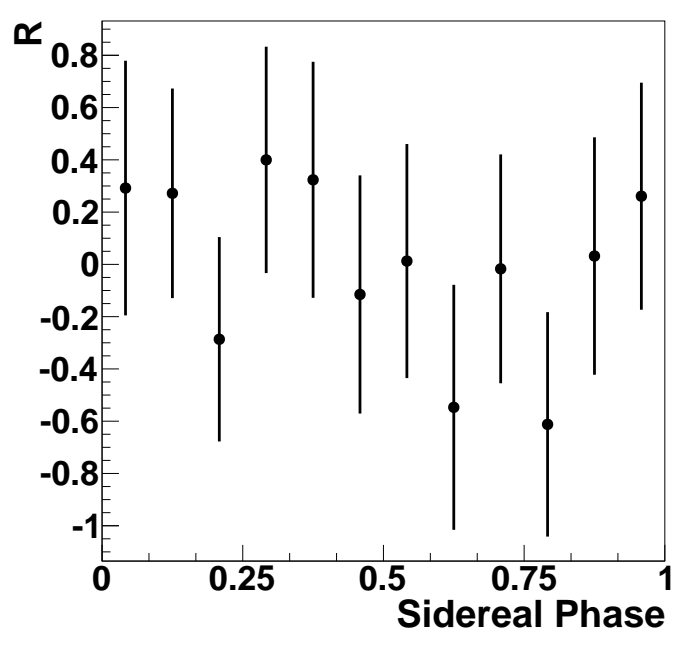

(b) $\mu+>3$-jets $t \bar{t}$ candidates.

Figure E.3: Number of $\ell+>3$-jets $t \bar{t}$ candidates per two sidereal hours, Run IIa. 


\section{E.3.1 Electron amplitudes}

Table E.17: Amplitude extracted from $e+>3$-jets $t \bar{t}$ candidates, with phase fixed appropriate to each coefficient case and assuming $\left(c_{R}\right)_{\mu \nu}=0$.

\begin{tabular}{ccc}
\hline \hline Coefficient Case & Amplitude \pm Uncertainty & Significance (sd) \\
\hline$X X$ & $-0.0470 \pm 0.1382$ & 0.34 \\
$X Y$ & $-0.0413 \pm 0.1413$ & 0.292 \\
$X Z$ & $0.4169 \pm 0.1366$ & 3.06 \\
$Y Z$ & $0.0259 \pm 0.1336$ & 0.194 \\
\hline
\end{tabular}

Table E.18: Amplitude extracted from $e+>3$-jets $t \bar{t}$ candidates, with phase fixed appropriate to each coefficient case and assuming $\left(c_{L}\right)_{\mu \nu}=0$.

\begin{tabular}{ccc}
\hline \hline Coefficient Case & Amplitude \pm Uncertainty & Significance (sd) \\
\hline$X X$ & $0.0470 \pm 0.1382$ & 0.34 \\
$X Y$ & $0.0413 \pm 0.1413$ & 0.292 \\
$X Z$ & $-0.4028 \pm 0.1356$ & 2.98 \\
$Y Z$ & $-0.0835 \pm 0.1346$ & 0.621 \\
\hline \hline
\end{tabular}

Table E.19: Amplitude extracted from $e+>3$-jets $t \bar{t}$ candidates, with phase fixed appropriate to each coefficient case and assuming $d_{\mu \nu}=0$.

\begin{tabular}{ccc}
\hline \hline Coefficient Case & Amplitude \pm Uncertainty & Significance (sd) \\
\hline$X X$ & $0.0470 \pm 0.1382$ & 0.34 \\
$X Y$ & $0.0413 \pm 0.1413$ & 0.292 \\
$X Z$ & $-0.3249 \pm 0.1326$ & 2.45 \\
$Y Z$ & $-0.2409 \pm 0.1376$ & 1.76 \\
\hline \hline
\end{tabular}

Table E.20: Amplitude extracted from $e+>3$-jets $t \bar{t}$ candidates, with phase fixed appropriate to each coefficient case and assuming $c_{\mu \nu}=0$.

\begin{tabular}{ccc}
\hline \hline Coefficient Case & Amplitude \pm Uncertainty & Significance (sd) \\
\hline$X X$ & $-0.0470 \pm 0.1382$ & 0.34 \\
$X Y$ & $-0.0413 \pm 0.1413$ & 0.292 \\
$X Z$ & $0.4099 \pm 0.1356$ & 3.02 \\
$Y Z$ & $0.0587 \pm 0.1346$ & 0.438 \\
\hline
\end{tabular}




\section{E.3.2 Muon amplitudes}

Table E.21: Amplitude extracted from $\mu+>3$-jets $t \bar{t}$ candidates, with phase fixed appropriate to each coefficient case and assuming $\left(c_{R}\right)_{\mu \nu}=0$.

\begin{tabular}{ccc}
\hline \hline Coefficient Case & Amplitude \pm Uncertainty & Significance $(\mathrm{sd})$ \\
\hline$X X$ & $0.1141 \pm 0.1403$ & 0.814 \\
$X Y$ & $0.0688 \pm 0.1434$ & 0.481 \\
$X Z$ & $0.0834 \pm 0.1376$ & 0.606 \\
$Y Z$ & $-0.1741 \pm 0.1356$ & 1.28 \\
\hline
\end{tabular}

Table E.22: Amplitude extracted from $\mu+>3$-jets $t \bar{t}$ candidates, with phase fixed appropriate to each coefficient case and assuming $\left(c_{L}\right)_{\mu \nu}=0$.

\begin{tabular}{ccc}
\hline \hline Coefficient Case & Amplitude \pm Uncertainty & Significance (sd) \\
\hline$X X$ & $-0.1141 \pm 0.1403$ & 0.814 \\
$X Y$ & $-0.0688 \pm 0.1434$ & 0.481 \\
$X Z$ & $-0.1032 \pm 0.1366$ & 0.754 \\
$Y Z$ & $0.1650 \pm 0.1366$ & 1.2 \\
\hline \hline
\end{tabular}

Table E.23: Amplitude extracted from $\mu+>3$-jets $t \bar{t}$ candidates, with phase fixed appropriate to each coefficient case and assuming $d_{\mu \nu}=0$.

\begin{tabular}{ccc}
\hline \hline Coefficient Case & Amplitude \pm Uncertainty & Significance (sd) \\
\hline$X X$ & $-0.1141 \pm 0.1403$ & 0.814 \\
$X Y$ & $-0.0688 \pm 0.1434$ & 0.481 \\
$X Z$ & $-0.1498 \pm 0.1376$ & 1.09 \\
$Y Z$ & $-0.1245 \pm 0.1356$ & 0.913 \\
\hline \hline
\end{tabular}

Table E.24: Amplitude extracted from $\mu+>3$-jets $t \bar{t}$ candidates, with phase fixed appropriate to each coefficient case and assuming $c_{\mu \nu}=0$.

\begin{tabular}{ccc}
\hline \hline Coefficient Case & Amplitude \pm Uncertainty & Significance (sd) \\
\hline$X X$ & $0.1141 \pm 0.1403$ & 0.814 \\
$X Y$ & $0.0688 \pm 0.1434$ & 0.481 \\
$X Z$ & $0.0934 \pm 0.1376$ & 0.68 \\
$Y Z$ & $-0.1700 \pm 0.1366$ & 1.24 \\
\hline
\end{tabular}




\section{E.4 $\ell+2$-jets in Run IIb}

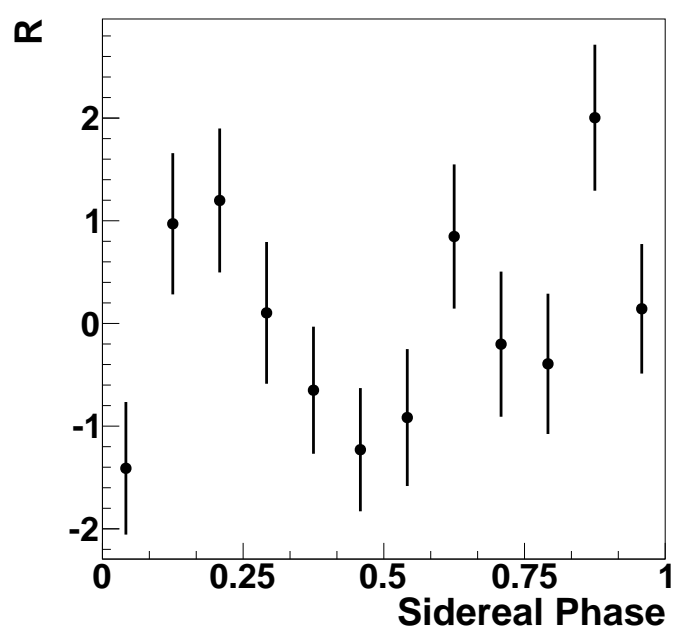

(a) $e+2$-jets $t \bar{t}$ candidates.

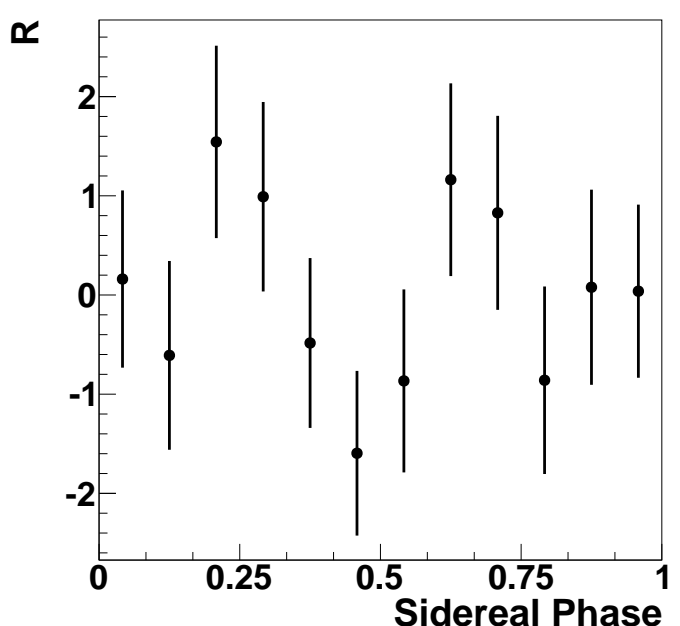

(b) $\mu+2$-jets $t \bar{t}$ candidates.

Figure E.4: Number of $\ell+2$-jets $t \bar{t}$ candidates per two sidereal hours, Run IIb. 


\section{E.4.1 Electron amplitudes}

Table E.25: Amplitude extracted from $e+2$-jets $t \bar{t}$ candidates, with phase fixed appropriate to each coefficient case and assuming $\left(c_{R}\right)_{\mu \nu}=0$.

\begin{tabular}{ccc}
\hline \hline Coefficient Case & Amplitude \pm Uncertainty & Significance $(\mathrm{sd})$ \\
\hline$X X$ & $-0.0566 \pm 0.0370$ & 1.53 \\
$X Y$ & $-0.0677 \pm 0.0372$ & 1.82 \\
$X Z$ & $-0.0552 \pm 0.0353$ & 1.56 \\
$Y Z$ & $-0.0426 \pm 0.0363$ & 1.17 \\
\hline
\end{tabular}

Table E.26: Amplitude extracted from $e+2$-jets $t \bar{t}$ candidates, with phase fixed appropriate to each coefficient case and assuming $\left(c_{L}\right)_{\mu \nu}=0$.

\begin{tabular}{ccc}
\hline \hline Coefficient Case & Amplitude \pm Uncertainty & Significance (sd) \\
\hline$X X$ & $0.0566 \pm 0.0370$ & 1.53 \\
$X Y$ & $0.0677 \pm 0.0372$ & 1.82 \\
$X Z$ & $0.0497 \pm 0.0356$ & 1.4 \\
$Y Z$ & $0.0496 \pm 0.0360$ & 1.38 \\
\hline \hline
\end{tabular}

Table E.27: Amplitude extracted from $e+2$-jets $t \bar{t}$ candidates, with phase fixed appropriate to each coefficient case and assuming $d_{\mu \nu}=0$.

\begin{tabular}{ccc}
\hline \hline Coefficient Case & Amplitude \pm Uncertainty & Significance (sd) \\
\hline$X X$ & $0.0566 \pm 0.0370$ & 1.53 \\
$X Y$ & $0.0677 \pm 0.0372$ & 1.82 \\
$X Z$ & $0.0283 \pm 0.0364$ & 0.776 \\
$Y Z$ & $0.0624 \pm 0.0352$ & 1.77 \\
\hline \hline
\end{tabular}

Table E.28: Amplitude extracted from $e+2$-jets $t \bar{t}$ candidates, with phase fixed appropriate to each coefficient case and assuming $c_{\mu \nu}=0$.

\begin{tabular}{ccc}
\hline \hline Coefficient Case & Amplitude \pm Uncertainty & Significance (sd) \\
\hline$X X$ & $-0.0566 \pm 0.0370$ & 1.53 \\
$X Y$ & $-0.0677 \pm 0.0372$ & 1.82 \\
$X Z$ & $-0.0522 \pm 0.0355$ & 1.47 \\
$Y Z$ & $-0.0468 \pm 0.0362$ & 1.29 \\
\hline
\end{tabular}




\section{E.4.2 Muon amplitudes}

Table E.29: Amplitude extracted from $\mu+2$-jets $t \bar{t}$ candidates, with phase fixed appropriate to each coefficient case and assuming $\left(c_{R}\right)_{\mu \nu}=0$.

\begin{tabular}{ccc}
\hline \hline Coefficient Case & Amplitude \pm Uncertainty & Significance $(\mathrm{sd})$ \\
\hline$X X$ & $-0.0836 \pm 0.0437$ & 1.91 \\
$X Y$ & $-0.0467 \pm 0.0439$ & 1.06 \\
$X Z$ & $-0.0176 \pm 0.0418$ & 0.422 \\
$Y Z$ & $-0.0343 \pm 0.0429$ & 0.798 \\
\hline \hline
\end{tabular}

Table E.30: Amplitude extracted from $\mu+2$-jets $t \bar{t}$ candidates, with phase fixed appropriate to each coefficient case and assuming $\left(c_{L}\right)_{\mu \nu}=0$.

\begin{tabular}{ccc}
\hline \hline Coefficient Case & Amplitude \pm Uncertainty & Significance (sd) \\
\hline$X X$ & $0.0836 \pm 0.0437$ & 1.91 \\
$X Y$ & $0.0467 \pm 0.0439$ & 1.06 \\
$X Z$ & $0.0138 \pm 0.0421$ & 0.328 \\
$Y Z$ & $0.0357 \pm 0.0426$ & 0.839 \\
\hline \hline
\end{tabular}

Table E.31: Amplitude extracted from $\mu+2$-jets $t \bar{t}$ candidates, with phase fixed appropriate to each coefficient case and assuming $d_{\mu \nu}=0$.

\begin{tabular}{ccc}
\hline \hline Coefficient Case & Amplitude \pm Uncertainty & Significance (sd) \\
\hline$X X$ & $0.0836 \pm 0.0437$ & 1.91 \\
$X Y$ & $0.0467 \pm 0.0439$ & 1.06 \\
$X Z$ & $-0.0367 \pm 0.0422$ & 0.87 \\
$Y Z$ & $0.0088 \pm 0.0425$ & 0.207 \\
\hline \hline
\end{tabular}

Table E.32: Amplitude extracted from $\mu+2$-jets $t \bar{t}$ candidates, with phase fixed appropriate to each coefficient case and assuming $c_{\mu \nu}=0$.

\begin{tabular}{ccc}
\hline \hline Coefficient Case & Amplitude \pm Uncertainty & Significance (sd) \\
\hline$X X$ & $-0.0836 \pm 0.0437$ & 1.91 \\
$X Y$ & $-0.0467 \pm 0.0439$ & 1.06 \\
$X Z$ & $-0.0158 \pm 0.0419$ & 0.376 \\
$Y Z$ & $-0.0350 \pm 0.0428$ & 0.82 \\
\hline
\end{tabular}




\section{E.5 $\ell+3$-jets in Run IIb}

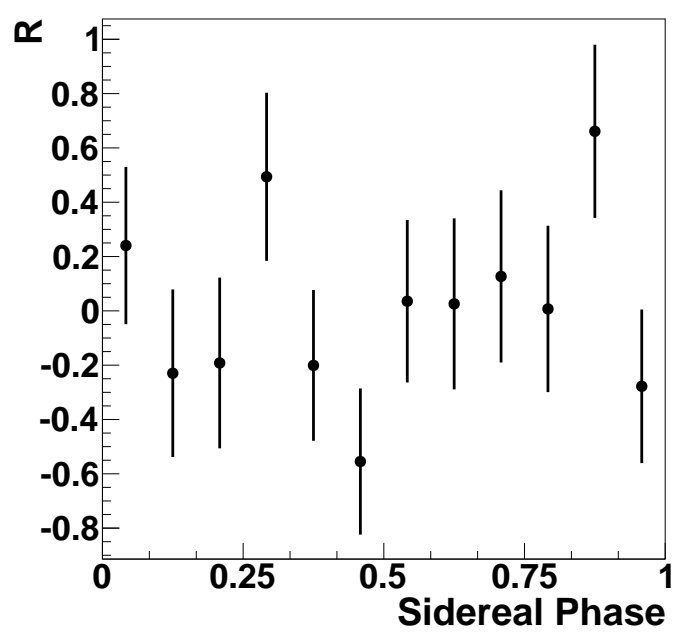

(a) $e+3$-jets $t \bar{t}$ candidates.

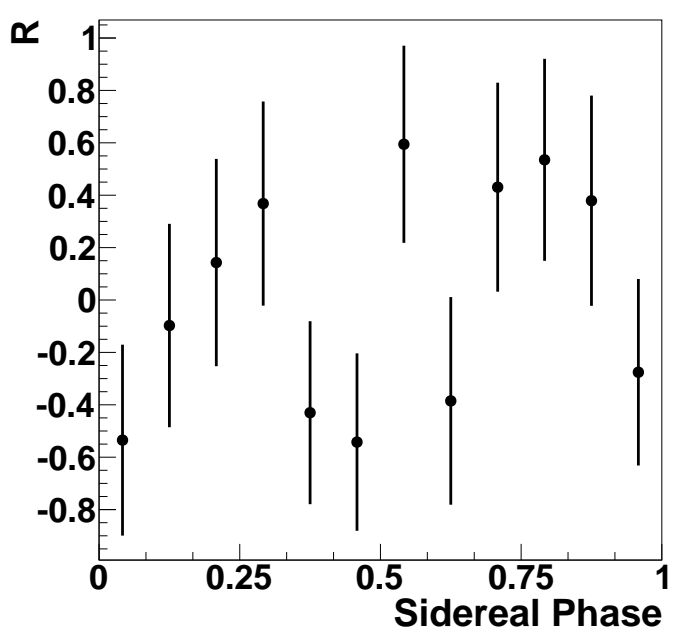

(b) $\mu+3$-jets $t \bar{t}$ candidates.

Figure E.5: Number of $\ell+3$-jets $t \bar{t}$ candidates per two sidereal hours, Run IIb. 


\section{E.5.1 Electron amplitudes}

Table E.33: Amplitude extracted from $e+3$-jets $t \bar{t}$ candidates, with phase fixed appropriate to each coefficient case and assuming $\left(c_{R}\right)_{\mu \nu}=0$.

\begin{tabular}{ccc}
\hline \hline Coefficient Case & Amplitude \pm Uncertainty & Significance (sd) \\
\hline$X X$ & $-0.0261 \pm 0.0579$ & 0.451 \\
$X Y$ & $-0.0731 \pm 0.0582$ & 1.26 \\
$X Z$ & $-0.0742 \pm 0.0554$ & 1.34 \\
$Y Z$ & $-0.0116 \pm 0.0570$ & 0.204 \\
\hline
\end{tabular}

Table E.34: Amplitude extracted from $e+3$-jets $t \bar{t}$ candidates, with phase fixed appropriate to each coefficient case and assuming $\left(c_{L}\right)_{\mu \nu}=0$.

\begin{tabular}{ccc}
\hline \hline Coefficient Case & Amplitude \pm Uncertainty & Significance (sd) \\
\hline$X X$ & $0.0261 \pm 0.0579$ & 0.451 \\
$X Y$ & $0.0731 \pm 0.0582$ & 1.26 \\
$X Z$ & $0.0731 \pm 0.0558$ & 1.31 \\
$Y Z$ & $0.0223 \pm 0.0565$ & 0.394 \\
\hline \hline
\end{tabular}

Table E.35: Amplitude extracted from $e+3$-jets $t \bar{t}$ candidates, with phase fixed appropriate to each coefficient case and assuming $d_{\mu \nu}=0$.

\begin{tabular}{ccc}
\hline \hline Coefficient Case & Amplitude \pm Uncertainty & Significance (sd) \\
\hline$X X$ & $0.0261 \pm 0.0579$ & 0.451 \\
$X Y$ & $0.0731 \pm 0.0582$ & 1.26 \\
$X Z$ & $0.0618 \pm 0.0572$ & 1.08 \\
$Y Z$ & $0.0474 \pm 0.0552$ & 0.859 \\
\hline \hline
\end{tabular}

Table E.36: Amplitude extracted from $e+3$-jets $t \bar{t}$ candidates, with phase fixed appropriate to each coefficient case and assuming $c_{\mu \nu}=0$.

\begin{tabular}{ccc}
\hline \hline Coefficient Case & Amplitude \pm Uncertainty & Significance (sd) \\
\hline$X X$ & $-0.0261 \pm 0.0579$ & 0.451 \\
$X Y$ & $-0.0731 \pm 0.0582$ & 1.26 \\
$X Z$ & $-0.0738 \pm 0.0556$ & 1.33 \\
$Y Z$ & $-0.0178 \pm 0.0567$ & 0.314 \\
\hline
\end{tabular}




\section{E.5.2 Muon amplitudes}

Table E.37: Amplitude extracted from $\mu+3$-jets $t \bar{t}$ candidates, with phase fixed appropriate to each coefficient case and assuming $\left(c_{R}\right)_{\mu \nu}=0$.

\begin{tabular}{ccc}
\hline \hline Coefficient Case & Amplitude \pm Uncertainty & Significance (sd) \\
\hline$X X$ & $-0.0801 \pm 0.0714$ & 1.12 \\
$X Y$ & $-0.1351 \pm 0.0718$ & 1.87 \\
$X Z$ & $-0.0642 \pm 0.0683$ & 0.939 \\
$Y Z$ & $0.0644 \pm 0.0702$ & 0.917 \\
\hline
\end{tabular}

Table E.38: Amplitude extracted from $\mu+3$-jets $t \bar{t}$ candidates, with phase fixed appropriate to each coefficient case and assuming $\left(c_{L}\right)_{\mu \nu}=0$.

\begin{tabular}{ccc}
\hline \hline Coefficient Case & Amplitude \pm Uncertainty & Significance (sd) \\
\hline$X X$ & $0.0801 \pm 0.0714$ & 1.12 \\
$X Y$ & $0.1351 \pm 0.0718$ & 1.87 \\
$X Z$ & $0.0721 \pm 0.0688$ & 1.05 \\
$Y Z$ & $-0.0549 \pm 0.0696$ & 0.787 \\
\hline \hline
\end{tabular}

Table E.39: Amplitude extracted from $\mu+3$-jets $t \bar{t}$ candidates, with phase fixed appropriate to each coefficient case and assuming $d_{\mu \nu}=0$.

\begin{tabular}{ccc}
\hline \hline Coefficient Case & Amplitude \pm Uncertainty & Significance (sd) \\
\hline$X X$ & $0.0801 \pm 0.0714$ & 1.12 \\
$X Y$ & $0.1351 \pm 0.0718$ & 1.87 \\
$X Z$ & $0.0429 \pm 0.0690$ & 0.621 \\
$Y Z$ & $0.0805 \pm 0.0694$ & 1.16 \\
\hline \hline
\end{tabular}

Table E.40: Amplitude extracted from $\mu+3$-jets $t \bar{t}$ candidates, with phase fixed appropriate to each coefficient case and assuming $c_{\mu \nu}=0$.

\begin{tabular}{ccc}
\hline \hline Coefficient Case & Amplitude \pm Uncertainty & Significance (sd) \\
\hline$X X$ & $-0.0801 \pm 0.0714$ & 1.12 \\
$X Y$ & $-0.1351 \pm 0.0718$ & 1.87 \\
$X Z$ & $-0.0682 \pm 0.0685$ & 0.994 \\
$Y Z$ & $0.0597 \pm 0.0699$ & 0.854 \\
\hline
\end{tabular}




\section{E.6 $\ell+>3$-jets in Run IIb}

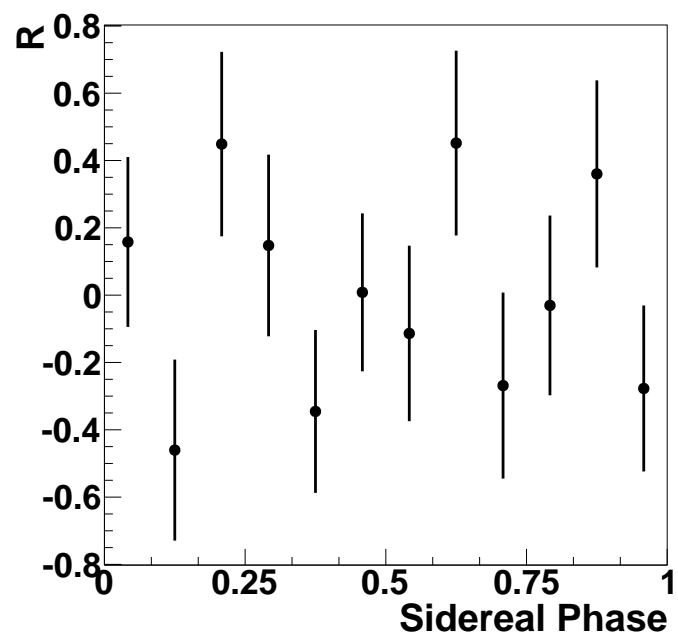

(a) $e+>3$-jets $t \bar{t}$ candidates.

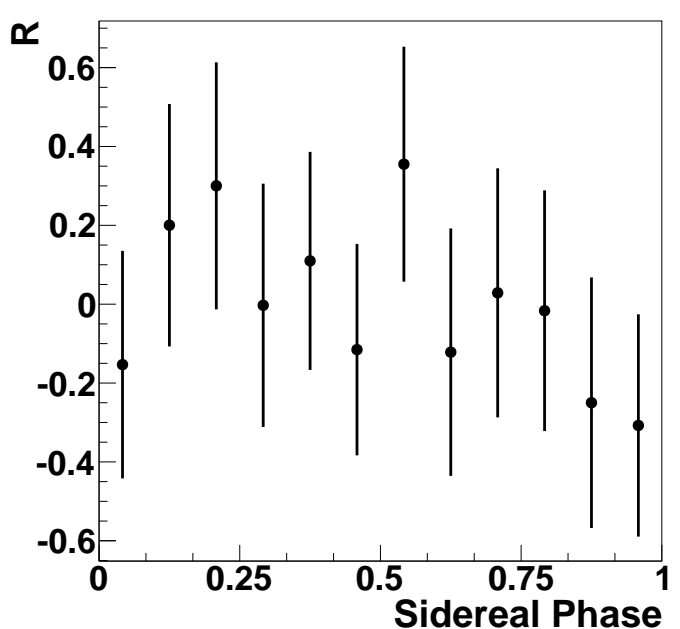

(b) $\mu+>3$-jets $t \bar{t}$ candidates.

Figure E.6: Number of $\ell+>3$-jets $t \bar{t}$ candidates per two sidereal hours, Run IIb. 


\section{E.6.1 Electron amplitudes}

Table E.41: Amplitude extracted from $e+>3$-jets $t \bar{t}$ candidates, with phase fixed appropriate to each coefficient case and assuming $\left(c_{R}\right)_{\mu \nu}=0$.

\begin{tabular}{ccc}
\hline \hline Coefficient Case & Amplitude \pm Uncertainty & Significance (sd) \\
\hline$X X$ & $-0.0614 \pm 0.0868$ & 0.707 \\
$X Y$ & $-0.0360 \pm 0.0872$ & 0.413 \\
$X Z$ & $-0.0151 \pm 0.0829$ & 0.182 \\
$Y Z$ & $0.0150 \pm 0.0853$ & 0.175 \\
\hline
\end{tabular}

Table E.42: Amplitude extracted from $e+>3$-jets $t \bar{t}$ candidates, with phase fixed appropriate to each coefficient case and assuming $\left(c_{L}\right)_{\mu \nu}=0$.

\begin{tabular}{ccc}
\hline \hline Coefficient Case & Amplitude \pm Uncertainty & Significance (sd) \\
\hline$X X$ & $0.0614 \pm 0.0868$ & 0.707 \\
$X Y$ & $0.0360 \pm 0.0872$ & 0.413 \\
$X Z$ & $0.0172 \pm 0.0835$ & 0.206 \\
$Y Z$ & $-0.0123 \pm 0.0846$ & 0.146 \\
\hline \hline
\end{tabular}

Table E.43: Amplitude extracted from $e+>3$-jets $t \bar{t}$ candidates, with phase fixed appropriate to each coefficient case and assuming $d_{\mu \nu}=0$.

\begin{tabular}{ccc}
\hline \hline Coefficient Case & Amplitude \pm Uncertainty & Significance $(\mathrm{sd})$ \\
\hline$X X$ & $0.0614 \pm 0.0868$ & 0.707 \\
$X Y$ & $0.0360 \pm 0.0872$ & 0.413 \\
$X Z$ & $0.0216 \pm 0.0856$ & 0.252 \\
$Y Z$ & $-0.0043 \pm 0.0826$ & 0.0518 \\
\hline \hline
\end{tabular}

Table E.44: Amplitude extracted from $e+>3$-jets $t \bar{t}$ candidates, with phase fixed appropriate to each coefficient case and assuming $c_{\mu \nu}=0$.

\begin{tabular}{ccc}
\hline \hline Coefficient Case & Amplitude \pm Uncertainty & Significance (sd) \\
\hline$X X$ & $-0.0614 \pm 0.0868$ & 0.707 \\
$X Y$ & $-0.0360 \pm 0.0872$ & 0.413 \\
$X Z$ & $-0.0164 \pm 0.0832$ & 0.196 \\
$Y Z$ & $0.0135 \pm 0.0849$ & 0.159 \\
\hline
\end{tabular}




\section{E.6.2 Muon amplitudes}

Table E.45: Amplitude extracted from $\mu+>3$-jets $t \bar{t}$ candidates, with phase fixed appropriate to each coefficient case and assuming $\left(c_{R}\right)_{\mu \nu}=0$.

\begin{tabular}{ccc}
\hline \hline Coefficient Case & Amplitude \pm Uncertainty & Significance (sd) \\
\hline$X X$ & $-0.1078 \pm 0.0960$ & 1.13 \\
$X Y$ & $-0.0139 \pm 0.0964$ & 0.145 \\
$X Z$ & $0.1133 \pm 0.0918$ & 1.23 \\
$Y Z$ & $0.0102 \pm 0.0943$ & 0.108 \\
\hline
\end{tabular}

Table E.46: Amplitude extracted from $\mu+>3$-jets $t \bar{t}$ candidates, with phase fixed appropriate to each coefficient case and assuming $\left(c_{L}\right)_{\mu \nu}=0$.

\begin{tabular}{ccc}
\hline \hline Coefficient Case & Amplitude \pm Uncertainty & Significance (sd) \\
\hline$X X$ & $0.1078 \pm 0.0960$ & 1.13 \\
$X Y$ & $0.0139 \pm 0.0964$ & 0.145 \\
$X Z$ & $-0.1133 \pm 0.0924$ & 1.22 \\
$Y Z$ & $-0.0243 \pm 0.0936$ & 0.259 \\
\hline \hline
\end{tabular}

Table E.47: Amplitude extracted from $\mu+>3$-jets $t \bar{t}$ candidates, with phase fixed appropriate to each coefficient case and assuming $d_{\mu \nu}=0$.

\begin{tabular}{ccc}
\hline \hline Coefficient Case & Amplitude \pm Uncertainty & Significance (sd) \\
\hline$X X$ & $0.1078 \pm 0.0960$ & 1.13 \\
$X Y$ & $0.0139 \pm 0.0964$ & 0.145 \\
$X Z$ & $0.0399 \pm 0.0928$ & 0.43 \\
$Y Z$ & $-0.1103 \pm 0.0933$ & 1.18 \\
\hline \hline
\end{tabular}

Table E.48: Amplitude extracted from $\mu+>3$-jets $t \bar{t}$ candidates, with phase fixed appropriate to each coefficient case and assuming $c_{\mu \nu}=0$.

\begin{tabular}{ccc}
\hline \hline Coefficient Case & Amplitude \pm Uncertainty & Significance (sd) \\
\hline$X X$ & $-0.1078 \pm 0.0960$ & 1.13 \\
$X Y$ & $-0.0139 \pm 0.0964$ & 0.145 \\
$X Z$ & $0.1133 \pm 0.0921$ & 1.23 \\
$Y Z$ & $0.0172 \pm 0.0939$ & 0.183 \\
\hline \hline
\end{tabular}




\section{E.7 Agreement across samples}

Table E.49: The values of $\chi^{2}$ and probability reflecting consistency with no time dependence for the 12 independent measurements of amplitudes of sidereal oscillations in all channels $(\ell+n$-jets, where $\ell=e, \mu$ and $n=2,3, \geq 4$ divided into Run IIa and Run IIb) for each of the 16 phases assumed in this analysis.

\begin{tabular}{llll}
\hline \hline Phase Assumption & $\chi^{2}(12$ dof $)$ & $\operatorname{Prob}\left(\chi^{2}\right)$ \\
\hline$\left(c_{R}\right)_{\mu \nu}=0$ & $X X$ & 19.162 & 0.085 \\
& $X Y$ & 13.213 & 0.354 \\
& $X Z$ & 24.466 & 0.018 \\
& $Y Z$ & 16.467 & 0.171 \\
\hline$\left(c_{L}\right)_{\mu \nu}=0$ & $X X$ & 19.162 & 0.085 \\
& $X Y$ & 13.213 & 0.354 \\
& $X Z$ & 21.456 & 0.044 \\
& $Y Z$ & 19.381 & 0.080 \\
\hline$d_{\mu \nu}=0$ & $X X$ & 19.162 & 0.085 \\
& $X Y$ & 13.213 & 0.354 \\
& $X Z$ & 13.903 & 0.307 \\
& $Y Z$ & 27.363 & 0.007 \\
\hline$c_{\mu \nu}=0$ & $X X$ & 19.162 & 0.085 \\
& $X Y$ & 13.213 & 0.354 \\
& $X Z$ & 22.821 & 0.029 \\
& $Y Z$ & 18.056 & 0.114 \\
\hline \hline \multicolumn{4}{c}{}
\end{tabular}


Appendix F

\section{Data and Fits}




\section{F.1 $e+>$ 3-jets}
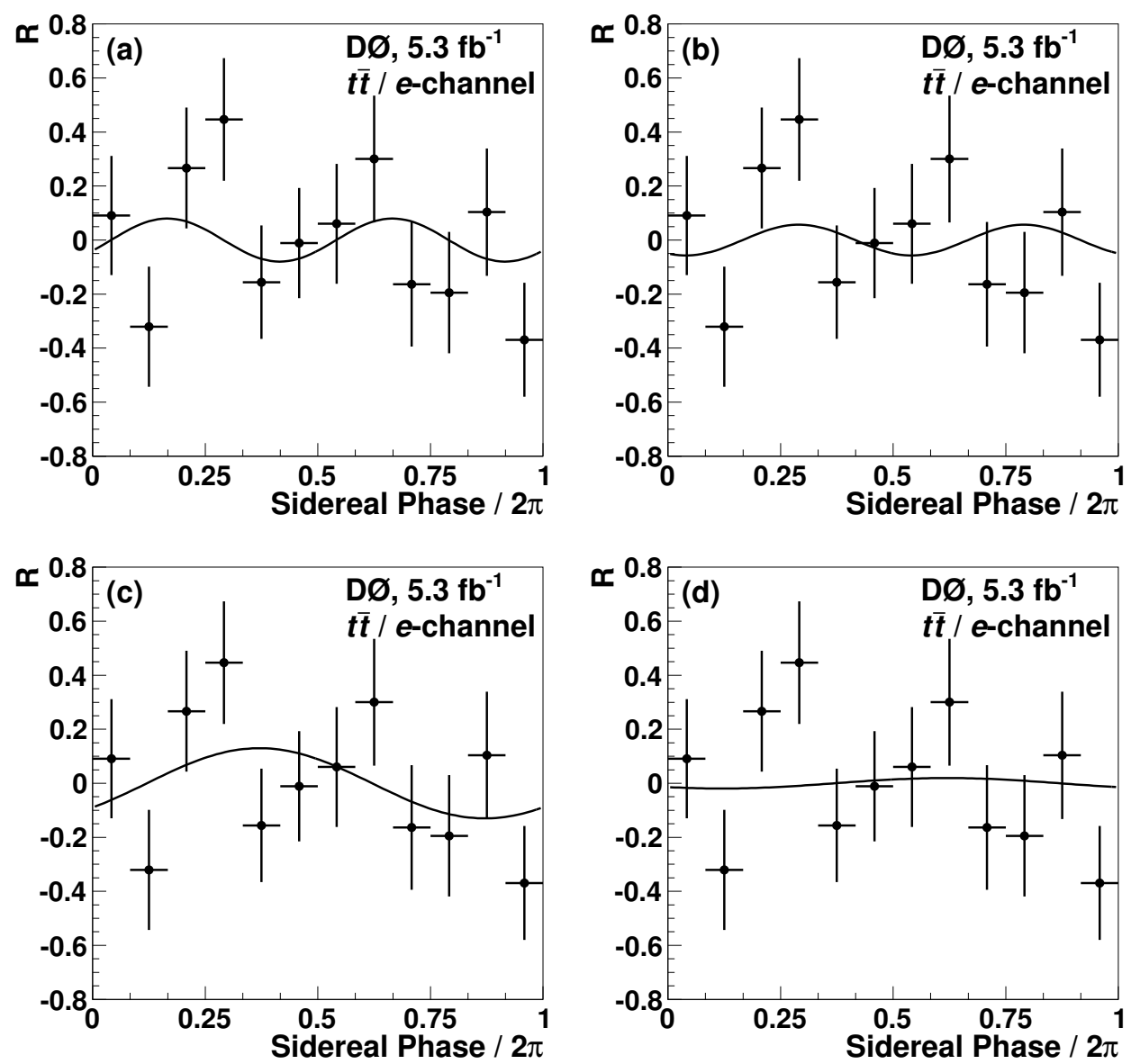

Figure F.1: Fitted $f_{\mathrm{SME}}\left(\phi_{i}\right)$ (line) and observed $R_{i}$ (crosses), $e+>3$-jets $t \bar{t}$ candidates, for $\left(c_{R}\right)_{\mu \nu}=0$ : (a) $\left(c_{L}\right)_{X X}$, (b) $\left(c_{L}\right)_{X Y}$, (c) $\left(c_{L}\right)_{X Z}$, (d) $\left(c_{L}\right)_{Y Z}$. 

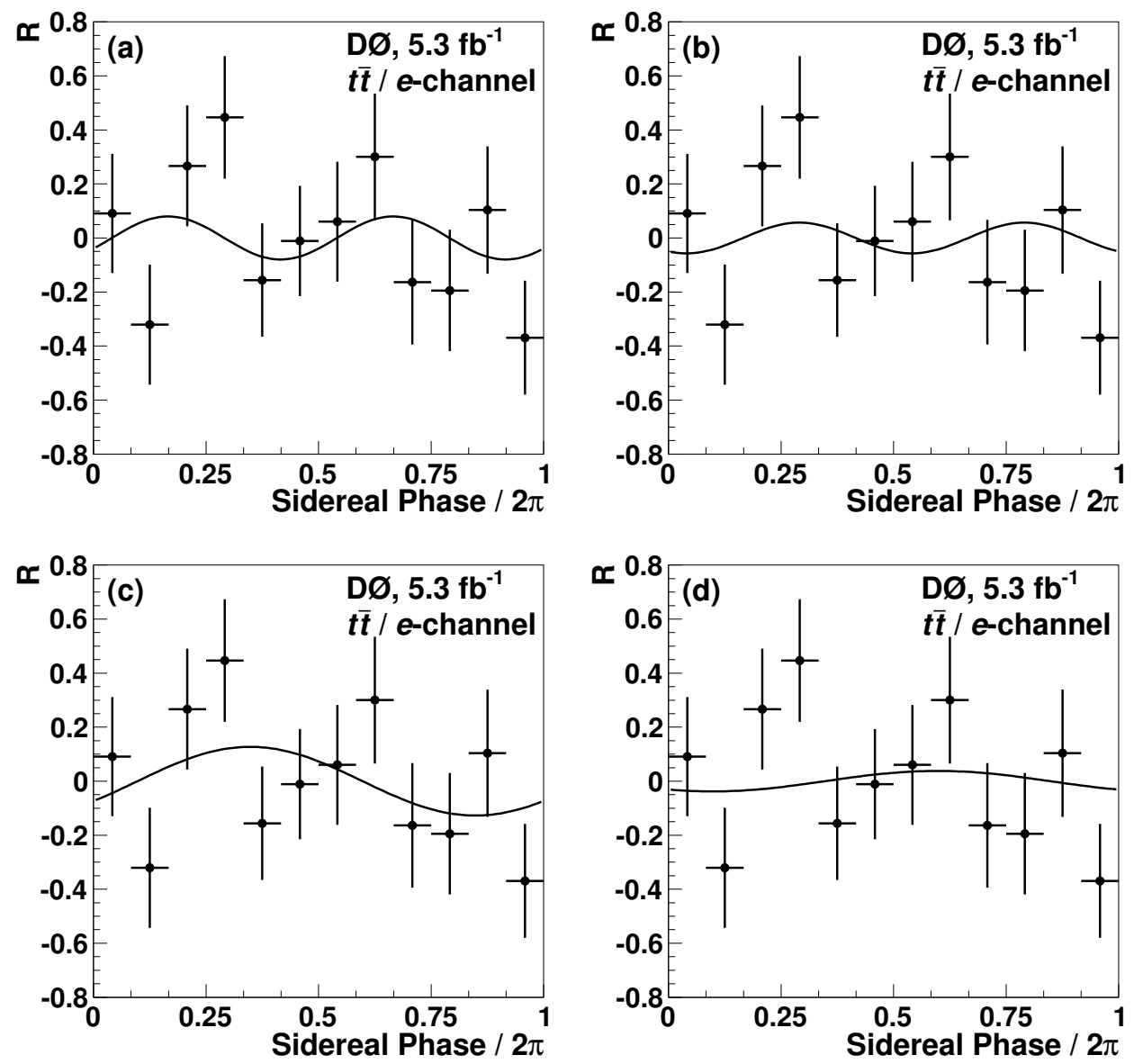

Figure F.2: Fitted $f_{\mathrm{SME}}\left(\phi_{i}\right)$ (line) and observed $R_{i}$ (crosses), $e+>3$-jets $t \bar{t}$ candidates, for $\left(c_{L}\right)_{\mu \nu}=0$ : (a) $\left(c_{R}\right)_{X X}$, (b) $\left(c_{R}\right)_{X Y}$, (c) $\left(c_{R}\right)_{X Z}$, (d) $\left(c_{R}\right)_{Y Z}$. 

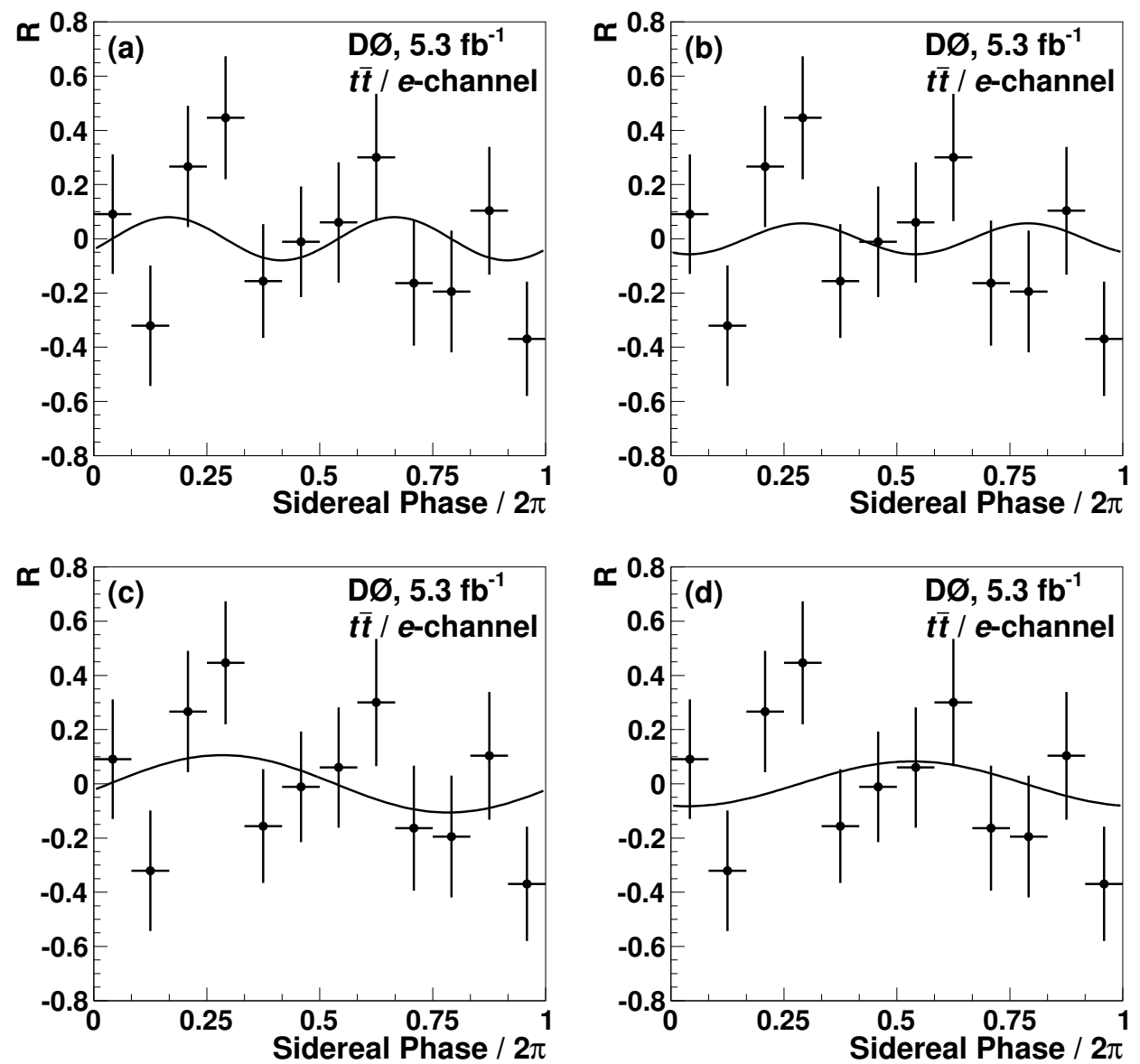

Figure F.3: Fitted $f_{\mathrm{SME}}\left(\phi_{i}\right)$ (line) and observed $R_{i}$ (crosses), $e+>3$-jets $t \bar{t}$ candidates, for $d_{\mu \nu}=0$ : (a) $c_{X X}$, (b) $c_{X Y}$, (c) $c_{X Z}$, (d) $c_{Y Z}$. 

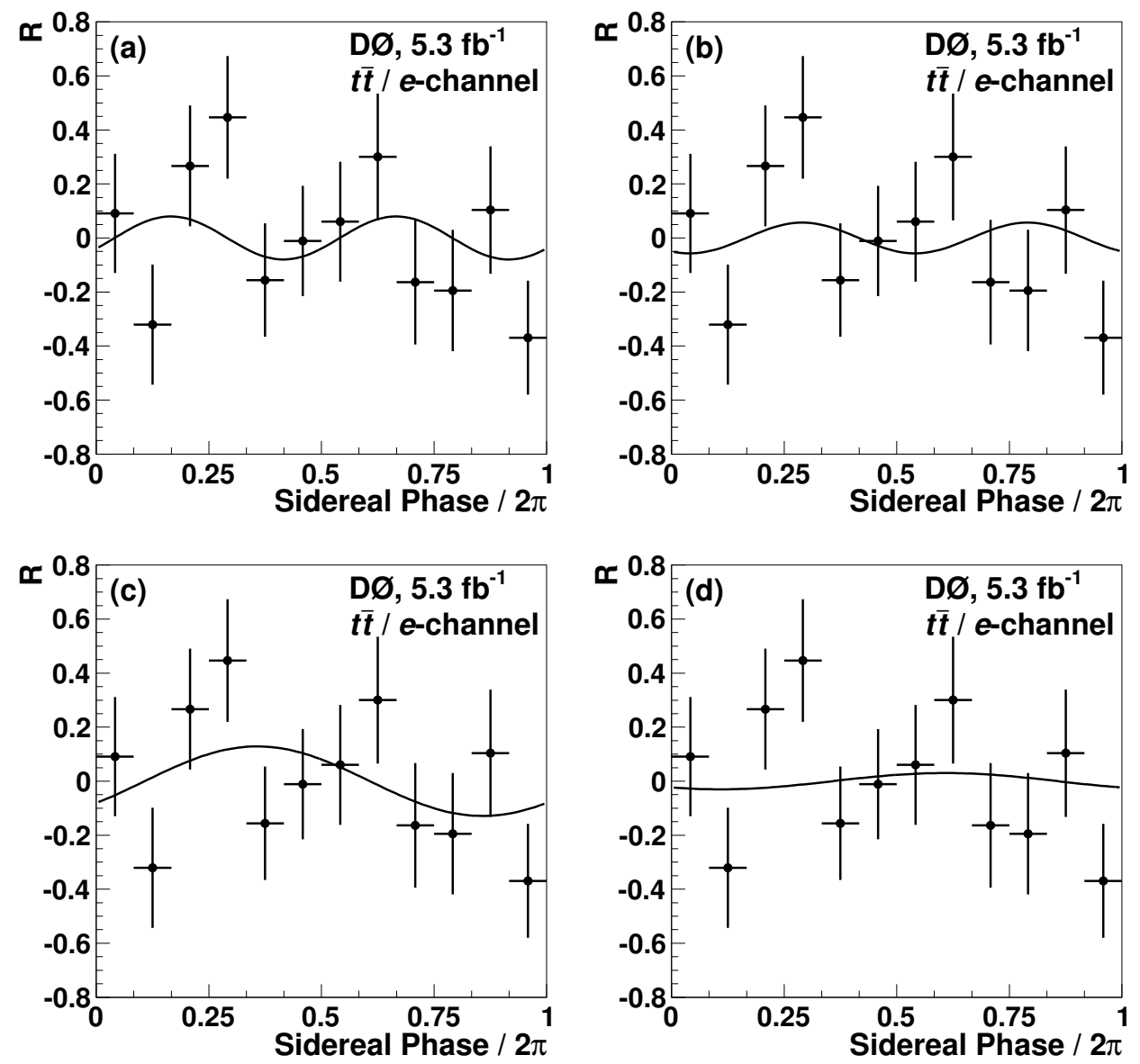

Figure F.4: Fitted $f_{\mathrm{SME}}\left(\phi_{i}\right)$ (line) and observed $R_{i}$ (crosses), $e+>3$-jets $t \bar{t}$ candidates, for $c_{\mu \nu}=0$ : (a) $d_{X X}$, (b) $d_{X Y}$, (c) $d_{X Z}$, (d) $d_{Y Z}$. 
F.2 $\mu+>3$-jets
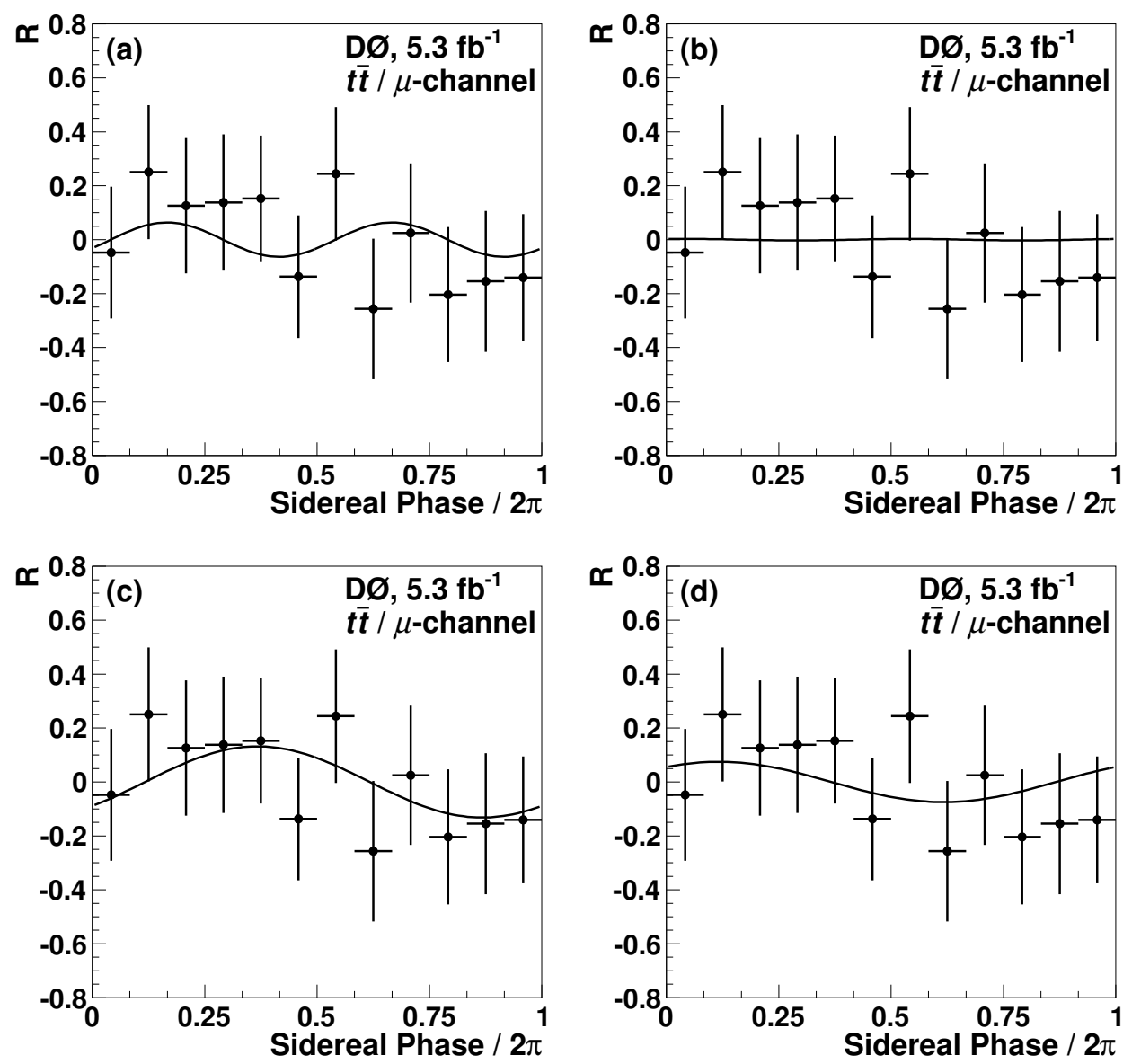

Figure F.5: Fitted $f_{\mathrm{SME}}\left(\phi_{i}\right)$ (line) and observed $R_{i}$ (crosses), $\mu+>3$-jets $t \bar{t}$ candidates, for $\left(c_{R}\right)_{\mu \nu}=0$ : (a) $\left(c_{L}\right)_{X X}$, (b) $\left(c_{L}\right)_{X Y}$, (c) $\left(c_{L}\right)_{X Z}$, (d) $\left(c_{L}\right)_{Y Z}$. 

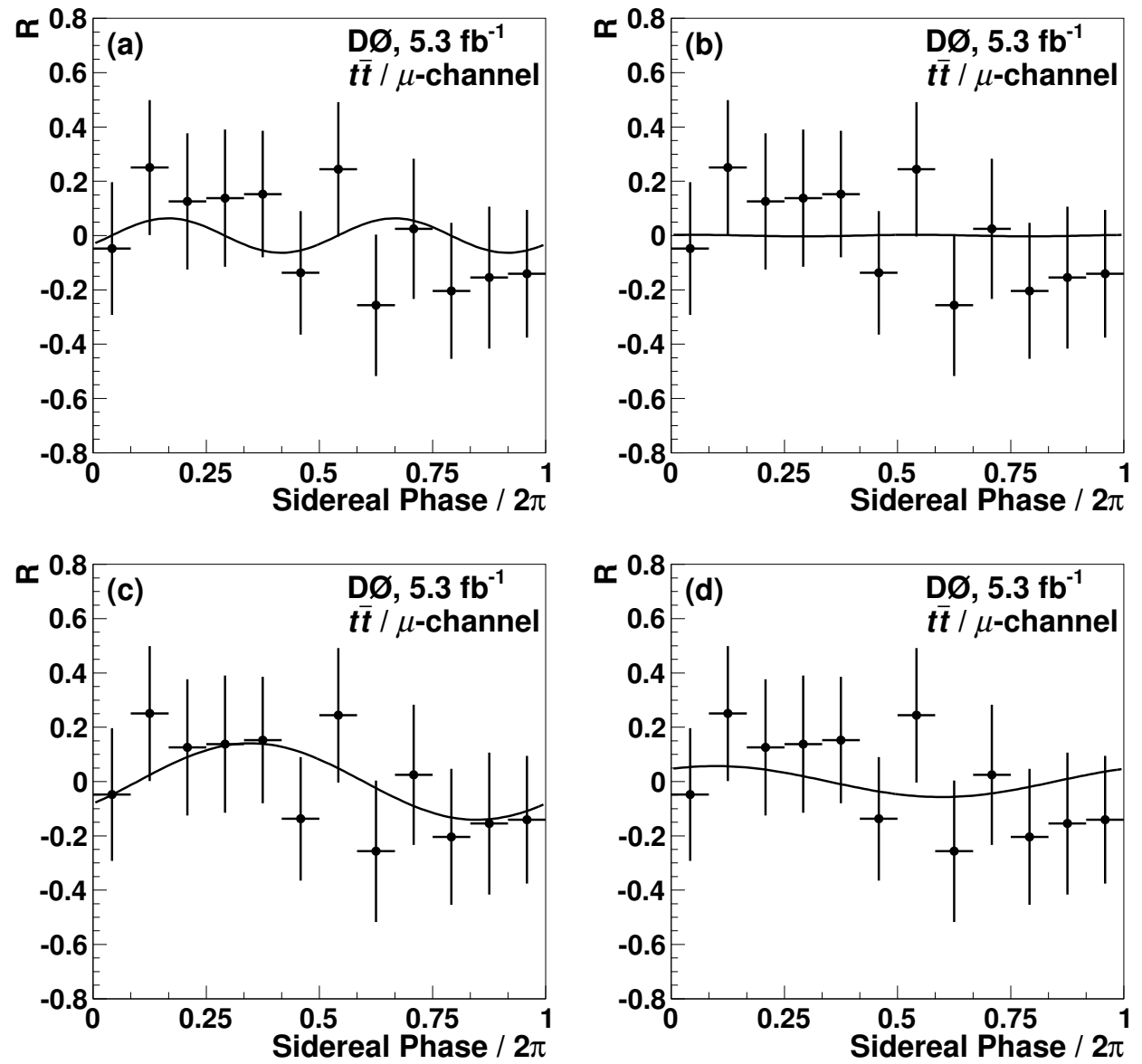

Figure F.6: Fitted $f_{\mathrm{SME}}\left(\phi_{i}\right)$ (line) and observed $R_{i}$ (crosses), $\mu+>3$-jets $t \bar{t}$ candidates, for $\left(c_{L}\right)_{\mu \nu}=0$ : (a) $\left(c_{R}\right)_{X X}$, (b) $\left(c_{R}\right)_{X Y}$, (c) $\left(c_{R}\right)_{X Z}$, (d) $\left(c_{R}\right)_{Y Z}$. 

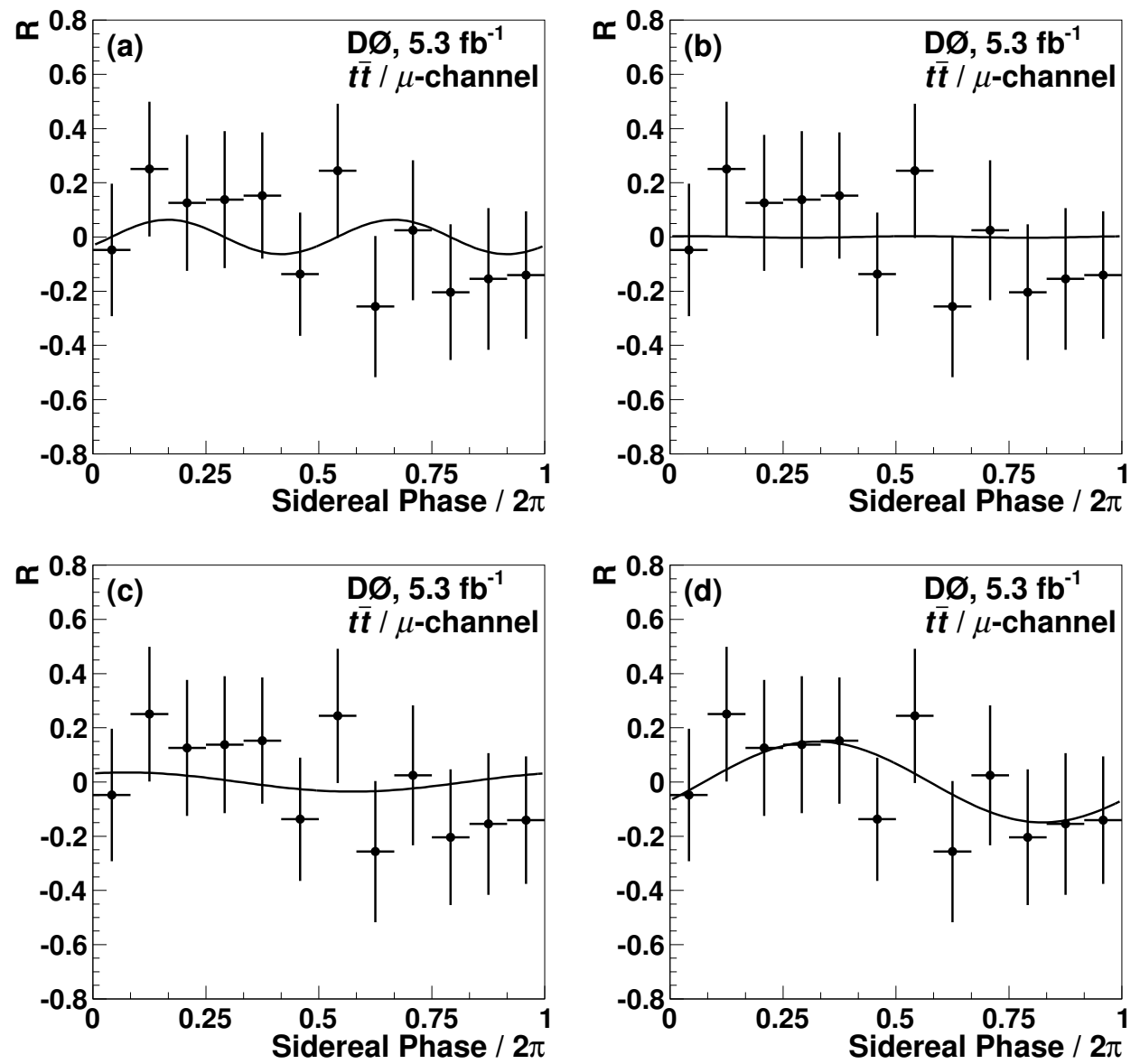

Figure F.7: Fitted $f_{\mathrm{SME}}\left(\phi_{i}\right)$ (line) and observed $R_{i}$ (crosses), $\mu+>3$-jets $t \bar{t}$ candidates, for $d_{\mu \nu}=0$ : (a) $c_{X X}$, (b) $c_{X Y}$, (c) $c_{X Z}$, (d) $c_{Y Z}$. 

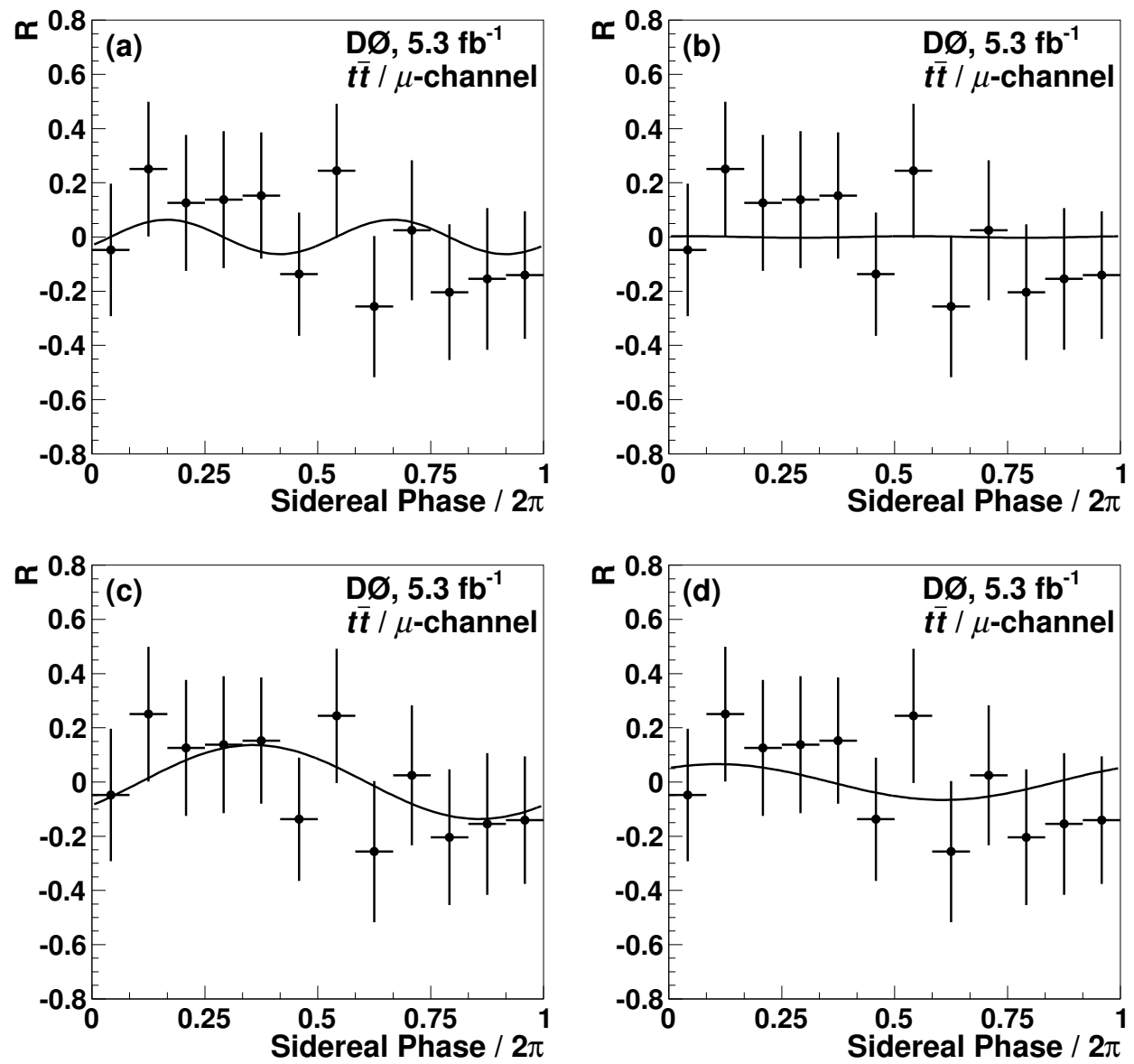

Figure F.8: Fitted $f_{\mathrm{SME}}\left(\phi_{i}\right)$ (line) and observed $R_{i}$ (crosses), $\mu+>3$-jets $t \bar{t}$ candidates, for $c_{\mu \nu}=0$ : (a) $d_{X X}$, (b) $d_{X Y}$, (c) $d_{X Z}$, (d) $d_{Y Z}$. 


\section{F.3 Simultaneous $e+>3$-jets and $\mu+>3$-jets}
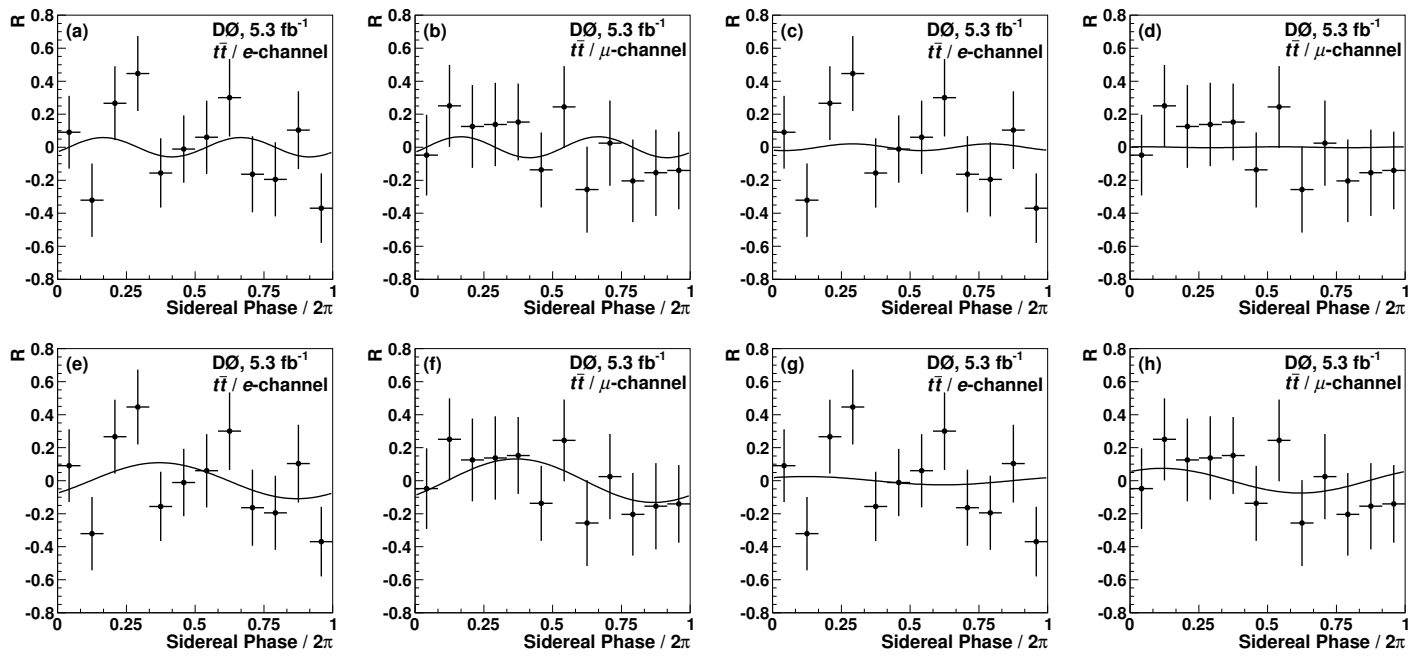

Figure F.9: Fitted $f_{\mathrm{SME}}\left(\phi_{i}\right)$ (line) and observed $R_{i}$ (crosses), $e+>3$-jets and $\mu+>3$ jets $t \bar{t}$ candidates, for $\left(c_{R}\right)_{\mu \nu}=0$ :

(a) $\left(c_{L}\right)_{X X}$ (e+jets), (b) $\left(c_{L}\right)_{X X}$ ( $\mu+$ jets), (c) $\left(c_{L}\right)_{X Y}(e+$ jets $),(d)\left(c_{L}\right)_{X Y}(\mu+$ jets $)$,

(e) $\left(c_{L}\right)_{X Z}$ (e+jets), (f) $\left(c_{L}\right)_{X Z}$ ( $\mu+$ jets), (g) $\left(c_{L}\right)_{Y Z}(e+$ jets $),(\mathrm{g})\left(c_{L}\right)_{Y Z}$ ( $\mu+$ jets $)$. 

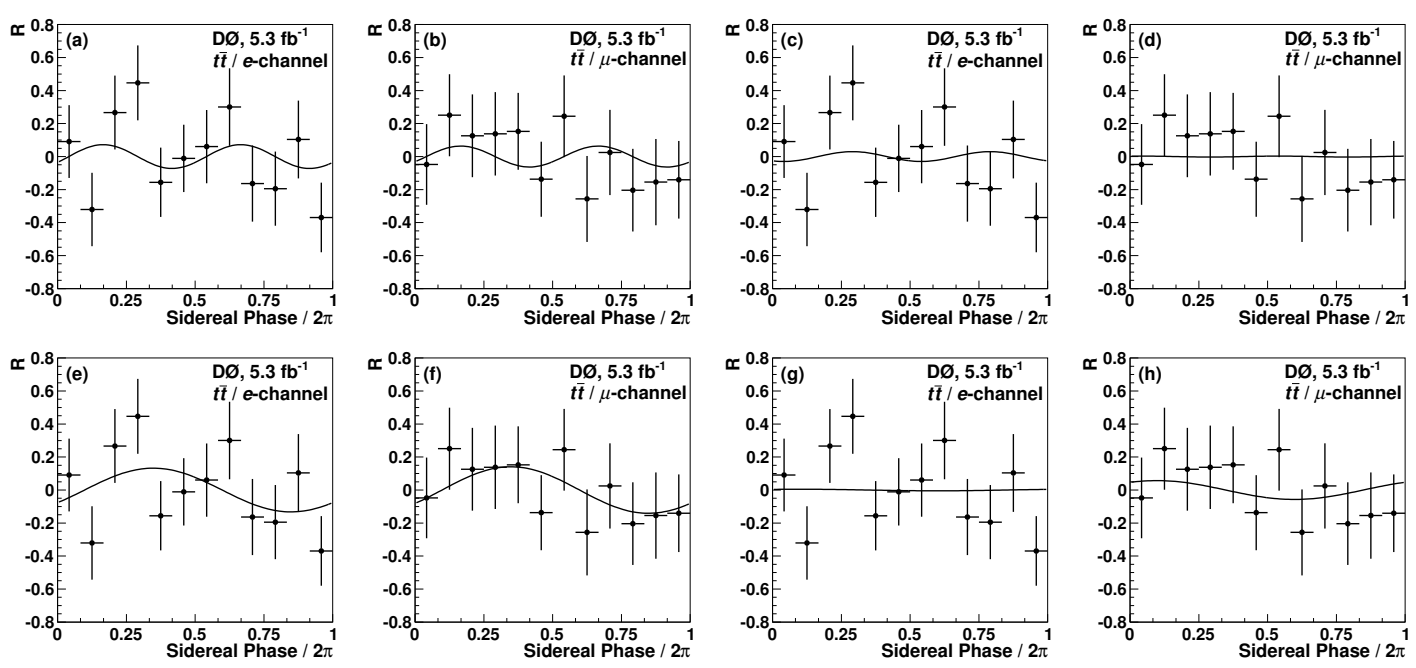

Figure F.10: Fitted $f_{\mathrm{SME}}\left(\phi_{i}\right)$ (line) and observed $R_{i}$ (crosses), $e+>3$-jets and $\mu+>3$ jets $t \bar{t}$ candidates, for $\left(c_{L}\right)_{\mu \nu}=0$ :

(a) $\left(c_{R}\right)_{X X}$ (e+jets), (b) $\left(c_{R}\right)_{X X}$ ( $\mu+$ jets), (c) $\left(c_{R}\right)_{X Y}(e+$ jets $),(d)\left(c_{R}\right)_{X Y}(\mu+$ jets $)$,

(e) $\left(c_{R}\right)_{X Z}(e+$ jets $),(f)\left(c_{R}\right)_{X Z}$ ( $\mu+$ jets $),(\mathrm{g})\left(c_{R}\right)_{Y Z}(e+\mathrm{jets}),(\mathrm{g})\left(c_{R}\right)_{Y Z}(\mu+\mathrm{jets})$. 

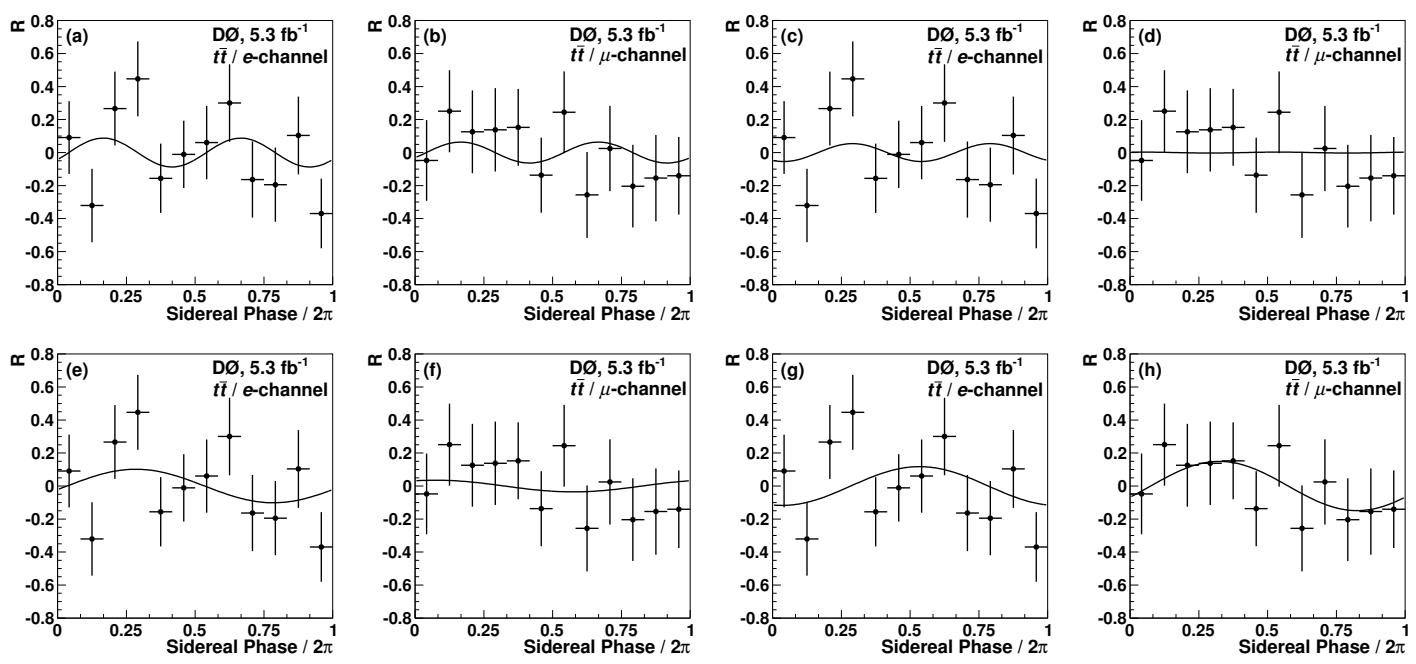

Figure F.11: Fitted $f_{\mathrm{SME}}\left(\phi_{i}\right)$ (line) and observed $R_{i}$ (crosses), $e+>3$-jets and $\mu+>3$ jets $t \bar{t}$ candidates, for $d_{\mu \nu}=0$ :

(a) $c_{X X}$ (e+jets), (b) $c_{X X}$ ( $\mu+$ jets), (c) $c_{X Y}$ (e+jets), (d) $c_{X Y}$ ( $\mu+$ jets), (e) $c_{X Z}$ (e+jets), (f) $c_{X Z}$ ( $\mu+$ jets), (g) $c_{Y Z}$ (e+jets), (g) $c_{Y Z}$ ( $\mu+$ jets). 

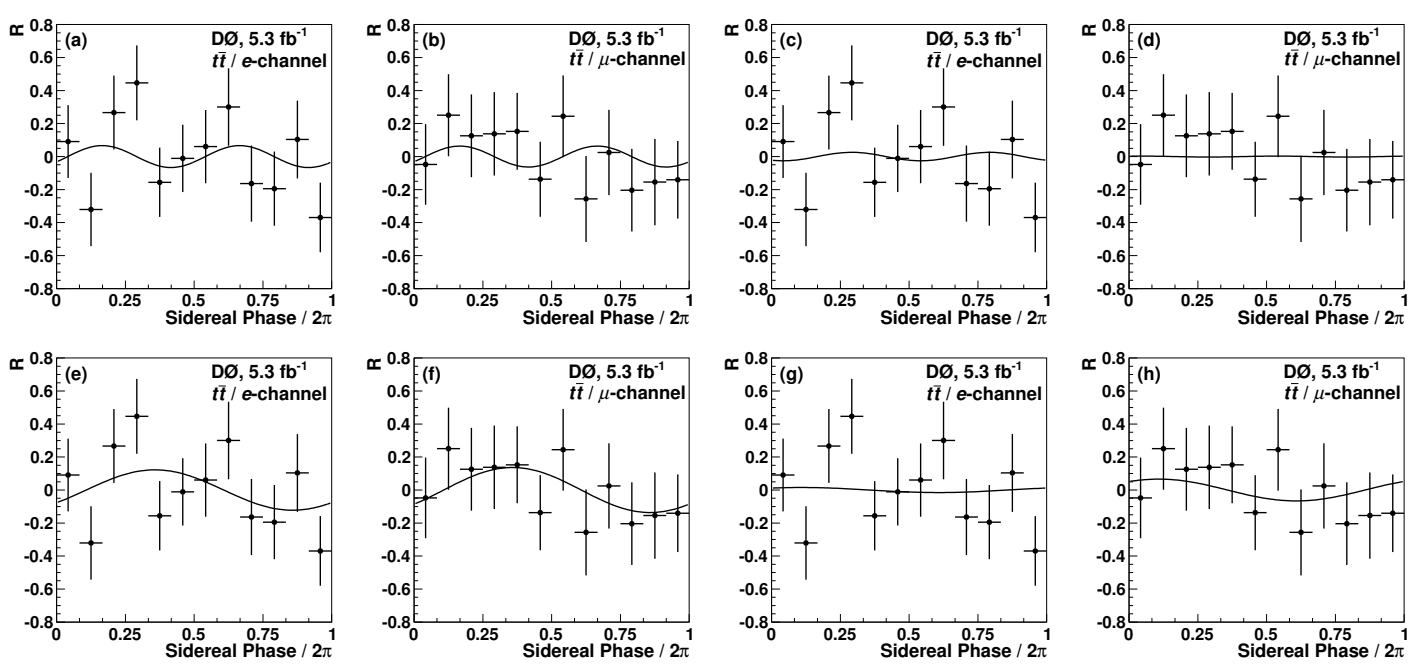

Figure F.12: Fitted $f_{\mathrm{SME}}\left(\phi_{i}\right)$ (line) and observed $R_{i}$ (crosses), $e+>3$-jets and $\mu+>3$ jets $t \bar{t}$ candidates, for $c_{\mu \nu}=0$ :

(a) $d_{X X}$ (e+jets), (b) $d_{X X}$ ( $\mu+$ jets), (c) $d_{X Y}$ (e+jets), (d) $d_{X Y}$ ( $\mu+$ jets), (e) $d_{X Z}$ (e+jets), (f) $d_{X Z}$ ( $\mu+$ jets), (g) $d_{Y Z}$ (e+jets), (g) $d_{Y Z}$ ( $\mu+$ jets). 


\section{Denver Whittington}

\section{Contact Information}

Physics Department

Indiana University

727 E. Third St.

Bloomington, IN 47405-7105
Office Phone: (812) 855-1056

Email: dwwhitti@indiana.edu

\section{Research Interests}

- Experimental High Energy Particle Physics

- Software Development for Event Simulation and Reconstruction

- Software Development for Particle Detector Monitoring

- Physics Education and Public Outreach

\section{Education}

2012 Ph.D., Physics (July 2012)

Indiana U.

Searches for Lorentz Violation in Top-Quark Production and Decay at

Hadron Colliders

Thesis Advisor: Professor Harold G. Evans

2005 M.S., Physics

2004 B.A., Physics \& Mathematics summa cum laude

Indiana $\mathrm{U}$. Anderson U.

\section{Awards}

2010 - $2011 \quad$ College of Arts and Sciences Dissertation Year Research Fellowship Indiana U.

2009 Best Scientific Content poster award, ATLAS Physics Workshop of the Americas ATLAS experiment 


\section{Research and Professional Experience}

$\begin{array}{lll}2010-\text { Present } & \begin{array}{l}\text { Graduate Student Collaborator } \\ \text { Graduate Student Member }\end{array} & \begin{array}{l}\text { D Experiment } \\ \text { Indiana University Center } \\ \text { for Spacetime Symmetries }\end{array} \\ 2006-\text { Present } & \begin{array}{l}\text { Graduate Student Member } \\ \text { ATLAS Experiment }\end{array} \\ 2008-2009 & \begin{array}{l}\text { Software Development and Commissioning Co- } \\ \text { coordinator, Transition Radiation Tracker }\end{array} & \text { ATLAS Experiment } \\ 2006-2010 & \text { TRT Conditions Software expert } & \text { ATLAS Experiment }\end{array}$

\section{Teaching Experience}

2011 - $2012 \quad$ Guest Lecturer, P114 Physics for Poets

2010

$2004-2006$

$2000-2004$
Undergraduate Research Supervisor (Ben Weinert)

Associate Instructor, Introductory Physics

Teaching Assistant, Physics and Astronomy
Indiana $\mathrm{U}$. Indiana U. Indiana U. Anderson U.

\section{Professional Service and Public Outreach}

2012

2011 - present

$2010-2011$

$2010-2011$

$2010-2011$

2010

$2009-2011$

$2009-2011$
AP Physics Instructor, Foundations in Science and Mathematics (high school summer program)

Tutor to undergraduates in physics

Physics \& Astronomy Open House Planning Committee

Physics Outreach Committee

Physics Undergraduate Curriculum Committee

Subject Matter Expert

Undergraduate Physics Club graduate student mentor Various elementary school science outreach events
Indiana $\mathrm{U}$.

Indiana U. Indiana U. Indiana U. Indiana $\mathrm{U}$.

Wisdom Tools,

Bloomington, IN Indiana $\mathrm{U}$. Indiana $\mathrm{U}$.

\section{Technical Skills}

- Data analysis with ROOT software

- Programming with $\mathrm{C}++$, Python, and FORTRAN

- Offline detector monitoring software development

- Software development in the Gaudi framework

- Data analysis in grid computing environments

- Linux and Windows operating systems

- Web page design with HTML, CSS, and PHP 


\section{Selected Publications}

\section{Primary Author}

- "Search for violation of Lorentz invariance in top quark pair production and decay," V.M. Abazov et al. (DØ Collaboration), Phys. Rev. Lett. 108, 261603 (2012)

- "Sensitivity to Lorentz Violation in the Top-Quark Sector at the LHC," D. Whittington, in Proceedings of the Fifth Meeting on CPT and Lorentz Symmetry, edited by V.A. Kostelecký (World Scientific, Hackensack, NJ, 2010)

\section{Internal Notes}

- "Studies of Lorentz invariance in $t \bar{t}$ production and decay," D0 Note 6281 (2012)

- "Measurement of $\beta \gamma$ for $R$-hadrons using the ATLAS TRT low-threshold bit pattern," ATL-COM-INDET-2010-154

- "Specific Energy Loss Estimators using the ATLAS TRT," ATL-COM-INDET-2010-150

- "Particle ID Discriminators Using Drift Times in the ATLAS TRT Barrel," ATL-COM-INDET-2010-079

- " $\Psi$ Variable for Muon Isolation : $Z \rightarrow \mu \mu, W \rightarrow \mu \nu$ and $t \rightarrow \mu \nu b$ in FDR2 Data," ATL-COM-PHYS-2009-049

- "High- $p_{T}$ Top Tagging in Muon + Jet Channel," ATL-COM-PHYS-2008-234

\section{Detector Publications}

- "The ATLAS Experiment at the CERN Large Hadron Collider," The ATLAS Collaboration, G Aad et al., 2008 JINST 3 S08003

- "The ATLAS Transition Radiation Tracker (TRT) proportional drift tube: design and performance,"

ATLAS TRT collaboration, E. Abat et al., 2008 JINST 3 P02013

- "The ATLAS TRT Barrel Detector,"

The ATLAS TRT collaboration, E Abat et al., 2008 JINST 3 P02014

- "The ATLAS TRT end-cap detectors,"

The ATLAS TRT collaboration, E Abat et al, 2008 JINST 3 P10003

- "The ATLAS TRT electronics,"

The ATLAS TRT collaboration, E Abat et al, 2008 JINST 3 P06007 


\section{Conference Presentations}

2012 Search for Violation of Lorentz Invariance in $t \bar{t}$ Production and Decay at the DØ Experiment APS April Conference

2012 Search for Lorentz Invariance Violation in Top-Quark Production and Decay at DØ All DØ Meeting

2012 Search for Lorentz Invariance Violation in Top-Quark Production and Decay at DØ DØ Winter Physics Workshop

2011 Update on Lorentz Invariance Violation in Top-Quark Production and Decay at DØ General Top Meeting of the DØ Collaboration Week

2011 Searching for Lorentz Invariance Violation in Top-Quark Production and Decay at DØ DØ Summer Workshop in Princeton

2010 Particle Physics at ATLAS: Exploring the Energy Frontier Advanced College Project Review Seminar (Physics)

2010 Sensitivity to Lorentz violation in the top quark sector at the ATLAS detector (Poster) Fifth Meeting on CPT and Lorentz Symmetry

2010 Describing the TRT Residual Distribution ATLAS ID Week (June)

2010 Describing the TRT Residual (and Resolution) ATLAS ID Week (March)

2009 Reconstructing boosted top decays - a window on new physics at ATLAS (Poster) ATLAS Physics Workshop of the Americas

2009 TRT Conditions Data for Offline TRT Offline Monitoring Working Workshop

2008 Search for Exotics with Top Quark Final States North/South America ATLAS Top Meeting 